\title{
Delay Extraction Based Macromodeling Techniques for Tabulated/Measured Data
}

\author{
by \\ Andrew Charest, B. Eng.

\begin{abstract}
A thesis submitted to the
Faculty of Graduate Studies and Research

in partial fulfillment of the requirements for the degree of Master of Applied Science
\end{abstract}
Ottawa-Carleton Institute for Electrical Engineering
Department of Electronics
Faculty of Engineering
Carleton University
Ottawa, Canada

September 2007

(c) Andrew Charest, 2007 


$\begin{array}{ll}\begin{array}{l}\text { Library and } \\ \text { Archives Canada }\end{array} & \begin{array}{l}\text { Bibliothèque et } \\ \text { Archives Canada }\end{array} \\ \begin{array}{l}\text { Published Heritage } \\ \text { Branch }\end{array} & \begin{array}{l}\text { Direction du } \\ \text { Patrimoine de l'édition }\end{array} \\ \begin{array}{l}\text { 395 Wellington Street } \\ \text { Ottawa ON K1A ON4 }\end{array} & \begin{array}{l}\text { 395, rue Wellington } \\ \text { Ottawa ON K1A ON4 } \\ \text { Canada }\end{array}\end{array}$

Your file Votre référence ISBN: 978-0-494-33642-7 Our file Notre référence ISBN: 978-0-494-33642-7

NOTICE:

The author has granted a nonexclusive license allowing Library and Archives Canada to reproduce, publish, archive, preserve, conserve, communicate to the public by telecommunication or on the Internet, loan, distribute and sell theses worldwide, for commercial or noncommercial purposes, in microform, paper, electronic and/or any other formats.

The author retains copyright ownership and moral rights in this thesis. Neither the thesis nor substantial extracts from it may be printed or otherwise reproduced without the author's permission.
AVIS:

L'auteur a accordé une licence non exclusive permettant à la Bibliothèque et Archives Canada de reproduire, publier, archiver, sauvegarder, conserver, transmettre au public par télécommunication ou par l'Internet, prêter, distribuer et vendre des thèses partout dans le monde, à des fins commerciales ou autres, sur support microforme, papier, électronique et/ou autres formats.

L'auteur conserve la propriété du droit d'auteur et des droits moraux qui protège cette thèse. $\mathrm{Ni}$ la thèse ni des extraits substantiels de celle-ci ne doivent être imprimés ou autrement reproduits sans son autorisation.
In compliance with the Canadian

Privacy Act some supporting forms may have been removed from this thesis.

While these forms may be included in the document page count, their removal does not represent any loss of content from the thesis.
Conformément à la loi canadienne sur la protection de la vie privée, quelques formulaires secondaires ont été enlevés de cette thèse.

Bien que ces formulaires aient inclus dans la pagination, il n'y aura aucun contenu manquant.

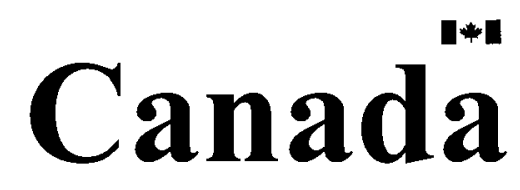




\section{Abstract}

Due to increasing operating frequencies and circuit densities, accurate and efficient signal integrity analysis has become a major limitation for high-speed VLSI design. Signal propagation effects such as delay, distortion, reflections, and crosstalk can seriously limit the overall performance of high-speed systems. Accounting for these effects in analytical device models is not always possible and subsequently, high-speed modules are characterized by tabulated parameters $(\mathrm{Y}, \mathrm{Z}, \mathrm{S}$, or $\mathrm{H})$, obtained either through measurements or rigorous full-wave electromagnetic simulations.

Prominent approaches for transient analysis of subnetworks characterized by tabulated data are based on approximating the data with rational functions and subsequently, synthesizing a SPICE compatible macromodel. However, for networks with long delays, these macromodels require high-order approximations.

In this thesis, a novel algorithm is developed to produce a compact macromodel through delayed rational function approximations. The proposed macromodel provides a transient speedup of $10-50$ times over conventional macromodels. 


\section{Acknowledgments}

I would like to especially express my deepest gratitude to Prof. Michel Nakhla and Prof. Ram Achar for providing motivation when I needed most, their expert insight, valuable guidance and unparalleled patience. Their friendly manner and wise judgment made this thesis an enjoyable and refreshing experience.

Special thanks are extended to Dr. Dharmendra Saraswat, Dr. Nick Soveiko, Prof. Pavan Gunupudi, and Prof. Tom Smy for the valuable discussions, helpful comments, ideas, and suggestions.

I would like to thank my colleagues A. Sridhar, D. Paul, N. Nakhla, L. Filipovic, A. Jerome, C. Chen, B. Nouri, and V.Ambalavanar for all their help and support.

I leave a special note of appreciation to Diana Ioan and my family for their love and many years of unconditional support and encouragement. 
Dedicated to my parents,

Elizabeth Paveck and

Robert Charest,

for showering unconditional love,

boundless patience, and supreme guidance.

iv 


\section{Contents}

1 Introduction 1

1.1 Motivation ....................... 1

1.2 Contributions ........................... 5

1.3 Organization of the Thesis . . . . . . . . . . . 7

2 Interconnect Macromodeling and Long-Delay Networks $\quad 8$

2.1 Introduction . . . . . . . . . . . . . . . 8

2.2 Interconnect Macromodeling . . . . . . . . . . . . . . 9 9

2.2 .1 Problem Formulation . . . . . . . . . . . . . 10

2.2.2 Matrix Rational Approximations (MRA) . . . . . . . . . 12

2.3 Macromodeling Issues . . . . . . . . . . . . . . . . 13

2.3 .1 Passivity . . . . . . . . . . . . . . . 15

2.3 .2 Causality ........................... 16

2.3.3 Long Delay Networks . . . . . . . . . . . . . . 17

2.4 DEPACT Macromodeling Algorithm . . . . . . . . . . . 18

2.4.1 Frequency Dependent Parameters . . . . . . . . . . . 19

2.4.2 Estimation of the Order of Approximation . . . . . . . 20

V 
2.5 Macromodeling Algorithms for Interconnects Characterized by Tabulated Data . . . . . . . . . . . . . . . . . . . 20

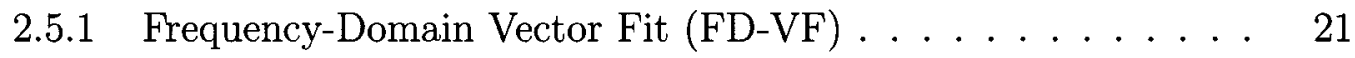

2.5.2 Time-Domain Vector Fit (TD-VF) $\ldots \ldots \ldots \ldots$

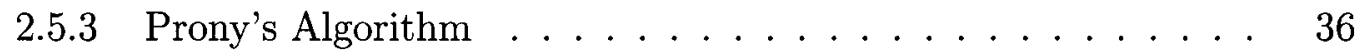

2.6 Macromodeling Techniques for Long-Delay

Networks . . . . . . . . . . . . . . . . . . . 38

2.6.1 Delay based Macromodeling via Hilbert Transforms . . . . . . 39

2.6.2 Delay based Vector Fitting using Optimization . . . . . . . . 41

2.6.3 Delay based Macromodeling via Gabor Transforms . . . . . . 44

3 Passive Macromodeling of Transmission Line Type Interconnects $\begin{array}{ll}\text { Characterized by Tabulated Data } & 47\end{array}$

3.1 Introduction . . . . . . . . . . . . . . . . . 47

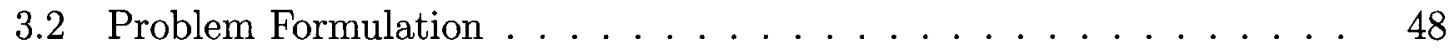

3.3 A Unique Matrix Logarithm . . . . . . . . . . . . . . . . . . . 49

3.4 Formulation of Hybrid Model . . . . . . . . . . . . . . . 51

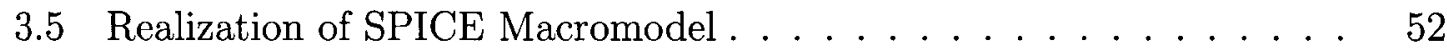

3.6 Numerical Results . . . . . . . . . . . . . . . . . . . . . 57

3.6.1 Example 1: Two-Port Transmission Line . . . . . . . . 57

3.6.2 Example 2: Four-Port Transmission Line . . . . . . . . . 60

3.7 Summary . . . . . . . . . . . . . . . . . 63

4 Macromodeling via Delayed

Rational Functions Using Prony's Algorithm 64

vi 
4.1 Introduction . . . . . . . . . . . . . . . . . . 64

4.2 Development of the Proposed Algorithm . . . . . . . . . . . 65

4.3 Determination of Partition Points . . . . . . . . . . . . . 67

4.4 Continuity Enforcement _ . . . . . . . . . . . . . . . . . . . 69

4.5 Numerical Results . . . . . . . . . . . . . . . . . . . . 70

$4.5 .1 \quad$ Example $1 \ldots \ldots \ldots \ldots \ldots \ldots$

$4.5 .2 \quad$ Example $2 \ldots \ldots \ldots \ldots \ldots \ldots \ldots \ldots$

$4.5 .3 \quad$ Example $3 \ldots \ldots \ldots \ldots \ldots \ldots$

4.6 Summary . . . . . . . . . . . . . . . . 83

5 Delay Extraction based Compact Interconnect Macromodeling Algorithm for Long Delay Networks $\quad 84$

5.1 Introduction . . . . . . . . . . . . . . . . . . . 84

5.2 Preliminaries and the Underlying Principle of the Proposed Algorithm 85

5.3 Formulation of Delayed Rational Functions . . . . . . . . . . . . 89

5.4 Continuity Enforcement $\ldots \ldots \ldots \ldots \ldots$

5.5 Determination of Partition Points . . . . . . . . . . . . . 99

5.6 Numerical Results . . . . . . . . . . . . . . . . . . . . . . . . 101

$5.6 .1 \quad$ Example $1 \ldots \ldots \ldots \ldots \ldots \ldots$

5.6 .2 Example $2 \ldots \ldots \ldots \ldots \ldots \ldots$

$5.6 .3 \quad$ Example $3 \ldots \ldots \ldots \ldots \ldots \ldots$

5.7 Summary . . . . . . . . . . . . . . . . . . . . 114

6 Realization of Equivalent Subcircuits from Delayed Rational Functions $\quad 115$

vii 


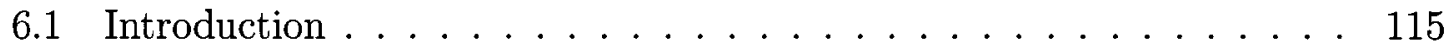

6.2 Macromodel Synthesis _ . . . . . . . . . . . . . . . . 116

6.2.1 Example: Macromodel Synthesis _ . . . . . . . . . . 117

6.3 Inclusion of Complex Poles . . . . . . . . . . . . . . . . . . . . 119

6.3.1 Example: Complex Pole Modifications . . . . . . . . . . 120

6.4 Construction of Equivalent Subcircuits _ . . . . . . . . . . . 122

6.5 Circuit Interface for Scattering Parameter based Macromodels . . . . . . . . . . . . . . . . . . . . . . 124

6.5.1 Example: Interface for Scattering Parameter based Macromodels . . . . . . . . . . . . . . . . 126

6.6 Summary . . . . . . . . . . . . . . . . . . . . 128

7 Conclusions and Future Work $\quad 129$

7.1 Summary . . . . . . . . . . . . . . . . . . . 129

7.2 Future Work . . . . . . . . . . . . . . . . . 131

A Wavelets 132

A.1 Orthogonal Refinement . . . . . . . . . . . . . . . 133

A.2 Refinement Relations . . . . . . . . . . . . . . . . . 134

A.3 Fast Wavelet Transform $\ldots \ldots \ldots \ldots \ldots$

A.4 Boundary Wavelets . . . . . . . . . . . . . . 138

viii 


\section{List of Figures}

1.1 High-speed interconnect effects. . . . . . . . . . . . 2

1.2 Sample response data for long delay line. . . . . . . . . . . . . . . 4

2.1 Multiconductor transmission line system. . . . . . . . . . 11

2.2 Transient response of a stable, but non-passive macromodel with passive terminations. . . . . . . . . . . . . . . 14

2.3 Illustration of significance of passivity: a) Stable-non-passive macromodel b) Unstable macromodel with passive loads. . . . . . . . . . 16

3.1 A comparison of the matrix logarithm computation . . . . . . 50

3.2 Macromodel realization of product terms in (3.8) . . . . . . . . 53

3.3 Equivalent circuit representation for the realization of lossless coupled

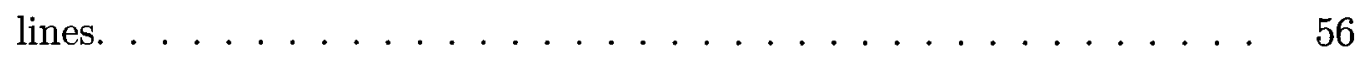

3.4 Circuit for example $1 . \ldots \ldots \ldots \ldots$. . . . . . . . . 57

3.5 Frequency-domain comparisons for example 1. . . . . . . . . . 58

3.6 Comparison of transient response for example $1 \ldots \ldots$. . . . . . 59

3.7 Circuit for example $2 . \ldots \ldots \ldots$. . . . . . . . . . 60

3.8 Frequency-domain comparisons for example 2. . . . . . . . . . 61 
3.9 Comparison of transient response for example $2 \ldots \ldots$. . . . . . . 62

4.1 Segmentation of the time-domain waveform into $Q$ intervals. . . . . . 66

4.2 Circuit for example $1 . \ldots \ldots \ldots 72$

4.3 Time-frequency analysis of the response data. . . . . . . . . 73

4.4 Frequency-domain comparisons for example 1. . . . . . . . . 74

4.5 Transient comparison for example 1. . . . . . . . . . . 75

4.6 Circuit for example $2 \ldots \ldots \ldots \ldots 76$

4.7 Time-frequency analysis for example $2 \ldots \ldots \ldots$. . . . . . . 77

4.8 Frequency-domain comparisons for example 2. . . . . . . . 78

4.9 Transient comparison for example 2. . . . . . . . . . . . 79

4.10 Circuit for example $3 . \ldots \ldots \ldots$. . . . . . . . . 80

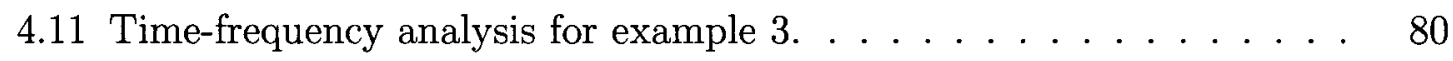

4.12 Frequency-domain comparisons for example 3. . . . . . . . . . . 81

4.13 Comparison of transient responses for example $3 . \ldots$. . . . . . . 82

5.1 Transient response for a lossy transmission line. . . . . . . . . . . 87

5.2 Frequency response of the transfer function for interval $\left[\tau_{s_{0}}, \tau_{s_{1}}\right]$ of Fig.

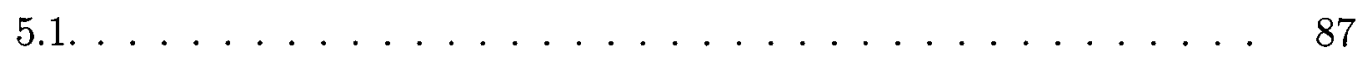

5.3 Frequency response of the transfer function for interval $\left[\tau_{s_{0}}, \tau_{s_{2}}\right]$ of Fig.

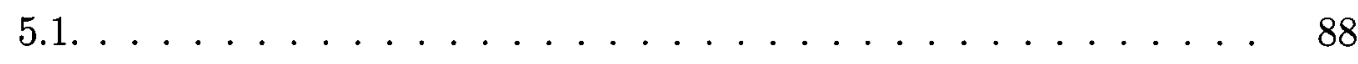

5.4 Illustrative graphs showing time-domain data and partition points for fitting of first segment. . . . . . . . . . . . . . . 92

5.5 Illustrative graphs showing time-domain data and partition points for fitting of second segment. . . . . . . . . . . . . 94 
5.6 Circuit for example 1. . . . . . . . . . . . . . . 102

5.7 Time-frequency analysis of the response data. . . . . . . . . . 103

5.8 Frequency-domain comparisons for example 1. . . . . . . . . . . 104

5.9 Transient comparison for example 1. . . . . . . . . . . . . 105

5.10 Expanded view of the transient response in Fig. 5.9. . . . . . . . 105

5.11 Circuit for example $2 . \ldots \ldots \ldots \ldots \ldots$

5.12 Time-frequency analysis of the response data for example $2 . \quad \ldots \quad 107$

5.13 Frequency-domain comparisons for example 2. . . . . . . . . . 108

5.14 Transient comparison for example $2 . \ldots \ldots \ldots$

5.15 Circuit for example $3 . \ldots \ldots \ldots \ldots \ldots$

5.16 Time-frequency analysis for example $3 \ldots \ldots \ldots \ldots$

5.17 Frequency-domain comparisons for example $3 \ldots \ldots \ldots \ldots$

5.18 Comparison of transient responses for example $3 . \ldots \ldots \ldots \ldots$

6.1 Multiport linear subnetwork $\pi \ldots \ldots \ldots \ldots \ldots$

6.2 Equivalent subcircuits for admittance parameters represented by DDEs

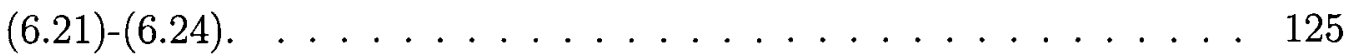

6.3 Equivalent subcircuits for scattering parameters. . . . . . . . . 127

A.1 Refinement in the basis of pulse functions. . . . . . . . . . 133

A.2 Refinement of the Haar basis using the orthogonal complement. . . . 134

A.3 Refinement relations for the Haar basis. . . . . . . . . . . . 135

A.4 Scaling function for orthogonal Daubechies wavelet with four filter coefficients. . . . . . . . . . . . . . . . . . . 136 
A.5 Computing one level of FWT with FIR filter bank $(x[k]$ represents a vector of length $k) \ldots \ldots \ldots \ldots \ldots \ldots \ldots$

A.6 Wavelet decomposition tree $\left(\tilde{d}_{i}\right.$ represents the vector of detail coefficients from each transform) . . . . . . . . . . . . . . 139 


\section{List of Tables}

3.1 A summary of the CPU performance of the macromodels . . . . . . 57

4.1 A summary of the CPU performance of the macromodels . . . . . 71

5.1 A summary of the CPU performance of the macromodels. . . . . . . 101

xiii 


\section{List of Acronyms}

VLSI Very Large Scale Integration

CAD Computer-Aided Design

MNA Modified Nodal Analysis

CPU Central Processing Unit

p.u.l per-unit-length

DEPACT Delay Extraction-Based Passive Compact Transmission-Line

SPICE Simulation Program with Integrated Circuit Emphasis

w.r.t with respect to

FFT Fast Fourier Transform

IFFT Inverse Fast Fourier Transform

FWT Fast Wavelet Transform

RHP Right Hand Plane

LHP Left Hand Plane

MoC Method of Characteristics

MTL Multiconductor Transmission Line

xiv 


$\begin{array}{ll}\text { ODE } & \text { Ordinary Differential Equation } \\ \text { DDE } & \text { Delayed Differential Equation } \\ \text { MRA } & \text { Matrix Rational Approximation } \\ \text { FD-VF } & \text { Frequency-Domain Vector Fit } \\ \text { TD-VF } & \text { Time-Domain Vector Fit } \\ \mathrm{H} & \text { Hybrid (Parameters) } \\ \mathrm{Y} & \text { Admittance (Parameters) } \\ \mathrm{Z} & \text { Impedance (Parameters) } \\ \mathrm{S} & \text { Scattering (Parameters) }\end{array}$




\section{List of Symbols}

A

$\mathbf{A}^{T}$

A*

0

I

$\Re^{N}$

$\Re^{N \times N}$

$\omega$

$\mathfrak{L}$

$s$

$\arg (\phi)$

$\tilde{a}_{i}$

$\tilde{b}_{i}$

$j$

$\mathfrak{R e}(x)$

$\mathfrak{I m}(x)$
Matrix A

Matrix A transposed

Matrix A conjugate

Matrix of zeros

Identity matrix

The set of all real vectors of size $N$

The set of all real matrices of size $N \times N$

Angular frequency

Laplace operator

Independent variable of the Laplace-domain $(=j \omega)$

Argument or phase angle of the complex value $\phi$

$i^{\text {th }}$ incident scattering wave in the time-domain

$i^{\text {th }}$ reflected scattering wave in the time-domain

$\sqrt{-1}$

Real part of the complex number $x$

Imaginary part of the complex number $x$

xvi 


\section{Chapter 1}

\section{Introduction}

\subsection{Motivation}

The ever increasing design constraints of submicron VLSI chips are placing enormous demands on computer-aided design (CAD) tools to perform accurate signal integrity analysis of high-speed modules. At high frequencies, effects (shown in Fig. 1.1) such as reflections, crosstalk, ringing, signal distortion, and signal delay cause signal degradation and faulty system performance [1]-[5]. These effects have recently been highlighted by the increased integration of analog circuits with digital blocks, the demand for lower power consumption, and the miniaturization of designs. If not predicted at the early stages of design, high-speed circuit effects can severely degrade the system performance and, in many cases, render the circuit inoperable. Therefore, it is important to model and simulate high-speed interconnects as accurately and efficiently as possible [6]-[17].

There are two major issues that arise during the transient simulation of inter- 


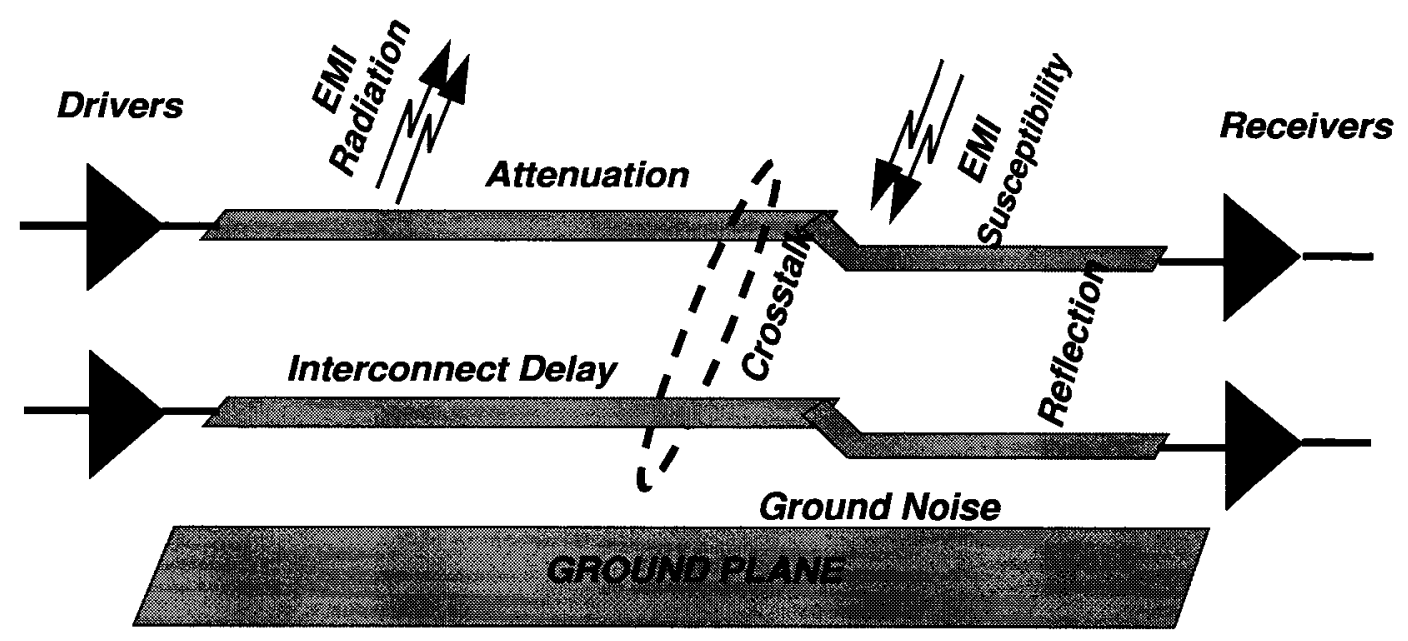

Fig. 1.1: High-speed interconnect effects.

connects. First, interconnect structures can consist of thousands of resistors, capacitors, inductors, transmission lines, and other high-frequency devices that make transient simulation prohibitively CPU expensive. Second, interconnect terminations are generally nonlinear devices, such as drivers and receivers, which are modeled in the time-domain, while interconnects structures are generally best described in the frequency-domain. This type of mixed/frequency time problem cannot always be handled by ordinary differential equation (ODE) solvers such as SPICE. As a result, a significant amount of research has been directed at solving mixed frequency/time problems [3]-[48].

There are several topics in the CAD of VLSI chips plagued by the mixed frequency/time problem. One such topic, is the modeling and characterization of high-speed interconnects, packages, vias, connectors, nonuniform transmission lines, and on-chip passive components, such as inductors and transformers, by tabulated data [18]-[42]. This data is obtained through either measurements or rigorous full-wave electromag- 
netic simulations. It can be in the form of scattering (S), admittance (Y), impedance $(\mathrm{Z})$, or hybrid $(\mathrm{H})$ parameters as a function of frequency. The data may also be given in the time-domain, as a set of response data, $\{y(t)\}$, due to an excitation, $\{x(t)\}$. The integration of this data with nonlinear elements in SPICE simulators is not always straightforward, and even state of the art simulators do not adequately handle this data [49]-[52]. As a result, several publications regarding the integration and simulation of tabulated data in SPICE-like simulators have been developed.

Most of these publications develop rational function based approximation algorithms, which are converted into a set of equivalent subcircuits for SPICE integration [3]. Initially, the macromodels produced from these methods were not guaranteed to be passive. Passivity is important because, stable, but non-passive macromodels connected to other passive networks can yield unstable responses [44]. However, recent methods have been developed to both check, and enforce passivity in macromodels constructed from rational approximations [38]-[40].

Attempts to improve these fitting algorithms have only increased the accuracy and convergence of the fitting algorithms themselves, but have done little to reduce the size, number of poles, and efficiency of the resulting macromodels in transient simulators [31]-[35]. In addition, higher operating frequencies and longer system delays are resulting in extremely high-order rational approximations and subsequently, prohibitively large macromodels for SPICE simulation.

In order to illustrate the difficulties associated with long delay lines, consider the time-domain response of an electrically long interconnect in Fig. 1.2(a). The corresponding frequency-domain response is shown in Fig. 1.2(b). When this response is approximated (up to $8 \mathrm{GHz}$ ) using direct rational approximation [30], the number of 
poles required is very high (700 poles). This is mainly because the waveform under consideration consists of a long delay. Consequently, the size of the macromodel is very high (typically given by the product of the number of ports and poles), leading to slow transient analysis using SPICE-like simulators.

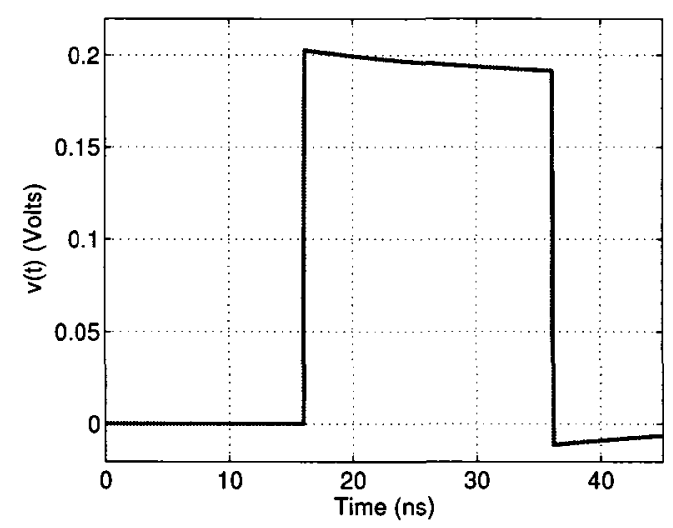

(a) Sample time-domain response.

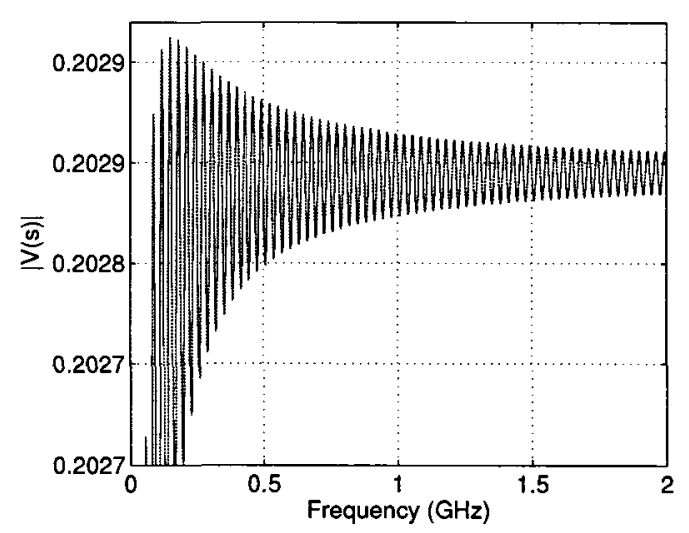

(b) Magnitude of the frequency-domain response.

Fig. 1.2: Sample response data for long delay line.

In order to overcome this problem for the case of transmission lines characterized by RLCG per-unit-length (p.u.l) parameters [10]--[13], the delay extraction-based passive compact transmission line (DEPACT) macromodeling algorithm was developed [7]-[9]. This algorithm uses a modified Lie formula (MLF) to approximate the exponential transmission line stamp as a product of lossless and lossy terms. The lossless terms contain the delay of the line and are efficiently simulated using the method of characteristics (MoC) [14]-[17], while the remaining lossy terms required a low-order MRA formulation to fit. The result is a significantly smaller and more efficient macromodel in the form of a set of delayed differential equations (DDEs), which could also be solved in standard SPICE simulators.

For the case of networks characterized by tabulated S, Y, Z, or H frequency- 
domain data, [23]-[26] present Hilbert transform based delay estimation. The work in [27], [28] incorporates delay estimation into the vector fitting algorithm using optimization. However, the methods in [23]-[28] extract only one dominant delay and disregard higher order reflections. These techniques may also fail if more than one delay is present in the response. In [29], a Gabor transform based macromodeling technique was developed for networks characterized by tabulated frequency-domain data. However, it still remains a challenging task to macromodel general high-speed circuits containing long delays and characterized by tabulated port responses.

The goal of this thesis is to develop new algorithms for generating compact, efficient, and accurate macromodels for high-speed networks characterized by tabulated data. Specific contributions of this thesis are given in the next section.

\subsection{Contributions}

The subject of this thesis is the macromodeling of tabulated data via delay extraction. The algorithms presented here provide an accurate and efficient approach for developing DDEs, which can be realized in SPICE simulators. The main contributions of this thesis are as follows.

1. A new algorithm is presented for the construction of a delay extraction-based macromodel for a single set of transmission lines from tabulated $\mathrm{H}, \mathrm{Y}, \mathrm{S}$, or $\mathrm{Z}$ parameters. The algorithm determines a unique logarithm for the exponential stamp of the $\mathrm{H}$ parameters. The delay extraction-based passive compact transmission-line macromodeling (DEPACT) algorithm is then applied to construct a compact macromodel for SPICE simulation [45]. 
2. A new algorithm is developed for compact macromodeling of high-speed circuits with long delays, characterized by tabulated data representing the impulse response. The algorithm is based on partitioning the time-domain impulse response and subsequently, approximating each partition (using Prony's algorithm) with a low-order sum of exponentials, delayed in time. This results in a compact low-order macromodel in the form of DDEs, which can be efficiently analyzed using SPICE-like simulators [46].

3. An improved algorithm is developed for the construction of delayed rational functions from tabulated time-domain data. This algorithm is applied to the time-domain multiport response data, $\{y(t)\}$, from an input excitation, $\{x(t)\}$. The multiport response data is partitioned and subsequently, each partition is approximated with a delayed rational function (in the frequency-domain) using the time-domain vector fit algorithm. The resulting compact low-order macromodel can be analyzed in SPICE-like simulators [47].

4. An efficient macromodel is developed for interfacing delayed rational functions with standard SPICE-like simulators [47].

The proposed macromodels produce several advantages over conventional techniques. They

a) generate low-order macromodels, thereby leading to faster SPICE based transient simulations,

b) can handle data with multiple delays, 
c) overcome the ringing associated with the high-order macromodels by virtue of delay extraction and low-order macromodeling.

\subsection{Organization of the Thesis}

This thesis is organized as follows. Chapter 2 reviews current macromodeling techniques for both interconnects, and networks characterized by tabulated data. Chapter 3 presents a new macromodeling algorithm for the transmission line type interconnects characterized by tabulated data. The algorithm extends the DEPACT macromodeling algorithm to handle tabulated S, Y, Z, or H parameters. Chapter 4 presents a new algorithm, which constructs a delayed rational function for general structures characterized by tabulated data. The algorithm partitions the data at high-frequency points localized in the time-domain using wavelet analysis and subsequently, fits each of the resulting partitions using Prony's algorithm. Numerical examples, which validate the efficiency of the proposed algorithm, are also presented in chapter 4 . In chapter 5, a novel algorithm is developed for the construction of delayed rational functions from tabulated data. This algorithm uses time-domain vector fit to approximate each of the segments, partitioned using modified wavelet analysis techniques developed in chapter 4 . Chapter 5 concludes with numerical examples, which validate the efficiency of the newly proposed algorithm. Next, chapter 7 develops a SPICE compatible model for networks described by delayed rational functions. Chapter 7 presents conclusions and proposed future research. 


\section{Chapter 2}

\section{Interconnect Macromodeling and Long-Delay Networks}

\section{$2.1 \quad$ Introduction}

As discussed in chapter 1, increasingly complex devices, combined with higher operating frequencies are making it difficult to obtain analytical models of high-speed modules for the purpose of transient simulation. As a result, networks are characterized by tabulated data, given in either the time-domain or frequency-domain. The simulation of this data with nonlinear components has been done traditionally by fitting the data with rational functions and subsequently, converting these functions into equivalent subcircuits for SPICE simulators [30], [36]--[40]. These rational function based macromodeling techniques are very effective, provided the order of the rational approximation is low. For high-order approximations, the transient simulation of the resulting (large) macromodel becomes prohibitively CPU expensive. 
In order to overcome this problem for transmission lines characterized by p.u.l parameters [10]-[13], delay extraction techniques were developed [7]-[9]. The resulting low-order (compact) macromodels, defined by DDEs, provide significant speed-up over conventional rational function based macromodels in SPICE-like simulators. For networks characterized by tabulated $\mathrm{S}, \mathrm{Y}, \mathrm{Z}$, or $\mathrm{H}$ parameters, several techniques have been introduced [23]-[29]. However, it still remains a challenging task to effectively produce delay-extraction based macromodels for networks characterized by tabulated port response data.

In this chapter, a brief overview of conventional macromodeling techniques for networks characterized by tabulated data p.u.l parameters is given, along with macromodeling techniques developed for long delay interconnects characterized by tabulated port response data. This chapter is organized as follows. Section 2.2 reviews interconnect macromodeling. Section 2.3 briefly describes key macromodeling issues and is followed by section 2.4, which outlines the DEPACT algorithm. Next, section 2.5 describes rational function based fitting algorithms for tabulated data. Specifically, the popular frequency-domain vector fit (FD-VF) algorithm, the time-domain vector fit (TD-VF) algorithm, and Prony's algorithm. Section 2.6 concludes with a discussion of current macromodeling techniques for long-delay networks characterized by tabulated data.

\subsection{Interconnect Macromodeling}

As discussed in chapter 1, simulating distributed interconnects (best represented in the frequency-domain) with nonlinear devices (best represented in the time-domain) 
has been a topic of intense research over the past several years. Consequently, several publications relating to this problem have been presented [1], [7]-[17]. These include lumped segmentation [1], the method of characteristics (MoC) [14]-[17], matrix rational approximation (MRA) based algorithms [10]-[13], and the delay extraction-based passive compact transmission line (DEPACT) macromodeling algorithm [7]-[9], which combines the benefits of delay extraction from $\mathrm{MoC}$ and conservation of passivity from MRA. A review of the passive MRA and DEPACT methodologies is given below.

\subsubsection{Problem Formulation}

Consider the multiconductor transmission line (MTL) system shown in Fig. 2.1 with $N$ coupled conductors. This system can be modeled using Telegrapher's equations [1]

$$
\begin{aligned}
\frac{\partial}{\partial x} \boldsymbol{v}(x, t) & =-\mathbf{R} \boldsymbol{i}(x, t)-\mathbf{L} \frac{\partial}{\partial t} \boldsymbol{i}(x, t) \\
\frac{\partial}{\partial x} \boldsymbol{i}(x, t) & =-\mathbf{G} \boldsymbol{v}(x, t)-\mathbf{C} \frac{\partial}{\partial t} \boldsymbol{v}(x, t)
\end{aligned}
$$

where $\mathbf{R} \in \mathbb{R}^{N \times N}, \mathbf{L} \in \mathbb{R}^{N \times N}, \mathbf{C} \in \mathbb{R}^{N \times N}$, and $\mathbf{G} \in \mathbb{R}^{N \times N}$ are the per-unit-length (p.u.l) parameters. $\boldsymbol{v}(x, t) \in \mathbb{R}^{N}$ and $i(x, t) \in \mathbb{R}^{N}$ represent the voltage and current, respectively, as a function of position, $x$, and time, $t$. Equation (2.1) can be transformed into the Laplace-domain as an exponential matrix function of the form

$$
\left[\begin{array}{c}
\mathbf{V}(d, s) \\
\mathbf{I}(d, s)
\end{array}\right]=e^{\mathbf{Z}}\left[\begin{array}{c}
\mathbf{V}(0, s) \\
\mathbf{I}(0, s)
\end{array}\right]
$$


where

$$
\mathbf{Z}=(\mathbf{D}+s \mathbf{E}) d, \quad \mathbf{D}=\left[\begin{array}{cc}
\mathbf{0} & -\mathbf{R} \\
-\mathbf{G} & \mathbf{0}
\end{array}\right], \quad \mathbf{E}=\left[\begin{array}{cc}
\mathbf{0} & -\mathbf{L} \\
-\mathbf{C} & \mathbf{0}
\end{array}\right]
$$

V and I represent the terminal voltages and currents, respectively, in the Laplacedomain and $d$ is the length of the line. The MTL system described by (2.2) does not have a direct representation in the time-domain, which makes it difficult to interface with nonlinear transient simulators. In order to overcome this problem, the partial differential equations of (2.1) are approximated by a set of rational functions, which can be converted into a set of equivalent subcircuits for SPICE simulation [3], [7], [9]-[13].

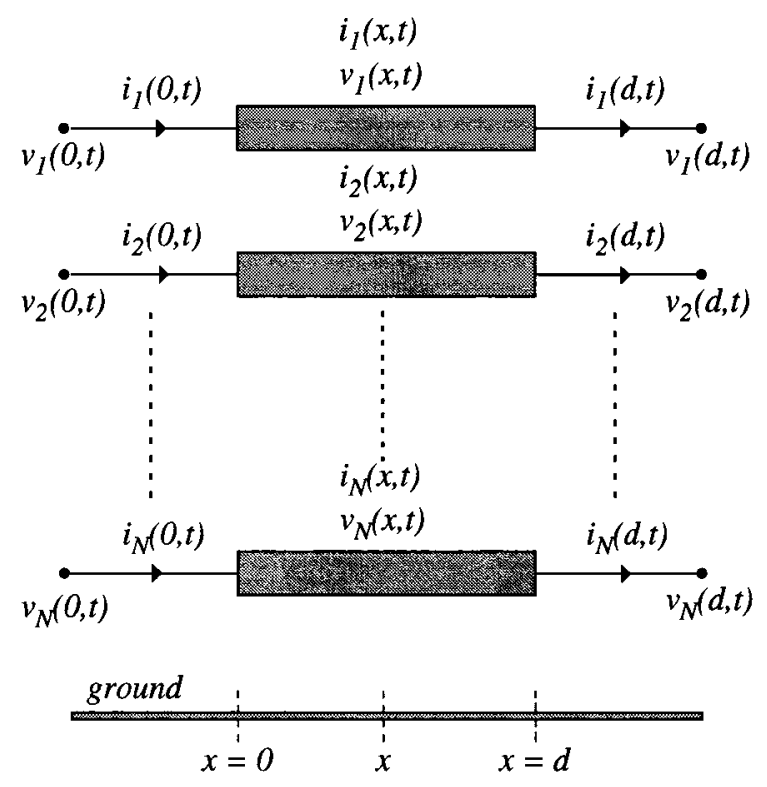

Fig. 2.1: Multiconductor transmission line system. 


\subsubsection{Matrix Rational Approximations (MRA)}

The exponential matrix function can be approximated as [10]

$$
\mathbf{P}_{N, M}(\mathbf{Z}) e^{\mathbf{Z}} \approx \mathbf{Q}_{N, M}(\mathbf{Z})
$$

where $\mathbf{P}_{N, M}(\mathbf{Z})$ and $\mathbf{Q}_{N, M}(\mathbf{Z})$ are polynomial matrices of order $N$ and $M$, respectively, defined by the closed-form Padé expressions [12]

$$
\begin{aligned}
& \mathbf{P}_{N, M}(\mathbf{Z})=\sum_{i=0}^{N} \frac{(M+N-i) ! N !}{(M+N) ! i !(N-i) !}(-\mathbf{Z})^{i}, \\
& \mathbf{Q}_{N, M}(\mathbf{Z})=\sum_{i=0}^{M} \frac{(M+N-i) ! M !}{(M+N) ! i !(M-i) !}(\mathbf{Z})^{i}
\end{aligned}
$$

It was shown in [10] that if $N=M$, then the passivity of the resulting macromodel is maintained. It was also shown that the matrix rational approximation could be substituted into (2.2) to obtain

$$
\left[\begin{array}{c}
\mathbf{V}(d, s) \\
\mathbf{I}(d, s)
\end{array}\right] \approx\left[\mathbf{Q}_{N, N}(-\mathbf{Z})\right]^{-1} \mathbf{Q}_{N, N}(\mathbf{Z})\left[\begin{array}{c}
\mathbf{V}(0, s) \\
\mathbf{I}(0, s)
\end{array}\right]
$$

The Padé rational functions can be represented in terms of subsections, described by pole-zero pairs as

$$
\begin{aligned}
{\left[\mathbf{Q}_{N, N}(-\mathbf{Z})\right]^{-1} \mathbf{Q}_{N, N}(\mathbf{Z}) } & =\prod_{i=1}^{\frac{N}{2}}\left[\mathbf{Q}_{N, N}(-\mathbf{Z})_{i}\right]^{-1} \mathbf{Q}_{N, N}(\mathbf{Z})_{i} \\
& =\prod_{i=1}^{\frac{N}{2}}\left[\left(a_{i} \mathbf{U}-\mathbf{Z}\right)\left(a_{i}^{*} \mathbf{U}-\mathbf{Z}\right)\right]^{-1} \times\left[\left(a_{i} \mathbf{U}+\mathbf{Z}\right)\left(a_{i}^{*} \mathbf{U}+\mathbf{Z}\right)\right]
\end{aligned}
$$


for even values of $N$ and

$$
\begin{aligned}
{\left[\mathbf{Q}_{N, N}(-\mathbf{Z})\right]^{-1} \mathbf{Q}_{N, N}(\mathbf{Z})=} & \prod_{i=1}^{\frac{N+1}{2}}\left[\mathbf{Q}_{N, N}(-\mathbf{Z})_{i}\right]^{-1} \mathbf{Q}_{N, N}(\mathbf{Z})_{i} \\
= & {\left[a_{0} \mathbf{U}-\mathbf{Z}\right]^{-1}\left[a_{0}^{*} \mathbf{U}-\mathbf{Z}\right] \times \prod_{i=1}^{\frac{N-1}{2}}\left[\left(a_{i} \mathbf{U}-\mathbf{Z}\right)\left(a_{i}^{*} \mathbf{U}-\mathbf{Z}\right)\right]^{-1} } \\
& \times\left[\left(a_{i} \mathbf{U}+\mathbf{Z}\right)\left(a_{i}^{*} \mathbf{U}+\mathbf{Z}\right)\right]
\end{aligned}
$$

for odd values of $N$. In this case, $\mathbf{Z}$ is defined as $\mathbf{Z}=(\mathbf{D}(s)+s \mathbf{E}(s)) d, \mathbf{U}$ represents

the unity matrix, and $a_{k} ; k \geq 0$, are predetermined coefficients [13]. The symbol * represents the complex conjugate operation.

Equations (2.8) and (2.9) are converted into a set of equivalent subcircuits for integration in SPICE-like simulators [13]. The advantage of this process is that the macromodel is passive by construction [10]. The following section discusses the key issues of passivity, causality, and high-order networks in interconnect macromodeling.

\subsection{Macromodeling Issues}

Rational function approximation based macromodeling techniques for networks characterized by tabulated data have been very successful in obtaining stable and accurate transient simulations [30], [36]. However, several issues concerning macromodel properties have arisen. Since these issues can seriously affect the accuracy of transient SPICE simulations, they need to be discussed.

First, all macromodels describing stable systems must also be stable. A stable system has all its poles in the LHP and will produce bounded output responses for 
any bounded input. An unstable response to a bounded input is illustrated in Fig. 2.2 , producing spurious oscillations in the output as time approaches infinite. Since rational approximation algorithms have been developed to effectively obtain stable poles [30], [36], this property is not discussed further.

Second, a macromodel representing a passive and causal structure, needs to be both passive and causal [23]-[26], [38]-[40], [53]. Third, for long delay lines, the order of rational approximations can become very high, leading to large macromodels and subsequently, prohibitively CPU expensive transient simulations. A review of passivity, causality, and long delay issues is given below.

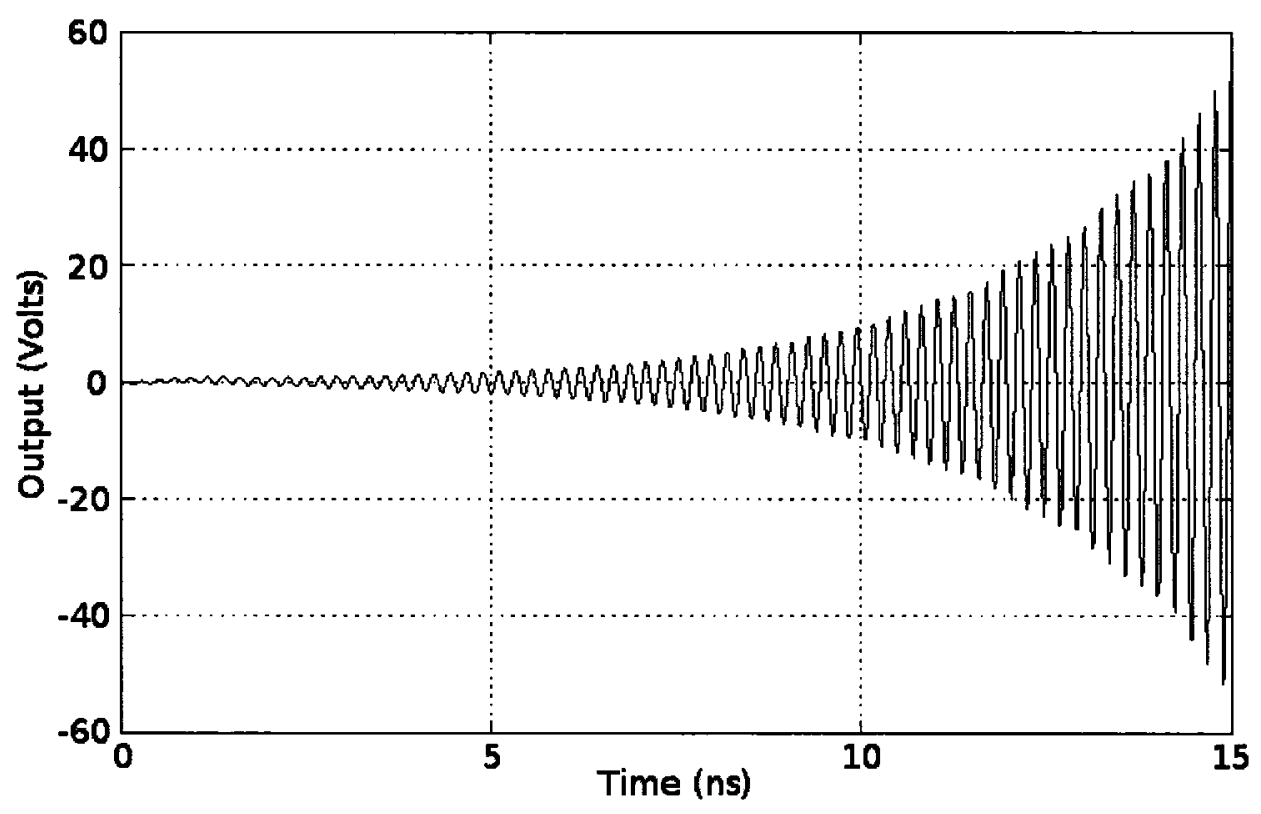

Fig. 2.2: Transient response of a stable, but non-passive macromodel with passive terminations. 


\subsubsection{Passivity}

Rational function based approximation algorithms produce accurate and stable models representing networks characterized by tabulated data [30], [36]. However, these models may fail to guarantee the important physical property of passivity. A passive network is one that cannot generate more energy than it absorbs [54]. Passivity is important because a stable, but non-passive system can become unstable when connected to other passive devices [3], [41], [44].

In order to illustrate this concept, consider the single port network shown in Fig. 2.3(a). The poles of the transfer function for the network are clearly in the LHP, therefore it is a stable system, but not necessarily passive. When a passive load is connected to the network, the poles of the transfer function for the network (shown in Fig. 2.3(b)) now lie in the RHP. Thus, the complete system is unstable, even though each network, seperately, is stable. This problem can be overcome by creating macromodels that are passive since all passive components connected to other passive components are (collectively) passive and stable [13].

It is important to note that a stable system is not necessarily passive; however, all passive systems are stable. Therefore, it is sufficient to satisfy passivity conditions to obtain both a stable and passive macromodel. The necessary and sufficient passivity conditions for admittance parameters are the following [55]

1. $\mathbf{Y}\left(s^{*}\right)=\mathbf{Y}^{*}(s)$ for all complex $s$, where ${ }^{*}$ is the complex conjugate operator,

2. $\mathbf{Y}(s)$ is positive real, that is, $z^{* T}\left(\mathbf{Y}(s)+\mathbf{Y}^{T}\left(s^{*}\right)\right) z \geq 0$ for all complex values of $s$ satisfying $\mathfrak{R e}(s) \geq 0$ and for any complex vector $z$. 


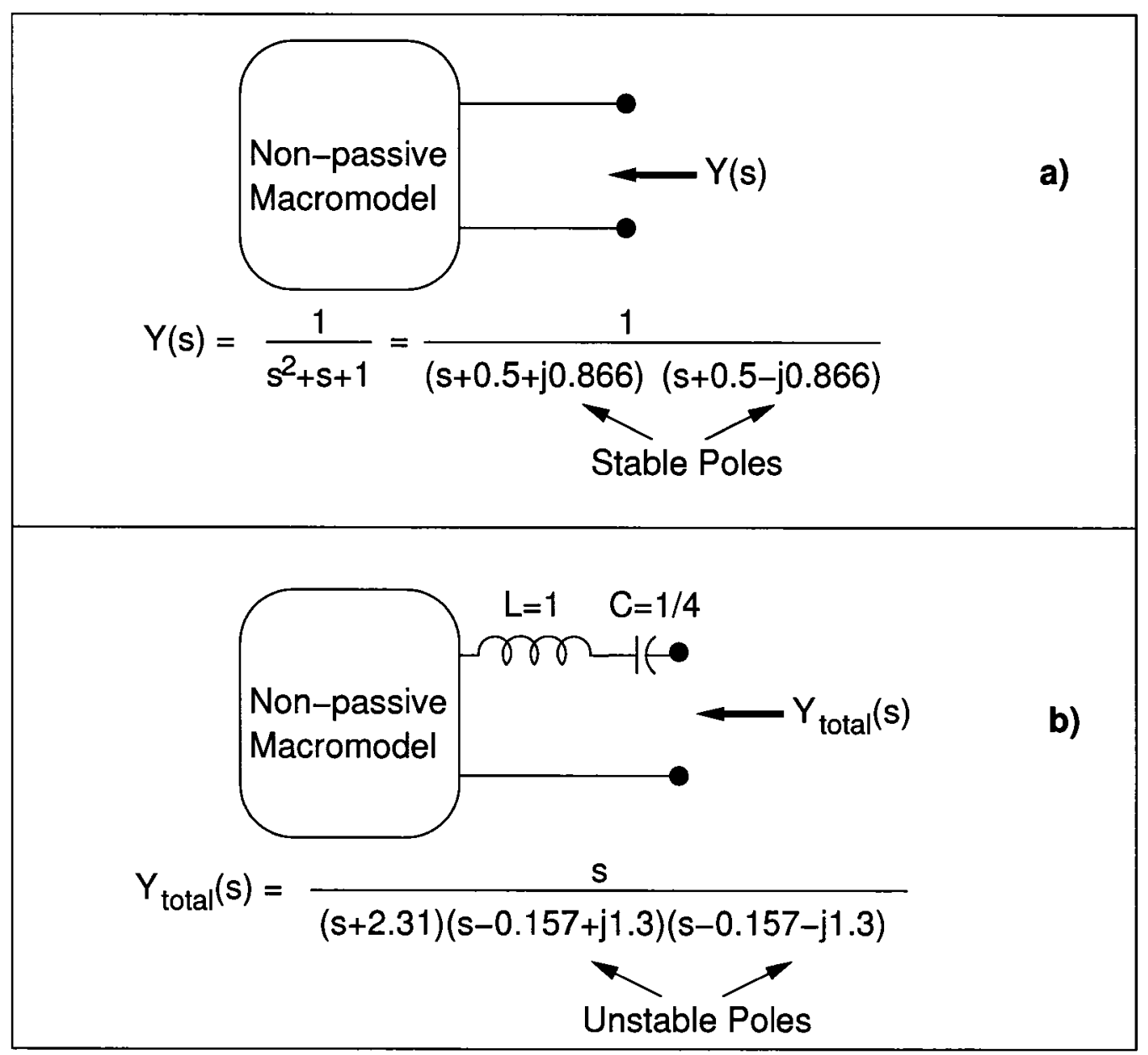

Fig. 2.3: Illustration of significance of passivity: a) Stable-non-passive macromodel b) Unstable macromodel with passive loads.

\subsubsection{Causality}

A causal system is one such that its response satisfies

$$
h(t)=0 \quad \text { for } t<0 .
$$


Causality is an important concept to consider since macromodels constructed from causal devices should also be causal [23]-[26]. In the case of linear systems, it was shown that if the system is passive, then it is also causal [54]. Therefore, it is sufficient to ensure that the macromodel of a passive linear device is passive to obtain a stable, causal, and passive macromodel.

\subsubsection{Long Delay Networks}

As discussed in section 1, long delays are fast becoming one of the biggest issues concerning interconnect macromodeling [7]-[13], [29], [45]-[47]. Delays are characterized in the frequency-domain by the exponential function $e^{-s \tau}$, where $\tau$ is the delay in the network. However, conventional macromodeling techniques [30], [36] produce rational approximations in the form

$$
f(s)=\sum_{i=1}^{M} \frac{k_{i}}{s-p_{i}}+d+s h
$$

where $k_{i}$ and $p_{i}$ are real or come in complex conjugate pairs representing the residues and poles, respectively. The values $d$ and $h$ are both real, and $M$ is the number of poles.

The exponential function has an infinite number of poles and zeros, while rational approximations contain only a finite number of each. Therefore, for high frequencies and/or long delays, the rational approximation of a single delay element can require hundreds of poles to obtain an accurate approximation. Consequently, when highorder rational functions are converted into an equivalent circuit for SPICE simulation, they require a large number of circuit elements and subsequently, become stiff ODEs 
requiring small time-steps for accurate transient simulations.

\subsection{DEPACT Macromodeling Algorithm}

For long delay lines, the MRA algorithm was found to produce high-order rational function approximations, leading to large macromodels and slow transient simulation times. In order to overcome this difficulty, the DEPACT algorithm was developed $[7]-[9]$.

The delay portion of the system is primarily contained in the matrix $\mathbf{E}$. Therefore, extracting the delay from the exponential system can be done by separating the $s \mathbf{E}$ term from the exponential matrix function, $e^{\mathbf{D}+s \mathbf{E}}$. Unfortunately, since the matrices $\mathbf{D}$ and $s \mathbf{E}$ do not necessarily commute, $e^{\mathbf{D}+s \mathbf{E}} \neq e^{\mathbf{D}} e^{s \mathbf{E}}$. Therefore, an approximation is required for $e^{\mathrm{D}+s \mathrm{E}}$.

The Lie product gives an approximation for this function, along with an error estimation, $\epsilon_{m}[9]$. This formula is

$$
e^{\mathbf{D}+s \mathbf{E}} \cong \prod_{k=1}^{m} \Psi_{k}+\epsilon_{m}, \quad \Psi_{k} \equiv e^{\frac{s \mathbf{E}}{m}} e^{\frac{\mathbf{D}}{m}}
$$

where $m$ is the number of terms in the product, and $\left\|\epsilon_{m}\right\| \cong O(1 / m)$ is the order of the error. The problem with this formulation is that it does not maintain the symmetry of the transmission line circuit, and the error decays with $O(1 / m)$. Therefore, a large value of $m$ may be required to achieve the desired accuracy. This problem is alleviated by modifying the Lie product approximation to obtain [7]

$$
e^{\mathbf{D}+s \mathbf{E}} \cong \prod_{k=1}^{m} \boldsymbol{\Psi}_{k}+\epsilon_{m}, \quad \boldsymbol{\Psi}_{k} \equiv e^{\frac{\mathbf{D}}{2 m}} e^{\frac{s \mathbf{E}}{m}} e^{\frac{\mathbf{D}}{2 m}}
$$


where the error is now given by $\left\|\epsilon_{m}\right\| \cong O\left(1 / m^{2}\right)$. In the case of long lines with $\|\mathbf{D}\| \leq\|s \mathbf{E}\|$, the Lie product can be improved further using the approximation

$$
e^{\mathbf{D}+s \mathbf{E}} \cong \prod_{k=1}^{m} \boldsymbol{\Psi}_{k}+\epsilon_{m}, \quad \mathbf{\Psi}_{k} \equiv e^{\frac{s \mathbf{E}}{2 m}} e^{\frac{\mathbf{D}}{m}} e^{\frac{s \mathbf{E}}{2 m}}
$$

where the error $\epsilon_{m} \cong O\left(1 / m^{2}\right)$ is smaller than in (2.13). This formula can be improved further by taking the average of (2.13) and (2.14) to obtain

$$
e^{\mathbf{D}+s \mathbf{E}} \cong \prod_{k=1}^{m} \Psi_{k}+\epsilon_{m}, \quad \Psi_{k} \equiv \frac{1}{2}\left(e^{\frac{s \mathbf{E}}{2 m}} e^{\frac{\mathbf{D}}{m}} e^{\frac{s \mathbf{E}}{2 m}}+e^{\frac{\mathbf{D}}{2 m}} e^{\frac{s \mathbf{E}}{m}} e^{\frac{\mathbf{D}}{2 m}}\right)
$$

where the error, $\epsilon_{m} \cong O\left(1 / m^{2}\right)$, is even smaller than in (2.14).

\subsubsection{Frequency Dependent Parameters}

It is common for the p.u.l parameters of a line to be a function of frequency, since at high frequencies these parameters can no longer be approximated as constant. The maximum delay that can be extracted without affecting the passivity of the model was found to be $\mathbf{E}(\infty)[7]$. Let

$$
\mathbf{E}_{a}(s)=\mathbf{E}(s)-\mathbf{E}(\infty)=\left[\begin{array}{cc}
\mathbf{0} & -\mathbf{L}_{a}(s) \\
-\mathbf{C}_{a}(s) & \mathbf{0}
\end{array}\right]
$$

such that $\mathbf{L}_{a}(s)$ and $\mathbf{C}_{a}(s)$ are positive definite matrices. The MLF relations (2.13)(2.15) can be applied to the frequency dependent matrix exponential solution for 
distributed transmission lines, $e^{\mathbf{D}(s)+s \mathbf{E}(s)}$. For (2.14), this yields

$$
e^{\mathbf{D}(s)+s \mathbf{E}(s)} \approx \prod_{k=1}^{m}\left(e^{\frac{s \mathbf{E}(\infty)}{2 m}} e^{\frac{\mathbf{D}(s)+s \mathbf{E}_{a}(s)}{m}} e^{\frac{s \mathbf{E}(\infty)}{2 m}}\right)
$$

\subsubsection{Estimation of the Order of Approximation}

In order to determine the order of the approximation, the maximum allowable error is defined as

$$
E_{\text {max }}=\max _{0 \leq f \leq f_{\max }}\left\|e^{\mathbf{D}+s \mathbf{E}}-\prod_{k=1}^{m} \Psi_{k}\right\|,
$$

where $f_{\max }$ is the maximum frequency of interest. The order of approximation, $m$, can be determined from (2.18) using $f_{\text {max }}$, which is usually obtained from the rise/fall times of the input signal as [3]

$$
f_{\text {max }} \approx \frac{0.35}{\text { Rise Time }}
$$

\subsection{Macromodeling Algorithms for Interconnects Characterized by Tabulated Data}

As it becomes increasingly difficult to obtain analytical models for high-speed interconnects, macromodeling networks characterized by tabulated data is fast becoming an integral part of accurate signal integrity analysis in high-speed designs. Rational function based macromodeling techniques are the most common methods for modeling tabulated/measured data [18]-[42]. Using these approaches, the tabulated data 
is approximated with a function defined in the frequency-domain as

$$
Y(s)=\frac{a_{0}+a_{1} s^{1}+a_{2} s^{2}+\ldots+a_{m-1} s^{m-1}+a_{m} s^{m}}{1+b_{1} s^{1}+b_{2} s^{2}+\ldots+b_{n-1} s^{n-1}+b_{n} s^{n}}
$$

where the coefficients $a_{0}, a_{1}, \ldots, a_{m}$ and $b_{1}, b_{2}, \ldots, b_{n}$ need to be determined. Subsequently, the rational approximation is converted into an equivalent circuit representation for SPICE simulation.

The following sections outline the rational function approximation algorithms most relevant to this thesis. These include the FD-VF algorithm [30], the TD-VF algorithm [36], and Prony's algorithm [56], respectively.

\subsubsection{Frequency-Domain Vector Fit (FD-VF)}

The FD-VF algorithm begins with a function represented by a set of data of $N$ points, $\{f(s)\}=\left\{f\left(s_{1}\right), f\left(s_{2}\right), \ldots, f\left(s_{N}\right)\right\}$ and builds a rational approximation of the form $[30]$

$$
f(s)=\sum_{i=1}^{M} \frac{k_{i}}{s-p_{i}}+d+s h
$$

where $k_{i}$ and $p_{i}$ are real or come in complex conjugate pairs representing the residues and poles, respectively. The values $d$ and $h$ are both real, and $M$ is the number of poles. The goal of the FD-VF algorithm is to estimate all the coefficients in (2.21) so that a least squares approximation of $f(s)$ is obtained over a given frequency interval.

The FD-VF algorithm solves the problem in (2.21) through a linear formulation using two stages. Stage one makes an initial guess at the poles over the frequency interval of interest. Next, it iterates on the initial guess of the poles by solving a least squares problem for an unknown function, $\sigma(s)$, whose zeros become the new poles 
of the system. Stage two then calculates the residues of (2.21) using the final set of poles from stage one.

First, the details of the algorithm using real poles are explained. Following this, a robust choice of starting poles are defined, and modifications for complex poles poles and multi-port networks are examined.

\section{Stage 1 : Pole Identification}

The algorithm begins by choosing a set of starting poles, $\left\{\bar{p}_{i}\right\}$. Following this, a scaling function, $\sigma(s)$, is defined such that

$$
\sigma(s)=\sum_{i=1}^{M} \frac{\tilde{k}_{i}}{s-\bar{p}_{i}}+1
$$

where $\tilde{k}_{i}$ are the residues of $\sigma(s)$. Next, a scaled function is defined such that

$$
\sigma(s) f(s) \approx \hat{f}(s)=\sum_{i=1}^{M} \frac{\hat{k}_{i}}{s-\bar{p}_{i}}+\hat{d}+s \hat{h}
$$

Equations (2.22) and (2.23) are combined to obtain

$$
\left[\sum_{i=1}^{M} \frac{\hat{k}_{i}}{s-\bar{p}_{i}}+\hat{d}+s \hat{h}\right] \approx\left[\sum_{i=1}^{M} \frac{\tilde{k}_{i}}{s-\bar{p}_{i}}+1\right] f(s)
$$

or

$$
\left[\sum_{i=1}^{M} \frac{\hat{k}_{i}}{s-\bar{p}_{i}}+\hat{d}+s \hat{h}\right]-\left[\sum_{i=1}^{M} \frac{\tilde{k}_{i}}{s-\bar{p}_{i}}\right] f(s)=f(s) .
$$

Writing (2.25) at $N$ frequency points, where $N$ is greater than or equal to $2 M+2$ (the number of unknowns), results in an overdetermined linear least squares problem 
of the form

$$
\mathbf{A} \boldsymbol{x}=\boldsymbol{b}
$$

where the unknowns $\hat{k}_{i}, \hat{d}, \hat{h}$, and $\tilde{k}_{i}$ are contained in the solution vector $\boldsymbol{x}$. This is expanded as

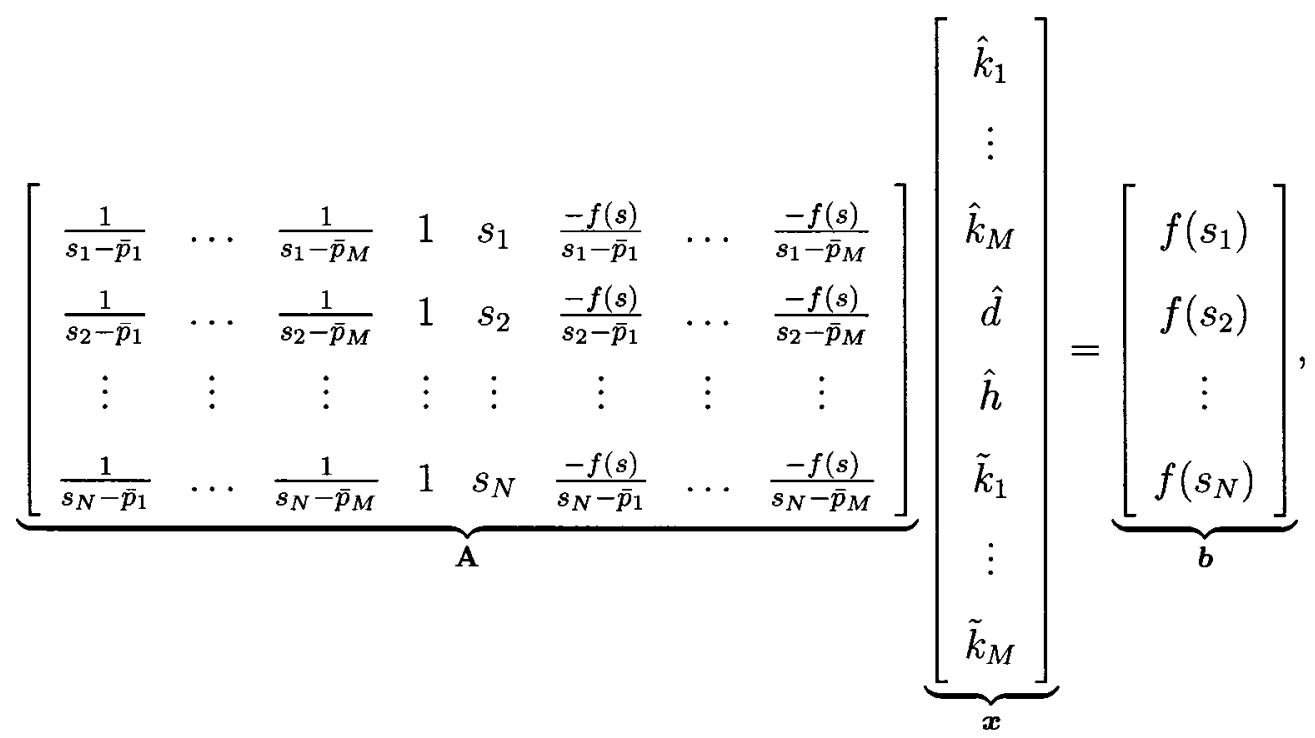

where all the unknowns $\hat{k}_{i}, \hat{d}, \hat{h}$, and $\tilde{k}_{i}$ are real provided the poles are real. This system can be solved using the standard Householder algorithm [57].

Equation (2.25) is now used to obtain a rational function approximation for the function $f(s)$. Let $\left\{\hat{z}_{i}\right\}$ and $\left\{\tilde{z}_{i}\right\}$ be the zeros of $\hat{f}(s)$ and $\sigma(s)$, respectively. Then, note that the partial fraction expansion of $\sigma(s)$ and $f(s)$ can be written as

$$
\hat{f}(s)=\hat{h} \frac{\prod_{i=1}^{M+1}\left(s-\hat{z}_{i}\right)}{\prod_{i=1}^{M}\left(s-\bar{p}_{i}\right)}, \quad \sigma(s)=\frac{\prod_{i=1}^{M}\left(s-\tilde{z}_{i}\right)}{\prod_{i=1}^{M}\left(s-\bar{p}_{i}\right)}
$$


Substituting (2.28) into (2.23), we get

$$
f(s)=\frac{\hat{f}(s)}{\sigma(s)}=\hat{h} \frac{\left(\prod_{i=1}^{M+1}\left(s-\hat{z}_{i}\right)\right) /\left(\prod_{i=1}^{M}\left(s-\bar{p}_{i}\right)\right)}{\left(\prod_{i=1}^{M}\left(s-\tilde{z}_{i}\right)\right) /\left(\prod_{i=1}^{M}\left(s-\bar{p}_{i}\right)\right)}=\hat{h} \frac{\left(\prod_{i=1}^{M+1}\left(s-\hat{z}_{i}\right)\right)}{\left(\prod_{i=1}^{M}\left(s-\tilde{z}_{i}\right)\right)}
$$

Therefore, the zeros of $\sigma(s)$ represent a refined set of poles for the function $f(s)$. This stage is repeated with the zeros of $\sigma(s)$ taken as the new set of starting poles until the desired convergence criteria is obtained. In some instances, the poles determined using (2.29) will lie in the RHP, meaning they are unstable. This problem is overcome by inverting the signs of the real parts for the unstable poles and performing another iteration.

Calculation of Zeros: The zeros of the function, $\sigma(s)$ can also be obtained by determining the eigenvalues of the matrix [30]

$$
\mathbf{H}=\Psi-g \tilde{\phi}^{T}
$$

where

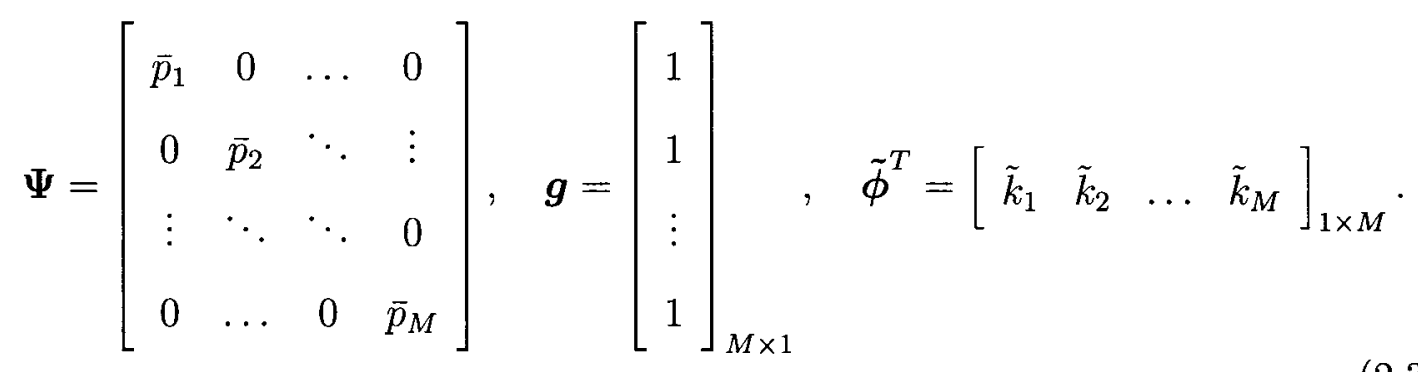


The matrix $\Psi$ is a diagonal matrix containing the starting poles, $\boldsymbol{g}$ is a vector of ones, and $\tilde{\phi}$ is a row vector containing the residues of $\sigma(s)$.

\section{Stage 2 : Residue Identification}

In this stage, the residues of $f(s)$ are calculated using the final set of poles, $\left\{p_{i}\right\}$, found in stage one. This yields an overdetermined least squares problem similar to (2.27), but instead it solves (2.21) at $N$ frequency points. Then, writing this in the form $\mathbf{A} \boldsymbol{x}=\boldsymbol{b}$ yields

$$
\underbrace{\left[\begin{array}{ccccc}
\frac{1}{s_{1}-p_{1}} & \cdots & \frac{1}{s_{1}-p_{M}} & 1 & s_{1} \\
\frac{1}{s_{2}-p_{1}} & \cdots & \frac{1}{s_{2}-p_{M}} & 1 & s_{2} \\
\vdots & \vdots & \vdots & \vdots & \vdots \\
\frac{1}{s_{N}-p_{1}} & \cdots & \frac{1}{s_{N}-p_{M}} & 1 & s_{N}
\end{array}\right]}_{\mathbf{A}} \underbrace{\left[\begin{array}{c}
k_{1} \\
\vdots \\
k_{M} \\
d \\
h
\end{array}\right]}_{\boldsymbol{x}}=\underbrace{\left[\begin{array}{c}
f\left(s_{1}\right) \\
f\left(s_{2}\right) \\
\vdots \\
f\left(s_{N}\right)
\end{array}\right]}_{\boldsymbol{b}} .
$$

\section{Selection of Starting Poles}

Functions with distinct resonance peaks should be modeled using complex poles. In this case, the initial set of poles for the algorithm consists of imaginary parts, $\beta$, linearly distributed over the frequency range of interest. These poles are chosen in complex conjugate pairs such that

$$
p_{i}=-\alpha+j \beta, \quad p_{i+1}=-\alpha-j \beta
$$


where

$$
\alpha=\beta / 100
$$

is chosen to be small to avoid ill-conditioning in the least squares problems [30].

Smooth functions are best modeled using real poles linearly or logarithmically spaced as a function of frequency.

\section{Modifications for Complex Poles}

In order to ensure that the residues from the least squares computations in (2.27) and (2.32) come in complex conjugate pairs, the matrix formulations are modified. If the partial fractions $i$ and $i+1$ correspond to complex pairs, then we have $\frac{M}{2}$ complex pairs where

$$
\begin{array}{ll}
p_{i}=\mathfrak{R e}\left(p_{i}\right)+j \mathfrak{I m}\left(p_{i}\right), & p_{i+1}=\mathfrak{R e}\left(p_{i}\right)-j \mathfrak{I m}\left(p_{i}\right), \\
k_{i}=\mathfrak{R e}\left(k_{i}\right)+j \mathfrak{I m}\left(k_{i}\right), & k_{i+1}=\mathfrak{R e}\left(k_{i}\right)-j \mathfrak{I m}\left(k_{i}\right) .
\end{array}
$$

Equation (2.27) is written in the form $\mathbf{A} \boldsymbol{x}=\boldsymbol{b}$, where

$$
\mathbf{A}=\left[\begin{array}{llllll}
\mathbf{A}_{1} & \mathbf{A}_{2} & \ldots & \mathbf{A}_{\frac{M}{2}} & \mathbf{1} & \mathbf{S}
\end{array}\right], \quad \mathbf{1}=\left[\begin{array}{c}
1 \\
1 \\
\vdots \\
1
\end{array}\right]_{N \times 1}, \quad \mathbf{S}=\left[\begin{array}{c}
s_{1} \\
s_{2} \\
\vdots \\
s_{N}
\end{array}\right]
$$


and,

$$
\begin{gathered}
\mathbf{A}_{\mathbf{i}}=\left[\begin{array}{cccc}
\left(\frac{1}{s_{1}-\bar{p}_{i}}+\frac{1}{s_{1}-\bar{p}_{i}^{*}}\right) & \left(\frac{j}{s_{1}-\bar{p}_{i}}-\frac{j}{s_{1}-\bar{p}_{i}^{*}}\right) & \left(\frac{f\left(s_{1}\right)}{s_{1}-\bar{p}_{i}}+\frac{f\left(s_{1}\right)}{s_{1}-\bar{p}_{i}^{*}}\right) & \left(\frac{j f\left(s_{1}\right)}{s_{1}-\bar{p}_{i}}-\frac{j f\left(s_{1}\right)}{s_{1}-\bar{p}_{i}^{*}}\right) \\
\left(\frac{1}{s_{2}-\bar{p}_{i}}+\frac{1}{s_{2}-\bar{p}_{i}^{*}}\right) & \left(\frac{j}{s_{2}-\bar{p}_{i}}-\frac{j}{s_{2}-\bar{p}_{i}^{*}}\right) & \left(\frac{f\left(s_{2}\right)}{s_{2}-\bar{p}_{i}}+\frac{f\left(s_{2}\right)}{s_{2}-\bar{p}_{i}^{*}}\right) & \left(\frac{j f\left(s_{2}\right)}{s_{2}-\tilde{p}_{i}}-\frac{j f\left(s_{2}\right)}{s_{2}-\bar{p}_{i}^{*}}\right) \\
\vdots & \vdots & \vdots \\
\left(\frac{1}{s_{N}-\bar{p}_{i}}+\frac{1}{s_{N}-\bar{p}_{i}^{*}}\right) & \left(\frac{j}{s_{N}-\bar{p}_{i}}-\frac{j}{s_{N}-\bar{p}_{i}^{*}}\right) & \left(\frac{f\left(s_{N}\right)}{s_{N}-\bar{p}_{i}}+\frac{f\left(s_{N}\right)}{s_{N}-\bar{p}_{i}^{*}}\right) & \left(\frac{j f\left(s_{N}\right)}{s_{N}-\bar{p}_{i}}-\frac{j f\left(s_{N}\right)}{s_{N}-\tilde{p}_{i}^{*}}\right)
\end{array}\right], \\
\boldsymbol{x}=\left[\begin{array}{cccc}
\mathfrak{R e}\left(\hat{k}_{1}\right) \mathfrak{I m}\left(\hat{k}_{1}\right) \mathfrak{R e}\left(\tilde{k}_{1}\right) \mathfrak{I m}\left(\tilde{k}_{1}\right) \ldots \mathfrak{R e}\left(\hat{k}_{\frac{M}{2}}\right) \mathfrak{I m}\left(\hat{k}_{\frac{M}{2}}\right) \mathfrak{R e}\left(\tilde{k}_{\frac{M}{2}}\right) \mathfrak{I m}\left(\tilde{k}_{\frac{M}{2}}\right) \hat{d} \hat{h}
\end{array}\right]^{T}, \\
\boldsymbol{b}=\left[\begin{array}{cccc}
f\left(s_{1}\right) & f\left(s_{2}\right) & \ldots & f\left(s_{N}\right)
\end{array}\right]^{T} .
\end{gathered}
$$

Similarly, for the least squares problem $(\mathbf{A} \boldsymbol{x}=\boldsymbol{b})$ from $(2.32)$, the variables become

$$
\begin{aligned}
& \mathbf{A}=\left[\begin{array}{llllll}
\mathbf{A}_{1} & \mathbf{A}_{2} & \ldots & \mathbf{A}_{\frac{M}{2}} & \mathbf{1} & \mathbf{S}
\end{array}\right], \quad \mathbf{1}=\left[\begin{array}{c}
1 \\
1 \\
\vdots \\
1
\end{array}\right]_{N \times 1}, \quad \mathbf{S}=\left[\begin{array}{c}
s_{1} \\
s_{2} \\
\vdots \\
s_{N}
\end{array}\right], \\
& \mathbf{A}_{\mathbf{i}}=\left[\begin{array}{cc}
\left(\frac{1}{s_{1}-\bar{p}_{i}}+\frac{1}{s_{1}-\bar{p}_{i}^{*}}\right) & \left(\frac{j}{s_{1}-\bar{p}_{i}}-\frac{j}{s_{1}-\bar{p}_{i}^{*}}\right) \\
\left(\frac{1}{s_{2}-\bar{p}_{i}}+\frac{1}{s_{2}-\bar{p}_{i}^{*}}\right) & \left(\frac{j}{s_{2}-\bar{p}_{i}}-\frac{j}{s_{2}-\bar{p}_{i}^{*}}\right) \\
\vdots & \vdots \\
\left(\frac{1}{s_{N}-\bar{p}_{i}}+\frac{1}{s_{N}-\tilde{p}_{i}^{*}}\right) & \left(\frac{j}{s_{N}-\bar{p}_{i}}-\frac{j}{s_{N}-\bar{p}_{i}^{*}}\right)
\end{array}\right] \\
& \boldsymbol{x}=\left[\begin{array}{lllllll}
\mathfrak{R e}\left(k_{1}\right) & \mathfrak{I m}\left(k_{1}\right) & \ldots & \mathfrak{R e}\left(k_{\frac{M}{2}}\right) & \mathfrak{I m}\left(k_{\frac{M}{2}}\right) & d & h
\end{array}\right]^{T}, \\
& \boldsymbol{b}=\left[\begin{array}{llll}
f\left(s_{1}\right) & f\left(s_{2}\right) & \ldots & f\left(s_{N}\right)
\end{array}\right]^{T} .
\end{aligned}
$$


The overdetermined least squares problems in (2.38) and (2.39) are formulated in terms of real quantities to preserve complex conjugate pairings. The problem becomes

$$
\underbrace{\left[\begin{array}{c}
\mathfrak{R e}(\mathbf{A}) \\
\mathfrak{I m}(\mathbf{A})
\end{array}\right]}_{\hat{\mathbf{A}}} \boldsymbol{x}=\underbrace{\left[\begin{array}{c}
\mathfrak{R e}(\boldsymbol{b}) \\
\mathfrak{I m}(\boldsymbol{b})
\end{array}\right]}_{\hat{\boldsymbol{b}}}
$$

or

$$
\hat{\mathbf{A}} \boldsymbol{x}=\hat{b}
$$

which is solved using the standard Householder algorithm.

Calculation of Zeros for Complex Poles: In the case of complex poles, the calculation of zeros from (2.30) needs to be modified as well. The zeros are now the eigenvalues of the system

$$
\mathbf{H}=\Psi-g \tilde{\phi}^{T}
$$

where

$$
\begin{aligned}
& \boldsymbol{\Psi}=\left[\begin{array}{cccc}
\boldsymbol{\Psi}_{1} & 0 & \ldots & 0 \\
0 & \boldsymbol{\Psi}_{2} & \ddots & \vdots \\
\vdots & \ddots & \ddots & 0 \\
0 & \ldots & 0 & \boldsymbol{\Psi}_{\frac{\mathrm{M}}{2}}
\end{array}\right], \quad \boldsymbol{\Psi}_{\mathbf{i}}=\left[\begin{array}{cc}
\mathfrak{R e}\left(\bar{p}_{i}\right) & \mathfrak{I m}\left(\bar{p}_{i}\right) \\
-\mathfrak{I m}\left(\bar{p}_{i}\right) & \mathfrak{R e}\left(\bar{p}_{i}\right)
\end{array}\right], \quad i=1,2, \ldots, \frac{M}{2},
\end{aligned}
$$

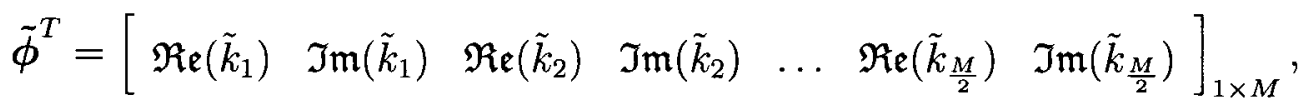

$$
\begin{aligned}
& \boldsymbol{g}=\left[\begin{array}{lllllll}
2 & 0 & 2 & 0 & \ldots & 2 & 0
\end{array}\right]_{M \times 1}^{T} .
\end{aligned}
$$


This modifies $\Psi$ to become a real matrix with eigenvalues corresponding to the real and imaginary parts of the complex zeros in $\sigma(s)$.

\section{Common Pole Sets for Multiport Networks}

The FD-VF algorithm detailed in the previous sections was for a scalar function. If the pole-sets are calculated for each individual matrix element, then a large number of redundant poles could result, leading to inefficient transient simulations [38]. Therefore, in order to minimize the number of poles in the matrix-transfer functions, the following two propositions are used [6].

1. In general, the pole set corresponding to any individual admittance element is a subset of the union of all driving point admittances [58].

2. Generally, in a system with a large number of dominant poles, pole sets belonging to different driving point admittances contain mostly identical poles and only a very small percentage of poles differ among these sets.

A common pole set for a multiport network is obtained by performing the pole identification process described above on driving point admittances. The initial set of poles is found by first running the pole finding iterations on a single driving point admittance, resulting in faster convergence for the pole finding algorithm of the driving point functions [41].

It should be noted that while a common pole set can be found for admittance parameters, it has not been shown to be possible on scattering parameters. 


\subsubsection{Time-Domain Vector Fit (TD-VF)}

The TD-VF algorithm is applied to data for package structures with $P$ ports [36], [37]. The data for the system is obtained by exciting each port, $j$, with a function, $\left\{x_{j}(t)\right\}=\left\{x_{j}\left(t_{1}\right), x_{j}\left(t_{2}\right), \ldots, x_{j}\left(t_{n}\right)\right\}$, and measuring the output responses, $\left\{y_{i j}(t)\right\}=$ $\left\{y_{i j}\left(t_{1}\right), y_{i j}\left(t_{2}\right), \ldots, y_{i j}\left(t_{n}\right)\right\}$, at port $i$. The algorithm builds a matrix transfer function in rational form

$$
\mathbf{H}(s)=\mathbf{D}+\sum_{m=1}^{M} \frac{\mathbf{K}_{m}}{s-p_{m}},
$$

where $\mathbf{K}_{m}$ and $p_{m}$ are real or complex conjugate pairs representing the residues and poles, respectively. $\mathbf{D}$ is a purely real matrix containing the direct coupling constants of the system and $M$ is the number of poles in the system. For multiport networks, techniques analogous to those in section 2.5.1 are used to determine the common pole sets.

For simplicity, this section derives the TD-VF algorithm of a scalar network. In this case, only a single set of input data, $\{x(t)\}$, and response data, $\{y(t)\}$, is given at $n$ time points. The final product is an approximation for the transfer function

$$
H(s)=d+\sum_{m=1}^{M} \frac{k_{m}}{s-p_{m}}
$$

where $\left\{k_{m}\right\}$ are the residues, $d$ is the direct coupling constant, and $\left\{p_{m}\right\}$ is the set of $M$ poles in the system. The following sections outline the TD-VF algorithm in detail [36], [37]. 


\section{Stage 1 : Pole Identification}

First, a set of starting poles, $\left\{\bar{p}_{i}\right\}$, is chosen as in FD-VF (the details are given in section 2.5.1). Next, using these poles, define the scalar weight function, $\sigma(s)$, such that

$$
\sigma(s)=\sum_{m=1}^{M} \frac{\tilde{k}_{m}}{s-\bar{p}_{m}}+1
$$

where $\left\{\tilde{k}_{m}\right\}$ are the residues of $\sigma(s)$. In addition, define the scaled transfer function as

$$
\sigma(s) H(s) \approx \hat{H}(s)=\sum_{m=1}^{M} \frac{\hat{k}_{m}}{s-\bar{p}_{m}}+\hat{d} .
$$

The transfer function, $H(s)$, can also be written as

$$
H(s)=\frac{Y(s)}{X(s)}
$$

where $Y(s)$ and $X(s)$ are the Laplace transforms of the output and input functions, respectively. Substituting (2.48) into (2.47) and rearranging yields

$$
Y(s)=\hat{d} X(s)+\sum_{m=1}^{M} \frac{\hat{k}_{m} X(s)}{s-\bar{p}_{m}}-\sum_{m=1}^{M} \frac{\tilde{k}_{m} Y(s)}{s-\bar{p}_{m}}
$$

Taking the inverse Laplace transform of (2.49) yields

$$
y(t)=\hat{d} x(t)+\sum_{m=1}^{M} \hat{k}_{m} x_{m}(t)-\sum_{m=1}^{M} \tilde{k}_{m} y_{m}(t)
$$


where

$$
\begin{aligned}
& x_{m}(t)=\int_{0}^{t} e^{\bar{p}_{m}(t-\tau)} x(\tau) d \tau \\
& y_{m}(t)=\int_{0}^{t} e^{\bar{p}_{m}(t-\tau)} y(\tau) d \tau
\end{aligned}
$$

define convolution integrals. Next, first order linear interpolation is applied to solve the integrals, leading to

$$
x_{m}\left(t_{k+1}\right)=\alpha_{m} x_{m}\left(t_{k}\right)+\beta_{m}^{0} x\left(t_{k+1}\right)+\beta_{m}^{1} x\left(t_{k}\right),
$$

where $\alpha_{m}, \beta_{m}^{0}$, and $\beta_{m}^{1}$ are weights for the first order IIR filter given by

$$
\alpha_{m}=e^{\bar{p}_{m} \Delta t}, \quad \beta_{m}^{0}=\frac{-1-\bar{p}_{m} \Delta t+e^{\bar{p}_{m} \Delta t}}{\bar{p}_{m}^{2} \Delta t}, \quad \beta_{m}^{1}=\frac{-1+\left(\bar{p}_{m} \Delta t-1\right) e^{\bar{p}_{m} \Delta t}}{\bar{p}_{m}^{2} \Delta t}
$$

The output response, $y_{m}(t)$, can be interpolated using the same formulation. Once the convolutions are calculated, the residues, $\left\{\tilde{k}_{m}\right\}$, of the scalar weight function, $\sigma(s)$, are found using a least squares formulation, $\mathbf{A} \boldsymbol{x}=\boldsymbol{b}$, with

$$
\mathbf{A}=\left[\begin{array}{ccccccccc}
x\left(t_{1}\right) & x_{1}\left(t_{1}\right) & x_{2}\left(t_{1}\right) & \ldots & x_{M}\left(t_{1}\right) & -y_{1}\left(t_{1}\right) & -y_{2}\left(t_{1}\right) & \ldots & -y_{M}\left(t_{1}\right) \\
x\left(t_{2}\right) & x_{1}\left(t_{2}\right) & x_{2}\left(t_{2}\right) & \ldots & x_{M}\left(t_{2}\right) & -y_{1}\left(t_{2}\right) & -y_{2}\left(t_{2}\right) & \ldots & -y_{M}\left(t_{2}\right) \\
\vdots & \vdots & \vdots & \ddots & \vdots & \ldots & \ldots & \ddots & \ldots \\
x\left(t_{n}\right) & x_{1}\left(t_{n}\right) & x_{2}\left(t_{n}\right) & \ldots & x_{M}\left(t_{n}\right) & -y_{1}\left(t_{n}\right) & -y_{2}\left(t_{n}\right) & \ldots & -y_{M}\left(t_{n}\right)
\end{array}\right]
$$


and,

$$
\begin{gathered}
\boldsymbol{x}=\left[\begin{array}{ccccccccc}
\hat{d} & \hat{k}_{1} & \hat{k}_{2} & \ldots & \hat{k}_{M} & \tilde{k}_{1} & \tilde{k}_{2} & \ldots & \tilde{k}_{M}
\end{array}\right]^{T}, \\
\boldsymbol{b}=\left[\begin{array}{lllll}
y\left(t_{1}\right) & y\left(t_{2}\right) & \ldots & y\left(t_{n}\right)
\end{array}\right]^{T}
\end{gathered}
$$

Next, the zeros are determined using (2.30) and (2.31). The zeros are taken as the new set of starting poles in another iteration of the above formulation. This process is repeated until the desired convergence criteria is obtained.

\section{Stage 2 : Residue Identification}

In this stage, the residues of (2.45) are determined using the final set of poles from stage 1. These poles are taken as the poles of the transfer function, $H(s)$, in $(2.45)$. Then, using (2.48), the output response becomes

$$
Y(s)=d X(s)+\sum_{m=1}^{M} \frac{k_{m} X(s)}{s-p_{m}}
$$

In the time-domain this is written as

$$
y(t)=d x(t)+\sum_{m=1}^{M} k_{m} x_{m}(t)
$$

where $x_{m}(t)$ is defined in (2.51). Next, the unknown matrix values $d$ and $\left\{k_{m}\right\}$ for $m=1,2, \ldots, M$ are found using a least squares formulation, $\mathbf{A} \boldsymbol{x}=\boldsymbol{b}$, with the 
matrices defined as

$$
\underbrace{\left[\begin{array}{ccccc}
x\left(t_{1}\right) & x_{1}\left(t_{1}\right) & x_{2}\left(t_{1}\right) & \ldots & x_{M}\left(t_{1}\right) \\
x\left(t_{2}\right) & x_{1}\left(t_{2}\right) & x_{2}\left(t_{2}\right) & \ldots & x_{M}\left(t_{2}\right) \\
\vdots & \vdots & \vdots & \ddots & \vdots \\
x\left(t_{n}\right) & x_{1}\left(t_{n}\right) & x_{2}\left(t_{n}\right) & \ldots & x_{M}\left(t_{n}\right)
\end{array}\right]}_{\mathbf{A}} \underbrace{\left[\begin{array}{c}
d \\
k_{1} \\
k_{2} \\
\ldots \\
k_{M}
\end{array}\right]}_{\boldsymbol{x}}=\underbrace{\left[\begin{array}{c}
y\left(t_{1}\right) \\
y\left(t_{2}\right) \\
\ldots \\
y\left(t_{n}\right)
\end{array}\right]}_{\boldsymbol{b}} .
$$

\section{Modifications for Complex Poles}

For complex poles, modifications to the least squares problems defined by (2.55) (2.56) and (2.59) must be made. If each of the partial fraction terms $m$ and $m+1$ in (2.45) correspond to a complex pair, then we have $\frac{M}{2}$ complex pairs in total. Define the complex pole-residue pairs as

$$
\begin{aligned}
p_{m} & =\mathfrak{R e}\left(p_{m}\right)+j \mathfrak{I m}\left(p_{m}\right), & p_{m+1} & =\mathfrak{R e}\left(p_{m}\right)-j \mathfrak{I m}\left(p_{m}\right), \\
k_{m} & =\mathfrak{R e}\left(k_{m}\right)+j \mathfrak{I m}\left(k_{m}\right), & k_{m+1} & =\mathfrak{R e}\left(k_{m}\right)-j \mathfrak{I m}\left(k_{m}\right) .
\end{aligned}
$$

Next, define the complex-pole convolution pairs as

$$
\begin{array}{rlrl}
x_{m}(t) & =\mathfrak{R e}\left(x_{m}(t)\right)+j \mathfrak{I m}\left(x_{m}(t)\right), & & x_{m+1}(t)=\mathfrak{R e}\left(x_{m}(t)\right)-j \mathfrak{I m}\left(x_{m}(t)\right), \\
y_{m}(t)=\mathfrak{R e}\left(y_{m}(t)\right)+j \mathfrak{I m}\left(y_{m}(t)\right), & y_{m+1}(t)=\mathfrak{R e}\left(y_{m}(t)\right)-j \mathfrak{I m}\left(y_{m}(t)\right),
\end{array}
$$


and the real and complex terms

$$
\begin{array}{ll}
\hat{x}_{m}(t)=x_{m}(t)+x_{m}^{*}(t), & \hat{x}_{m}^{\prime}(t)=j x_{m}(t)-j x_{m}^{*}(t), \\
\hat{y}_{m}(t)=y_{m}(t)+y_{m}^{*}(t), & \hat{y}_{m}^{\prime}(t)=j y_{m}(t)-j y_{m}^{*}(t) .
\end{array}
$$

The definitions in (2.55) and (2.56) are modified to yield

$$
\mathbf{A}=\left[\begin{array}{ll}
\mathbf{A}_{1} & \mathbf{A}_{2}
\end{array}\right], \quad \boldsymbol{x}=\left[\begin{array}{ll}
\boldsymbol{x}_{1} & \tilde{\boldsymbol{k}}
\end{array}\right]^{T}, \quad \boldsymbol{b}=\left[\begin{array}{llll}
y\left(t_{1}\right) & y\left(t_{2}\right) & \ldots & y\left(t_{n}\right)
\end{array}\right]^{T}
$$

with

$$
\begin{aligned}
& \mathbf{A}_{\mathbf{1}}=\left[\begin{array}{cccccccc}
x\left(t_{1}\right) & \hat{x}_{1}\left(t_{1}\right) & \hat{x}_{1}^{\prime}\left(t_{1}\right) & \hat{x}_{2}\left(t_{1}\right) & \hat{x}_{2}^{\prime}\left(t_{1}\right) & \ldots & \hat{x}_{\frac{M}{2}}\left(t_{1}\right) & \hat{x}_{\frac{M}{2}}^{\prime}\left(t_{1}\right) \\
x\left(t_{2}\right) & \hat{x}_{1}\left(t_{2}\right) & \hat{x}_{1}^{\prime}\left(t_{2}\right) & \hat{x}_{2}\left(t_{2}\right) & \hat{x}_{2}^{\prime}\left(t_{2}\right) & \ldots & \hat{x}_{\frac{M}{2}}\left(t_{2}\right) & \hat{x}_{\frac{M}{2}}^{\prime}\left(t_{2}\right) \\
\vdots & \vdots & \vdots & \vdots & \vdots & \ddots & \vdots & \vdots \\
x\left(t_{n}\right) & \hat{x}_{1}\left(t_{n}\right) & \hat{x}_{1}^{\prime}\left(t_{n}\right) & \hat{x}_{2}\left(t_{n}\right) & \hat{x}_{2}^{\prime}\left(t_{n}\right) & \ldots & \hat{x}_{\frac{M}{2}}\left(t_{n}\right) & \hat{x}_{\frac{M}{2}}^{\prime}\left(t_{n}\right)
\end{array}\right], \\
& \mathbf{A}_{2}=\left[\begin{array}{ccccccc}
-\hat{y}_{1}\left(t_{1}\right) & -\hat{y}_{1}^{\prime}\left(t_{1}\right) & -\hat{y}_{2}\left(t_{1}\right) & -\hat{y}_{2}^{\prime}\left(t_{1}\right) & \ldots & -\hat{y}_{\frac{M}{2}}\left(t_{1}\right) & -\hat{y}_{\frac{M}{2}}^{\prime}\left(t_{1}\right) \\
-\hat{y}_{1}\left(t_{2}\right) & -\hat{y}_{1}^{\prime}\left(t_{2}\right) & -\hat{y}_{2}\left(t_{2}\right) & -\hat{y}_{2}^{\prime}\left(t_{2}\right) & \ldots & -\hat{y}_{\frac{M}{2}}\left(t_{2}\right) & -\hat{y}_{\frac{M}{2}}^{\prime}\left(t_{2}\right) \\
\ldots & \ldots & \ldots & \ldots & \ddots & \ldots & \ldots \\
-\hat{y}_{1}\left(t_{n}\right) & -\hat{y}_{1}^{\prime}\left(t_{n}\right) & -\hat{y}_{2}\left(t_{n}\right) & -\hat{y}_{2}^{\prime}\left(t_{n}\right) & \ldots & -\hat{y}_{\frac{M}{2}}\left(t_{n}\right) & -\hat{y}_{\frac{M}{2}}^{\prime}\left(t_{n}\right)
\end{array}\right],
\end{aligned}
$$

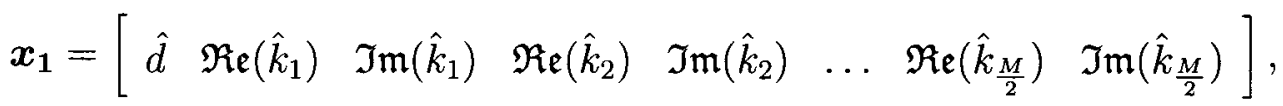

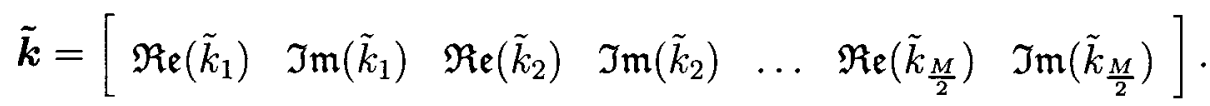


Similarly, the variables in the least squares problem, $\mathbf{A} \boldsymbol{x}=\boldsymbol{b}$, from (2.59) become

$$
\begin{gathered}
\mathbf{A}=\left[\begin{array}{cccccccc}
x\left(t_{1}\right) & \hat{x}_{1}\left(t_{1}\right) & \hat{x}_{1}^{\prime}\left(t_{1}\right) & \hat{x}_{2}\left(t_{1}\right) & \hat{x}_{2}^{\prime}\left(t_{1}\right) & \ldots & \hat{x}_{\frac{M}{2}}\left(t_{1}\right) & \hat{x}_{\frac{M}{2}}^{\prime}\left(t_{1}\right) \\
x\left(t_{2}\right) & \hat{x}_{1}\left(t_{2}\right) & \hat{x}_{1}^{\prime}\left(t_{2}\right) & \hat{x}_{2}\left(t_{2}\right) & \hat{x}_{2}^{\prime}\left(t_{2}\right) & \ldots & \hat{x}_{\frac{M}{2}}\left(t_{2}\right) & \hat{x}_{\frac{M}{2}}^{\prime}\left(t_{2}\right) \\
\vdots & \vdots & \vdots & \vdots & \vdots & \ddots & \vdots & \vdots \\
x\left(t_{n}\right) & \hat{x}_{1}\left(t_{n}\right) & \hat{x}_{1}^{\prime}\left(t_{n}\right) & \hat{x}_{2}\left(t_{n}\right) & \hat{x}_{2}^{\prime}\left(t_{n}\right) & \ldots & \hat{x}_{\frac{M}{2}}\left(t_{n}\right) & \hat{x}_{\frac{M}{2}}^{\prime}\left(t_{n}\right)
\end{array}\right]^{T} \\
\boldsymbol{x}=\left[\begin{array}{llllllll}
d & \mathfrak{R e}\left(k_{1}\right) & \mathfrak{I m}\left(k_{1}\right) & \mathfrak{R e}\left(k_{2}\right) & \mathfrak{I m}\left(k_{2}\right) & \ldots & \mathfrak{R e}\left(k_{\frac{M}{2}}\right) & \mathfrak{I m}\left(k_{\frac{M}{2}}\right)
\end{array}\right]^{T}, \\
\boldsymbol{b}=\left[\begin{array}{llllll}
y\left(t_{1}\right) & y\left(t_{2}\right) & \ldots & y\left(t_{n}\right)
\end{array}\right]^{T} .
\end{gathered}
$$

The resulting overdetermined least squares problems of the form $\mathbf{A} \boldsymbol{x}=\boldsymbol{b}$ are formulated in terms of real quantities to preserve complex conjugate pairings. This is written as

$$
\underbrace{\left[\begin{array}{c}
\mathfrak{R e}(\mathbf{A}) \\
\mathfrak{I m}(\mathbf{A})
\end{array}\right]}_{\hat{\mathbf{A}}} \boldsymbol{x}=\underbrace{\left[\begin{array}{c}
\mathfrak{R e}(\boldsymbol{b}) \\
\mathfrak{I m}(\boldsymbol{b})
\end{array}\right]}_{\hat{\boldsymbol{b}}}
$$

or

$$
\hat{\mathbf{A}} \boldsymbol{x}=\hat{\boldsymbol{b}},
$$

which can be solved using the standard Householder algorithm.

The zeros of $\sigma(s)$ are found using (2.42) and (2.43) for complex pole pairs, analogous to the FD-VF algorithm.

\subsubsection{Prony's Algorithm}

Prony's algorithm is applied directly to a set of time-domain data, $\{y(t)\}$, to produce an approximation for this data as a sum of exponentials in the time-domain. The 
approximation is of the form [56]

$$
y_{a}(t)=\sum_{m=1}^{M} k_{m} e^{p_{m} t}
$$

where $\left\{p_{m}\right\}$ and $\left\{k_{m}\right\}$ are the poles and residues, respectively, of the Laplace-domain representation for $y_{a}(t)$ as a rational function. Let $T$ be the sample period, $N$ be the number of time points, and

$$
y(i T)=y_{i}, \quad i=0,1,2, \ldots, N-1
$$

The objective of Prony's algorithm is to determine the $2 M$ variables $\left\{k_{m}, p_{m}\right\}$ in the function $y_{a}(t)$, which best approximate the data. To begin, define the new variables $\left\{z_{m}\right\}$ as

$$
z_{m}=e^{p_{m} T}, \quad m=1,2, \ldots, M,
$$

and the related variables $\left\{\alpha_{i}\right\}$ by

$$
\sum_{i=0}^{M} \alpha_{i} z^{i}=\prod_{m=1}^{M}\left(z-z_{m}\right), \quad \alpha_{M}=1 .
$$

Then, the requirement

$$
y_{a}(i T)=y_{i}, \quad i=0,1,2, \ldots, N-1
$$

is used with (2.71) - (2.73) to obtain

$$
y_{i}=\sum_{m=1}^{M} k_{m} z_{m}^{i}, \quad i=0,1,2, \ldots, N-1
$$


Equations (2.74) and (2.76) are combined to form

$$
\sum_{i=0}^{M} y_{k+i} \alpha_{i}=\sum_{i=0}^{M}\left\{\sum_{m=1}^{M} k_{m} z_{m}^{k+i}\right\} \alpha_{i}=\sum_{m=1}^{M} k_{m} z_{m}^{k}\left\{\sum_{i=0}^{M} \alpha_{i} z^{i}\right\}=0
$$

for $k=0,1, \ldots, N-1-M$. This yields a linear system of equations

$$
\sum_{i=0}^{M-1} y_{k+i} \alpha_{i}=-y_{k+M}, \quad k=0,1, \ldots, N-1-M
$$

which is solved as an overdetermined least squares problem for the variables $\left\{\alpha_{i}\right\}$. The values $\left\{z_{m}\right\}$ are determined using (2.74) and subsequently, the poles are found with

$$
p_{m}=\frac{1}{T} \ln \left(z_{m}\right), \quad m=1,2, \ldots, M
$$

Next, the residues, $\left\{k_{m}\right\}$ are found solving (2.76) as an overdetermined least squares problem for $i=0,1, \ldots, N-1$.

\subsection{Macromodeling Techniques for Long-Delay Networks}

As discussed in chapter 1 , increasing operating frequencies combined with long delay networks result in high-order rational function approximations and subsequently, prohibitively CPU expensive SPICE simulations. The benefits of using delay extraction to reduce the size of long delay interconnect macromodels [7], [17] has initiated attempts to construct a delay extraction based macromodel for networks characterized by tabulated data. There have been a handful of papers discussing this 
topic [23]-[25], [27]-[29], and these methods are reviewed in this section. First, Hilbert transform based delay estimation is reviewed [23]-[26]. Second, optimization based delay estimation vector fitting algorithms are discussed [27], [28]. Third, the Gabor transform based macromodeling technique developed for networks characterized by tabulated frequency-domain data [29] is explained.

\subsubsection{Delay based Macromodeling via Hilbert Transforms}

In order to extract a delay from the transfer function, it is important to obtain an accurate method to estimate this delay. It has been shown that using the properties of minimum phase for passive structures, the port-to-port delay can be estimated using the Hilbert transform [23]-[26]. A function, $H(s)$, is a minimum phase function if all its poles and zeros lie in the LHP. In multi-port stable networks, this property is only exhibited by the self-responses, or diagonal elements of the system.

Consider the two-port network with a transfer function given by,

$$
\mathbf{H}(s)=\left[\begin{array}{ll}
H_{11}(s) & H_{12}(s) \\
H_{21}(s) & H_{22}(s)
\end{array}\right]
$$

The off diagonal elements, which represent port-to-port functionality of the system, are not minimum phase functions, unlike the self response $H_{11}(s)$ and $H_{22}(s)$. Using the fact that any stable system function can be written as a function of a product of a minimum phase function and an all-pass function, $H_{12}(s)$ is written as

$$
H_{12}(s)=H_{\min }(s) H_{A P}(s),
$$


where $H_{\min }(s)$ is the minimum phase function and $H_{A P}$ is the all-pass function. An all-pass function is defined as having a magnitude of 1 over the entire frequency range. Next, if $\tau$ is the delay between port 1 and 2 in the system, then $H_{12}(s)$ can also be written as

$$
H_{12}(s)=H_{12}^{\prime}(s) e^{-s \tau}
$$

Note that $e^{-s \tau}$ has magnitude of unity over the entire frequency range, which makes it equivalent to the all-pass function, leaving $H_{12}^{\prime}(s)$ as the minimum phase function.

It is known that the magnitude and the phase of a minimum phase function are related by the Hilbert transform. This property is used to obtain an estimate of the delay. The process begins using the relation

$$
\left|H_{12}^{\prime}(j \omega)\right|=\left|H_{\min }(j \omega)\right|=\left|H_{12}(j \omega)\right|
$$

Then, using the Hilbert transform, the phase can be calculated as

$$
\arg \left[H_{\min }(j \omega)\right]=-\frac{1}{2 \pi} P \int_{-\pi}^{\pi} \log \left|H_{\min }(j \omega)\right| \cot \left(\frac{\omega-\theta}{2}\right) d \theta
$$

where $P$ represents the Cauchy Principal value. Combining (2.82) and (2.83) yields

$$
H_{A P}(j \omega)=\frac{H_{12}(j \omega)}{H_{\min }(j \omega)}=e^{-j \omega \tau}
$$

which can be rearranged to obtain an expression for the delay as

$$
\tau=-\frac{\arg \left(H_{A P}(j \omega)\right)}{\omega}
$$


The final formula for the delay is obtained as

$$
\tau=-\frac{\arg \left(\frac{H_{12}(j \omega)}{\left|H_{12}(j \omega)\right| e^{\frac{j}{2 \pi} P \int_{-\pi}^{\pi} \log \left|H_{12}(j \omega)\right| \cot \left(\frac{\omega-\theta}{2}\right) d \theta}}\right)}{\omega}
$$

Once the delay has been estimated, transient simulation is carried out using signal flow graph techniques [26].

\subsubsection{Delay based Vector Fitting using Optimization}

In [27], [28], delay estimation is incorporated into the FD-VF algorithm via optimization. These techniques are given below.

\section{Direct Delay Extraction using Rational Approximations}

It is shown in [28], that extracting the lossless propagation delay from a system may not lead to an optimal rational fitting for the remaining data (once the delay has been extracted). That is, the rms-error in the fitting could be reduced if a slightly larger or smaller delay is extracted. Therefore, in [28] the vector fitting algorithm is applied to the function

$$
H(s) e^{s \tau}=\sum_{m=1}^{M} \frac{R_{m}}{s-p_{m}}
$$

to obtain a rational approximation, using an initial estimate for the delay, $\tau$. Next, the rms-error for the fitting is calculated. This process is repeated with an optimization algorithm to obtain the value, $\tau$, which leads to the smallest rms-error. 


\section{Delay Extraction using Magnitude Squared Approximations}

The above methodology was modified in [27]. Consider the rational minimum phase shift function, $f(s)$, having $N$ poles and $N-1$ zeros in the LHP. Next, note that the magnitude squared, $|f(s)|^{2}$ of the function has zero phase angle, $2 N$ poles, and $2 N-2$ zeros, which include the $N$ poles and $N-1$ zeros of $f(s)$ and their symmetrical counterparts in the RHP. If the magnitude square response is fitted with a rational function, then discarding the zeros and poles having a positive real part gives a minimum phase function, $f_{\text {rat }}(s)$. If FD-VF is used to fit $|f(s)|^{2}$, then the magnitude of $f(s)$ matches that of $f_{\text {rat }}(s)$ in the least squares sense. Assume both $\mathfrak{R e}\left\{z_{m}\right\}<0$ and $\mathfrak{R e}\left\{p_{m}\right\}<0$, then write

$$
|f(s)|^{2} \cong k \frac{\prod_{m=1}^{M-1}\left(s-z_{m}\right)\left(s+z_{m}\right)}{\prod_{m=1}^{M}\left(s-p_{m}\right)\left(s+p_{m}\right)}, \quad f_{r a t}(s)=\sqrt{(k)} \frac{\prod_{m=1}^{M-1}\left(s-z_{m}\right)}{\prod_{m=1}^{M}\left(s-p_{m}\right)}
$$

Once these functions have been obtained, the optimal time-delay of the system is found using an optimization algorithm, which searches for the delay, $\tau$, that minimizes the rms-error in the approximation (this avoids manual frequency sweeping).

Conventional FD-VF does not obtain poles and residues that are symmetrical w.r.t the imaginary axis. Therefore, some modifications are made to the FD-VF algorithm. To begin, we consider the least squares problem

$$
\left(|f|^{2}|\sigma|^{2}\right)_{f i t}(s)-|f(s)|^{2}|\sigma(s)|_{f i t}^{2} \approx 0
$$


Then, the functions in (2.90) are defined as

$$
\begin{array}{r}
\left(|f|^{2}|\sigma|^{2}\right)_{f i t}(s)=\sum_{m=1}^{M} c_{m}\left(\frac{1}{s-p_{m}}-\frac{1}{s+p_{m}}\right) \\
|\sigma(s)|_{f i t}^{2}=\sum_{m=1}^{M} \bar{c}_{m}\left(\frac{1}{s-p_{m}}-\frac{1}{s+p_{m}}\right)+\bar{d}
\end{array}
$$

where $\left\{c_{m}\right\},\left\{\bar{c}_{m}\right\}, \bar{d}$ are unknown, and $\left\{p_{m}\right\}$ are known starting poles [30]. Next, (2.90) is formulated as a least squares problem in the form $\mathbf{A x}=\boldsymbol{b}$. Define the terms

$$
\begin{gathered}
g_{k, m}=\frac{1}{s_{k}-p_{m}}-\frac{1}{s_{k}+p_{m}} \\
g_{k, m}=\frac{1}{s_{k}-p_{m}}-\frac{1}{s_{k}+p_{m}}+\frac{1}{s_{k}-p_{m}^{*}}-\frac{1}{s_{k}+p_{m}^{*}}, \\
g_{k, m+1}=\frac{j}{s_{k}-p_{m}}-\frac{j}{s_{k}+p_{m}}+\frac{j}{s_{k}-p_{m}^{*}}-\frac{j}{s_{k}+p_{m}^{*}}
\end{gathered}
$$

where (2.93a) is used for real poles and (2.93b) is used for complex pole-pairs. The $k^{\text {th }}$ row of $\mathbf{A}$, where $k=1,2, \ldots, N_{s}$ and $N_{s}$ is the number of frequency samples, is defined as

$$
\mathbf{A}_{\mathbf{k}}=\left[\begin{array}{ll}
\mathbf{A}_{\mathbf{k}, 1} & \mathbf{A}_{\mathbf{k}, 2}
\end{array}\right]
$$

with

$$
\begin{aligned}
& \mathbf{A}_{\mathbf{k}, \mathbf{1}}=\left[\begin{array}{lll}
g_{k, 1} & \ldots & g_{k, M}
\end{array}\right] \\
& \mathbf{A}_{\mathbf{k}, \mathbf{2}}=-\left|f\left(s_{k}\right)\right|^{2}\left[\begin{array}{llll}
g_{k, 1} & \ldots & g_{k, M} & 1
\end{array}\right], \\
& \boldsymbol{x}=\left[\begin{array}{lllllll}
c_{1} & \ldots & c_{M} & \bar{c}_{1} & \ldots & \bar{c}_{M} & \bar{d}
\end{array}\right]^{T} .
\end{aligned}
$$

If (2.90) is solved as is, then $\boldsymbol{b}=\mathbf{0}$, and the least squares problem of $\mathbf{A} \boldsymbol{x}=\boldsymbol{b}$ yields a 
null solution. In order to avoid this, an additional constraint is added to the problem to give a non-zero vector, $\boldsymbol{b}$. This adds an additional row to the matrix $\mathbf{A}$ and $\boldsymbol{b}$ such that

$$
\mathbf{A}_{\mathbf{k}+\mathbf{1}}=\left[\begin{array}{llll}
\sum_{k=1}^{N_{s}} g_{k, 1} & \ldots & \sum_{k=1}^{N_{s}} g_{k, M} & N_{s} \bar{d}
\end{array}\right], \quad \boldsymbol{b}=\left[\begin{array}{llll}
0 & \ldots & 0 & N_{s}
\end{array}\right]^{T}
$$

This is equivalent to adding the constraint

$$
\sum_{k=1}^{N_{s}}\left(\sum_{m=1}^{M} g_{k, m} \bar{c}_{m}+\bar{d}\right)=N_{s}
$$

Next, the zeros of $\sigma_{f i t}^{2}(s)$ are calculated. Equation (2.92) is written as

$$
|\sigma|_{f i t}^{2}(s)=\sum_{m=1}^{M} \frac{2 \bar{c}_{m} p_{m}}{s^{2}-p_{m}^{2}}+\bar{d}=\bar{d} \frac{\prod_{m=1}^{M}\left(s^{2}-\bar{z}_{m}^{2}\right)}{\prod_{m=1}^{M}\left(s^{2}-p_{m}^{2}\right)}
$$

Using (2.98), the values $\left\{\bar{z}_{m}^{2}\right\}$ are found as in [30], whose square roots, $\left\{\bar{z}_{m}\right\}$, are the poles, $\left\{\bar{p}_{m}\right\}$, chosen to begin the next iteration in the algorithm (see section 2.5.1 for details). If any negative $\left\{z_{m}^{2}\right\}$ occur during this process, their signs are changed before extracting their square roots [28], [30].

\subsubsection{Delay based Macromodeling via Gabor Transforms}

Following is a review of the Gabor transform based macromodeling technique for networks characterized by tabulated frequency-domain data [29]. First, a review of the Gabor transform is given. Next, the macromodeling techniques for constructing 
delayed rational function approximations using the Gabor transform are presented.

\section{Time-Frequency Decomposition}

Consider a generic scalar transfer function $H(\omega)$. The Gabor transform of this function is defined as [29], [59]

$$
\mathcal{G}=\int_{-\infty}^{+\infty} H(\varepsilon) W_{\omega, \tau}^{*}(\varepsilon) d \varepsilon, \quad W_{\omega, \tau}(\varepsilon)=W(\varepsilon-\omega) e^{-j \epsilon \tau}, \quad W(\varepsilon)=\pi^{1 / 4} e^{\varepsilon^{2} / 2}
$$

where $\tau$ represents time or time-delay in the system. The function $\mathcal{G}(\omega, \tau)$ can be considered the inverse Fourier transform of $H(\varepsilon)$, but retaining only those frequency components located in a frequency band about $\omega$. The inversion formula for this transform is

$$
H(\varepsilon)=\frac{1}{2 \pi} \int_{-\infty}^{+\infty} \int_{-\infty}^{+\infty} \mathcal{G}(\omega, \tau) W_{\omega, \tau}(\varepsilon) d \omega d \tau
$$

which allows for the recovery of the original function from its coefficients. An important property of the Gabor transform is that the reconstruction formula in (2.100) can be combined with the time-frequency localization process in order to split $H(\varepsilon)$ into separate components such that

$$
H(\varepsilon)=\sum_{k=1}^{K} H_{k}(\varepsilon), \quad H_{k}(\varepsilon)=\frac{1}{2 \pi} \iint_{\Omega_{k}} \mathcal{G}(\omega, \tau) W_{\omega, \tau}(\varepsilon) d \omega d \tau, \quad \bigcup_{k=1}^{K} \Omega_{k}=\mathfrak{R}^{2}
$$

The entire time-frequency plane is separated into disjoint subsets, $\Omega_{k}$, the superposition of which leads to the original function $H(\varepsilon)$. 


\section{Separation and Approximation of Single-Delay Atoms}

In order to separate the transfer function, $H(\varepsilon)$, it is necessary to determine the appropriate subsets $\Omega_{k}$. This is accomplished by averaging the time-frequency energy distribution using the relation

$$
\xi(\tau)=\frac{1}{2 \pi} \int_{-\infty}^{+\infty} \mathcal{G}(\omega, \tau)^{2} d \omega
$$

and taking the well-pronounced maxima, $\tilde{T}_{k}$, as the approximate delays in the system. The minima between each pair of maxima, $\tau_{k}$, are used to separate the single delay atoms. The subsets, $\Omega_{k}$, are defined as

$$
\Omega_{k}=\left\{(\omega, \tau): \tau \in\left(\tau_{k}, \tau_{k+1}\right), \forall \omega\right\}
$$

which are used in (2.101) to obtain each set of data $\left\{H_{k}(\varepsilon)\right\}$. Then, FD-VF with rms delay optimization [27], [28] (as described in section (2.6.2)) is applied to each of these data sets, $\left\{H_{k}(\varepsilon)\right\}$, to obtain

$$
H(\varepsilon)=\sum_{k=1}^{K} H_{k}^{\prime}(\varepsilon) e^{-j \varepsilon \hat{T}_{k}}=\sum_{k=1}^{K} \sum_{m=1}^{M} \frac{R_{m, k}}{j \varepsilon-p_{m, k}} e^{-j \varepsilon \hat{T}_{k}}
$$

where for the $k^{\text {th }}$ segment, $\hat{T}_{k}$ is the final delay chosen from the optimization process, $M_{k}$ is the number of poles, $\left\{R_{m, k}\right\}$ are the residues, and $\left\{p_{m, k}\right\}$ are the poles. 


\section{Chapter 3}

\section{Passive Macromodeling of \\ Transmission Line Type}

\section{Interconnects Characterized by}

\section{Tabulated Data}

\subsection{Introduction}

Signal integrity analysis of transmission lines has developed significantly over the past few years [1], [3], [7], [9]-[17] due to the demand for faster and more accurate highspeed interconnect models. Research in this area has been primarily focused on the construction of macromodels given the tabulated p.u.l transmission line parameters. However, the p.u.l parameters are not always given, and in many cases, only the tabulated S, Y, H, or Z parameters are known. In such cases, the data is fit with a 
rational function approximation using the FD-VF algorithm [30] or equivalent, and converted into a circuit for SPICE simulation. However, for long delay lines, this yields a high-order rational function approximation leading to a large and slow circuits for SPICE simulation. In order to overcome this problem, the delay extraction-based passive compact transmission line (DEPACT) macromodeling algorithm [7], [9] is extended in this chapter to handle tabulated $\mathrm{S}, \mathrm{Y}, \mathrm{Z}$, or $\mathrm{H}$ parameters.

This chapter is organized as follows. Section 3.2 describes the problem formulation. Section 3.3 describes a method to obtain a unique logarithm; the resulting logarithm can be fit more easily than using standard principal value of the logarithm. Section 3.4 outlines the macromodel formulation and is followed by section 3.5, which describes the methods used to realize the macromodel in standard SPICE-like simulators. Finally, section 3.6 presents some numerical results.

\subsection{Problem Formulation}

A single set of transmission lines is known to satisfy Telegraphers equations [1]. It is shown in section 2.2 that the solution to this system in $\mathrm{H}$ parameter form is given by

$$
\left[\begin{array}{c}
\mathbf{V}(d, s) \\
\mathbf{I}(d, s)
\end{array}\right]=\left[\begin{array}{ll}
\mathbf{H}_{11}(s) & \mathbf{H}_{12}(s) \\
\mathbf{H}_{21}(s) & \mathbf{H}_{22}(s)
\end{array}\right]\left[\begin{array}{c}
\mathbf{V}(0, s) \\
\mathbf{I}(0, s)
\end{array}\right]=e^{\mathbf{\Phi}(s)}\left[\begin{array}{c}
\mathbf{V}(0, s) \\
\mathbf{I}(0, s)
\end{array}\right]
$$

The function $\boldsymbol{\Phi}(s)$ can be obtained by calculating the matrix logarithm of the H parameters. Once the function $\boldsymbol{\Phi}(s)$ is known, the DEPACT macromodeling methodology can be applied to the system. While the calculation of the matrix logarithm may seem trivial, the solution is not unique. 


\subsection{A Unique Matrix Logarithm}

The logarithm of a matrix has an infinite number of possible values. This can be seen by considering a two-conductor example. Let $\mathbf{V}(s)$ and $\mathbf{D}(s)$ be the matrices containing the eigenvectors and eigenvalues, respectively, for $\boldsymbol{\Phi}(s)$. Then,

$$
\log \left(e^{[\mathbf{\Phi}(s)]}\right)=\log \left(e^{[\mathbf{V}(s)][\mathbf{D}(s)]\left[\mathbf{V}^{-\mathbf{1}}(s)\right]}\right)=[\mathbf{V}(s)] \log \left(e^{[\mathbf{D}(s)]}\right)\left[\mathbf{V}^{-\mathbf{1}}(s)\right]
$$

Evaluating the remaining logarithm of the function gives

$$
\begin{aligned}
\log \left(e^{[\mathbf{D}(s)]}\right) & =\left[\begin{array}{cc}
\log \left(e^{D_{11}(s) \pm 2 j \pi p_{1}}\right) & 0 \\
0 & \log \left(e^{D_{22}(s) \pm 2 j \pi p_{2}}\right)
\end{array}\right] \\
& =\left[\begin{array}{cc}
D_{11}(s) \pm 2 j \pi p_{1} & 0 \\
0 & D_{22}(s) \pm 2 j \pi p_{2}
\end{array}\right]
\end{aligned}
$$

where $p_{1}$ and $p_{2}$ are integers from $-\infty$ to $\infty$. Algorithms for calculating the logarithm of a matrix calculate the principal value [60], which is the value such that the phase of all the eigenvalues lies between $-\pi$ and $\pi$. In this example, $D_{11}(s)$ and $D_{22}(s)$ are the principal values. Then, the possibilities for the matrix logarithm of the entire function, $\Phi(s)$, are

$$
\mathbf{\Phi}(s)=[\mathbf{V}(s)]\left[\begin{array}{cc}
D_{11}(s) & 0 \\
0 & D_{22}(s)
\end{array}\right]\left[\mathbf{V}(s)^{-1}\right]+2 j \pi[\mathbf{V}(s)]\left[\begin{array}{cc} 
\pm p_{1} & 0 \\
0 & \pm p_{2}
\end{array}\right]\left[\mathbf{V}(s)^{-1}\right]
$$

All values of $p_{1}$ and $p_{2}$ lead to a logarithm that would satisfy the system. However, in the case of measured/tabulated data, the purpose is to determine the values of $p_{1}$ and 
$p_{2}$ resulting in a data sequence that can be most easily fit with a rational function approximation. Consider the logarithm of the $\mathrm{H}$ parameters for an ideal transmission line. It is clear from Fig. 3.1 that the principal value is significantly more difficult to fit than the proposed algorithm.

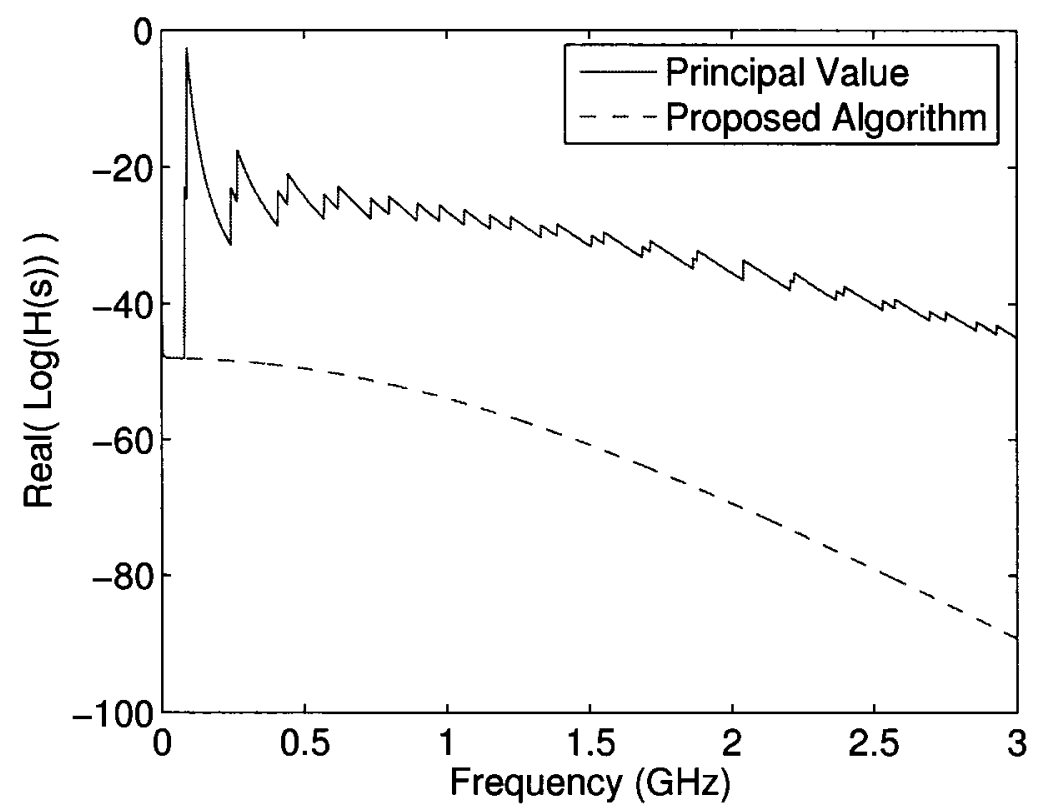

Fig. 3.1: A comparison of the matrix logarithm computation

Therefore, the goal is to choose the values of $p_{1}$ and $p_{2}$ that minimize the change in the function between each frequency point, yielding the data set that can most easily be fit with a rational function approximation. Consider an $m$-port system. Let $\Phi^{i}(s)$ be the matrix logarithm of the $i^{\text {th }}$ frequency point and let $p_{n}^{i}$ be the phase integers of the eigenvalues at this frequency point, where $n=1,2, \ldots, m$. Then, a least squares formulation is developed in which $p_{n}^{i-1}$ are the known values at the previous frequency point, and the values of $p_{n}^{i}$ are unknown. The best choice for each $p_{n}^{i}$ is the one which produces the smoothest function $\Phi(s)$. Therefore, it is desired to minimize the least 
squares problem

$$
\psi(s)=\sum_{k=1}^{m} \sum_{l=1}^{m}\left[\operatorname{real}\left(\Phi_{k, l}^{i}-\Phi_{k, l}^{i-1}\right)^{2}+i m a g\left(\Phi_{k, l}^{i}-\Phi_{k, l}^{i-1}\right)^{2}\right]
$$

w.r.t the $p_{n}^{i}$, where $\Phi_{k, l}^{i}$ refers to the element $(k, l)$ in matrix $\boldsymbol{\Phi}(s)$. The values of $p_{n}^{0}$ for $n=1, \ldots, m$ are taken as 0 at $s=0$. This problem can be solved using a combinatorial optimization algorithm [61]. An exhaustive search method could also be used for systems with a small number of ports.

\subsection{Formulation of Hybrid Model}

Once the logarithm has been calculated, the function $\Phi(s)$ is fit with a low-order rational function approximation using FD-VF [30]. Passivity of this function is enforced using a passivity correction algorithm [39], [40]. This ensures the matrix function is positive definite. Following this, $\boldsymbol{\Phi}(s)$, is broken into

$$
\boldsymbol{\Phi}(s)=\boldsymbol{\Phi}_{R}(s)+s \boldsymbol{\Phi}_{I}(s) .
$$

Next, the methodology of DEPACT is applied to this system. Let

$$
\Phi_{a}(s)=\Phi_{I}(s)-\Phi_{I}(\infty)
$$


where $\boldsymbol{\Phi}_{a}(s)$ is a positive definite matrix. Then, the MLF of (2.17) is applied such that

$$
\begin{aligned}
e^{\Phi(s)} & =e^{s \Phi_{I}(\infty)+s \Phi_{a}(s)+\Phi_{R}(s)} \\
& \cong \prod_{k=1}^{m} \Psi_{k}, \quad \Psi_{k}=e^{\frac{s \Phi_{I}(\infty)}{2 m}} e^{\frac{\Phi_{R}(s)+s \Phi_{a}(s)}{m}} e^{\frac{s \Phi_{I}(\infty)}{2 m}}
\end{aligned}
$$

where $m$ is the number of segments used in the approximation. From (3.8), a set of equivalent subcircuits is constructed for implementation in conventional SPICE-like simulators.

\subsection{Realization of SPICE Macromodel}

In order to realize the proposed macromodel, the MLF relations must be converted into equivalent circuit representations [8]. To demonstrate this process, consider the network represented by (3.8). This equation can be modeled as a cascade of $m$ subnetworks representing each of the $\Psi_{k}$. Each of these subnetworks is then modeled as a cascade of lossy and lossless transmission lines. A model of $\Psi_{k}$ is shown in Fig. 3.2. The lossy terms can be macromodeled using the passive MRA techniques of section 2.2.2 [10]-[13]. Each of these MRA models requires a much lower order approximation than applying MRA directly on the full data set. The lossless sections are modeled using a modified $\mathrm{MoC}$ approach, which decouples the line equations. The resulting macromodel is passive by construction, provided the fitting of $\boldsymbol{\Phi}(s)$ is passive [7]. The remainder of this section outlines the realization of the lossless terms [7]. 


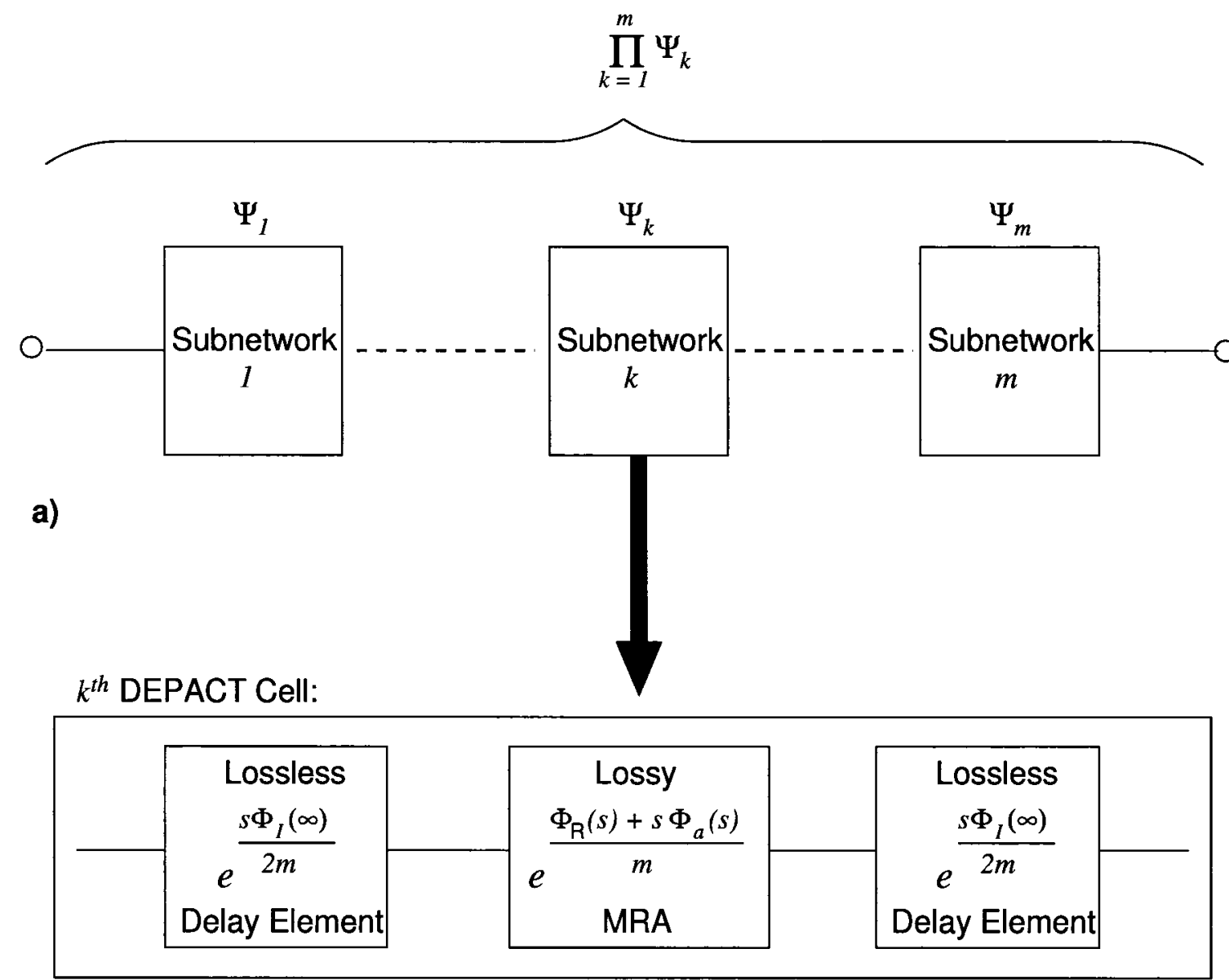

b)

Fig. 3.2: Macromodel realization of product terms in (3.8).

Using (3.7), define

$$
\left[\begin{array}{cc}
0 & -\mathbf{L}_{m} \\
-\mathbf{C}_{m} & 0
\end{array}\right]=\Phi_{I}(\infty) / 2 m .
$$

Then, each of the lossless terms, $e^{\frac{s \Phi_{I}(\infty)}{2 m}}$, is equivalent to the solution of Telegraphers 
equations for and $N$ port lossless transmission line of the form

$$
\begin{aligned}
\frac{\partial}{\partial x} \boldsymbol{v}(x, t) & =-\mathbf{L}_{m} \frac{\partial}{\partial t} \boldsymbol{i}(x, t) \\
\frac{\partial}{\partial x} \boldsymbol{i}(x, t) & =-\mathbf{C}_{m} \frac{\partial}{\partial t} \boldsymbol{v}(x, t)
\end{aligned}
$$

$\boldsymbol{v}(x, t)$ and $\boldsymbol{i}(x, t)$ are the terminal voltages and currents, respectively. This can be expressed in terms of the propagating modal voltages $\hat{\boldsymbol{v}}(x, t)$ and currents $\hat{\boldsymbol{i}}(x, t)[1]$

$$
\begin{gathered}
\boldsymbol{v}(x, t)=\mathbf{E}_{V} \hat{\boldsymbol{v}}(x, t), \\
\boldsymbol{i}(x, t)=\mathbf{E}_{I} \hat{\boldsymbol{i}}(x, t) .
\end{gathered}
$$

The transformation matrices $\mathbf{E}_{V}$ and $\mathbf{E}_{I}$ are found using the relations [1]

$$
\begin{aligned}
& \mathbf{E}_{I}=\mathbf{Q F S} \alpha \\
& \mathbf{E}_{V}=\mathbf{Q F}^{-1} \mathbf{S} \alpha^{-1}
\end{aligned}
$$

where $\mathbf{Q}$ is a matrix containing the right eigenvectors of $\mathbf{C}_{m}, \mathbf{F}^{2}$ is a diagonal matrix of the eigenvalues of $\mathbf{C}_{m}$, and $\mathbf{S}$ contains the right eigenvectors of the product $\mathrm{FQ}^{T} \mathbf{L}_{m} \mathrm{QF}$. The diagonal matrix $\alpha$ has its diagonal elements defined by

$$
\alpha_{i i}=\frac{1}{\sqrt{\sum_{k=1}^{N} E_{k i}^{2}}}
$$

with $\left\{E_{k i}\right\}$ as the elements of the matrix $\mathbf{E}=$ QFS. Then, the transformation 
matrices $\mathbf{E}_{I}$ and $\mathbf{E}_{V}$ are used to diagonalize $\mathbf{L}_{m}$ and $\mathbf{C}_{m}$ to obtain

$$
\begin{aligned}
& \hat{\mathbf{L}}=\mathbf{E}_{V}^{-1} \mathbf{L}_{m} \mathbf{E}_{I} \\
& \hat{\mathbf{C}}=\mathbf{E}_{I}^{-1} \mathbf{C}_{m} \mathbf{E}_{V},
\end{aligned}
$$

where $\hat{\mathbf{L}}$ and $\hat{\mathbf{C}}$ are diagonal matrices defined as $\hat{\mathbf{L}}=\operatorname{diag}\left\{\hat{l}_{1}, \hat{l}_{2}, \ldots, \hat{l}_{N}\right\}$ and $\hat{\mathbf{C}}=$ $\operatorname{diag}\left\{\hat{c}_{1}, \hat{c}_{2}, \ldots, \hat{c}_{N}\right\}$. Equation (3.10) can be written as a set of decoupled lossless lines using the modal parameters such that

$$
\begin{aligned}
\frac{\partial}{\partial x} \hat{\boldsymbol{v}}(x, t) & =-\hat{\mathbf{L}} \frac{\partial}{\partial t} \hat{\boldsymbol{i}}(x, t) \\
\frac{\partial}{\partial x} \hat{\boldsymbol{i}}(x, t) & =-\hat{\mathbf{C}} \frac{\partial}{\partial t} \hat{\boldsymbol{v}}(x, t) .
\end{aligned}
$$

The time-domain solution of (3.18) is [7]

$$
\begin{gathered}
\hat{\boldsymbol{v}}_{0}(t)-\hat{\mathbf{Z}} \hat{\boldsymbol{i}}_{0}(t)=\boldsymbol{w}_{1}(t) \\
\hat{\boldsymbol{v}}_{d}(t)-\hat{\mathbf{Z}} \hat{\boldsymbol{i}}_{d}(t)=\boldsymbol{w}_{2}(t) \\
\boldsymbol{w}_{1}(t)=2 \hat{\boldsymbol{v}}_{d}(t-\boldsymbol{\tau})-\boldsymbol{w}_{2}(t-\boldsymbol{\tau}) \\
\boldsymbol{w}_{2}(t)=2 \hat{\boldsymbol{v}}_{0}(t-\boldsymbol{\tau})-\boldsymbol{w}_{1}(t-\boldsymbol{\tau}),
\end{gathered}
$$

where the delay is $\tau_{i}=d \sqrt{\hat{l}_{i} \hat{c}_{i}}$ and the vectors that are functions of $(t-\boldsymbol{\tau})$ are defined as

$$
\boldsymbol{f}(t-\boldsymbol{\tau})=\left[f_{1}\left(t-\tau_{1}\right) \ldots f_{N}\left(t-\tau_{N}\right)\right]^{T} .
$$

The matrix $\hat{\mathbf{Z}}=\operatorname{diag}\left(\sqrt{\frac{\hat{l}_{1}}{\hat{c}_{1}}, \frac{\hat{l}_{2}}{\hat{c}_{2}}, \ldots, \frac{\hat{l}_{N}}{\hat{c}_{N}}}\right)$ contains the characteristic impedances for each of the $N$ modes. Since (3.21) and (3.22) describe the system in the time-domain, a 
modified nodal analysis (MNA) formulation can be implemented in standard SPICE simulators. Fig. 3.3 shows the equivalent circuit for this system, which is modeled using the modal transformation matrices, $\mathbf{E}_{V}$ and $\mathbf{E}_{I}$, and the sources $\boldsymbol{w}_{1}(t)$ and $\boldsymbol{w}_{2}(t)$. Each of the $i$ subcircuits corresponds to the $i^{\text {th }}$ element in the associated vector.

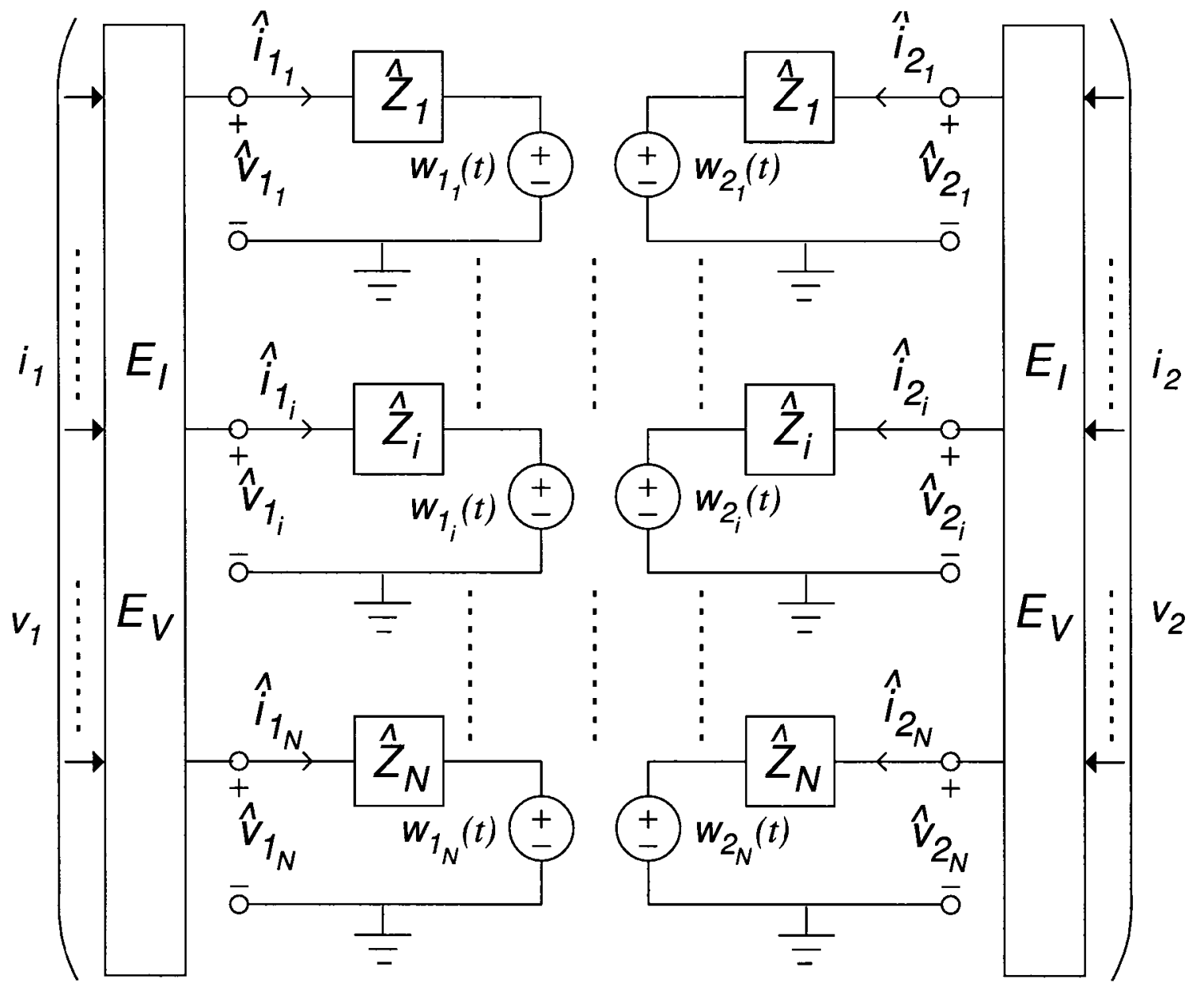

Fig. 3.3: Equivalent circuit representation for the realization of lossless coupled lines. 
TABLE 3.1: A summary of the CPU performance of the macromodels

\begin{tabular}{|l|c|c|c|}
\hline & $\begin{array}{c}\text { Proposed } \\
\text { (seconds) }\end{array}$ & $\begin{array}{c}\text { Vector Fit } \\
\text { (seconds) }\end{array}$ & $\begin{array}{c}\text { Speed-up of } \\
\text { Proposed }\end{array}$ \\
\hline \hline Example 1: Two-port transmission line & 5.65 & 26.05 & $\mathbf{5}$ \\
\hline Example 2: Four-port transmission line & 2.33 & 37.19 & $\mathbf{1 6}$ \\
\hline
\end{tabular}

\subsection{Numerical Results}

This section presents several numerical examples that validate the accuracy and efficiency of the proposed algorithm. The proposed algorithm is compared to the conventional FD-VF algorithm for two frequency-dependent transmission lines. A summary of the results is shown in Table 3.1. Transient simulations were performed using HSPICE on an AMD Athlon $1.83 \mathrm{GHz}$ CPU.

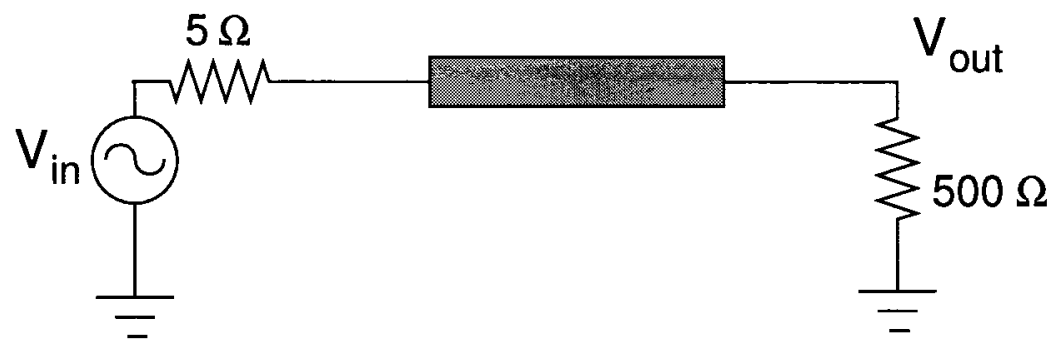

Fig. 3.4: Circuit for example 1.

\subsubsection{Example 1: Two-Port Transmission Line}

In order to validate the proposed macromodel, it is tested on the tabulated frequencydomain data of a two-port transmission line shown in Fig. 3.4. The FD-VF algorithm required 175 poles to fit the given admittance parameters. In comparison, the proposed macromodel required 110 sections to fit the given data. Sample comparisons of the frequency-domain fittings are shown in Fig. 3.5. It is clear that the proposed 


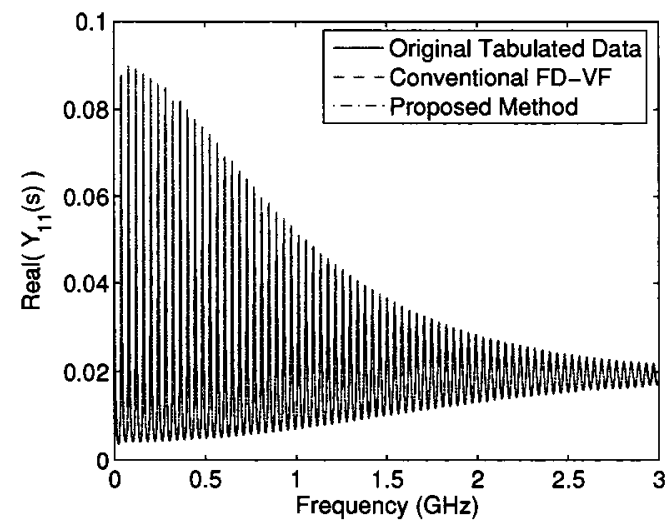

(a) Real part of $Y_{11}(s)$.

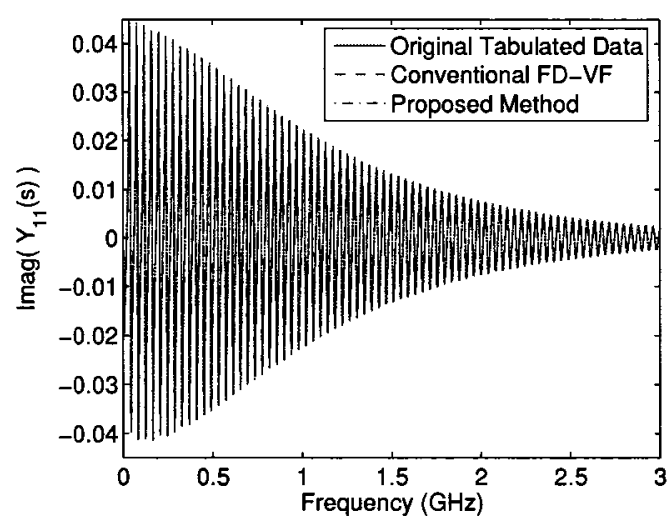

(c) Imaginary part of $Y_{11}(s)$.

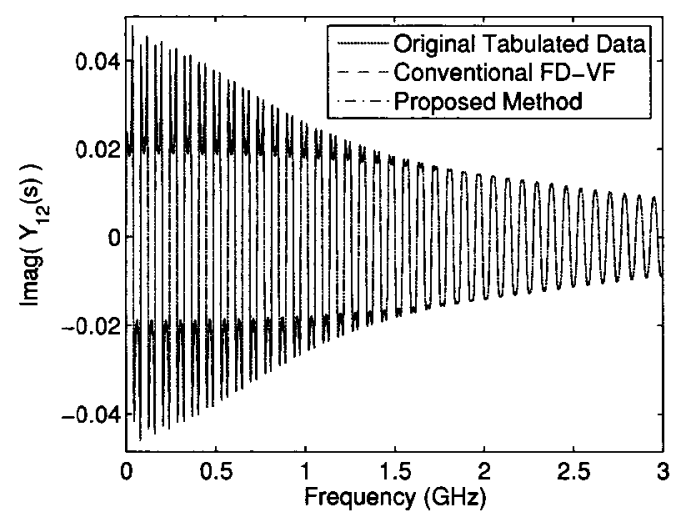

(e) Imaginary part of $Y_{12}(s)$.

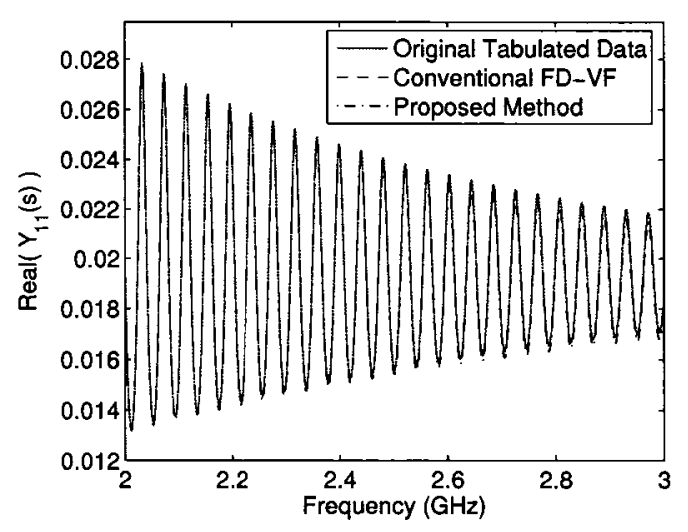

(b) An expanded view of Fig. 3.5(a).

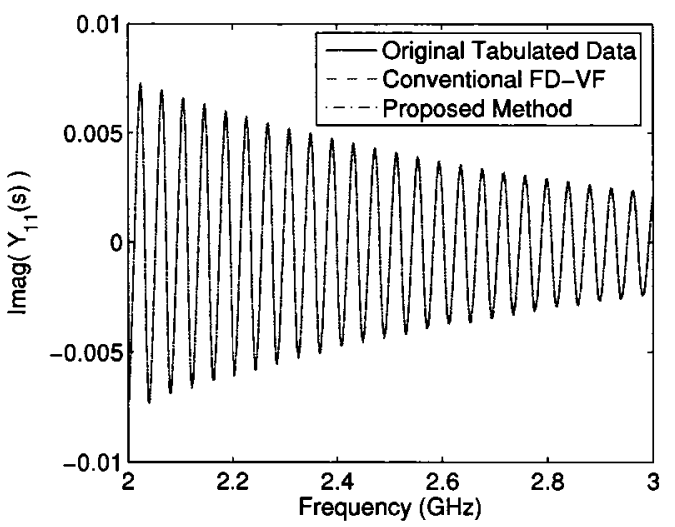

(d) An expanded view of Fig. 3.5(c).

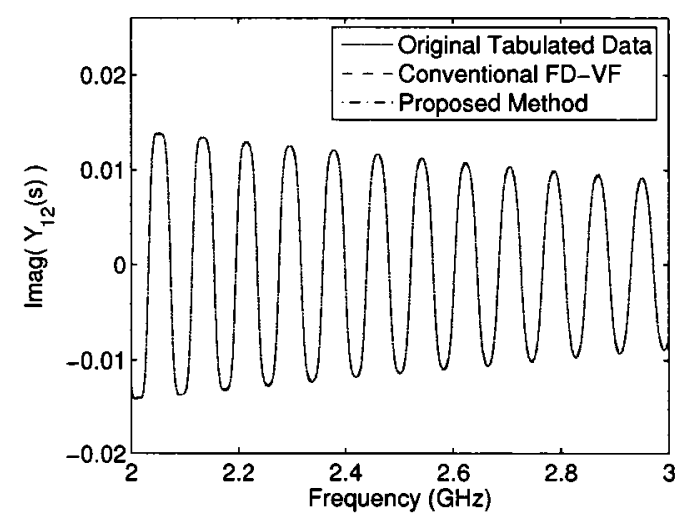

(f) An expanded view of Fig. 3.5(e).

Fig. 3.5: Frequency-domain comparisons for example 1. 


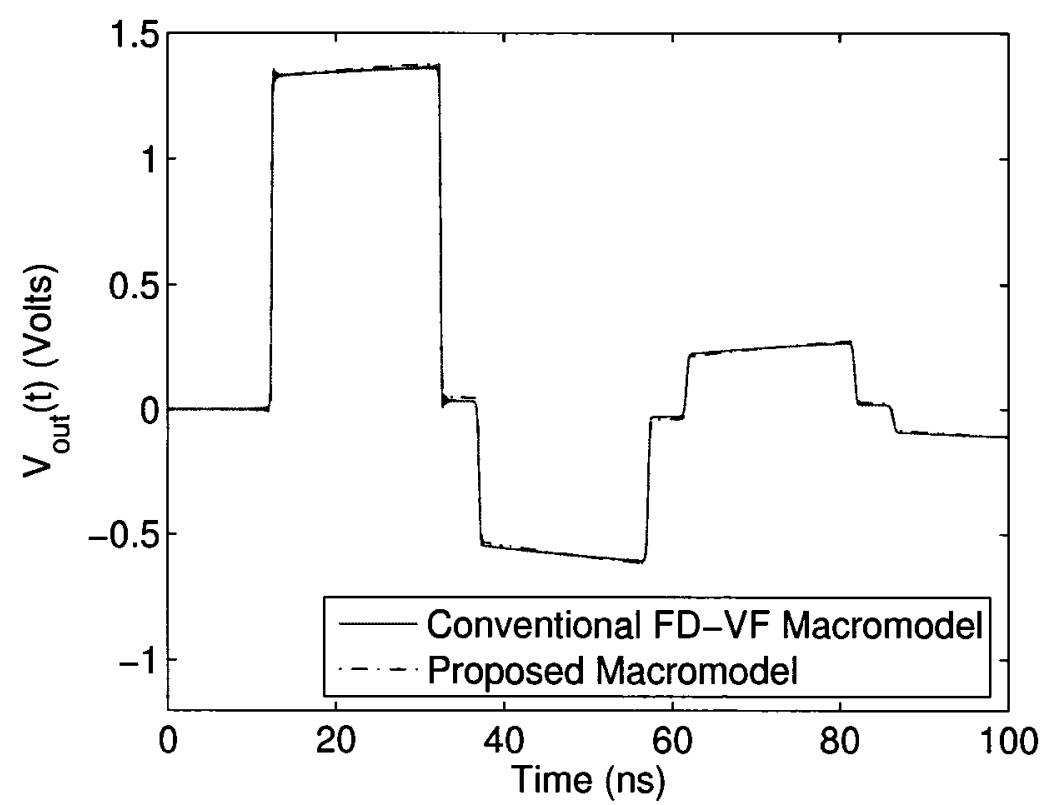

(a) Complete response.

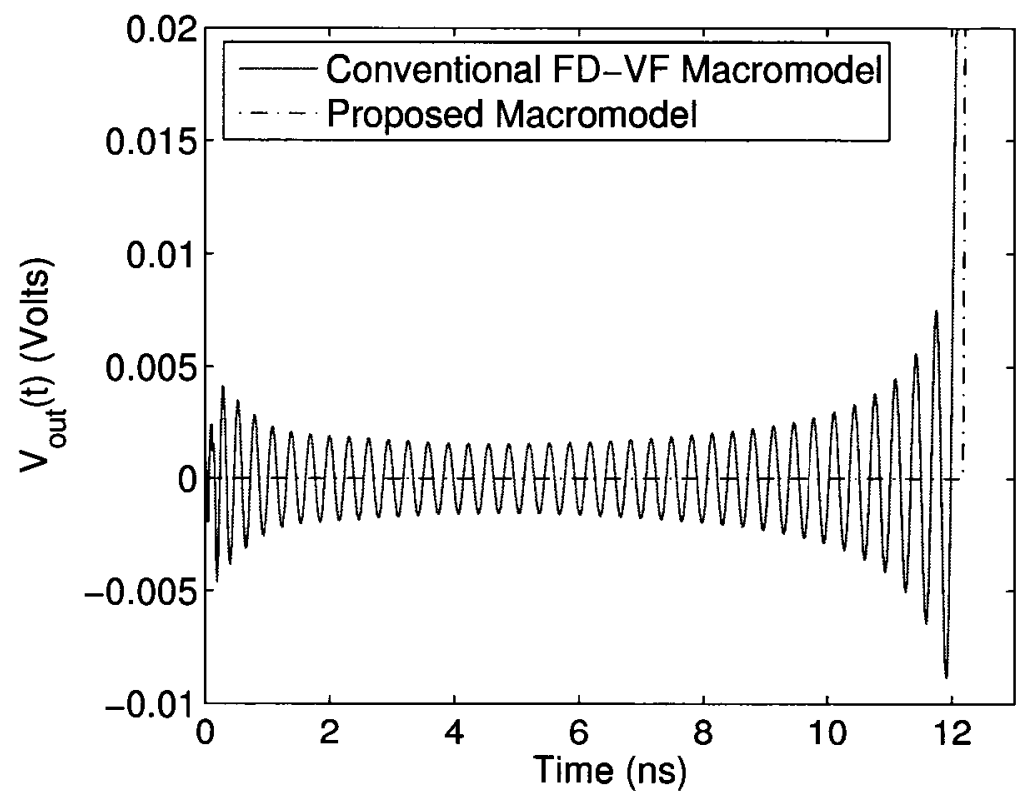

(b) An expanded view of Fig. 3.6(a).

Fig. 3.6: Comparison of transient response for example 1. 
fitting is in good agreement with the original data.

Next, macromodels from both the conventional FD-VF algorithm and the proposed algorithm were simulated in HSPICE. Each network was excited by a pulse with $0.1 n s$ rise/fall times and a pulse width of 20ns. The conventional FD-VF model required 26.05 seconds, compared to 5.65 seconds for the proposed macromodel. A comparison of the transient responses is shown in Fig. 3.6. An expanded view of the response in Fig. 3.6(b) shows the proposed macromodel overcomes the ringing in the response associated with the conventional FD-VF macromodel.

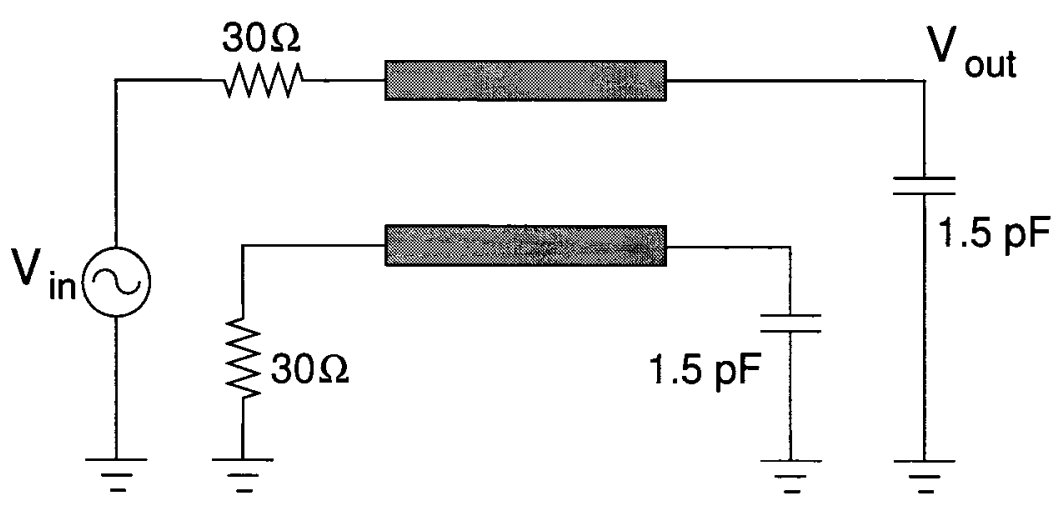

Fig. 3.7: Circuit for example 2.

\subsubsection{Example 2: Four-Port Transmission Line}

In this example, we consider the four-port transmission line shown in Fig. 3.7, characterized by tabulated frequency-domain admittance parameters. The conventional FD-VF approximation required a common pole set of 146 poles to fit the admittance response matrix. In comparison, the proposed algorithm required 38 sections in the MLF approximation to fit the admittance response matrix. As seen in Fig. 3.8, the conventional FD-VF and the proposed fitting are in good agreement with the original 


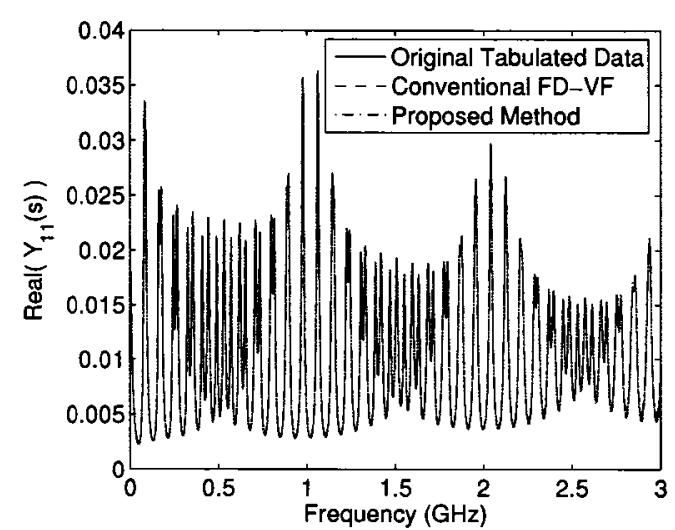

(a) Real part of $Y_{11}(s)$.

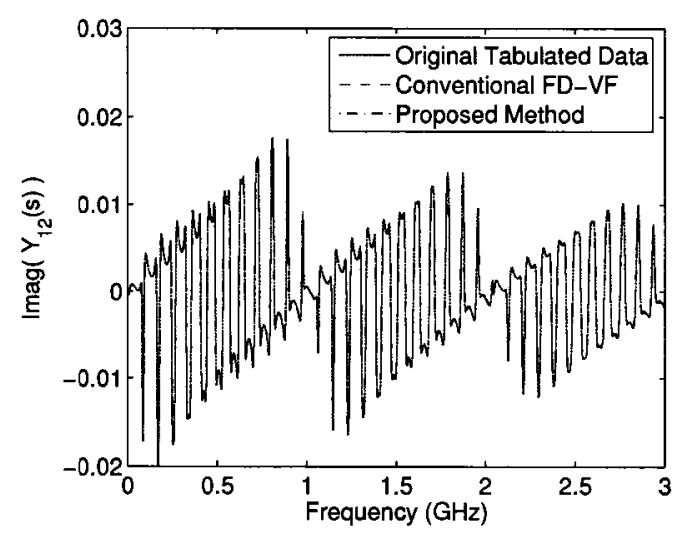

(c) Imaginary part of $Y_{12}(s)$.

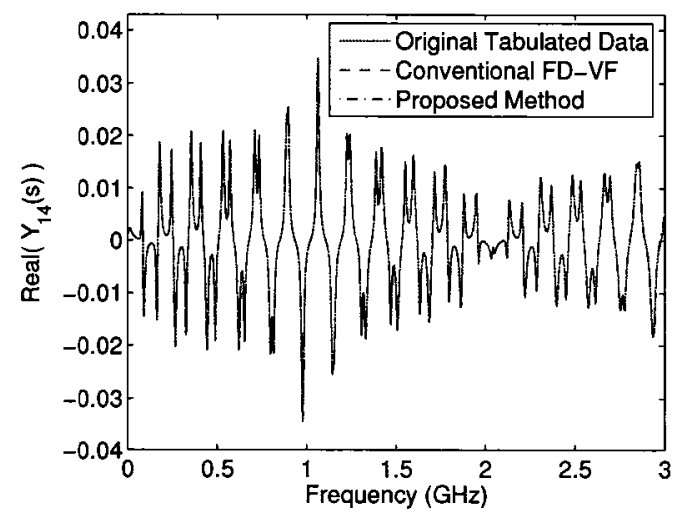

(e) Real part of $Y_{14}(s)$.

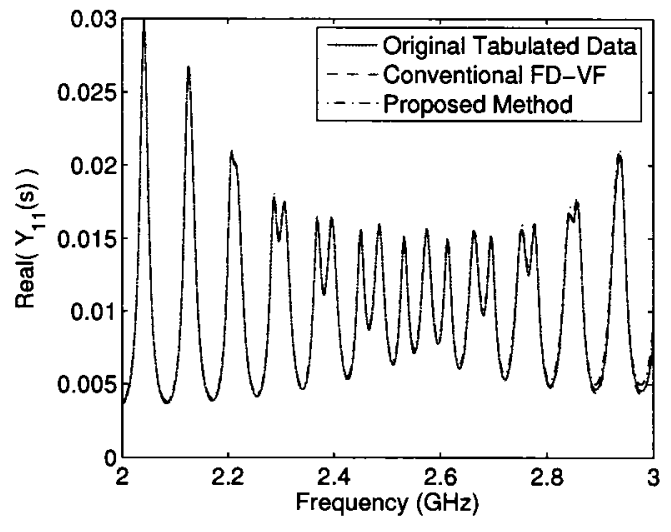

(b) An expanded view of Fig. 3.8(a).

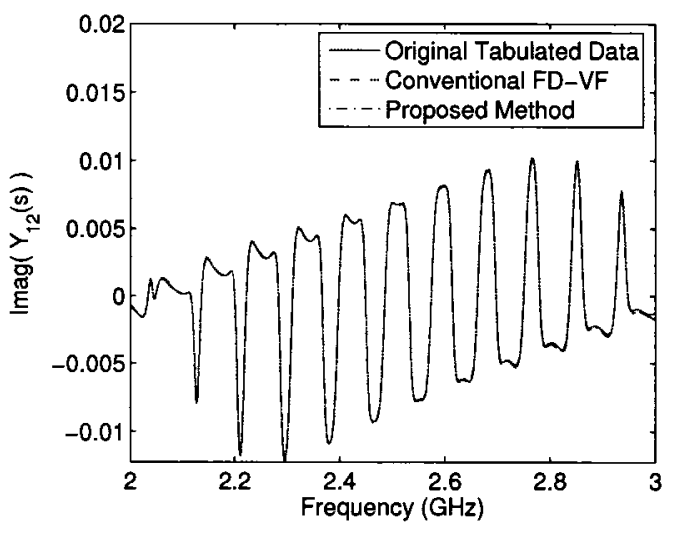

(d) An expanded view of Fig. 3.8(c).

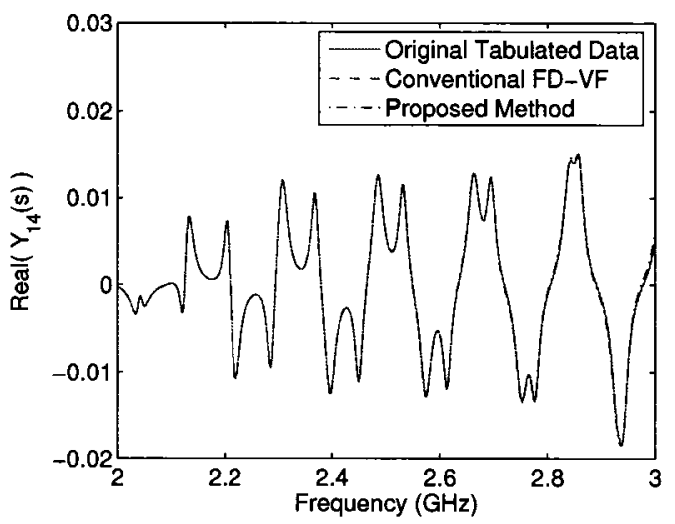

(f) An expanded view of Fig. 3.8(e).

Fig. 3.8: Frequency-domain comparisons for example 2. 


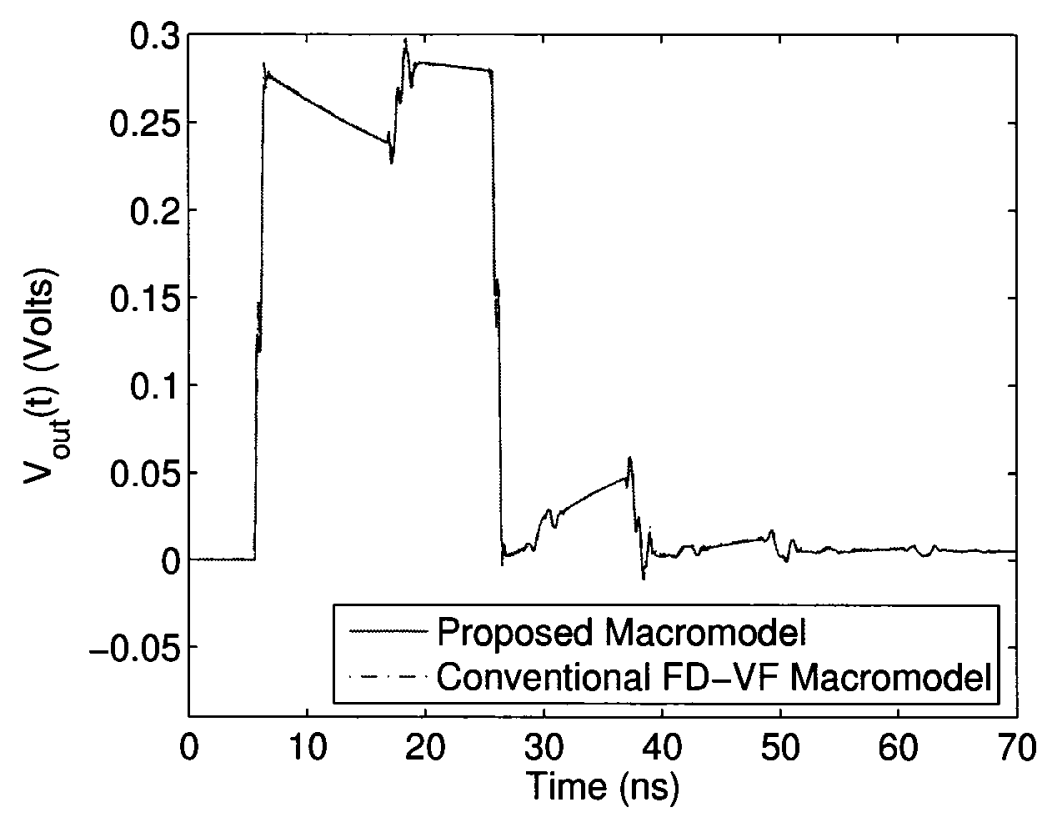

Fig. 3.9: Comparison of transient response for example 2.

data.

Next, macromodels from both the conventional FD-VF and the proposed algorithm were simulated in HSPICE. Each macromodel was excited by a pulse with $0.1 n s$ rise/fall times and $20 \mathrm{~ns}$ pulse width. The conventional FD-VF model required 37.19 seconds to simulate, compared to 2.33 seconds for the proposed macromodel. A sample comparisons of the transient response is shown in Fig. 3.9. As seen, the transient response from both FD-VF macromodel and the proposed macromodel are in good agreement. 


\subsection{Summary}

In this chapter, a new method has been introduced for the construction of a compact macromodel from tabulated frequency-domain data characterizing a single set of transmission lines. This method extends the DEPACT algorithm to cases where the $\mathrm{Y}, \mathrm{Z}, \mathrm{S}$, or $\mathrm{H}$ parameters are given instead of the p.u.l parameters. The algorithm solves a least squares system to obtain a unique logarithm of the hybrid parameters. Next, the DEPACT methodology is used to obtain a SPICE macromodel. The resulting macromodel is significantly faster than the popular vector fitting algorithm [30], which requires a large number of poles for long delay lines. 


\section{Chapter 4}

\section{Macromodeling via Delayed}

\section{Rational Functions Using Prony's}

\section{Algorithm}

\subsection{Introduction}

In the previous chapter, a macromodel was developed for a single set of transmission lines characterized by tabulated data in the form of $\mathrm{H}, \mathrm{S}, \mathrm{Y}$, or $\mathrm{Z}$ parameters. However, in many instances tabulated data is adopted to model more complex interconnect networks. In such cases, order reduction methods for rational function based approximation of the data have been suggested [23]-[27]. These methods determine only a single delay from functions representing a port-to-port response. However, if multiple reflections and delays are present in the system, then these methods do not significantly reduce the size of the resulting macromodels. Recently, time-frequency 
analysis using Gabor transform based delay estimation was proposed [29] for long delay lines characterized by tabulated frequency-domain data.

This chapter develops a novel algorithm for low-order (compact) macromodeling of electrically long high-speed circuits characterized by tabulated impulse port response data. The algorithm first partitions the time-domain impulse response data into several intervals. It then approximates each interval with a sum of delayed exponentials in the time-domain. The new method leads to highly compact (low-order) macromodels.

This chapter is organized as follows. Section 4.2 develops the proposed algorithm. Section 4.3 presents wavelet decomposition based time-frequency analysis for delay determination. Next, section 4.4 describes continuity enforcement in the delayed rational formulation. Sections 4.5 and 4.6 present some numerical results and conclusions, respectively.

\subsection{Development of the Proposed Algorithm}

In this section, a novel algorithm is presented to overcome the difficulty associated with the macromodeling of high-speed circuits with long delays. In the proposed algorithm, the time-domain impulse data is divided into several partitions and each partition is approximated with a sum of delayed exponential functions using methods such as Prony's [62] or other appropriate time-domain approximation methods. This leads to low-order delayed rational functions, which can be converted into a system of DDEs for SPICE simulation. To understand the proposed algorithm, consider the time-domain waveform of Fig. 4.1. The waveform is divided into $Q$ intervals such 
that $t_{0}<t_{1}<\ldots<t_{n} \ldots<t_{Q}$ as shown. Each partition, $\phi_{n}(t)$, is approximated

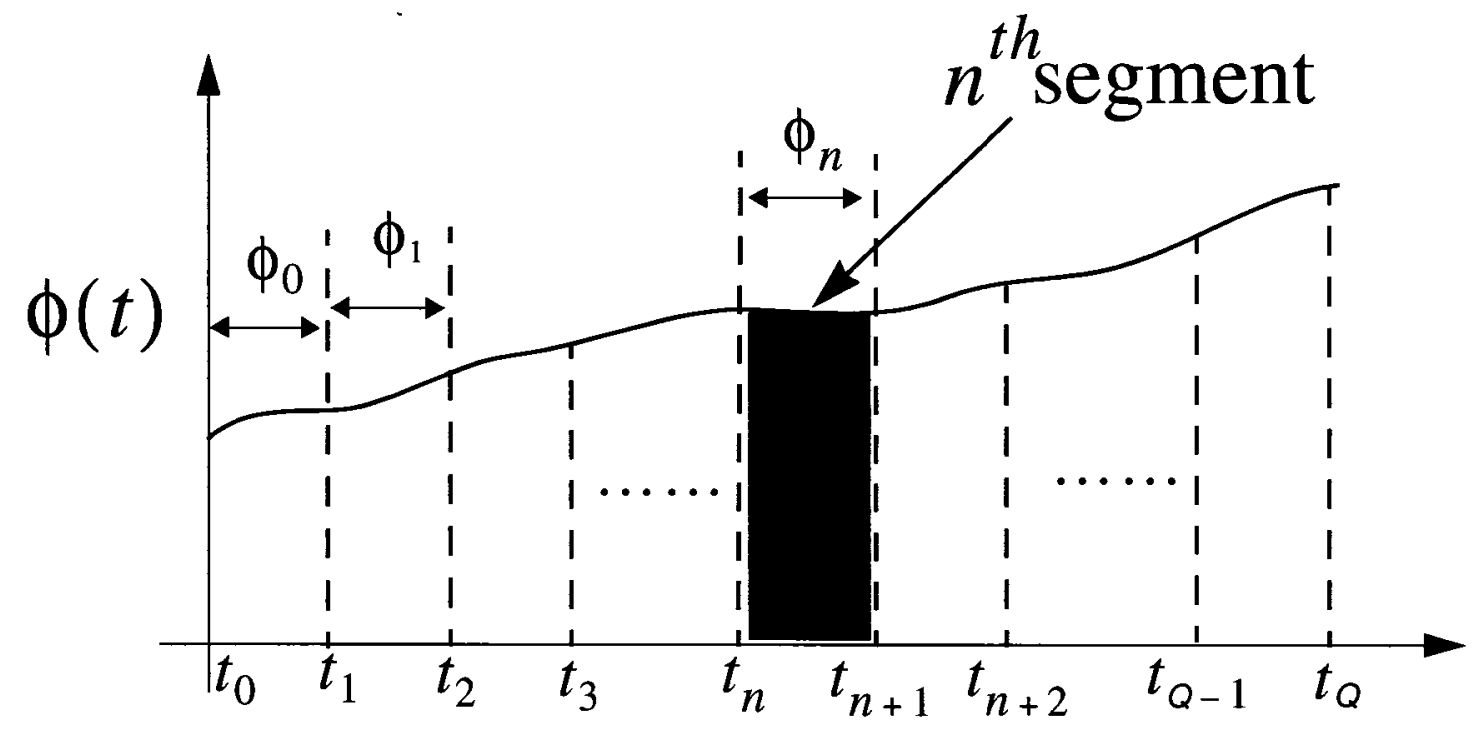

Fig. 4.1: Segmentation of the time-domain waveform into $Q$ intervals.

using a sum of $M_{n}$ exponentials of the form

$$
\phi_{n}(t)=\sum_{i=1}^{M_{n}} R_{n, i} e^{p_{n, i}\left(t-t_{n}\right)}\left(u\left(t-t_{n}\right)-u\left(t-t_{n+1}\right)\right) .
$$

Here, $\left\{p_{n, i}\right\}$ and $\left\{R_{n, i}\right\}$ are the poles and residues, respectively, for the $n^{\text {th }}$ partition. Using this strategy, the complete waveform can be expressed as a sum of the waveforms in each interval as

$$
\phi(t)=\sum_{n=0}^{Q-1} \sum_{i=1}^{M_{n}} R_{n, i} e^{p_{n, i}\left(t-t_{n}\right)}\left(u\left(t-t_{n}\right)-u\left(t-t_{n+1}\right)\right)
$$


After certain mathematical manipulations, the frequency-domain expression for this waveform can then be found by taking the Laplace transform of (4.2) as

$$
\begin{aligned}
\Phi(s) & =\mathfrak{L}\{\phi(t)\} \\
& =\mathfrak{L}\left\{\sum_{n=0}^{Q-1} \sum_{i=1}^{M_{n}} R_{n, i} e^{p_{n, i}\left(t-t_{n}\right)}\left(u\left(t-t_{n}\right)-u\left(t-t_{n+1}\right)\right)\right\} \\
& =\sum_{n=0}^{Q-1} \sum_{i=1}^{M_{n}}\left(\frac{R_{n, i} e^{-s t_{n}}}{s-p_{n, i}}-\frac{R_{n, i} e^{p_{n, i}\left(t_{n+1}-t_{n}\right)} e^{-s t_{n+1}}}{s-p_{n, i}}\right) .
\end{aligned}
$$

After approximating each entry of the transfer function matrix in the form of delayed rational functions (4.3), a macromodel in the form of DDEs can be derived for use in conventional simulators. Two important challenges that need to be addressed during partitioning and subsequent approximations are the following.

1. Determination of partition points.

2. Enforcing continuity in the approximation of consecutive partitions.

These challenges are addressed in the following sections.

\subsection{Determination of Partition Points}

In the proposed algorithm, the interval (or split) points are determined by performing a time-frequency analysis of the time-domain impulse response data using wavelet decomposition. For the benefit of the reader, a review of wavelet theory, as relevant to this thesis, is given in appendix A. To begin, a boundary adapted forward wavelet transform is applied to the impulse response data, $\{h(t)\}$ [63]. For wavelet and scaling basis functions with $M$ filter coefficients on the interval $0 \leq k<M / 2$, the transform 
becomes

$$
\begin{aligned}
& a_{j}\left(t_{k}\right)=\sum_{l=0}^{M / 2-1} H_{k, l}^{l e f t} a_{j-1}\left(t_{l}\right)+\sum_{m=M / 2}^{M / 2-2 k} h_{k, m}^{l e f t} a_{j-1}\left(t_{m}\right), \\
& d_{j}\left(t_{k}\right)=\sum_{l=0}^{M / 2-1} G_{k, l}^{l e f t} a_{j-1}\left(t_{l}\right)+\sum_{m=M / 2}^{M / 2-2 k} g_{k, m}^{l e f t} a_{j-1}\left(t_{m}\right),
\end{aligned}
$$

where $H_{k, l}^{l e f t}, h_{k, m}^{l e f t}, G_{k, l}^{l e f t}$, and $g_{k, m}^{l e f t}$ are the filter coefficients for the left boundary wavelet and scaling functions, respectively. $a_{j}$ and $d_{j}$ are the approximation and detail coefficients, respectively, for the $j^{\text {th }}$ resolution level with $a_{1}\left(t_{k}\right)=h\left(t_{k}\right)$. If $M / 2<k \leq n-M / 2$, then

$$
\begin{aligned}
a_{j}\left(t_{k}\right) & =\sum_{l=0}^{M-1} h_{l} a_{j-1}\left(t_{l}\right), \\
d_{j}\left(t_{k}\right) & =\sum_{l=0}^{M-1} g_{l} a_{j-1}\left(t_{l}\right),
\end{aligned}
$$

where $h_{l}$ and $g_{l}$ are the filter coefficients and $n$ is the total number of data points. Finally, if $-M / 2 \leq k<0$

$$
\begin{aligned}
& a_{j}\left(t_{n+k}\right)=\sum_{l=-M / 2}^{-1} H_{k, l}^{r i g h t} a_{j-1}\left(t_{n / 2+l}\right)+\sum_{m=-M / 2+2 k+1}^{-M / 2-1} h_{k, m}^{r i g h t} a_{j-1}\left(t_{n / 2+m}\right), \\
& d_{j}\left(t_{n+k}\right)=\sum_{l=-M / 2}^{-1} G_{k, l}^{r i g h t} a_{j-1}\left(t_{n / 2+l}\right)+\sum_{m=-M / 2+2 k+1}^{-M / 2-1} g_{k, m}^{r i g h t} a_{j-1}\left(t_{n / 2+m}\right),
\end{aligned}
$$

where $H_{k, l}^{r i g h t}, h_{k, m}^{r i g h t}, G_{k, l}^{r i g h t}$, and $g_{k, m}^{r i g h t}$ are filter coefficients for the right boundary wavelet and scaling functions, respectively.

The forward wavelet transform acts as a filter, with the low frequency content stored in the approximation coefficients, and the high frequency content in the detail 
coefficients. The detail coefficients of the largest magnitude correspond to the highest frequency content, and these points provide the information on the partition points. Clusters of points will arise in the regions pertaining to impulse functions (since this is impulse response data, and impulses pertain to very high frequency content). The first and last point of each cluster is chosen as the final partition points to capture all the high frequency content in contained intervals. The remaining partitions without high frequency content (between the clusters) can be fit with low-order approximations. While the clustered regions require slightly higher order approximations, the approximations are still of much lower order than if the entire impulse response was fit directly. A detailed discussion of this process is given in section 4.5.

The algorithm developed thus far obtains final partition regions that require loworder approximations relative to fitting the entire data set directly. However, it does not ensure that the resulting approximation is continuous at the partition points.

\subsection{Continuity Enforcement}

It is to be noted that the separate approximation of various intervals of a continuous waveform can lead to discontinuities at the time points of overlap between adjoining partitions (at time points $t_{1}, t_{2}, \ldots, t_{Q-1}$ in Fig. 4.1). This can happen if the waveform partitions $\phi_{0}(t), \phi_{1}(t), \ldots, \phi_{Q-1}(t)$ are each approximated independently. In order to overcome this problem, a modification is made to the least squares step for the calculation of residues, to enforce $\phi_{n}\left(t_{n}\right)=\phi_{n-1}\left(t_{n}\right)$. The standard least squares 
problem to find the residues for $\phi_{n}(t)$ is the solution of

$$
\phi_{n}(t)=\sum_{i=1}^{M_{n}} R_{n, i} e^{p_{n, i}\left(t-t_{n}\right)}\left(u\left(t-t_{n}\right)-u\left(t-t_{n+1}\right)\right)
$$

At time, $t_{n}$, enforcing $\phi_{n}\left(t_{n}\right)=\phi_{n-1}\left(t_{n}\right)$ yields

$$
\sum_{i=1}^{M_{n}} R_{n, i}=\phi_{n-1}\left(t_{n}\right)
$$

Fixing one of the residues will ensure that $\phi_{n}\left(t_{n}\right)=\phi_{n-1}\left(t_{n}\right)$ holds. If the fixed residue is chosen arbitrarily as $R_{n, 1}$, then (4.11) can be rearranged to obtain

$$
R_{n, 1}=\phi_{n-1}\left(t_{n}\right)-\sum_{i=2}^{M_{n}} R_{n, i}
$$

Substituting (4.12) in (4.10) and solving for the remaining residues yields

$$
\sum_{i=2}^{M_{n}} R_{n, i}\left(e^{p_{n, i}\left(t-t_{n}\right)}-e^{p_{n, 1}\left(t-t_{n}\right)}\right)=\phi_{n}(t)-\phi_{n-1}\left(t_{n}\right) e^{p_{n, 1}\left(t-t_{n}\right)}
$$

Equation (4.13) is solved as a least squares problem for the residues $R_{n, 2}$ to $R_{n, M_{n}}$, which are then substituted in (4.12) to obtain $R_{n, 1}$.

\subsection{Numerical Results}

This section presents several numerical examples assessing the accuracy and efficiency of the proposed algorithm. Comparisons are made between the proposed algorithm and the FD-VF algorithm for several networks. In addition, the proposed macromodel is realized using techniques discussed in chapter 6 for transient SPICE comparisons 
TABLE 4.1: A summary of the CPU performance of the macromodels

\begin{tabular}{|c|c|c|c|}
\hline & $\begin{array}{c}\text { Vector Fit } \\
\text { (seconds) }\end{array}$ & $\begin{array}{c}\text { Proposed } \\
\text { (seconds) }\end{array}$ & $\begin{array}{c}\text { Speed-up of } \\
\text { Proposed }\end{array}$ \\
\hline \hline Example 1 & 29.83 & 22.67 & $\mathbf{1}$ \\
\hline Example 2 & 33.57 & 11.70 & $\mathbf{3}$ \\
\hline Example 3 & 117.36 & 32.98 & $\mathbf{4}$ \\
\hline
\end{tabular}

with the FD-VF macromodel. Example 1 tests the proposed algorithm on the admittance parameters of a two-port transmission line and compares both results to the HSPICE W-Element. Example 2 tests the algorithm on the scattering parameters of an unknown two-port network. Next, example 3 considers a four-port structure described by scattering parameters. A summary of the results is shown in Table 4.1. Transient simulations were performed using HSPICE on an AMD Athlon $1.83 \mathrm{GHz}$ CPU.

\subsubsection{Example 1}

In order to validate and prove the concept of the proposed algorithm, an interconnect circuit with known p.u.l parameters is selected (shown in Fig. 4.2). To validate the accuracy of the proposed algorithm, the proposed macromodel is simulated using HSPICE. The resulting transient response of the network is compared against the transient response by replacing the macromodel with the W-element (of HSPICE) for the interconnect.

The tabulated time-domain impulse response data for $y_{11}(t)$, as well as its timefrequency analysis is shown in Fig. 4.3. In this example, to determine the partitioning points, the forward wavelet transform was applied using orthogonal Daubechies wavelets with four filter coefficients [63], [64]. Consider the wavelet decomposition (in 


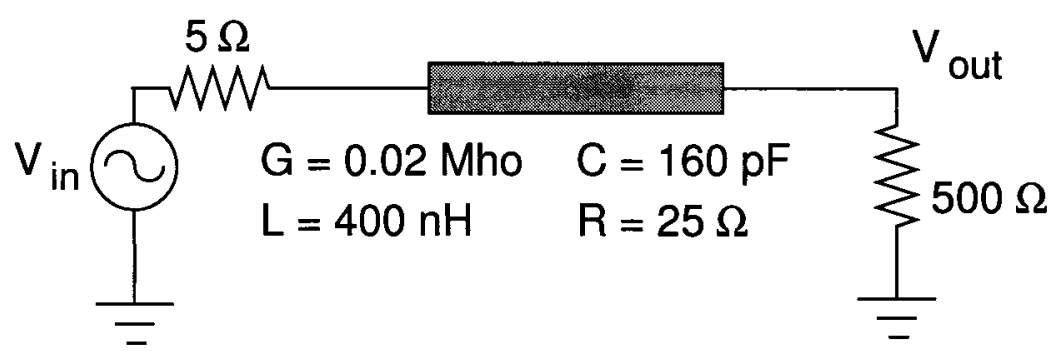

Fig. 4.2: Circuit for example 1.

the form of Heisenberg tiles [63] on the time-frequency plane) shown in Fig. 4.3(a) corresponding to the time-domain impulse response shown in Fig. 4.3(c). To clarify the illustrations, an expanded view of Fig. 4.3(a) and Fig. 4.3(c) between 15.5ns and 16.5ns is given in Fig. 4.3(b) and Fig. 4.3(d), respectively. Darkness of each tile corresponds to the intensity (in $\mathrm{dBv}$ ) of the frequency content at the given location in time. Wavelet coefficients with larger magnitudes and at higher frequency scales are selected as potential splitting points. This information is extracted and the potential split points are shown using ' $x$ ' in Figs. 4.3(c) and 4.3(d), which tend to occur in clusters. To determine the actual split points from the potential split points, clusters of points in regions of about 1 ns - $1.5 \mathrm{~ns}$ (which is significantly shorter than the associated delays of this example) were isolated. The first and last point in each cluster were chosen as the final partition points (to capture all the high frequency content of each impulse in a single partition). The selected partition points for this example are shown by ' $\square$ ' in Fig. 4.3(c) and Fig. 4.3(d).

It required a total of 56 segments and 358 poles to fit the entire network using the proposed algorithm. In comparison, the FD-VF algorithm required a total of 110 common poles to fit the entire network. Sample comparisons of their fittings are shown in Fig. 4.4. 


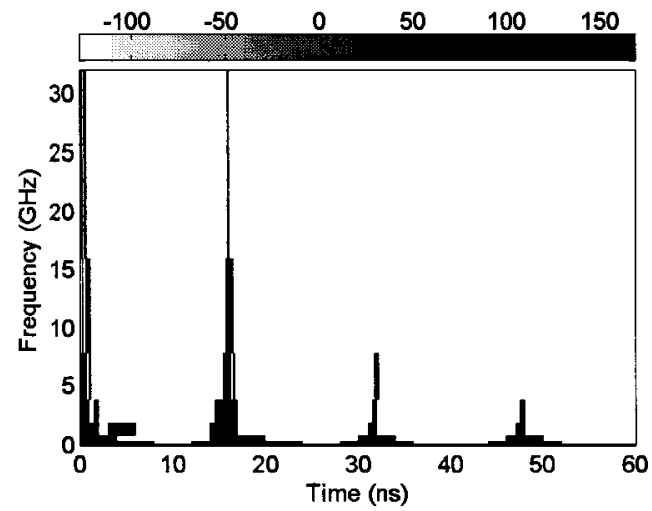

(a) Heisenberg tiles for wavelet decomposition.

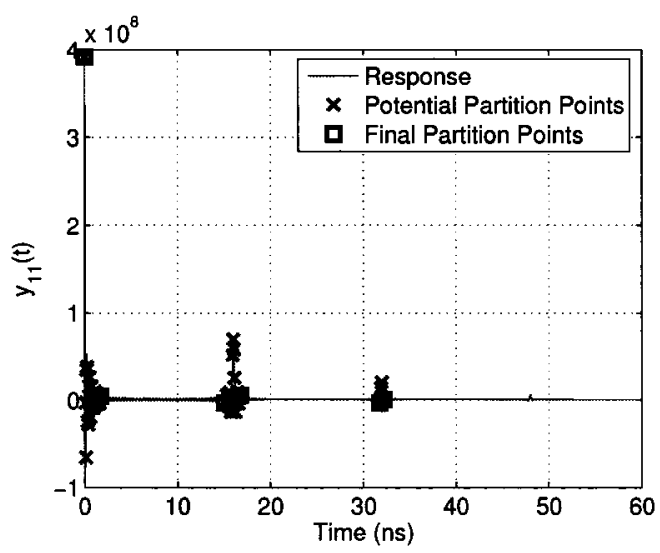

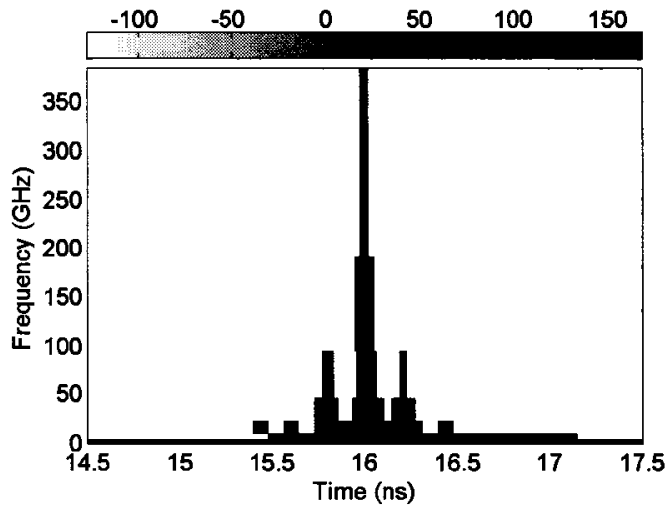

(b) An expanded view of Fig. 4.3(a) between $14.5 n s$ and $17.5 n s$.

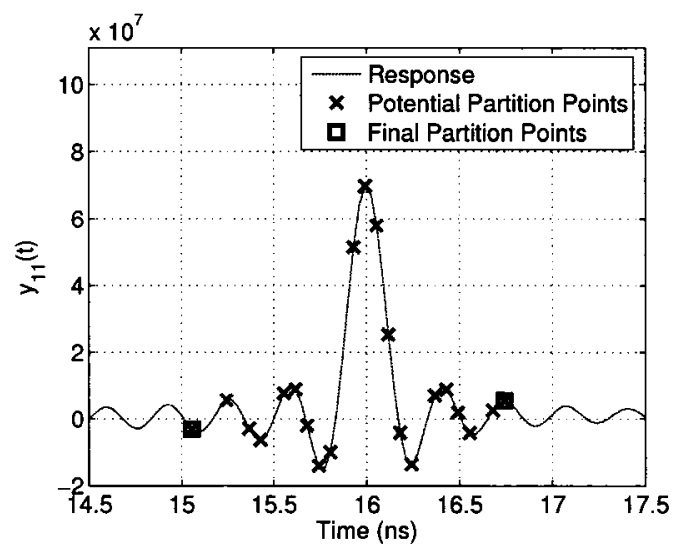

(c) Display of potential partition points and final (d) An expanded view of Fig. 4.3(c) between partition points. $14.5 n s$ and $17.5 n s$.

Fig. 4.3: Time-frequency analysis of the response data. 


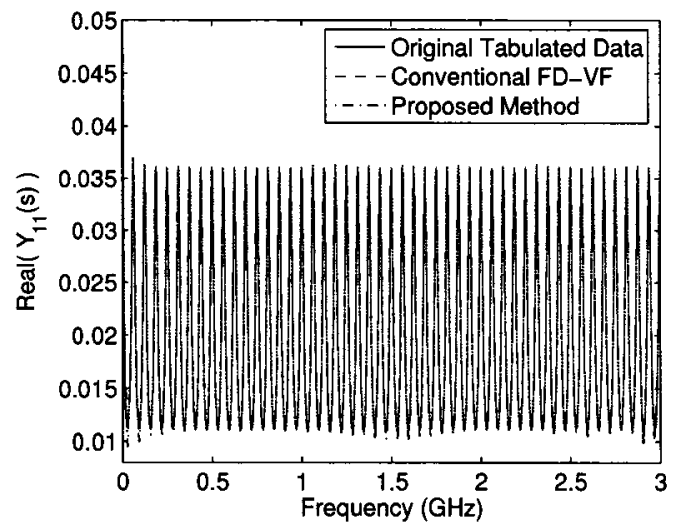

(a) Real part of $Y_{11}(s)$.

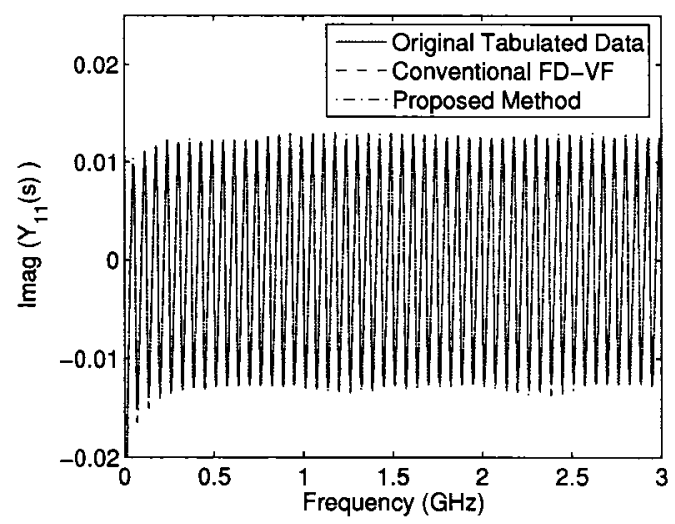

(c) Imaginary part of $Y_{11}(s)$.

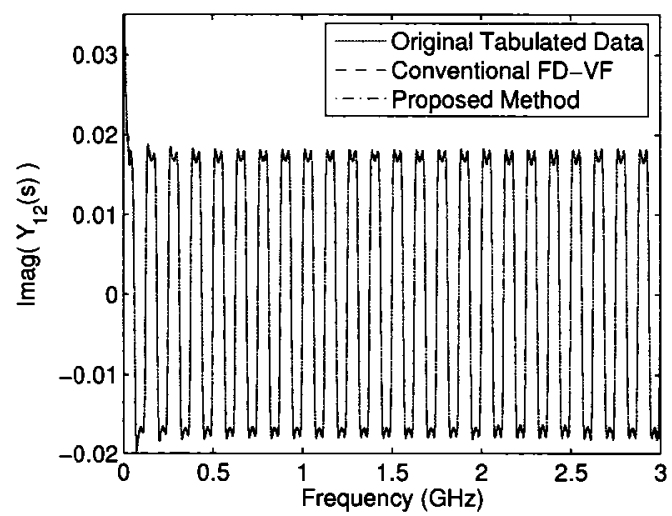

(e) Imaginary part of $Y_{12}(s)$.

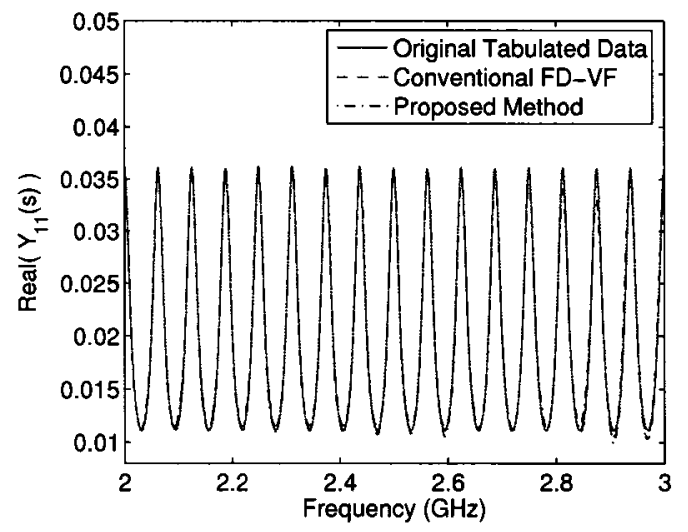

(b) An expanded view of Fig. 4.4(a).

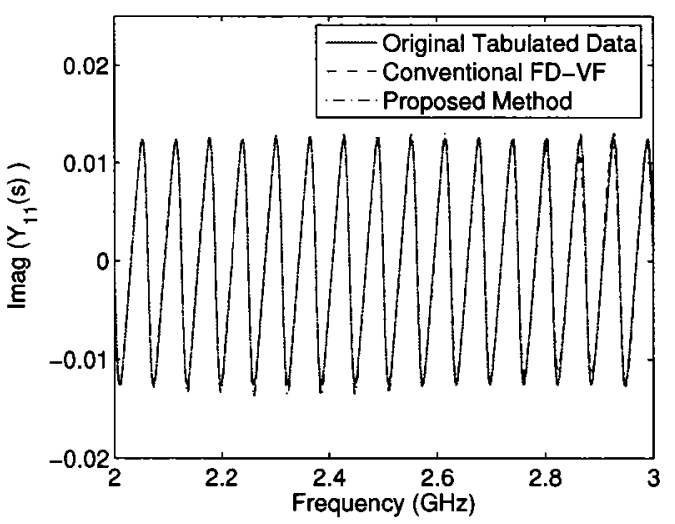

(d) An expanded view of Fig. 4.4(c).

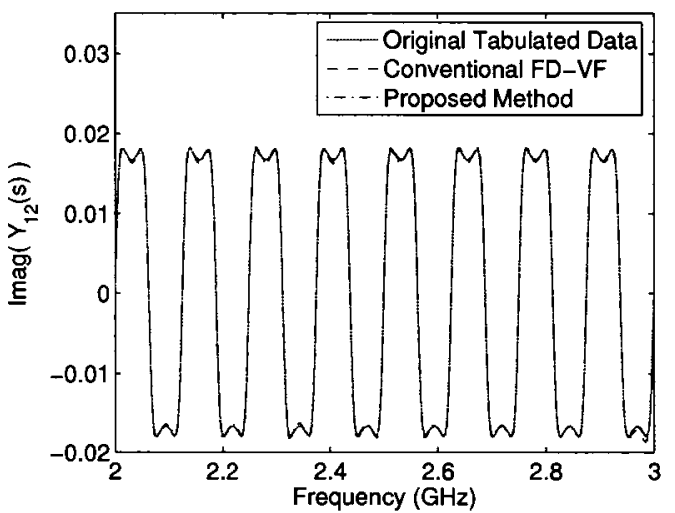

(f) An expanded view of Fig. 4.4(e).

Fig. 4.4: Frequency-domain comparisons for example 1. 
Next, the macromodels from both FD-VF and the proposed algorithm are simulated in HSPICE. Each macromodel was excited by a $20 \mathrm{~ns}$ wide pulse with $0.1 \mathrm{~ns}$ rise/fall times. It required 29.83 seconds to simulate the FD-VF macromodel, compared to 22.67 seconds for the proposed macromodel. A comparison of the transient responses is shown in Fig. 4.5. As seen, the results from both the FD-VF macromodel and the proposed macromodel are in good agreement with the benchmark response.

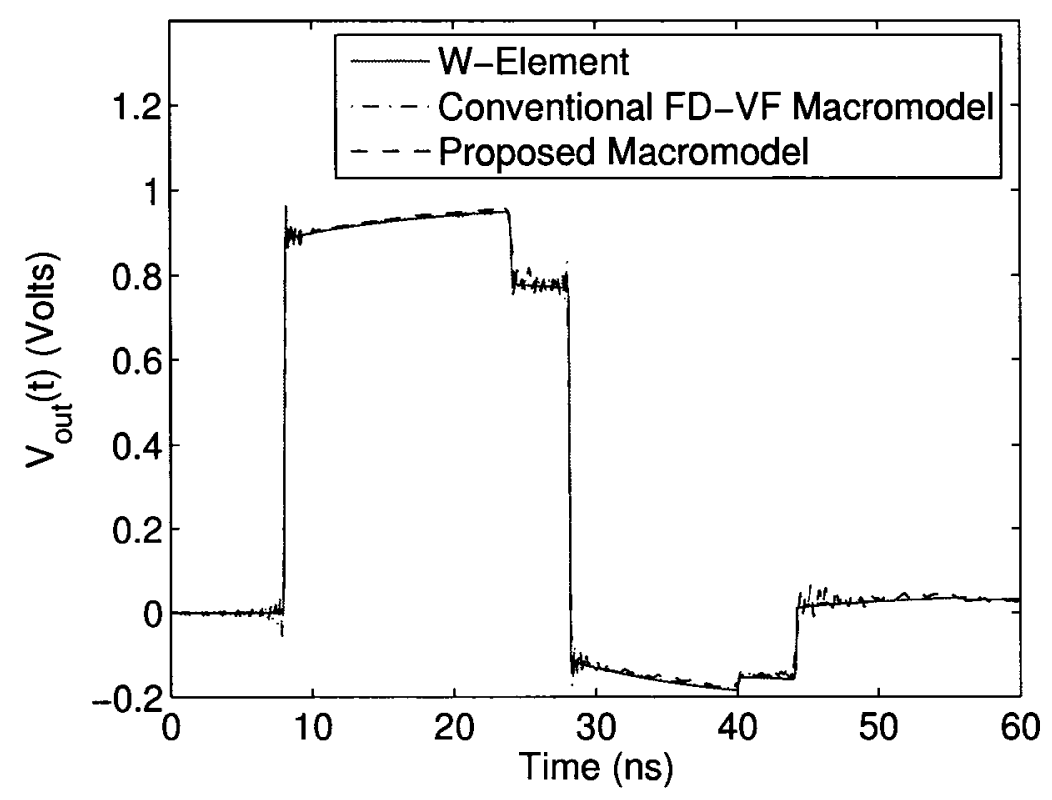

Fig. 4.5: Transient comparison for example 1.

\subsubsection{Example 2}

In this example, the proposed algorithm is applied to the time-domain impulse response data describing the scattering parameters for a two-port network (see Fig. 4.6). The time-domain impulse response, as well as the time-frequency analysis for $s_{12}(t)$ are shown in Fig. 4.7. The proposed algorithm required a total of 47 seg- 
ments and 225 poles to fit the response data for the entire network. In comparison, the FD-VF algorithm required a total of 480 poles for the entire network. Sample comparisons for the frequency-domain scattering parameter fittings are shown in Fig. 4.8 .

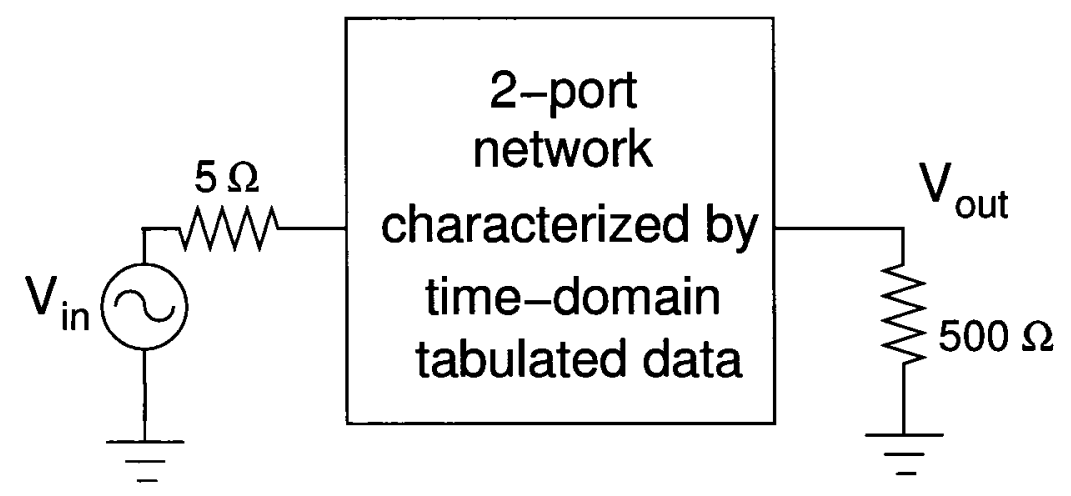

Fig. 4.6: Circuit for example 2.

Next, macromodels from both the FD-VF algorithm, as well as the proposed algorithm were simulated in HSPICE. Each macromodel was excited with a pulse of $0.1 n s$ rise/fall time and width of $20 \mathrm{~ns}$. The FD-VF macromodel required 33.57 seconds to simulate. In comparison, the proposed macromodel required 11.70 seconds to obtain a transient solution. A comparison of the transient responses is shown in Fig. 4.9. As seen, both results are in agreement, however, the proposed response contains less ringing in the response.

\subsubsection{Example 3}

In this example, a four-port network is used to validate the accuracy of the proposed algorithm (shown in Fig. 4.10). A sample time-frequency analysis of the time-domain impulse response scattering data for $s_{13}(t)$ is shown in Fig. 4.11. The proposed 

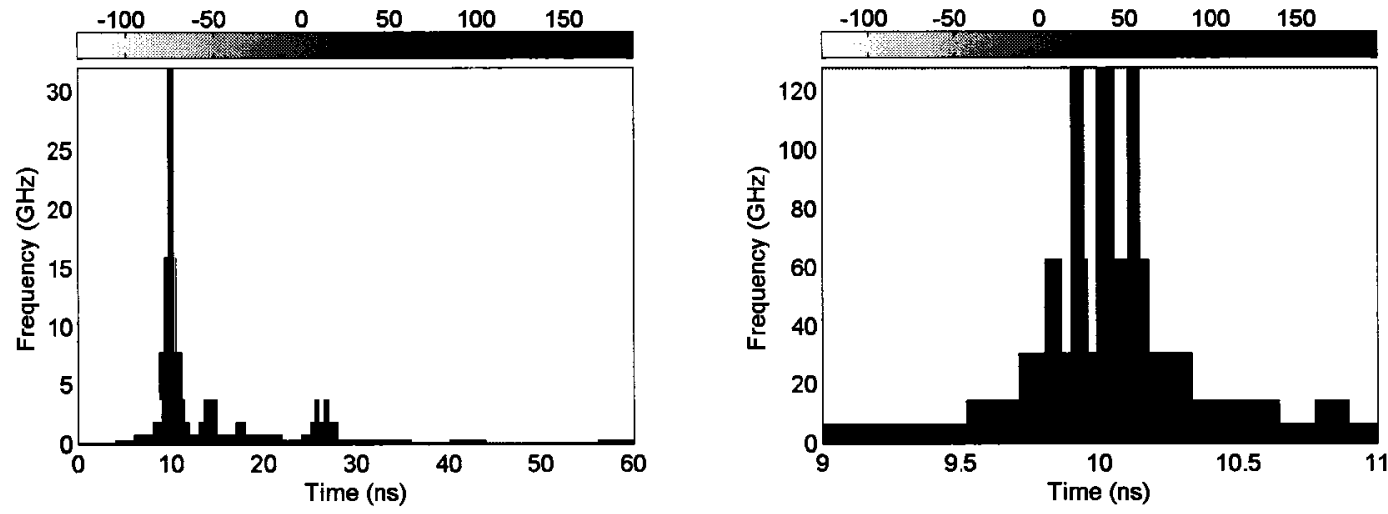

(a) Heisenberg tiles for wavelet decomposition.

(b) An expanded view of Fig. 4.7(a) between $9 \mathrm{~ns}$ and $11 n s$.
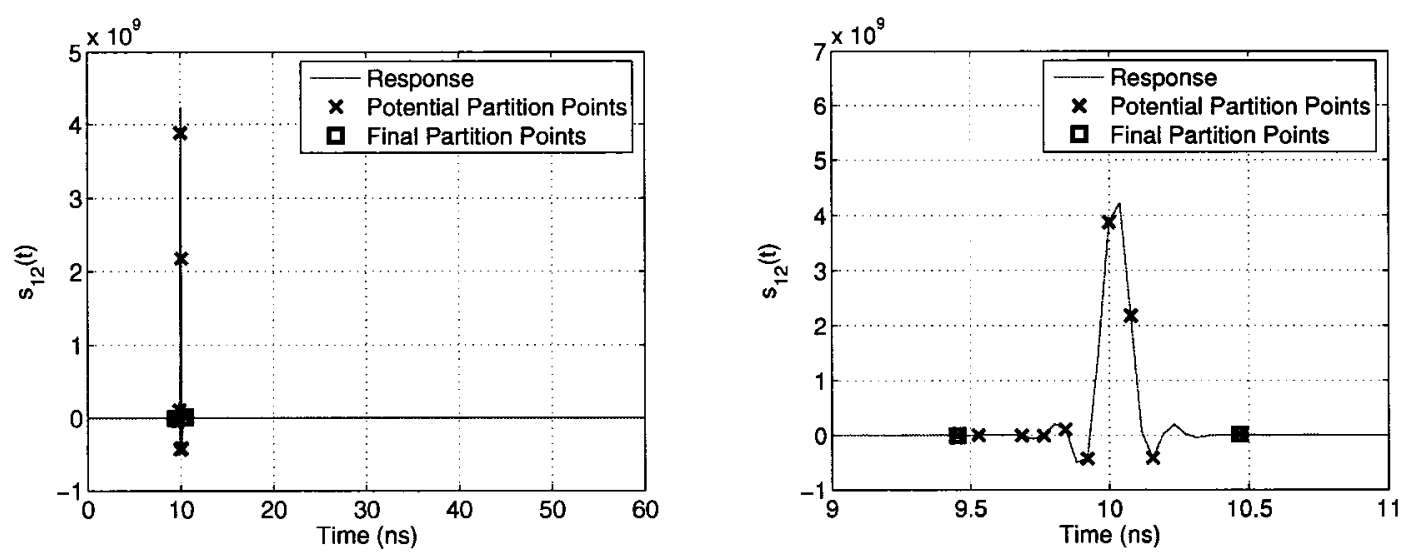

(c) Display of potential partition points and final (d) An expanded view of Fig. 4.7(c) between $9 \mathrm{~ns}$ partition points. and $11 n s$.

Fig. 4.7: Time-frequency analysis for example 2. 


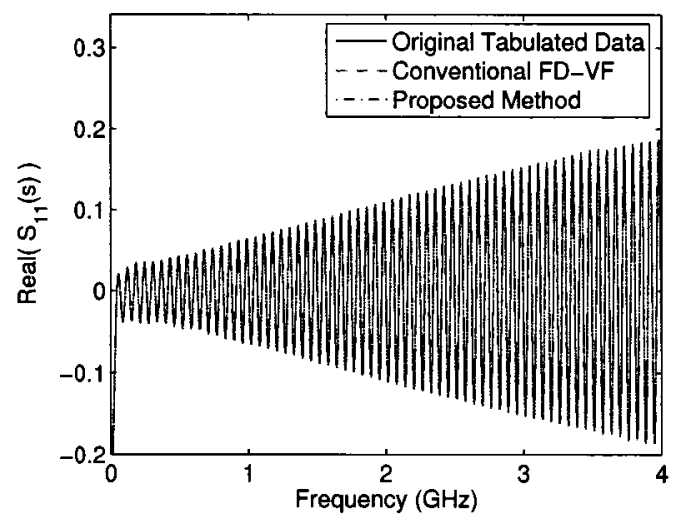

(a) Real part of $S_{11}(s)$.

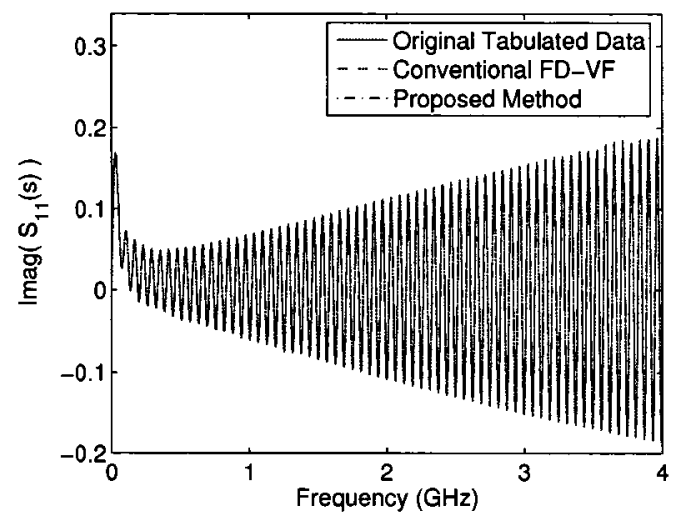

(c) Imaginary part of $S_{11}(s)$.

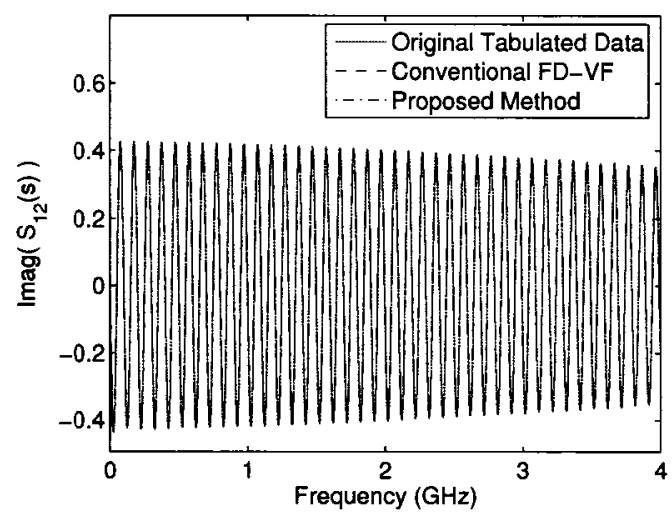

(e) Imaginary part of $S_{12}(s)$.

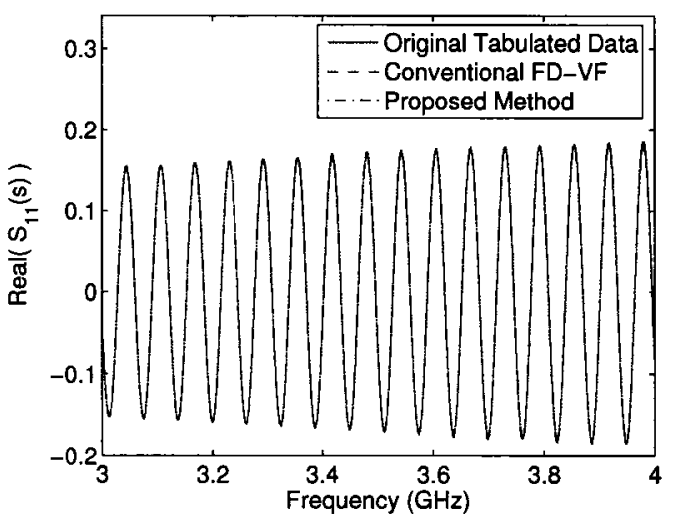

(b) An expanded view of Fig. 4.8(a).

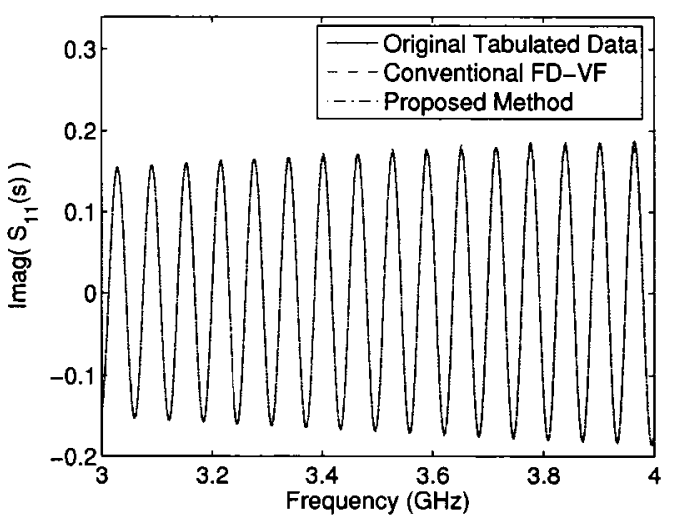

(d) An expanded view of Fig. 4.8(c).

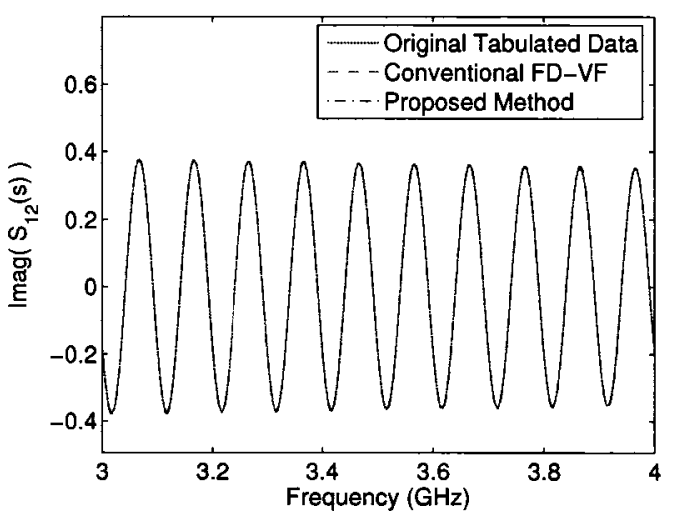

(f) An expanded view of Fig. 4.8(e).

Fig. 4.8: Frequency-domain comparisons for example 2. 


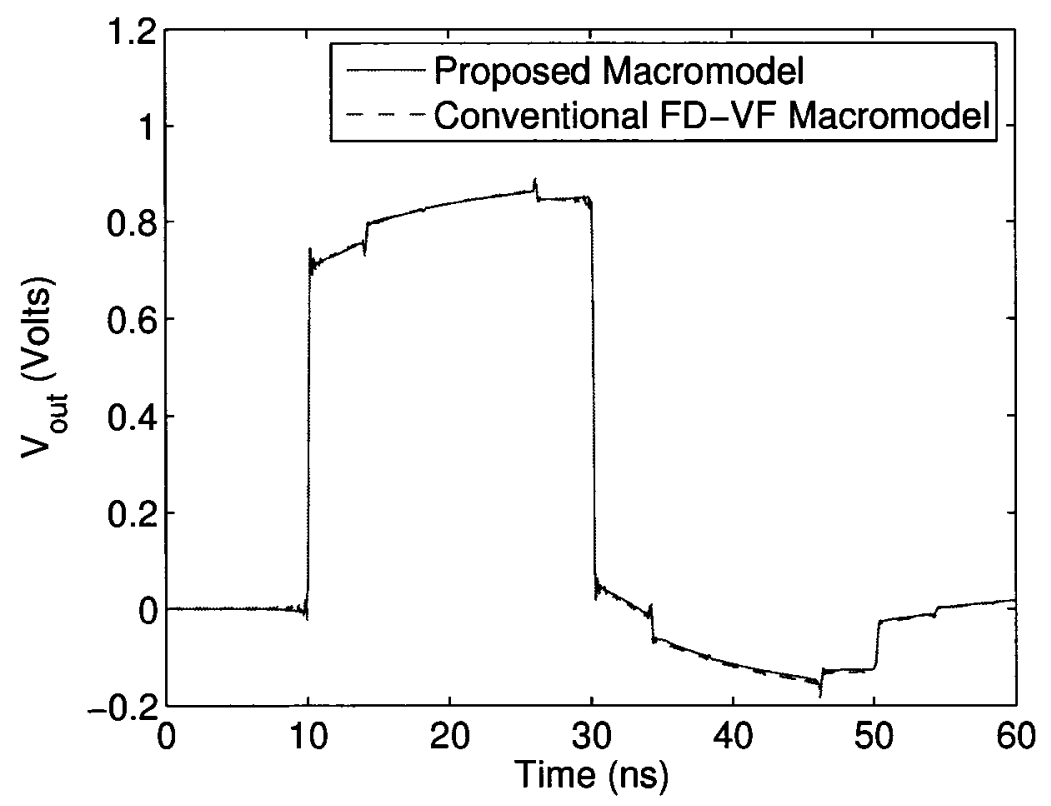

Fig. 4.9: Transient comparison for example 2.

algorithm required a total of 136 segments and 1004 poles to fit the entire network. In comparison, the FD-VF algorithm required 2640 poles to fit the entire network. Sample comparisons of the frequency-domain fittings are shown in Fig. 4.12.

Next, macromodels for both the proposed algorithm and FD-VF were simulated in HSPICE. Each model was excited by a $20 n s$ wide pulse with $0.1 n s$ rise/fall times. The proposed macromodel required 32.98 seconds to simulate in HSPICE. In comparison, the FD-VF macromodel required 117.36 seconds to simulate. The responses from both output ports are shown in Fig. 4.13. As seen, the results are in agreement. 


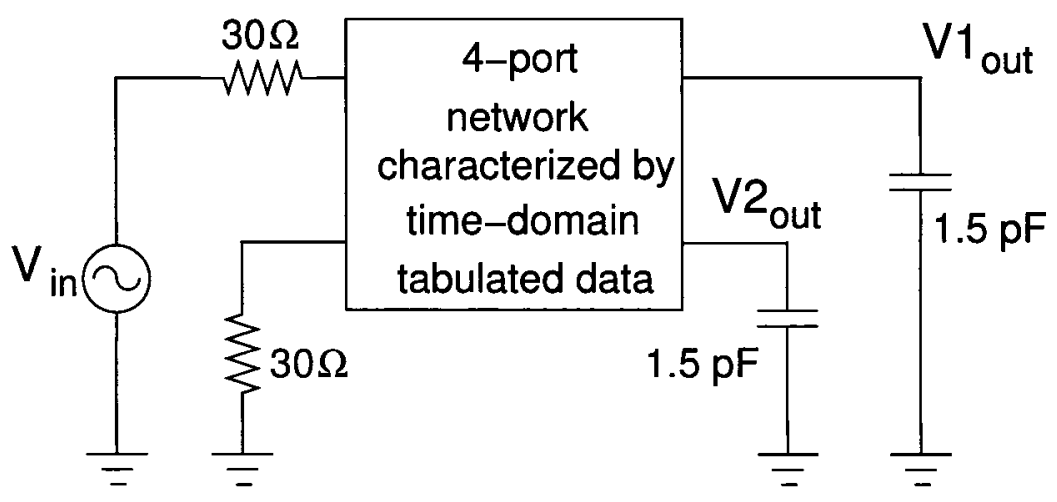

Fig. 4.10: Circuit for example 3.

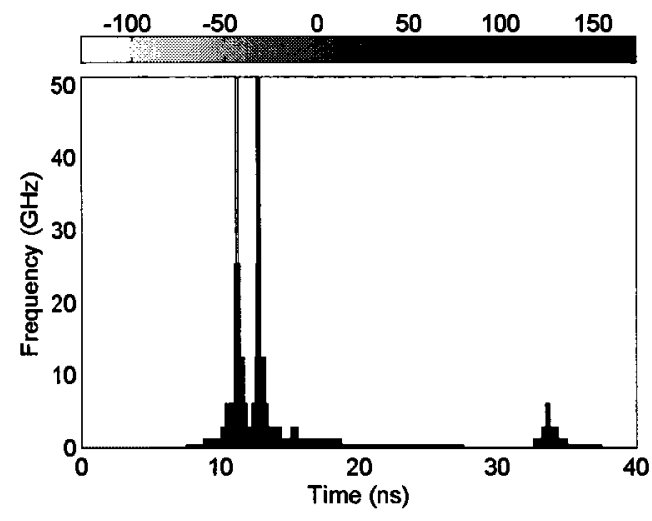

(a) Heisenberg tiles for wavelet decomposition.

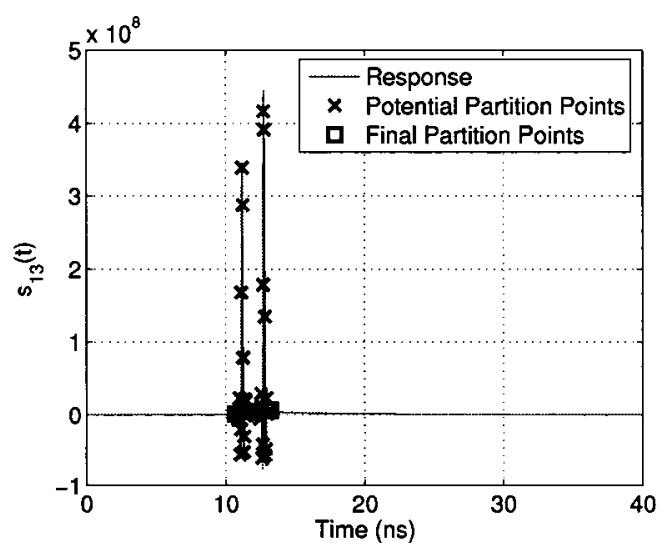

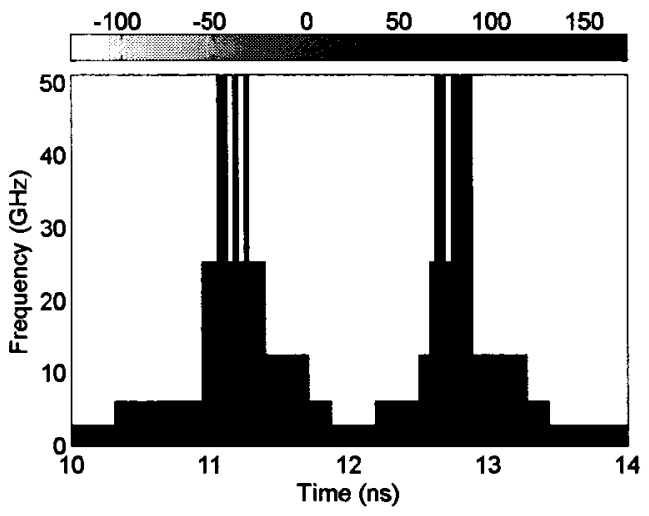

(b) An expanded view of Fig. 4.11(a) between $10 n s$ and $14 n s$.

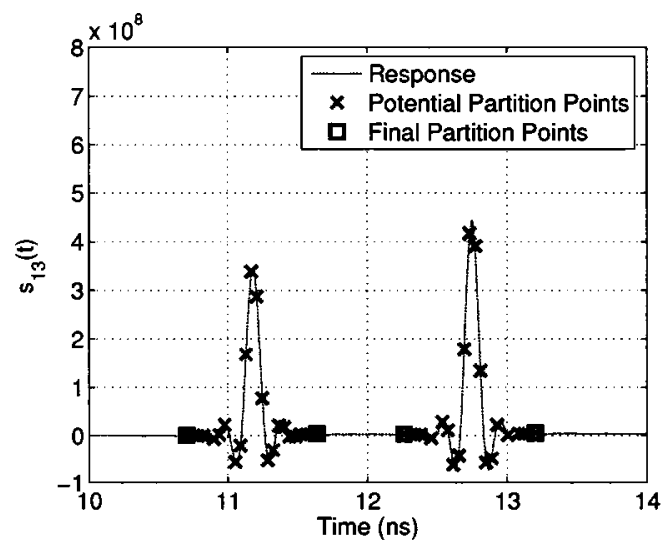

(c) Display of potential partition points and final (d) An expanded view of Fig. 4.11(c) between partition points. $10 n s$ and $14 n s$.

Fig. 4.11: Time-frequency analysis for example 3. 


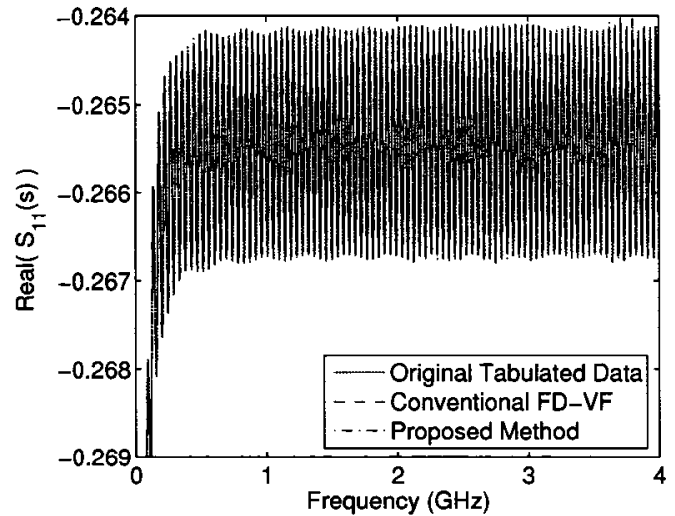

(a) Real part of $S_{11}(s)$.

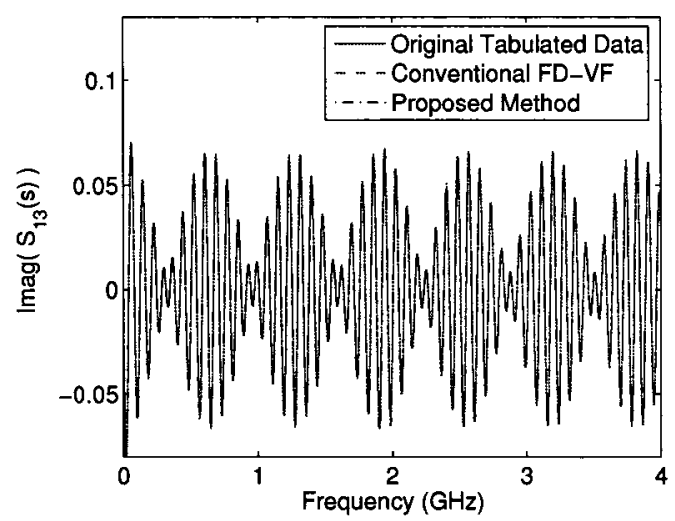

(c) Imaginary part of $S_{13}(s)$.

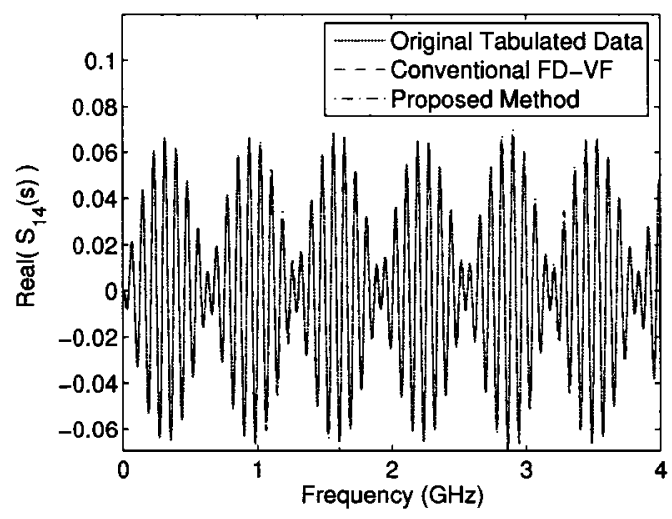

(e) Real part of $S_{14}(s)$.

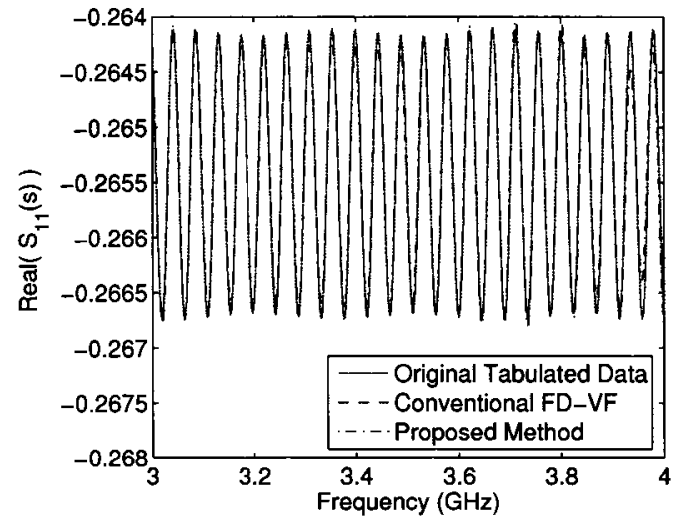

(b) An expanded view of Fig. 4.12(a).

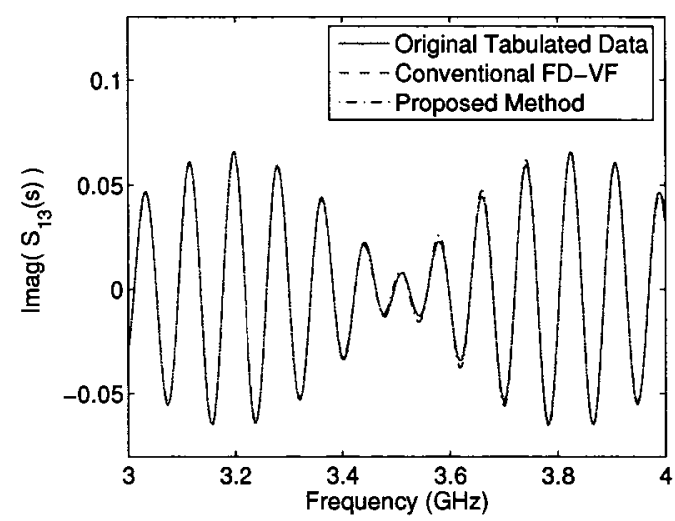

(d) An expanded view of Fig. 4.8(c).

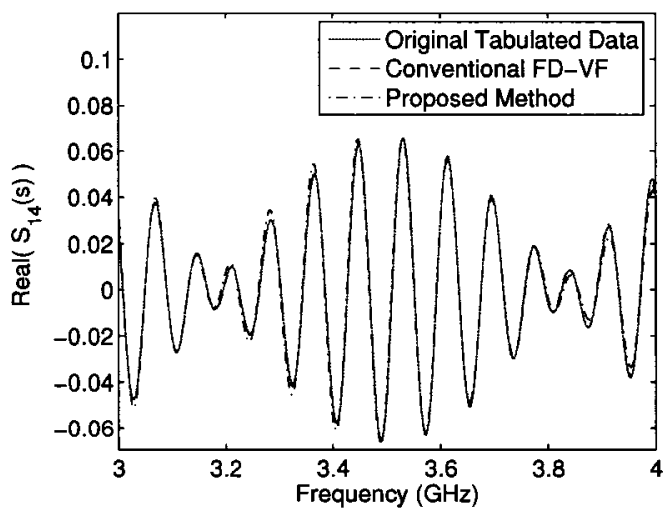

(f) An expanded view of Fig. 4.12(e).

Fig. 4.12: Frequency-domain comparisons for example 3. 


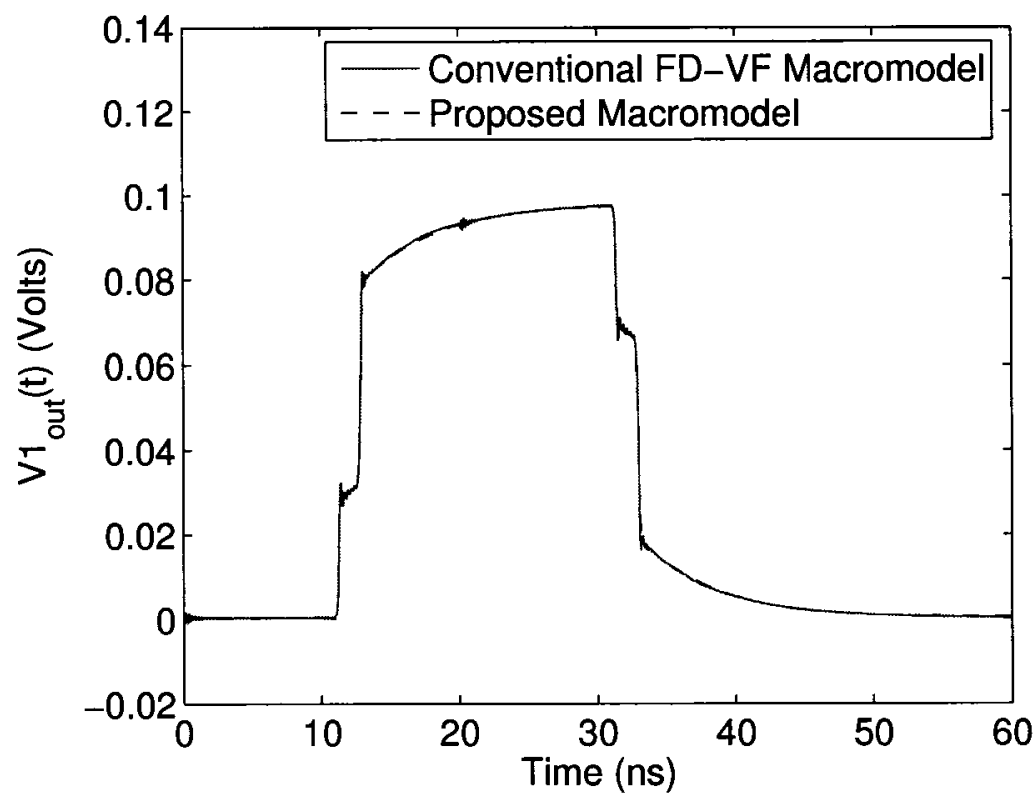

(a) Response of $V 1_{\text {out }}(t)$.

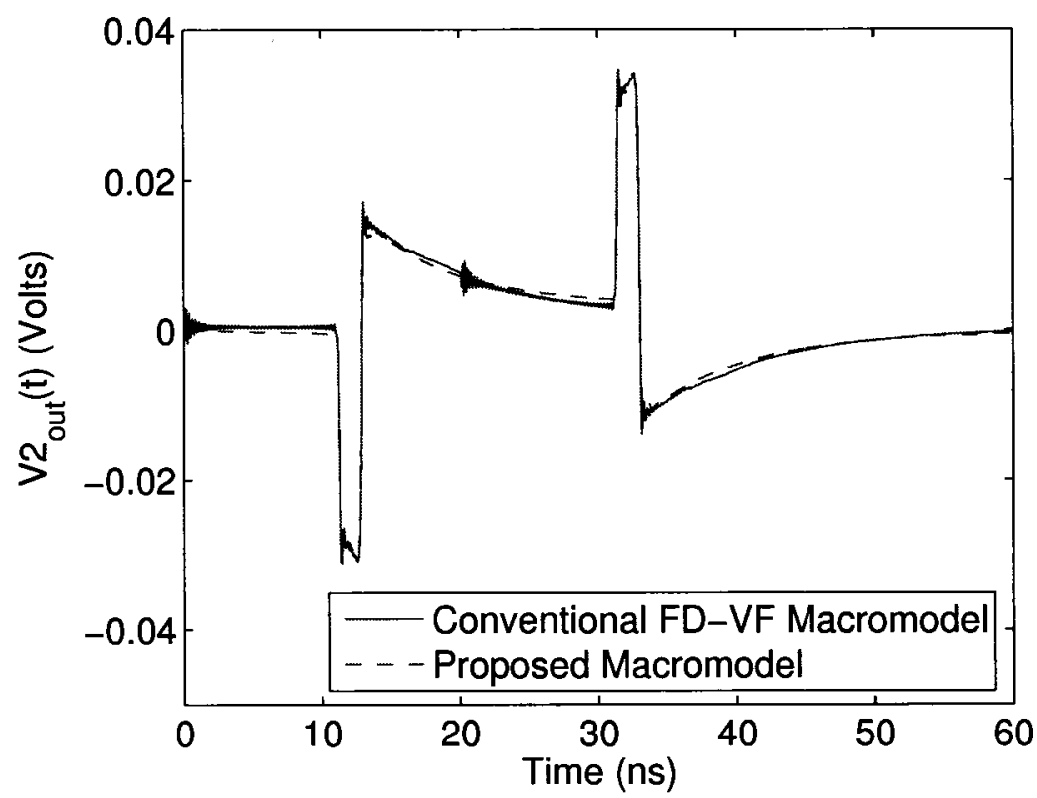

(b) Response of $V 2_{\text {out }}(t)$.

Fig. 4.13: Comparison of transient responses for example 3 . 


\subsection{Summary}

In this chapter, a new method has been presented for macromodeling tabulated data characterizing high-speed circuits with long delays. The new method partitions the time-domain impulse response using wavelet decomposition. Next, each partition is fit with a sum of delayed exponentials using Prony's algorithm to obtain a delayed rational function approximation. Methods presented in chapter 6 are used to convert this approximation into a DDE, and finally, a set of equivalent subcircuits for SPICE simulation. Based on the results achieved, this method can provide an improvement over the conventional FD-VF algorithm for obtaining highly compact macromodels for electrically long high-speed circuits characterized by tabulated data. 


\section{Chapter 5}

\section{Delay Extraction based Compact}

\section{Interconnect Macromodeling}

\section{Algorithm for Long Delay}

\section{Networks}

\subsection{Introduction}

In the previous chapter, a novel algorithm was developed for the construction of macromodels via delayed rational functions for networks characterized by tabulated data representing impulse port responses. However, time-domain impulse response data is not always available, and obtaining this data via the inverse fast Fourier transform (IFFT) can be noisy and error prone.

To overcome this difficulty, this chapter presents a novel low-order (compact) 84 
macromodeling algorithm for multiport long-delay networks characterized by tabulated time-domain port response data, $\{y(t)\}$, due to a corresponding input excitation, $\{x(t)\}$. The algorithm is based on partitioning the given time-domain port response data and subsequently, approximating each partition via delayed rational functions. The necessary theoretical foundations and implementation details are developed, followed by numerical examples to demonstrate the accuracy and efficiency of the proposed macromodel.

This chapter is organized as follows. Section 5.2 outlines the underlying concept and theory associated with the new algorithm. Next, section 5.3 gives a detailed development of the delayed rational function approximations and is followed by section 5.4, which discusses continuity enforcement in the delayed rational formulation. Section 5.5 presents wavelet decomposition based time-frequency analysis for the determination of partition points. Sections 5.6 and 5.7 present the numerical results and conclusions, respectively.

\subsection{Preliminaries and the Underlying Principle of the Proposed Algorithm}

In order to overcome the problem of high-order rational functions required for macromodeling long-delay networks characterized by time-domain tabulated data, a new approach based on delayed rational function approximations is given in this section. The algorithm first partitions the time-domain data into several intervals and then, approximates each interval as a delayed response to the input. The new method leads to highly compact (low-order) macromodels, which can be efficiently simulated using 
existing circuit simulators. Given the tabulated time-domain data, the objective of the new method is to produce delayed rational function approximation of the form

$$
H(s)=d_{0}+\sum_{i=0}^{N-1}\left(\sum_{m=1}^{M_{i}} \frac{R_{i, m}}{s-p_{i, m}}\right) e^{-s \tau_{s_{i}}}
$$

where $N$ is the number of partitions in the system. For the $i^{\text {th }}$ segment, $M_{i}$ is the total number of poles, $\left\{R_{i, m}\right\}$ and $\left\{p_{i, m}\right\}$ are the residues and poles, respectively, and $\tau_{s_{i}}$ is the delay. $d_{0}$ is the direct coupling constant for the $0^{\text {th }}$ segment.

To illustrate the concept and the advantages of the proposed methodology, consider the response of a lossy transmission line due to a triangular pulse shown in Fig. 5.1. This response is segmented into $N$ segments such that $\tau_{s_{0}}<\tau_{s_{1}}<\ldots<\tau_{s_{N}}$, ( $N=4$ in this example). The transfer function for the first interval, $\left[\tau_{s_{0}}, \tau_{s_{1}}\right]$, is shown in Fig. 5.2. Only 4 poles were required to fit this response (using the TDVF algorithm). Now consider the interval, $\left[\tau_{s_{0}}, \tau_{s_{2}}\right]$, which contains a single delayed reflection. The corresponding frequency-domain response is shown in Fig. 5.3 and in this case, 120 poles were required to approximate the response (a significantly large number compared to the previous case). The order of the approximation further increases if more reflections are introduced. Therefore, the objective of this algorithm is to take advantage of the fact that the responses with small delays and no reflections require very few poles to fit, compared to fitting the entire response directly.

The above inference can be extended even for the case of more complex (but practical) systems, where the delay in the system is long and the excitation pulse is short. In such cases, the response of the complete line can be partitioned into several delayed responses due to a single pulse. If the partitioning points are chosen such that only 


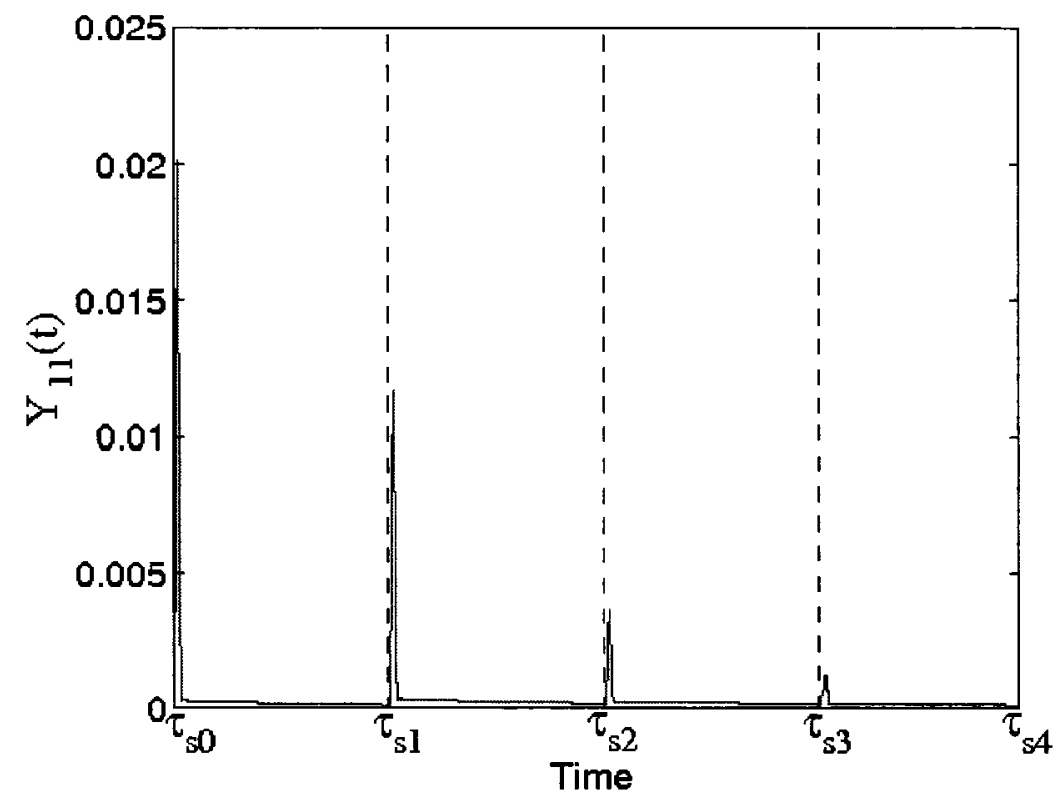

Fig. 5.1: Transient response for a lossy transmission line.

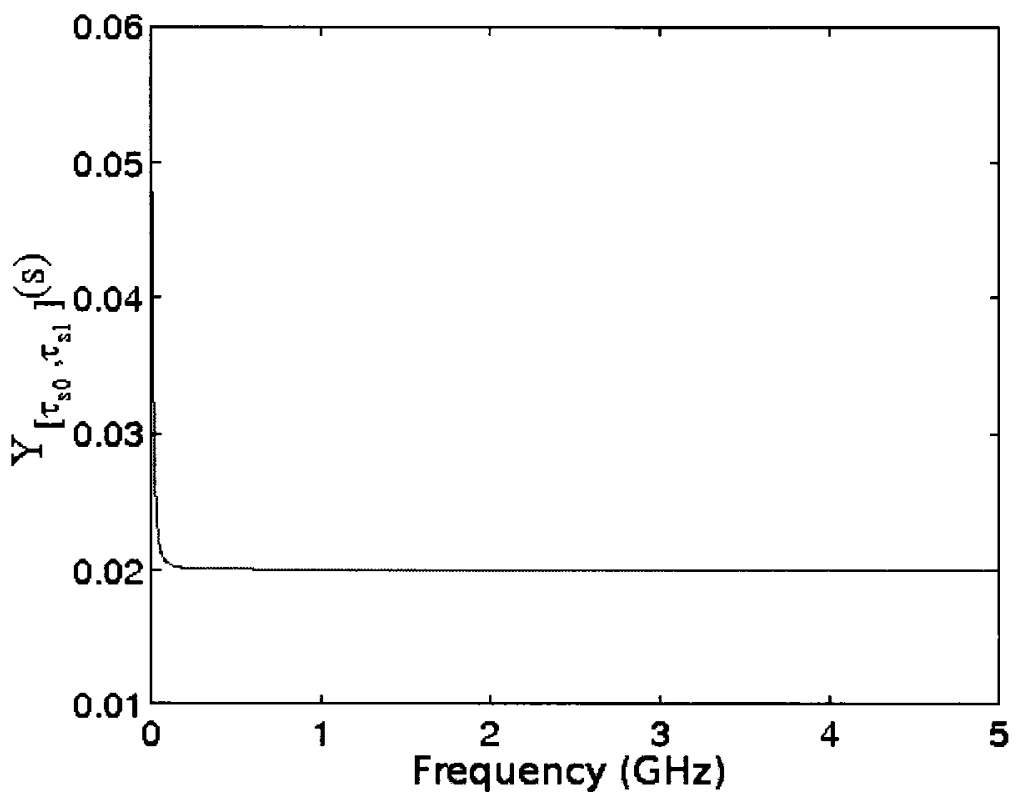

Fig. 5.2: Frequency response of the transfer function for interval $\left[\tau_{s_{0}}, \tau_{s_{1}}\right]$ of Fig. 5.1. 


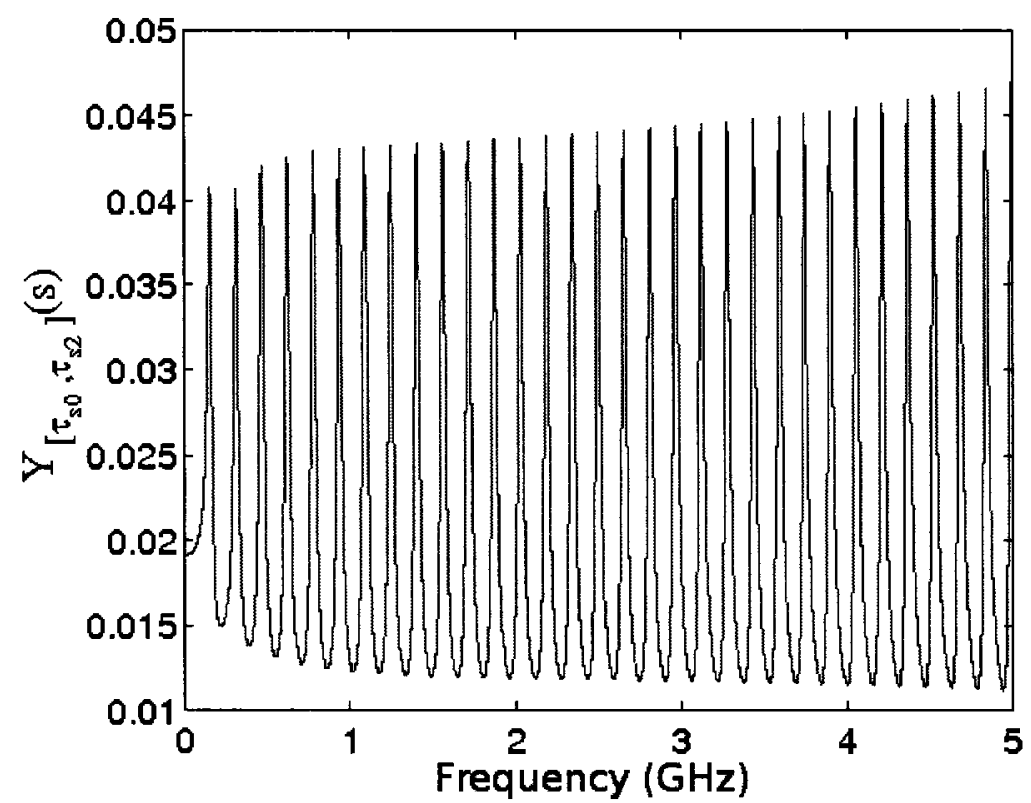

Fig. 5.3: Frequency response of the transfer function for interval $\left[\tau_{s_{0}}, \tau_{s_{2}}\right]$ of Fig. 5.1.

a single pulse response is contained in each time interval (i.e. the partitions contain no delayed responses), then the corresponding rational approximation of the data for each partition can be accomplished with significantly lower order rational approximations. The proposed algorithm explores the above inference while approximating the data with long delays.

The new algorithm begins by dividing the time-domain response data, $\{y(t)\}$, due to the input excitation, $\{x(t)\}$, into $N$ time intervals. Each of these intervals is considered as a separate delayed response to the input excitation. The transfer function for each of these intervals is found using (TD-VF) [36]. Next, the transfer function for the entire system is obtained as the sum of transfer functions due to each interval. This procedure results in a low-order delayed rational function, leading to a compact 
macromodel for SPICE simulation.

The development of the proposed algorithm is given in the next 3 sections, in the following order. First, the formulation of delayed rational transfer functions is presented. Next, the method used to enforce continuity between the segments is explained. Finally, the process used to determine the segmentation points is discussed.

\subsection{Formulation of Delayed Rational Functions}

In this section, a detailed formulation of the delayed rational functions is given. Assuming that the $N$ segments constituted by the partition points, $\tau_{s_{0}}<\tau_{s_{1}}<\ldots<\tau_{s_{N}}$, for the response, $\{y(t)\}$, have been determined (details are given in section 5.5 ), then the transfer function for the given system is approximated as a sum of delayed rational functions. Let $\{x(t)\}$ be the input function and let $h(t)$ be the impulse response (transfer function) relating $\{y(t)\}$ and $\{x(t)\}$ such that

$$
y(t)=h(t) * x(t)=\int_{0}^{t} h(\tau) x(t-\tau) d \tau
$$

where $*$ is the convolution operator. Next, the transfer function for the given system can be represented as

$$
h(t)=\sum_{i=0}^{N-1} h_{s_{i}}\left(t-\tau_{s_{i}}\right) u\left(t-\tau_{s_{i}}\right)=\sum_{i=0}^{N-1} g_{s_{i}}\left(t-\tau_{s_{i}}\right)
$$

where $\tau_{s_{0}}=0$ and

$$
g_{s_{i}}\left(t-\tau_{s_{i}}\right)=h_{s_{i}}\left(t-\tau_{s_{i}}\right) u\left(t-\tau_{s_{i}}\right)
$$


Let each transfer function, $h_{s_{i}}(t)$, be approximated as a sum of exponentials, $\hat{h}_{s_{i}}(t)$, in the form

$$
h_{s_{i}}(t) \approx \hat{h}_{s_{i}}(t)=d_{i} \delta(t)+\sum_{m=1}^{M_{i}} R_{i, m} e^{p_{i, m} t}
$$

where $M_{i}, R_{i, m}$, and $p_{i, m}$ are defined in (5.1). Then, the delayed approximation for each transfer function can be written as

$$
\hat{h}_{s_{i}}\left(t-\tau_{s_{i}}\right)=d_{i} \delta\left(t-\tau_{s_{i}}\right)+\sum_{m=1}^{M_{i}} R_{i, m} e^{p_{i, m}\left(t-\tau_{s_{i}}\right)}
$$

Using (5.3) and (5.6), the delayed approximation for the complete transfer function, $h(t)$, can now be written as

$$
\begin{aligned}
h(t) & \approx \sum_{i=0}^{N-1} \hat{h}_{s_{i}}\left(t-\tau_{s_{i}}\right) u\left(t-\tau_{s_{i}}\right) \\
& =d_{0} \delta(t)+\sum_{i=0}^{N-1} \sum_{m=1}^{M_{i}} R_{i, m} e^{p_{i, m}\left(t-\tau_{s_{i}}\right)} u\left(t-\tau_{s_{i}}\right) .
\end{aligned}
$$

The Laplace transform of (5.7) yields the formulation in (5.1), which is a delayed rational function approximation for the system transfer function in the Laplace-domain.

Next, the details of computing an approximation for each $\hat{h}_{s_{i}}(t)$ in the form of (5.5) are given. The process involved in computing $\hat{h}_{s_{i}}(t)$ (the rational approximation for a particular transient partition) is explained as follows. To begin, we consider data on the time interval $\left[\tau_{s_{0}}, \tau_{s_{1}}\right]$ and obtain $\hat{h}_{s_{0}}(t)$. Next, we generalize this process to obtain $\hat{h}_{s_{i}}(t)$ for any segment $i$.

Computation of $\hat{\mathbf{h}}_{\mathbf{s}_{\mathbf{0}}}(\mathbf{t})$ : Consider the data on the interval $t \in\left[\tau_{s_{0}}, \tau_{s_{1}}\right]$ shown in Fig. 5.4. The approximation of $h_{s_{0}}(t)$ is found by using the TD-VF algorithm and 
considering the response and input data sets over the duration $t \in\left[\tau_{s_{0}}, \tau_{s_{1}}\right]$. For this purpose, the following lemma is used.

Lemma 1. Given

$$
\begin{array}{ll}
y_{s_{0}}(t)=y(t), & t \in\left[\tau_{s_{0}}, \tau_{s_{1}}\right], \\
x_{s_{0}}(t)=x(t), & t \in\left[\tau_{s_{0}}, \tau_{s_{1}}\right],
\end{array}
$$

we have

$$
y_{s_{0}}(t)=h_{s_{0}}(t) * x_{s_{0}}(t), \quad t \in\left[\tau_{s_{0}}, \tau_{s_{1}}\right]
$$

Proof. Using (5.3), (5.2) can be expressed as

$$
\begin{aligned}
y(t) & =\sum_{i=0}^{N-1} g_{s_{i}}\left(t-\tau_{s_{i}}\right) * x(t) \\
& =\int_{0}^{t} \sum_{i=0}^{N-1} g_{s_{i}}\left(\tau-\tau_{s_{i}}\right) x(t-\tau) d \tau .
\end{aligned}
$$

Since the considered interval is $t \in\left[\tau_{s_{0}}, \tau_{s_{1}}\right]$ (with $\tau_{s_{0}}=0$ ), the functions $g_{s_{i}}\left(t-\tau_{s_{i}}\right.$ ) all vanish on this interval except $g_{s_{0}}\left(t-\tau_{s_{0}}\right)$. Therefore,

$$
\begin{aligned}
y_{s_{0}}(t) & =\int_{0}^{t} g_{s_{0}}\left(\tau-\tau_{s_{0}}\right) x_{s_{0}}(t-\tau) d \tau \\
& =g_{s_{0}}\left(t-\tau_{s_{0}}\right) * x_{s_{0}}(t) \\
& =h_{s_{0}}(t) * x_{s_{0}}(t)
\end{aligned}
$$

It is clear from Lemma 1 that the TD-VF algorithm can be applied to the data, 


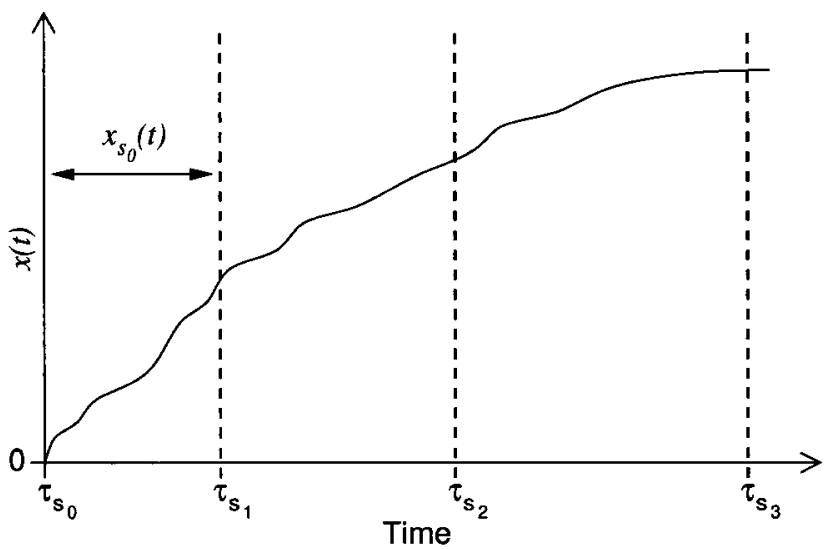

(a)

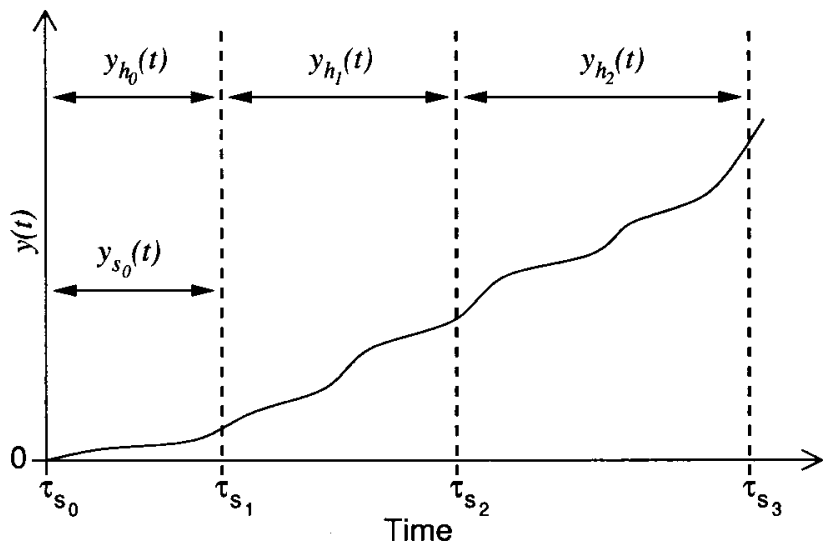

(b)

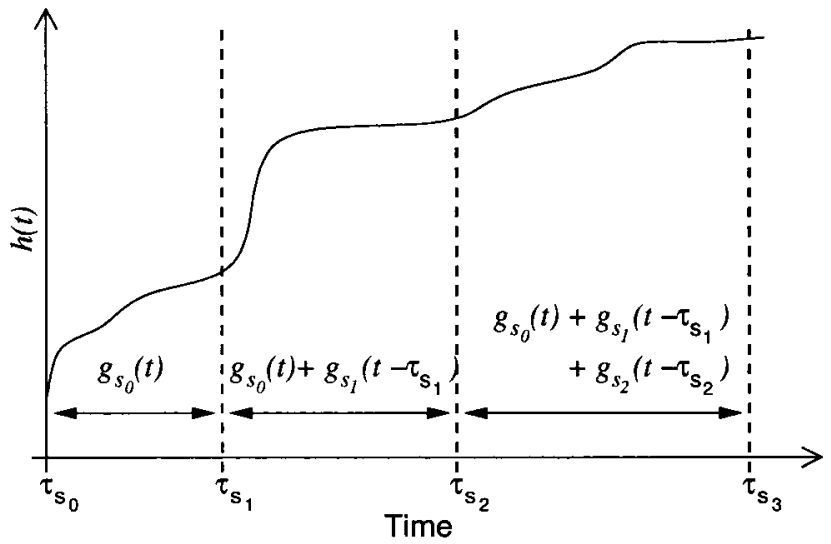

(c)

Fig. 5.4: Illustrative graphs showing time-domain data and partition points for fitting of first segment. 
$\left\{y_{s_{0}}(t)\right\}$ and $\left\{x_{s_{0}}(t)\right\}$, on the interval $\left[\tau_{s_{0}}, \tau_{s_{1}}\right]$ to obtain the approximate transfer function, $\hat{h}_{s_{0}}(t)$, for $h_{s_{0}}(t)$ in the form of (5.5). Note that $\hat{h}_{s_{0}}(t)$ as well as the corresponding response $\hat{y}_{s_{0}}(t)$ are defined over the interval $t \in[0, \infty]$.

Computation of $\hat{\mathbf{h}}_{\mathbf{s}_{\mathbf{i}}}(\mathbf{t}), \mathbf{i} \in\{\mathbf{1}, \mathbf{2}, \ldots, \mathbf{N}-\mathbf{1}\}$ : To compute $\hat{h}_{s_{1}}(t)$, TD-VF is applied using $y_{s_{1}}(t)=\left\{y_{h_{i}}(t)-\hat{y}_{s_{0}}(t)\right\}$ as the response data and $x_{s_{1}}(t)=\left\{x\left(t-\tau_{s_{1}}\right) u(t-\right.$ $\left.\left.\tau_{s_{1}}\right)\right\}$ as the input function for the interval $t \in\left[\tau_{s_{1}}, \tau_{s_{2}}\right]$ (see Fig. 5.5 for an illustration). The result is the approximation $\hat{h}_{s_{1}}(t)$ defined on $t \in[0, \infty]$. The following lemma is used to identify appropriate response and input vectors for TD-VF based approximations, so as to recursively construct the $\hat{h}_{s_{i}}(t)$ for any $i \in\{1,2, \ldots, N-1\}$.

Lemma 2. Given the $i^{\text {th }}$ segment with the following definitions,

$$
\begin{array}{lrl}
y_{h_{i}}(t)=y(t), & t \in\left[\tau_{s_{i}}, \tau_{s_{i+1}}\right], \\
x_{s_{i}}(t)=x\left(t-\tau_{s_{i}}\right) u\left(t-\tau_{s_{i}}\right), & t \in\left[\tau_{s_{i}}, \tau_{s_{i+1}}\right], \\
\hat{y}_{s_{j}}(t)=\hat{g}_{s_{j}}\left(t-\tau_{s_{j}}\right) * x(t) \approx y_{s_{j}}(t), & t \in[0, \infty],
\end{array}
$$

we have

$$
y_{s_{i}}(t)=h_{s_{i}}(t) * x_{s_{i}}(t) \approx y_{h_{i}}(t)-\sum_{j=0}^{i-1} \hat{y}_{s_{j}}(t), \quad t \in\left[\tau_{s_{i}}, \tau_{s_{i+1}}\right]
$$

Proof. From (5.11), we have

$$
y(t)=\sum_{j=0}^{i} g_{s_{j}}\left(t-\tau_{s_{j}}\right) * x(t)+\sum_{j=i+1}^{N-1} g_{s_{j}}\left(t-\tau_{s_{j}}\right) * x(t)
$$

where $i$ corresponds to the $i^{t h}$ segment. Since $t \in\left[\tau_{s_{i}}, \tau_{s_{i+1}}\right]$, the functions $g_{s_{j}}\left(t-\tau_{s_{j}}\right)$ all vanish on the $i^{\text {th }}$ interval for $j>i$. Therefore, (5.11) can be written using the 


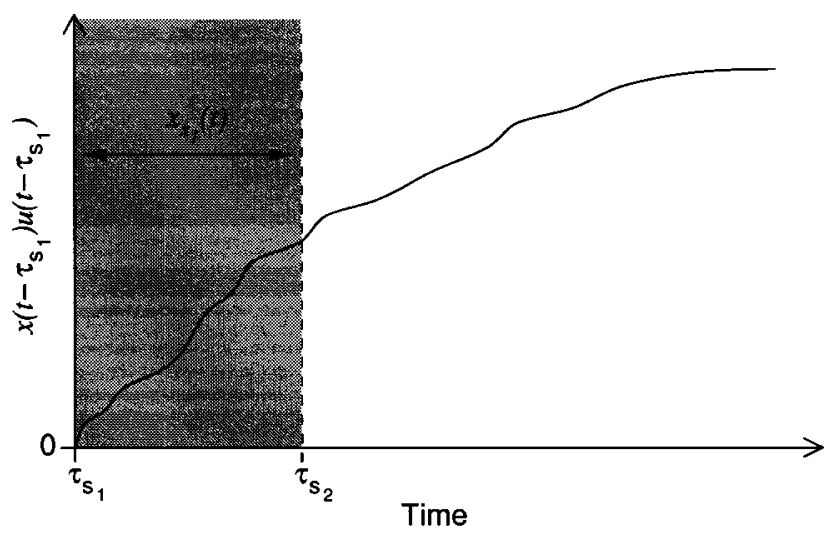

(a)

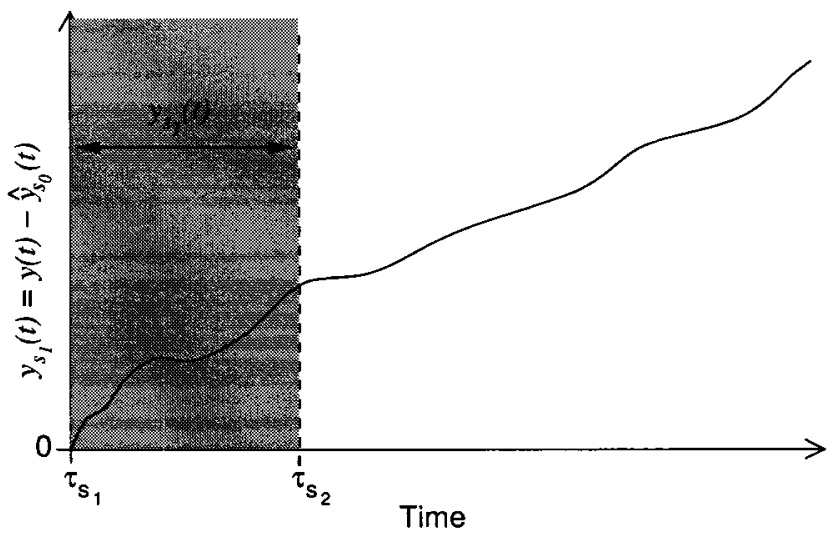

(b)

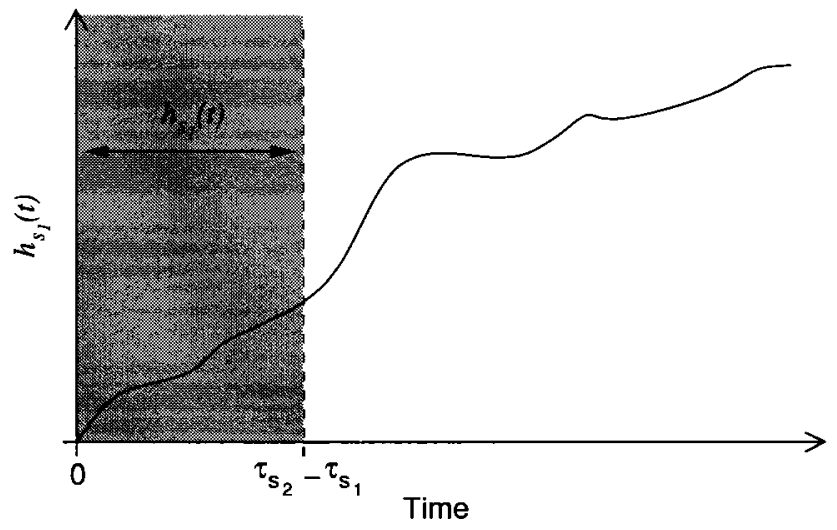

(c)

Fig. 5.5: Illustrative graphs showing time-domain data and partition points for fitting of second segment. 
definitions in (5.13) as

$$
\begin{aligned}
y_{h_{i}}(t) & =\sum_{j=0}^{i-1} g_{s_{j}}\left(t-\tau_{s_{j}}\right) * x(t)+g_{s_{i}}\left(t-\tau_{s_{i}}\right) * x(t) \\
& =\sum_{j=0}^{i-1} y_{s_{j}}(t)+g_{s_{i}}\left(t-\tau_{s_{i}}\right) * x(t) \\
& \approx \sum_{j=0}^{i-1} \hat{y}_{s_{j}}(t)+g_{s_{i}}\left(t-\tau_{s_{i}}\right) * x(t) .
\end{aligned}
$$

Rearranging (5.16) yields

$$
g_{s_{i}}\left(t-\tau_{s_{i}}\right) * x(t) \approx y_{h_{i}}(t)-\sum_{j=0}^{i-1} \hat{y}_{s_{j}}(t)
$$

or

$$
y_{s_{i}}(t) \approx y_{h_{i}}(t)-\sum_{j=0}^{i-1} \hat{y}_{s_{j}}(t) .
$$

Next, noting the LHS of (5.17) is a delayed function, convolved with another function, it can be written in the Laplace-domain and rearranged using (5.4) to obtain

$$
\begin{aligned}
\mathfrak{L}\left\{\left[g_{s_{i}}\left(t-\tau_{s_{i}}\right)\right] * x(t)\right\} & =\mathfrak{L}\left\{\left[h_{s_{i}}\left(t-\tau_{s_{i}}\right) u\left(t-\tau_{s_{i}}\right)\right] * x(t)\right\} \\
& =H_{s_{i}}(s) e^{-s \tau_{s_{i}}} X(s) \\
& =\left[H_{s_{i}}(s)\right]\left[e^{-s \tau_{s_{i}}} X(s)\right] \\
& =\mathfrak{L}\left\{h_{s_{i}}(t) *\left[x\left(t-\tau_{s_{i}}\right) u\left(t-\tau_{s_{i}}\right)\right]\right\}
\end{aligned}
$$

where $H_{s_{i}}(s)$ and $X(s)$ are the Laplace-domain representations of $h_{s_{i}}(t)$ and $x(t)$, 
respectively. It is clear that using (5.19) and (5.17), (5.18) can be written as

$$
y_{s_{i}}(t)=h_{s_{i}}(t) *\left\{x\left(t-\tau_{s_{i}}\right) u\left(t-\tau_{s_{i}}\right)\right\} \approx y_{h_{i}}(t)-\sum_{j=0}^{i-1} \hat{y}_{s_{j}}(t)
$$

From (5.20), it is apparent that TD-VF can be applied recursively to each of the remaining intervals, to obtain the approximation for the $i^{\text {th }}$ transfer function, $\hat{h}_{s_{i}}(t)$, using $y_{s_{i}}(t) \approx\left\{y_{h_{i}}(t)-\sum_{j=0}^{i-1} \hat{y}_{s_{j}}(t)\right\}$ as the response data and $x_{s_{i}}(t)=\left\{x\left(t-\tau_{s_{i}}\right) u(t-\right.$ $\left.\left.\tau_{s_{i}}\right)\right\}$ as the input data for the interval $t \in\left[\tau_{s_{i}}, \tau_{s_{i+1}}\right]$. Once each of the $\hat{h}_{s_{i}}(t)$ is obtained as a sum of exponentials, they are used to obtain an approximation for the complete transfer function, $h(t)$, as in (5.7).

It is to be noted that using TD-VF based approximation for the data in a given partition helps in obtaining an accurate and continuous approximation within the interval. However, it does not ensure the continuity of the approximation at the boundaries of the interval. Hence, to enforce the continuity at the boundary points of the intervals, details are given in the next section.

\subsection{Continuity Enforcement}

It is desired to have the transfer function, $h(t)$, continuous at each of the partition points to ensure there are no discontinuities in the response $y(t)=h(t) * x(t)$ (if $h(t)$ is not continuous, $y(t)$ is not guaranteed to be continuous). By definition, a function, $f$, is continuous at $t=a$ if the following conditions hold [65].

1. $f$ is defined at $t=a$ (i.e. $f(a)$ is finite), 
2. $\lim _{t \rightarrow a^{+}} f(t)=\lim _{t \rightarrow a^{-}} f(t)=f(a)$.

Therefore, in order to ensure continuity at the $i^{\text {th }}$ partition point, $\tau_{s_{i}}$, it is required that the above conditions hold for $h_{s_{i}}(t)$. Using (5.3) and (5.4), this translates into

$$
h\left(\tau_{s_{i}}\right)=\lim _{t \rightarrow \tau_{s_{i}}^{-}} \sum_{j=0}^{N-1} g_{s_{j}}\left(t-\tau_{s_{j}}\right)=\lim _{t \rightarrow \tau_{s_{i}}^{+}} \sum_{j=0}^{N-1} g_{s_{j}}\left(t-\tau_{s_{j}}\right)
$$

or

$$
\lim _{t \rightarrow \tau_{s_{i}}^{-}} \sum_{j=0}^{i-1} g_{s_{j}}\left(t-\tau_{s_{j}}\right)=\lim _{t \rightarrow \tau_{s_{i}}^{+}} \sum_{j=0}^{i} g_{s_{j}}\left(t-\tau_{s_{j}}\right) .
$$

If each $g_{s_{j}}\left(t-\tau_{s_{j}}\right)$ is continuous at $t=\tau_{s_{i}}$ then

$$
\lim _{t \rightarrow \tau_{s_{i}}^{-}} g_{s_{j}}\left(t-\tau_{s_{j}}\right)=\lim _{t \rightarrow \tau_{s_{i}}^{+}} g_{s_{j}}\left(t-\tau_{s_{j}}\right)
$$

holds for $j<i$. Using (5.23) in (5.22) yields

$$
\begin{aligned}
\lim _{t \rightarrow \tau_{s_{i}}^{+}} g_{s_{i}}\left(t-\tau_{s_{i}}\right) & =h_{s_{i}}\left(\tau_{s_{i}}-\tau_{s_{i}}\right) u\left(\tau_{s_{i}}-\tau_{s_{i}}\right)=h_{s_{i}}(0) \\
& =0
\end{aligned}
$$

Therefore, in order for the transfer function to be continuous at the partition points, it is required that $h_{s_{i}}(t=0)=0$.

This is accomplished by fixing one of the residues in the residue identification process of the TD-VF algorithm. To enforce $h_{s_{i}}(0)=0$ for all $i \neq 0$, set

$$
h_{s_{i}}(0)=d_{i} \delta(0)+\sum_{m=1}^{M_{i}} R_{i, m} e^{p_{i, m}(0)}=0
$$


It is clear that $d_{i}=0$ ensures that the delta function vanishes. Then,

$$
\sum_{m=1}^{M_{i}} R_{i, m}=0
$$

Fix $R_{i, 1}$ and rearrange (5.26) to obtain the relation,

$$
R_{i, 1}=-\sum_{m=2}^{M_{i}} R_{i, m}
$$

Next, applying the standard least squares residue identification problem (in TD-VF) we have

$$
y(t)=\sum_{m=1}^{M_{i}} R_{i, m} x_{i, m}(t)
$$

where $x_{i, m}(t)$ is defined as in (2.51) for the $i^{\text {th }}$ segment. Using (5.27) and rearranging (5.28) we have

$$
y(t)=\left(-\sum_{m=2}^{M_{i}} R_{i, m}\right) x_{i, 1}(t)+\sum_{m=2}^{M_{i}} R_{i, m} x_{i, m}(t)
$$

or

$$
y(t)=\sum_{m=2}^{M_{i}} R_{i, m}\left(x_{i, m}(t)-x_{i, 1}(t)\right) .
$$

Equation (5.30) can be solved as a least squares problem for the residues $\left\{R_{i, m}\right\}$ for $m=\left\{2, \ldots, M_{i}\right\}$, which are then used in (5.27) to find $R_{i, 1}$.

The next section describes the details of the proposed algorithm for determining partitioning points, which are required by the delayed rational function formulation presented in section 5.3 and 5.4 . 


\subsection{Determination of Partition Points}

As discussed in the introduction and section 5.2, it is clear that if each partition consists of a single response with no delay, then the corresponding approximation of the data in that interval can be achieved with lower-order rational functions. In addition, the proposed algorithm exploits the fact that each delay in the data corresponds to a region of high frequency content in the time-domain response. Consequently, in the proposed algorithm, the interval (or split) points are determined by performing a time-frequency analysis of the time-domain data using wavelet decomposition [63]. Wavelet based time-frequency analysis provides information on a function, $y(t)$, in both the frequency-domain and time-domain, allowing for accurate localization of high frequency content in the time-domain.

In chapter 4, wavelet decomposition was used to identify the partition points on the time-domain impulse response data. However, in this case, the goal is to determine the partition points of the response data, $\{y(t)\}$, from the input excitation, $\{x(t)\}$. We proceed as in section 4.3 to determine the potential partition points. However, in order to determine the final partition points, a new method is developed (since the data now represents a response to a pulse function, not the impulse response). In this method, the first point in each cluster is chosen as the partition point (to avoid including any reflected wave in the considered partition). A detailed discussion of this process is given in section 5.6.

A summary of the steps involved in the proposed algorithm for the macromodeling of long delay networks is presented (in the form of pseudocode) in Algorithm 1. 
Algorithm 1 Pseudocode for the proposed algorithm

Step 1: Obtain tabulated time-domain response data, $\{y(t)\}$, to the input data, $\{x(t)\}$, at time points $t=\left\{0, t_{1}, \ldots, t_{\max }\right\}$.

Step 2: Determine partition points, $\tau_{s_{0}}<\tau_{s_{1}}<\ldots<\tau_{s_{N}}$, by performing wavelet decomposition on the response data, $\{y(t)\}$, as discussed in section 5.5 .

Step 3: Apply TD-VF (using continuity enforcement as discussed in section 5.4) on the response, $\{y(t)\}$, to the input excitation, $\{x(t)\}$, using the data from the interval $t \in\left[\tau_{s_{0}}, \tau_{s_{1}}\right]$. This yields the transfer function $\hat{h}_{s_{0}}(t)$.

\section{Step 4:}

for $i=1: N-1$ do

a) Calculate the data set $\left\{y_{s_{i}}(t)\right\}=\left\{y_{h_{i}}(t)-\sum_{j=0}^{i-1} \hat{y}_{s_{j}}(t)\right\}$ for $t \in\left[\tau_{s_{i}}, \tau_{s_{i+1}}\right]$ with $\hat{y}_{s_{j}}(t)=\hat{g}_{s_{j}}\left(t-\tau_{s_{i}}\right) * x(t)$.

b) Apply TD-VF (with continuity enforcement on the last point) using $\left\{y_{s_{i}}(t)\right\}=$ $\left\{y_{h_{i}}(t)-\sum_{j=0}^{i-1} \hat{y}_{s_{j}}(t)\right\}$ as the response data and $\left\{x_{s_{i}}(t)\right\}=\left\{x\left(t-\tau_{s_{i}}\right) u\left(t-\tau_{s_{i}}\right)\right\}$ as the input data for $t \in\left[\tau_{s_{i}}, \tau_{s_{i+1}}\right]$. This yields the transfer function $\hat{h}_{s_{i}}(t)$. end for

Step 5: Construct the complete transfer function, $h(t)$, and subsequently, $H(s)$, using (5.7) and (5.1), respectively. 
TABLE 5.1: A summary of the CPU performance of the macromodels.

\begin{tabular}{|l|c|c|c|}
\hline & $\begin{array}{c}\text { Proposed } \\
\text { (seconds) }\end{array}$ & $\begin{array}{c}\text { Conventional TD-VF } \\
\text { (seconds) }\end{array}$ & $\begin{array}{c}\text { Speed-up of } \\
\text { Proposed }\end{array}$ \\
\hline \hline Example 1 & 4.03 & 215.76 & $\mathbf{5 4}$ \\
\hline Example 2 & 16.87 & 356.81 & $\mathbf{2 1}$ \\
\hline Example 3 & 3.32 & 107.98 & $\mathbf{3 3}$ \\
\hline
\end{tabular}

\subsection{Numerical Results}

This section presents several numerical examples assessing the accuracy and efficiency of the proposed algorithm. Comparisons are made between the proposed algorithm and the conventional TD-VF algorithm for several networks. In addition, the proposed macromodel is realized using techniques discussed in chapter 6 for transient SPICE comparisons with the TD-VF macromodel. Example 1 tests the proposed algorithm on the time-domain admittance parameters of a two-port transmission line and compares both results to the HSPICE W-Element. Example 2 tests the algorithm on the time-domain admittance parameters of an unknown two-port network. Next, example 3 considers a four-port structure described by time-domain admittance parameters. A summary of the results is shown in Table 5.1. Transient simulations were performed using HSPICE on an AMD Athlon $1.83 \mathrm{GHz}$ CPU.

\subsubsection{Example 1}

In order to validate the proof of concept for the proposed algorithm, an interconnect circuit with known model parameters is selected (Fig. 5.6). To validate the accuracy of the proposed algorithm, the interconnect is characterized at its ports with the timedomain tabulated data and the proposed macromodel is simulated using HSPICE. 
The resulting transient response of the network is compared against the transient response by replacing the macromodel with the W-element model (of HSPICE) for the interconnect.

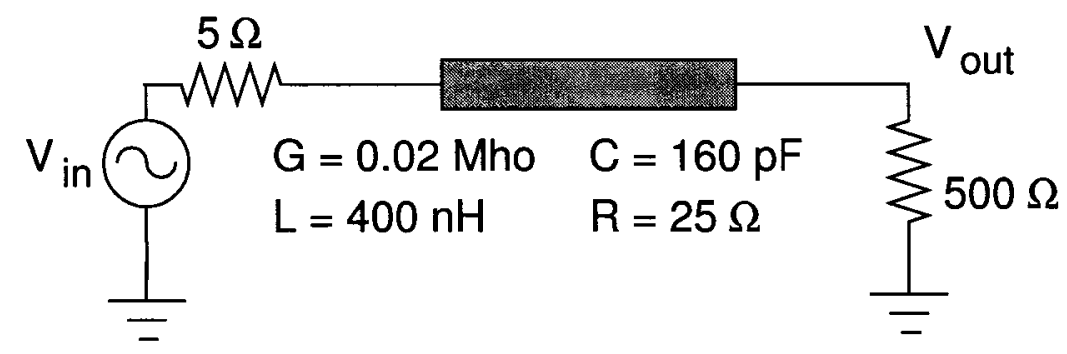

Fig. 5.6: Circuit for example 1.

The time-domain tabulated data for $y_{11}(t)$, as well as its time-frequency analysis are shown in Fig. 5.7. In this example, to determine the partitioning points, the forward wavelet transform was applied using orthogonal Daubechies wavelets with four filter coefficients [63], [64]. The resulting wavelet decomposition (in the form of Heisenberg tiles [63] on the time-frequency plane) shown in Fig. 5.7(a) corresponding to the time-domain response of an electrically long interconnect in Fig. 5.7(c). For clarity, an expanded view of Fig. 5.7(a) and Fig. 5.7(c) between 15.5ns and 16.5ns is given in Fig. 5.7(b) and Fig. 5.7(d), respectively. Wavelet coefficients with larger magnitudes and at higher frequency scales are selected as potential splitting points. This information is extracted and the potential split points are shown using ' $x$ ' in Figs. 5.7(c) and 5.7(d), which tend to occur in clusters. To determine the actual split points from the potential split points, clusters of points in regions about $4-5$ times the pulse width of the input excitation (which is significantly shorter than the associated delays of this example and the input is a triangular pulse with a pulse width of $0.2 n s$ ) were isolated. The first point in each cluster was chosen as the partition 
point (to avoid including any reflected wave in the considered partition). The selected partition points for this example are shown by ' $\square$ ' in Fig. 5.7(c) and Fig. 5.7(d).

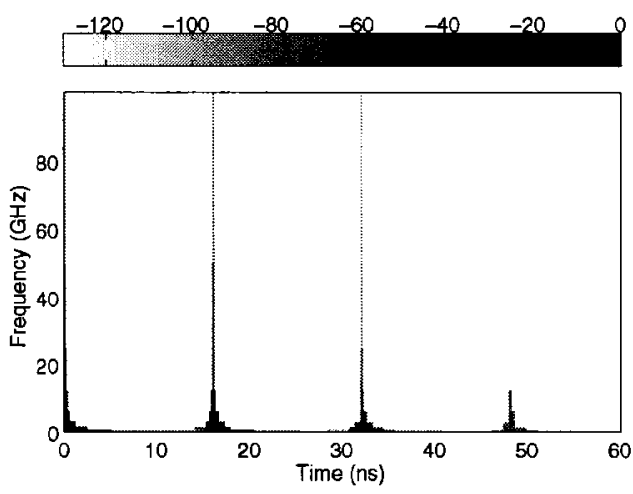

(a) Heisenberg tiles for wavelet decomposition.

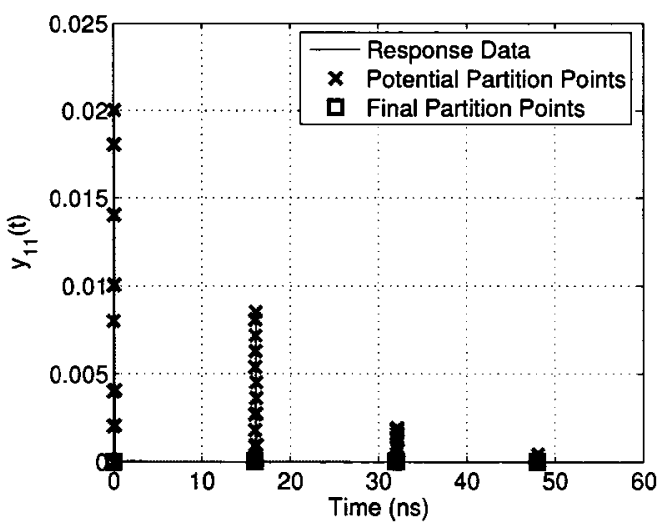

(c) Display of potential partition points and final (d) partition points.

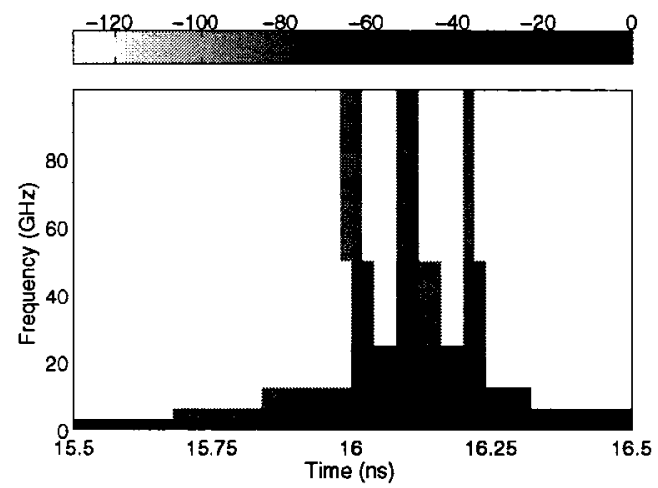

(b) An expanded view of Fig. 5.7(a) between $15.5 n s$ and $16.5 n s$.

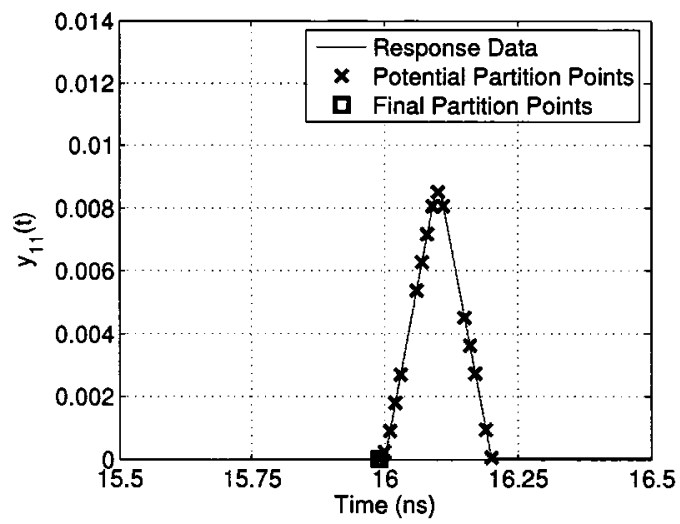

(d) An expanded view of Fig. 5.7(c) between $15.5 n s$ and $16.5 n s$.

Fig. 5.7: Time-frequency analysis of the response data.

It required 200 poles to fit each parameter of the admittance matrix using conventional TD-VF. In comparison, the proposed algorithm required a total of 6 segments and 56 poles for each of the parameters. Sample accuracy comparisons of the admittance parameter fitting in the frequency-domain using both conventional TD-VF and the proposed algorithm are shown in Fig. 5.8. 


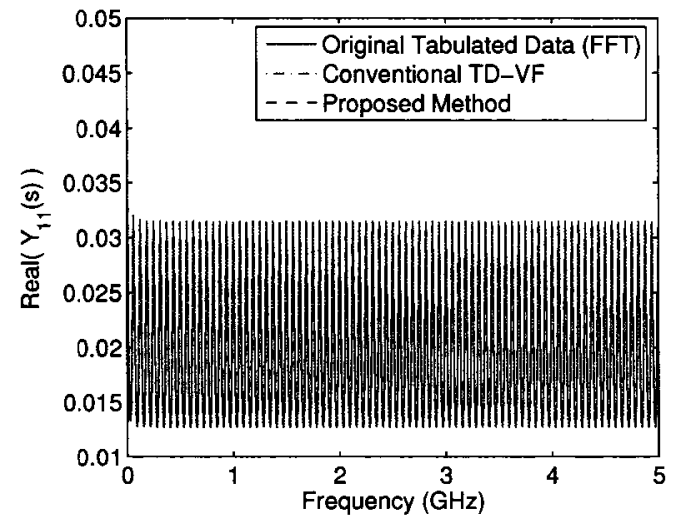

(a) Real part of $Y_{11}(s)$.

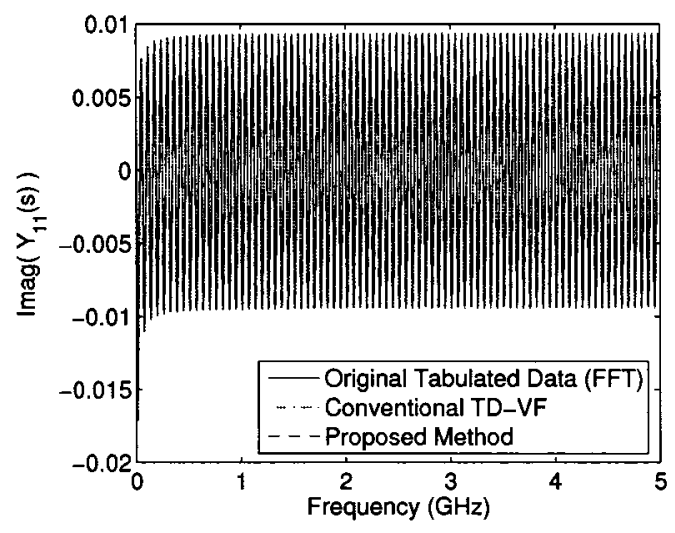

(c) Imaginary part of $Y_{11}(s)$.

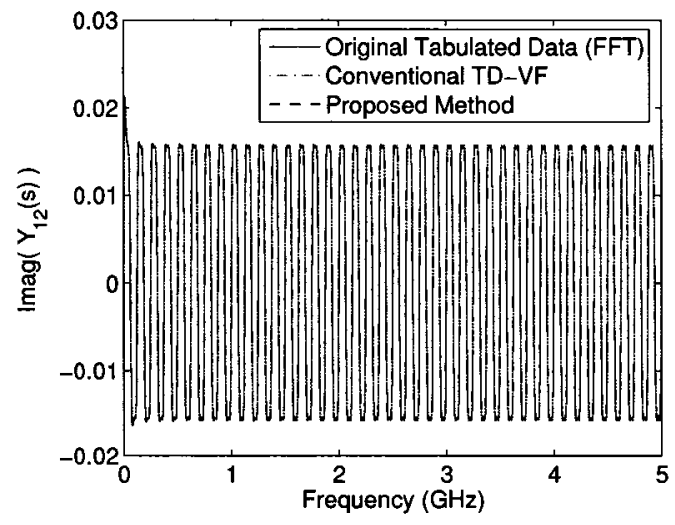

(e) Imaginary part of $Y_{12}(s)$.

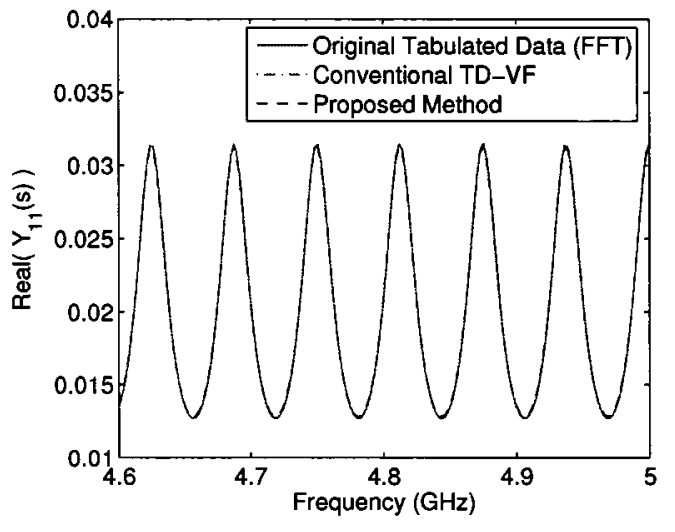

(b) An expanded view of Fig. 5.8(a).

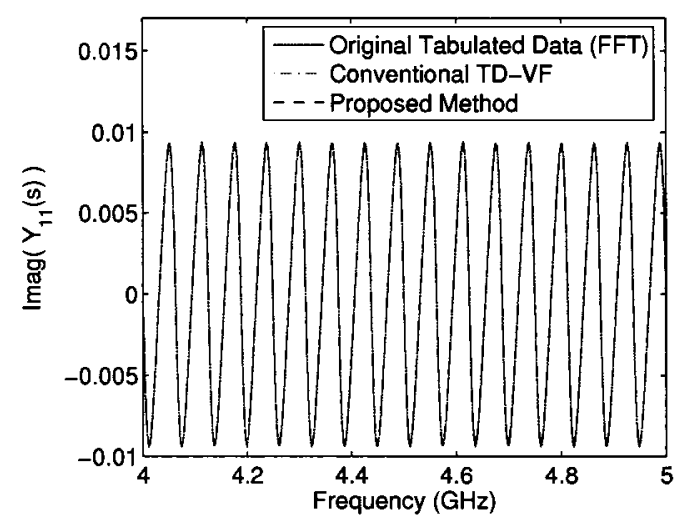

(d) An expanded view of Fig. 5.8(c).

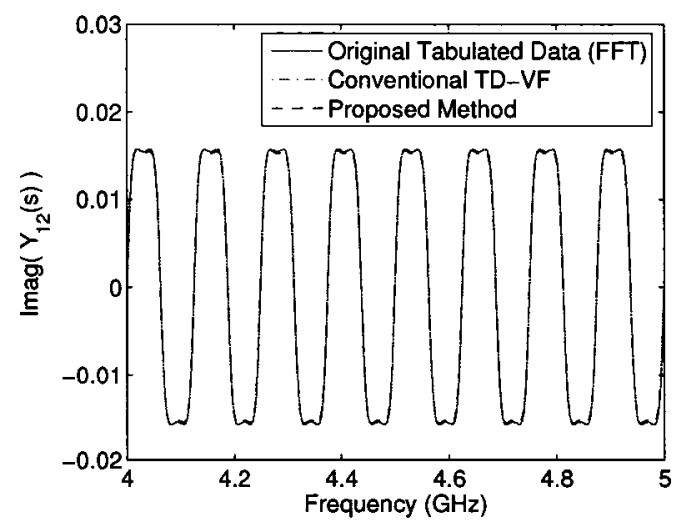

(f) An expanded view of Fig. 5.8(e).

Fig. 5.8: Frequency-domain comparisons for example 1. 


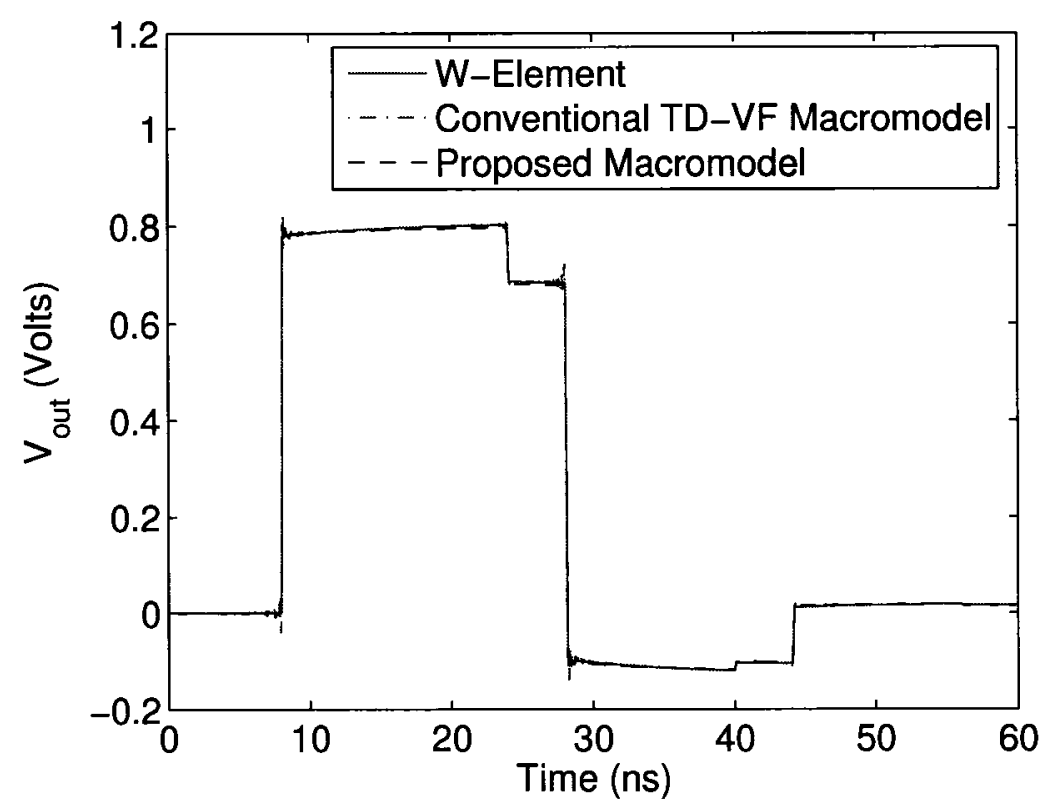

Fig. 5.9: Transient comparison for example 1 .

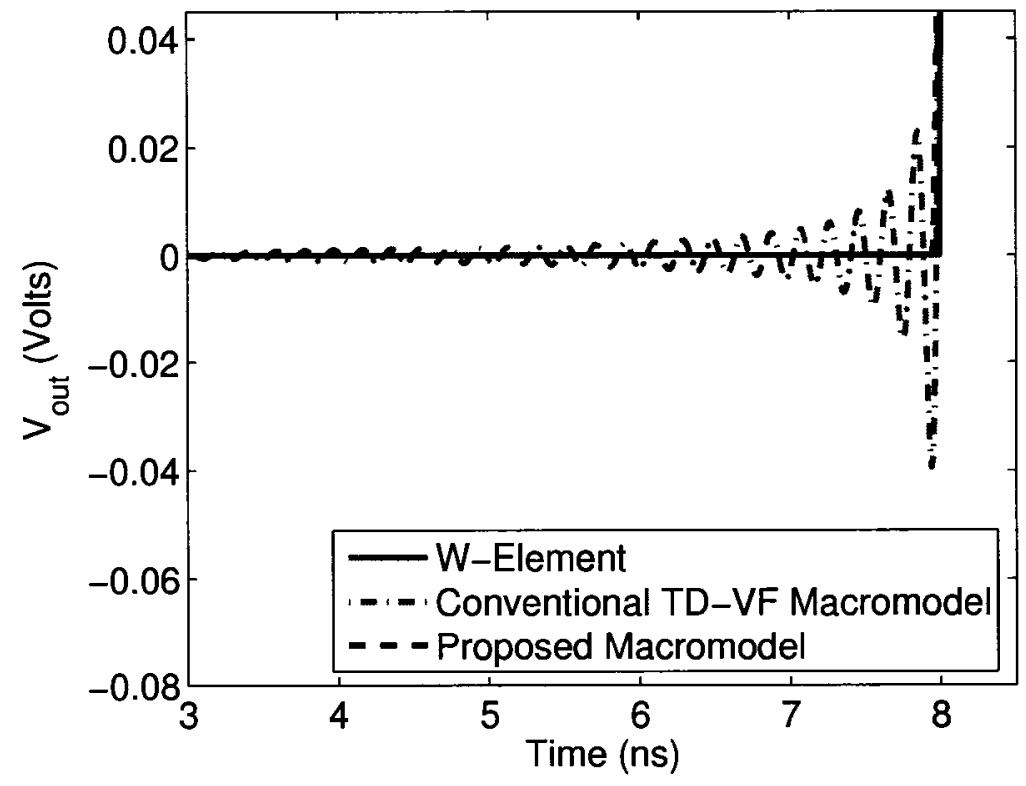

Fig. 5.10: Expanded view of the transient response in Fig. 5.9. 
Next, the macromodels from both conventional TD-VF and the proposed algorithm are simulated in HSPICE. The network was excited by a $20 \mathrm{~ns}$ wide pulse with $0.1 \mathrm{~ns}$ rise/fall times. It required 215.76 seconds to obtain the transient results using conventional TD-VF, compared to 4.03 seconds for the proposed macromodel. A comparison of the transient responses is shown in Figs. 5.9 and 5.10 against the benchmark response (W-element). As seen, the proposed macromodel is in good agreement with the benchmark response. In addition, by virtue of its delay based formulation, the proposed algorithm overcomes the ringing associated with the conventional TD-VF. This can be clearly seen in the expanded view provided in Fig. 5.10.

\subsubsection{Example 2}

In this example, a network (shown in Fig. 5.11) with multiple delays and multiple reflections is considered (the previous example contained a single delay with four reflections). Sample time-domain data is (along with its time-frequency analysis) shown in Fig. 5.12 for $y_{12}(t)$ of a two-port network. An expanded view of the timefrequency analysis is included for the purpose of clarity.

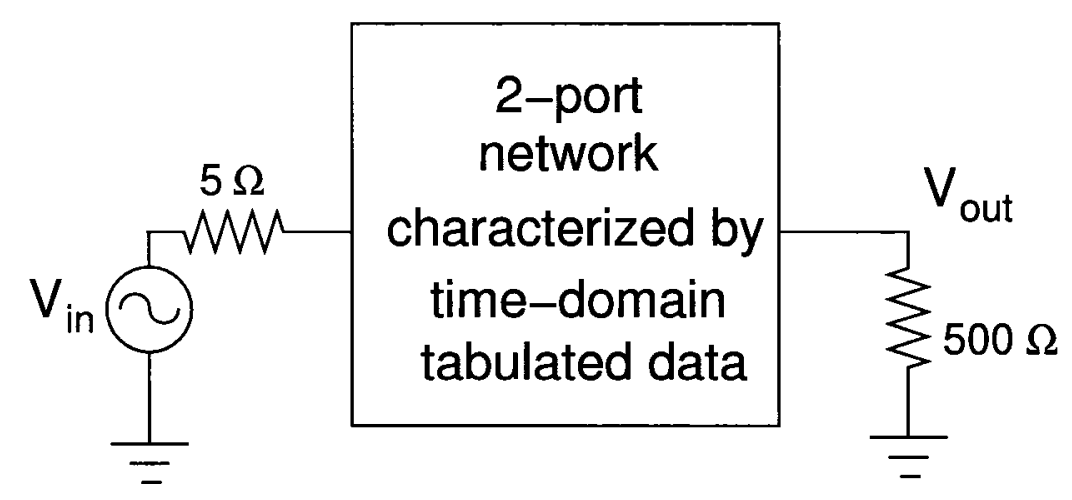

Fig. 5.11: Circuit for example 2 . 


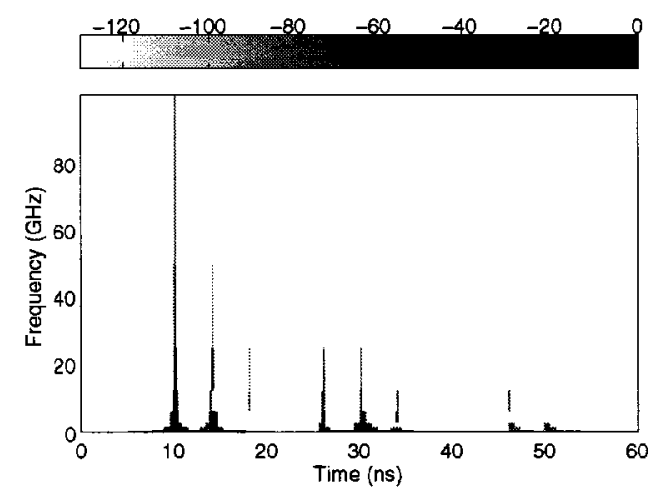

(a) Heisenberg tiles for wavelet decomposition.

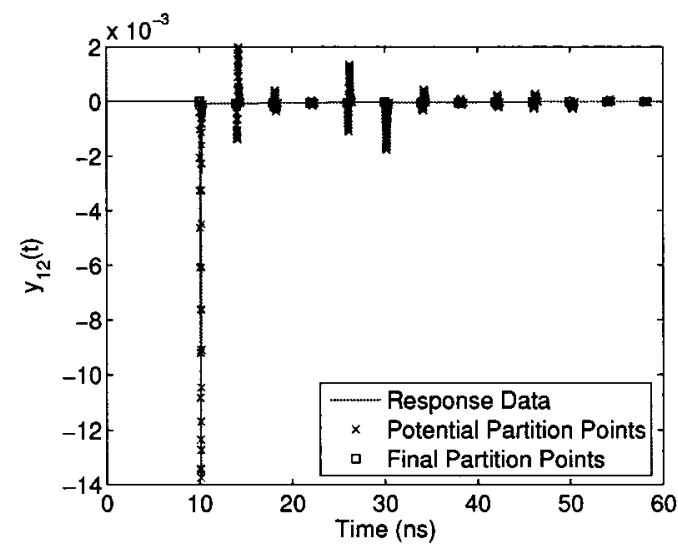

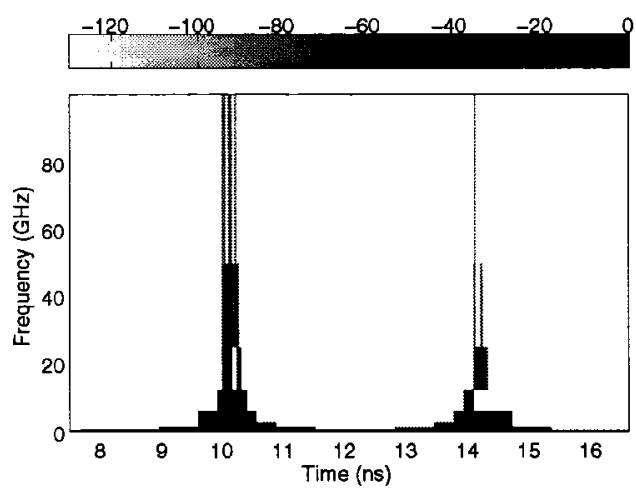

(b) An expanded view of Fig. 5.12(a) between $8 n s$ and $16 n s$.

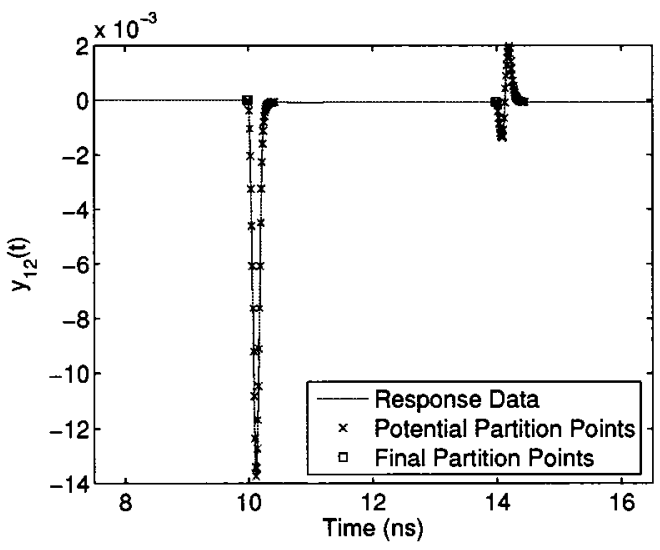

(c) Display of potential partition points and final (d) An expanded view of Fig. 5.12(c) between partition points. $8 n s$ and $16 n s$.

Fig. 5.12: Time-frequency analysis of the response data for example 2. 


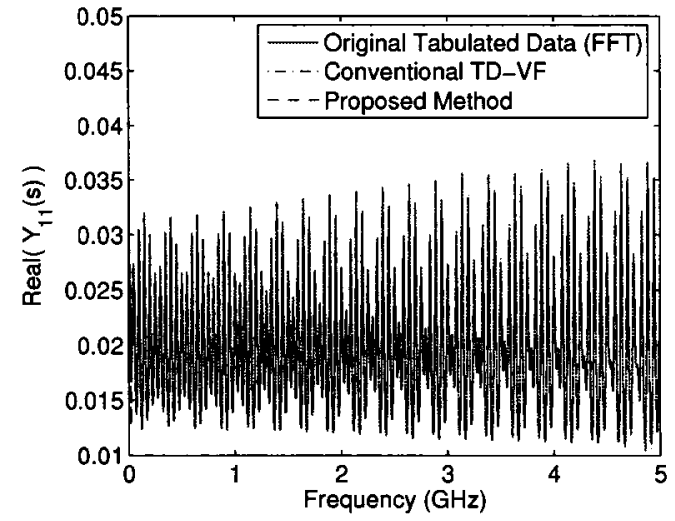

(a) Real part of $Y_{11}(s)$.

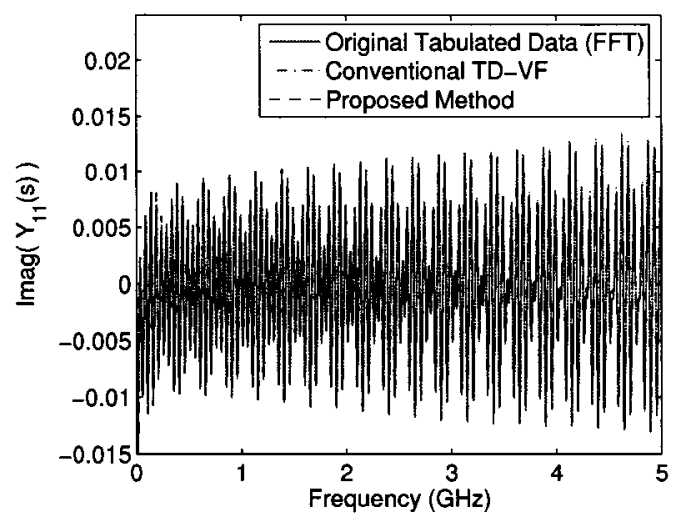

(c) Imaginary part of $Y_{11}(s)$.

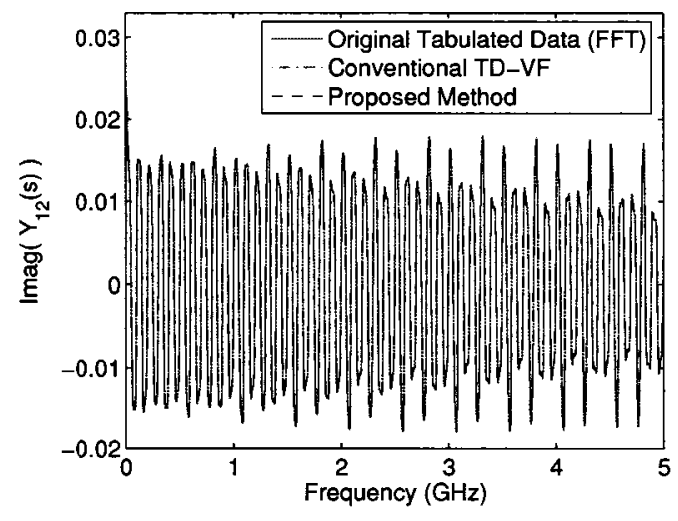

(e) Imaginary part of $Y_{12}(s)$.

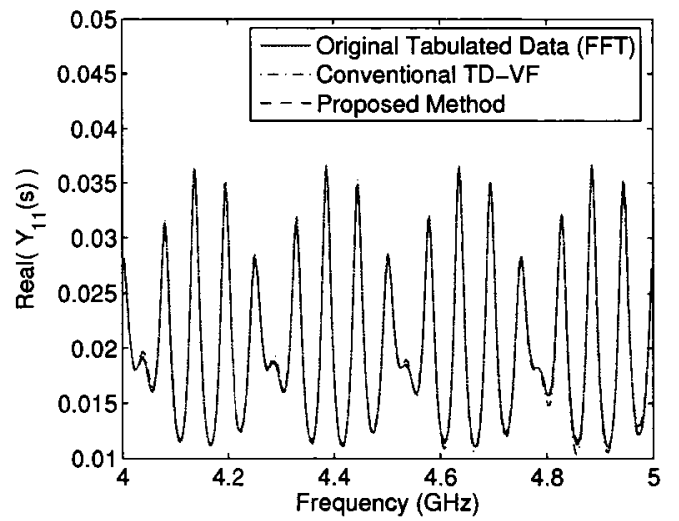

(b) An expanded view of Fig. 5.13(a).

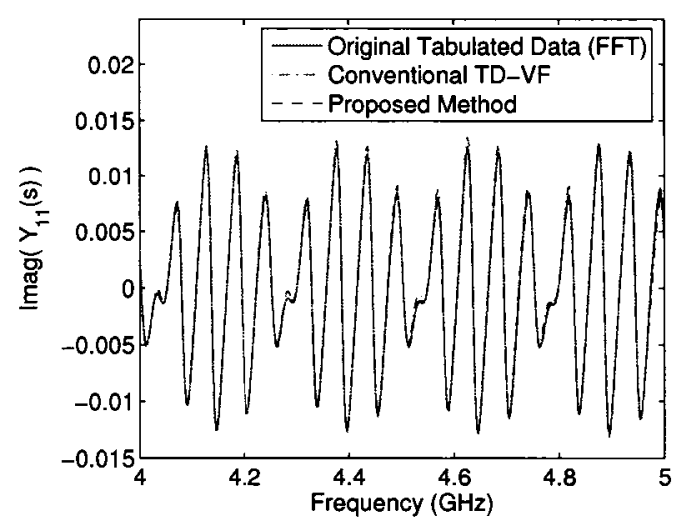

(d) An expanded view of Fig. 5.13(c).

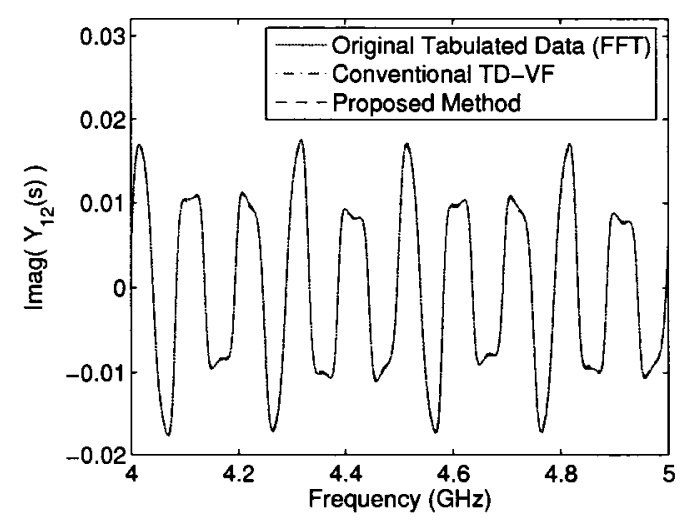

(f) An expanded view of Fig. 5.13(e).

Fig. 5.13: Frequency-domain comparisons for example 2. 


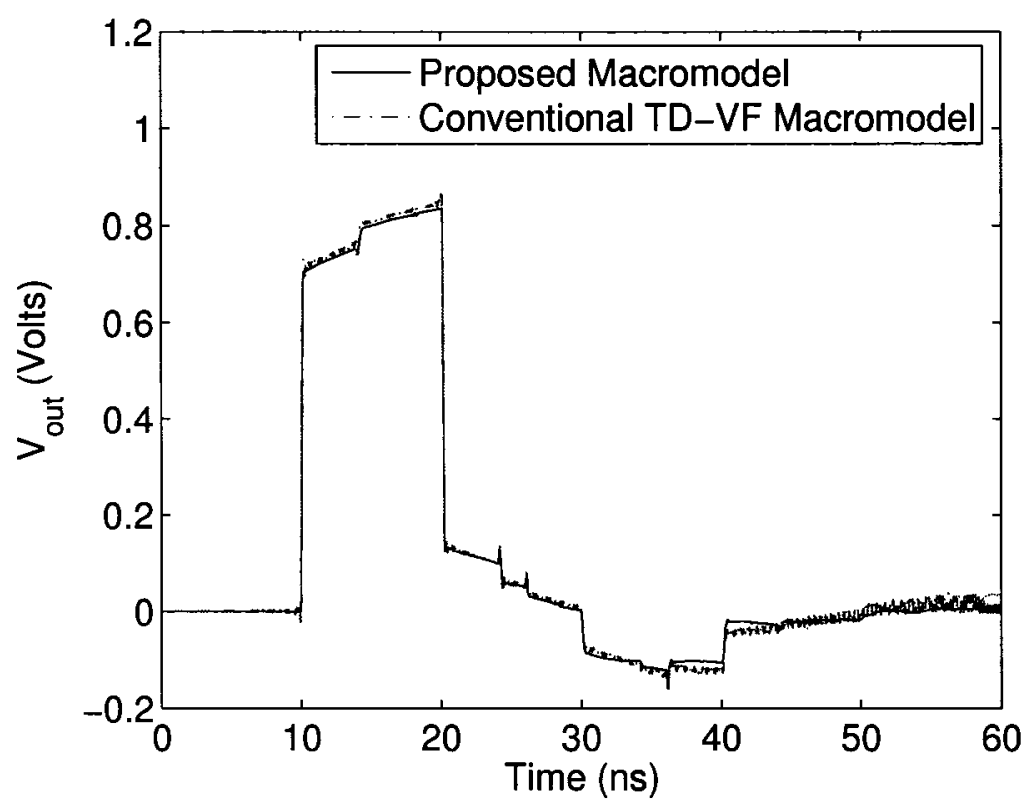

Fig. 5.14: Transient comparison for example 2.

The conventional TD-VF required 300 poles for each parameter, while the proposed algorithm required 10 segments and a total of 160 poles for each parameter. Sample comparisons of the admittance parameter fittings in the frequency-domain for both the conventional TD-VF algorithm and the proposed algorithm are shown in Fig. 5.13. It is clear that both fittings are in good agreement.

Next, the macromodel from both the conventional TD-VF algorithm and the proposed algorithm were simulated in HSPICE. Each network was excited by a pulse with $0.1 n s$ rise/fall times and a width of 10ns. The conventional TD-VF model required 356.81 seconds to simulate. In comparison, the proposed macromodel required 16.87 seconds. A comparison of the responses is shown in Fig. 5.14. In addition to giving significant speedup, the proposed algorithm overcomes the ringing that arises from conventional TD-VF macromodels, as can be seen from Fig. 5.14. 


\subsubsection{Example 3}

In this example, we consider the four-port network characterized by tabulated timedomain data shown in Fig. 5.15. The time-frequency analysis (for $\left.y_{11}(t)\right)$ is shown in Fig. 5.16. The conventional TD-VF model required a total of 2520 poles to fit the entire admittance response matrix. In comparison, the proposed algorithm required 84 segments and a total of 696 poles to fit the complete set of response data. A sample frequency-domain spectrum comparison (for $Y_{11}(s)$ ) is shown in Fig. 5.17. As seen, the conventional TD-VF and the proposed method are in good agreement.

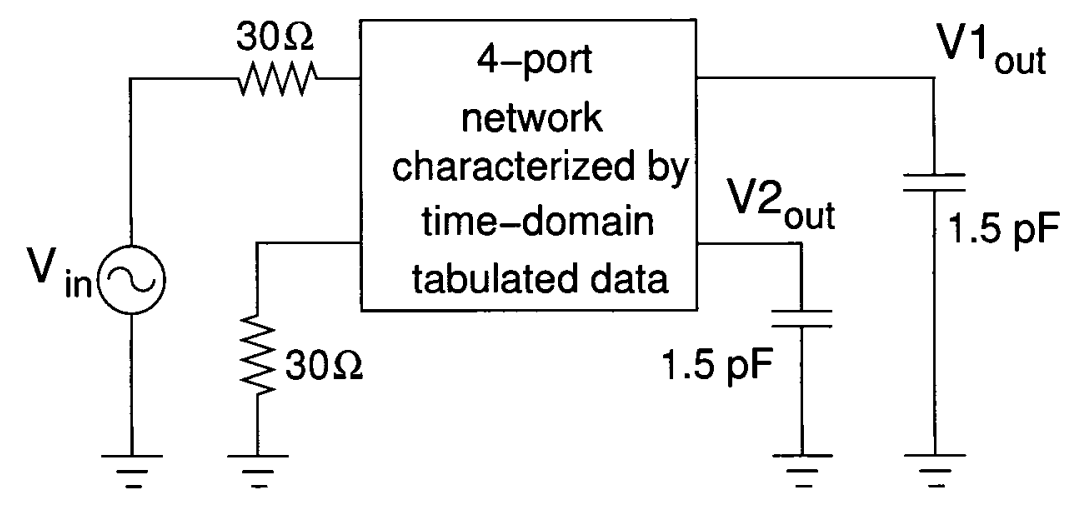

Fig. 5.15: Circuit for example 3.

Next, macromodels from both the conventional TD-VF and the proposed algorithm were simulated in HSPICE. Each macromodel was excited by a pulse with $0.1 n s$ rise/fall times and a pulse width of $20 n s$. The conventional TD-VF model required 107.98 seconds to simulate. In comparison, the proposed macromodel required 3.32 seconds. A comparison of the transient responses is shown in Fig. 5.18. Table 5.1 summarizes the CPU performance of the macromodels using the proposed method compared to the conventional TD-VF. 


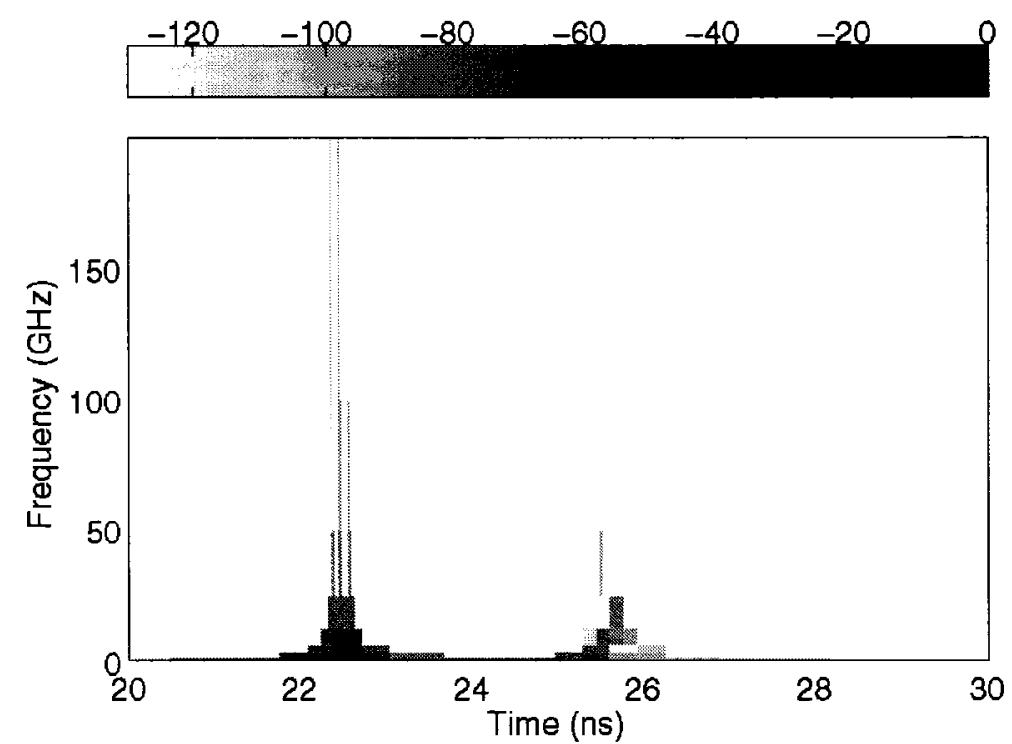

(a) Heisenberg tiles for wavelet decomposition.

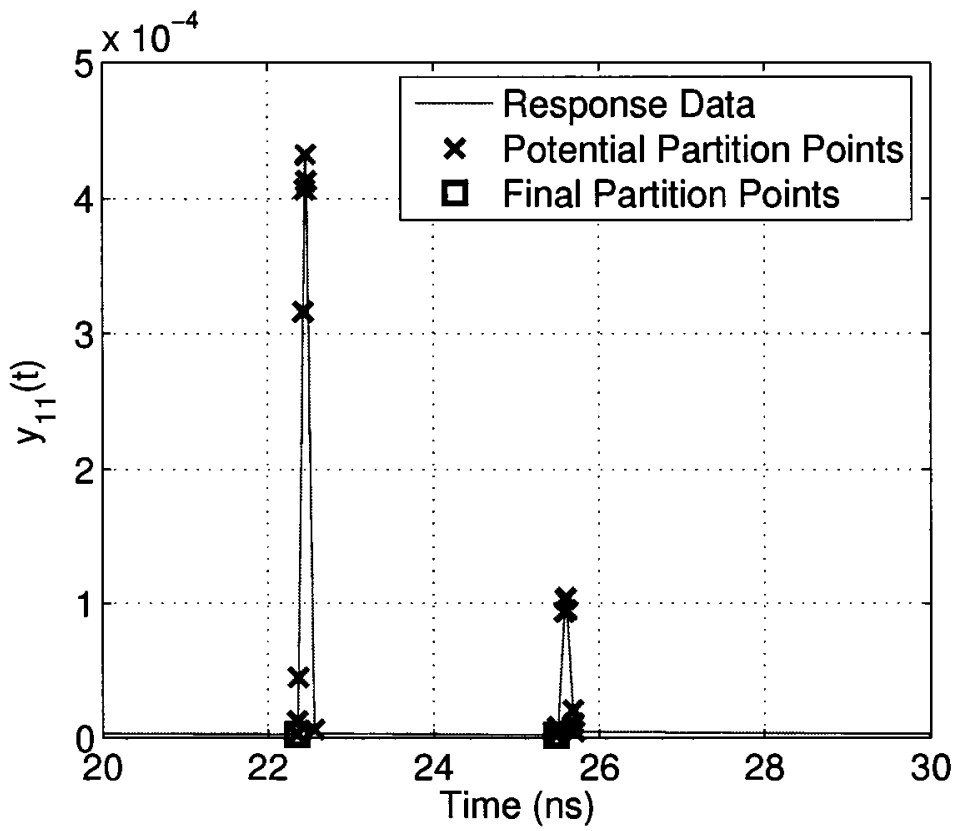

(b) Display of potential partition points and final partition points.

Fig. 5.16: Time-frequency analysis for example 3. 


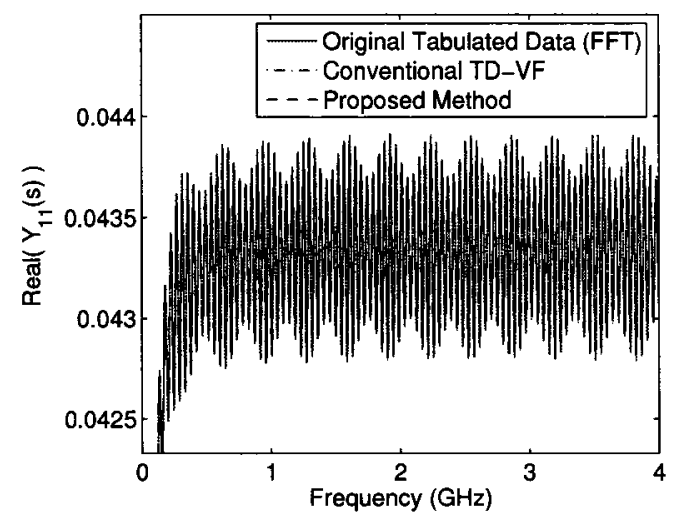

(a) Real part of $Y_{11}(s)$.

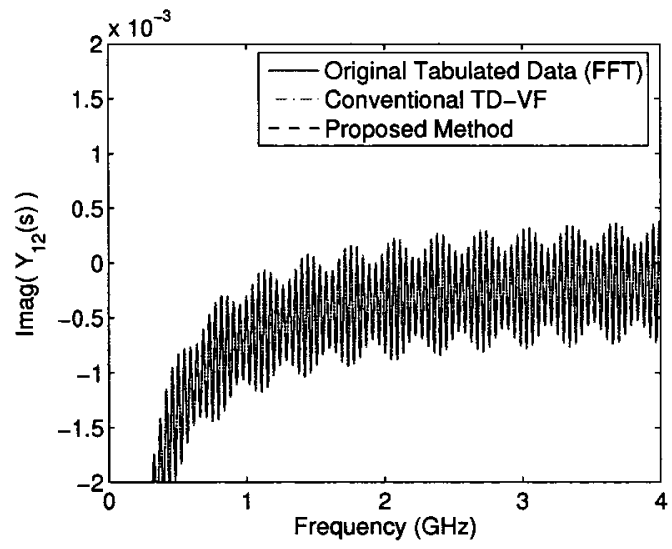

(c) Imaginary part of $Y_{12}(s)$.

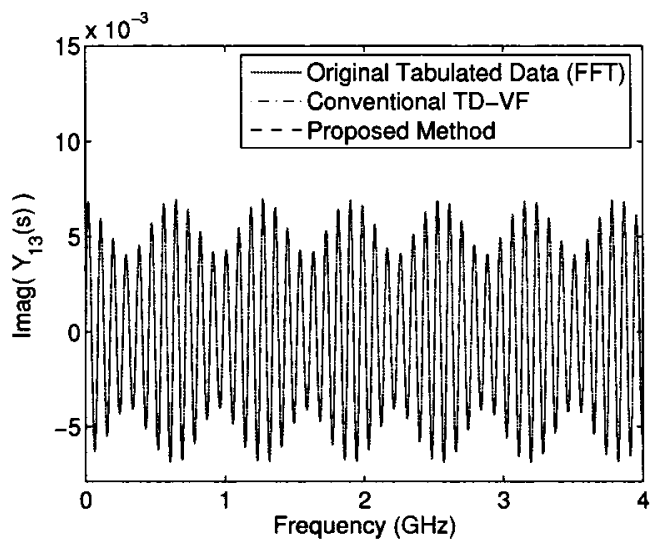

(e) Real part of $Y_{13}(s)$.

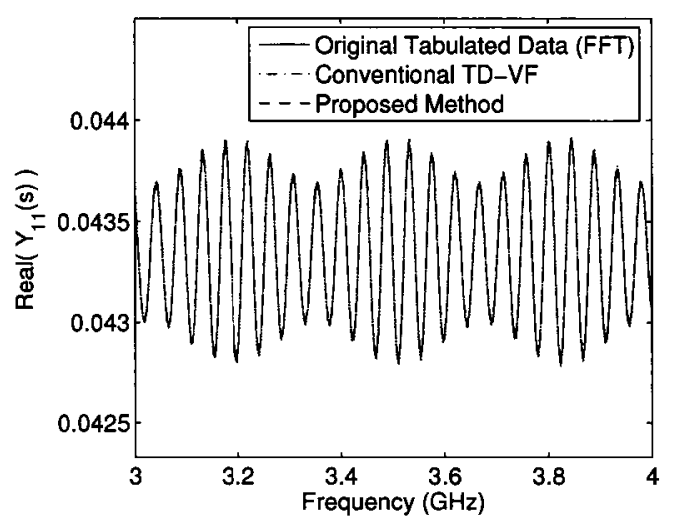

(b) An expanded view of Fig. 5.17(a).

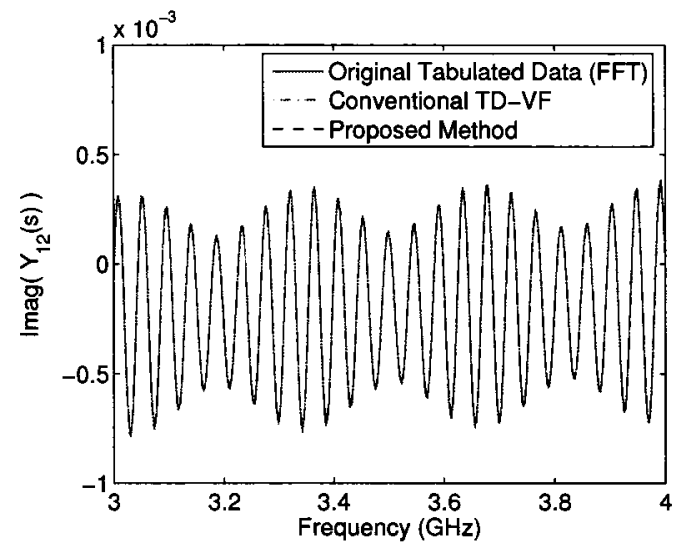

(d) An expanded view of Fig. 5.17(c).

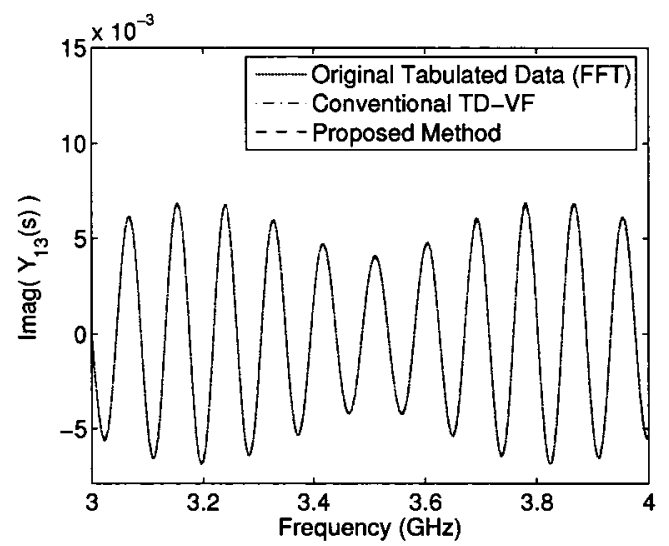

(f) An expanded view of Fig. 5.17(e).

Fig. 5.17: Frequency-domain comparisons for example 3. 


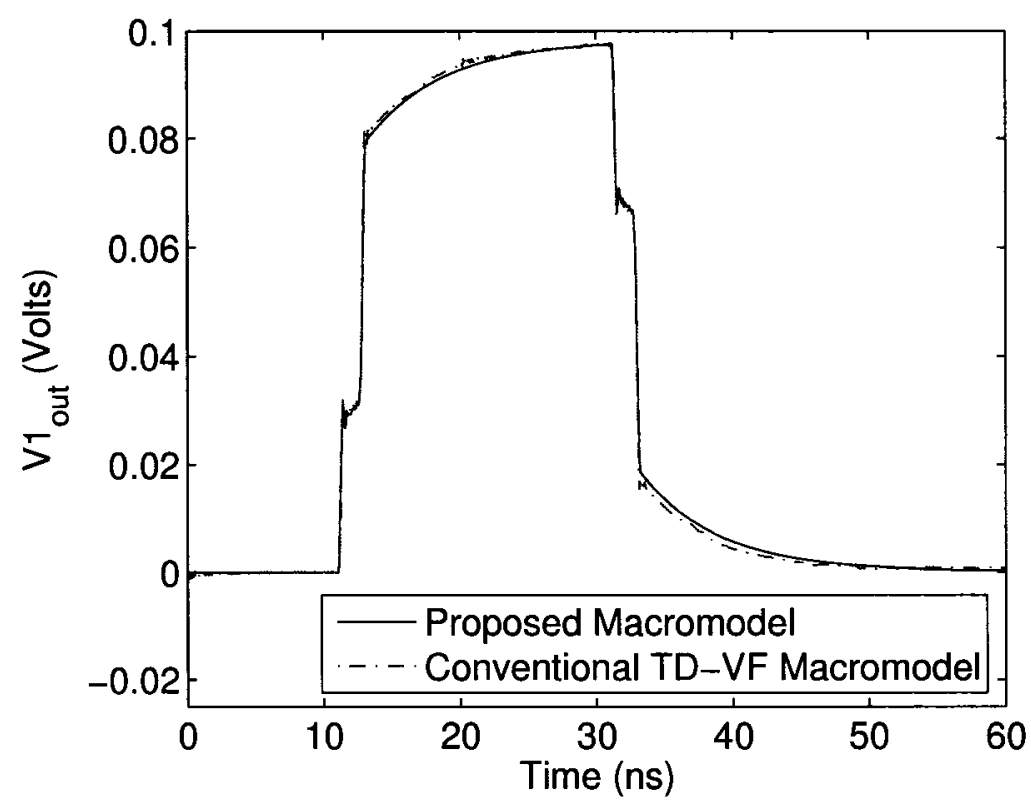

(a) Response of $V 1_{\text {out }}(t)$.

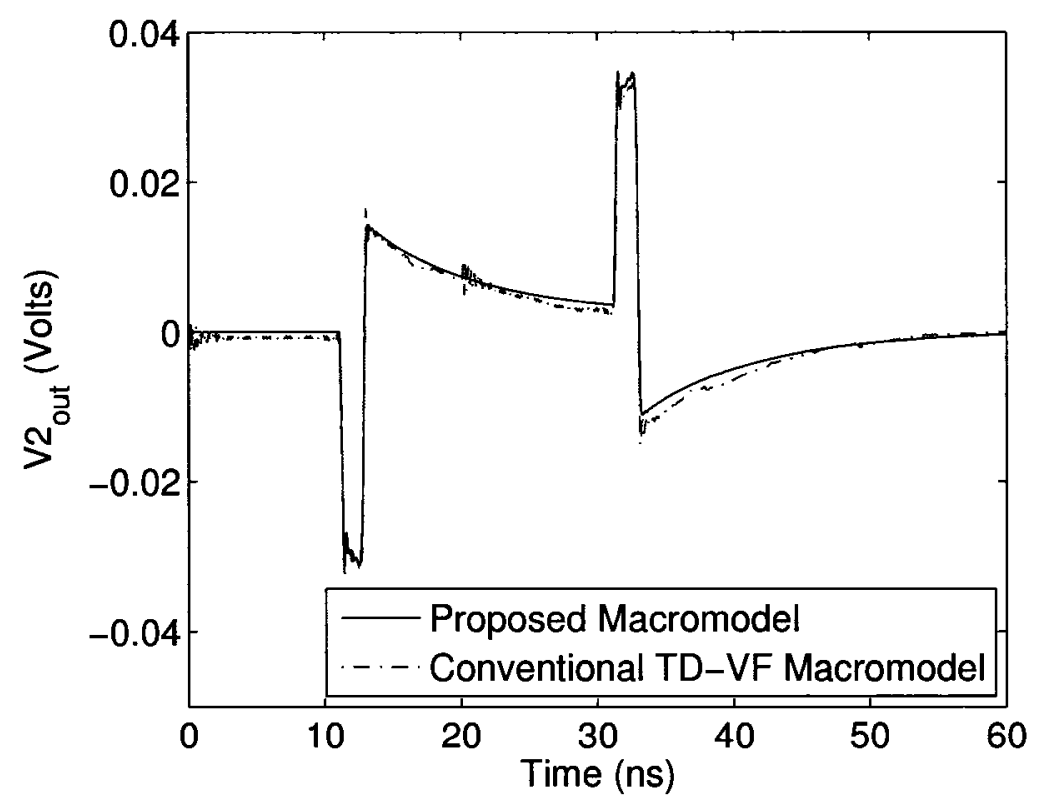

(b) Response of $V 2_{\text {out }}(t)$.

Fig. 5.18: Comparison of transient responses for example 3. 


\subsection{Summary}

In this chapter, a new method for generating low-order macromodels from tabulated time-domain data was developed. This algorithm can be applied to general systems, and is not limited to the matrix elements representing port-to-port delays in the system. In addition, this algorithm can extract more than just a single delay from a system, which is important for systems with multiple reflections. The proposed macromodel yields a significant speed-up compared to the conventional TD-VF algorithm, while overcoming the ringing in the response. 


\section{Chapter 6}

\section{Realization of Equivalent}

\section{Subcircuits from Delayed Rational}

\section{Functions}

\subsection{Introduction}

In chapters 4 and 5, delayed rational function approximation algorithms were introduced to model networks characterized by tabulated data. In order to simulate networks represented by delayed rational functions in SPICE, it is necessary to realize these equations as a set of equivalent subcircuits. In the case of rational functions, equivalent subcircuits are built using resistors, capacitors, and dependent sources [3], [42]. In this chapter, the standard macromodeling techniques for rational functions are expanded for delayed rational functions by introducing delay dependent sources. 
This chapter is organized as follows. Section 6.2 derives the delayed state-space formulation for systems described by delayed rational functions. Next, section 6.3 modifies the state-space formulation for delayed rational functions with complex poles. Finally, section 6.4 describes how to construct a set of equivalent subcircuits from a delayed state-space formulation.

\subsection{Macromodel Synthesis}

Consider the linear subnetwork $\pi$ in Fig. 6.1. The Y parameters are of the form

$$
\left[\begin{array}{cccc}
Y_{11} & Y_{12} & \ldots & Y_{1 n_{\pi}} \\
\vdots & \vdots & \ddots & \vdots \\
Y_{n_{\pi} 1} & Y_{n_{\pi} 2} & \ldots & Y_{n_{\pi} n_{\pi}}
\end{array}\right]\left[\begin{array}{c}
V_{1} \\
\vdots \\
V_{n_{\pi}}
\end{array}\right]=\left[\begin{array}{c}
I_{1} \\
\vdots \\
I_{n_{\pi}}
\end{array}\right]
$$

where $n_{\pi}$ is the number of ports, $\left\{V_{i}\right\}$ are the port input voltages, and $\left\{I_{i}\right\}$ are the port output currents. The algorithms from chapter 4 and 5 fit each of the elements, $Y_{i j}(s)$, with a delayed rational function of the form

$$
Y_{i j}(s)=\sum_{a=0}^{Q^{i, j}-1}\left(d_{a}^{i, j}+\sum_{m=1}^{M_{a}^{i, j}} \frac{k_{m, a}^{i, j}}{s-p_{m, a}^{i, j}}\right) e^{-s \tau_{a}}
$$

where $Q^{i, j}$ is the number of delays in each element. This macromodel results in a delayed state-space formulation

$$
\begin{aligned}
& \dot{\boldsymbol{x}}(t)=\mathbf{A} \boldsymbol{x}(t)+\mathbf{B} \boldsymbol{\phi}(t) \\
& \boldsymbol{y}(t)=\sum_{q=0}^{Q-1} \mathbf{C}_{q} \boldsymbol{x}\left(t-\tau_{q}\right) u\left(t-\tau_{q}\right)+\sum_{q=0}^{Q-1} \mathbf{D}_{q} \boldsymbol{\phi}\left(t-\tau_{q}\right) u\left(t-\tau_{q}\right),
\end{aligned}
$$


where $Q$ is the total number of delays in the system, $\tau_{q}$ is the $q^{\text {th }}$ delay, $\mathbf{C}_{q} \in \mathbb{R}^{n_{\pi \times n}}$ relates the delayed state-variables to the outputs $(\boldsymbol{y}), \mathbf{D}_{q} \in \mathbb{R}^{n_{\pi} \times n_{\pi}}$ relates the delayed input directly to the output, $\boldsymbol{x}\left(t-\tau_{q}\right) u\left(t-\tau_{q}\right)$ is the delayed state vector of length $n$, and $\phi\left(t-\tau_{q}\right) u\left(t-\tau_{q}\right)$ is the delayed input vector of length $n_{\pi} . \mathbf{A} \in \mathbb{R}^{n \times n}$ is the state matrix and $\mathbf{B} \in \mathbb{R}^{n \times n_{\pi}}$ relates the inputs to the state variables.

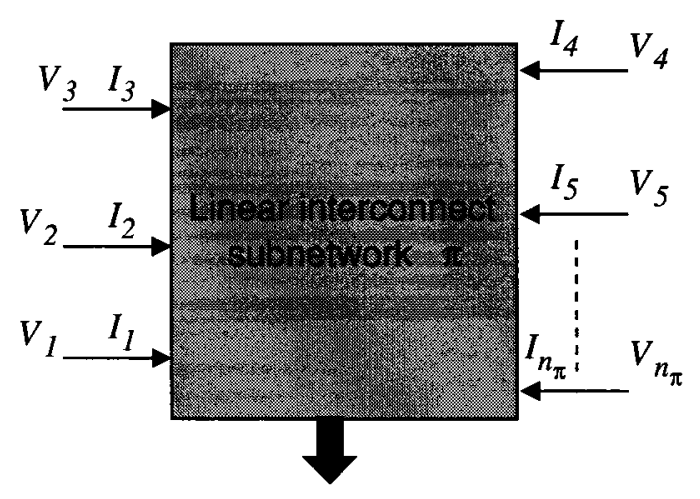

Fig. 6.1: Multiport linear subnetwork $\pi$.

\subsubsection{Example: Macromodel Synthesis}

In order to illustrate the conversion of a matrix with elements defined by delayed rational functions to a delayed state-space system, consider a two-port example with two common poles and two common delays, $\tau_{0}=0$ and $\tau_{1}=\tau$. The transfer function is described by

$$
\left[\begin{array}{ll}
\sum_{a=0}^{1}\left(d_{a}^{1,1}+\sum_{m=1}^{2} \frac{k_{m, a}^{1,1}}{s-p_{m}}\right) e^{-s \tau_{a}} & \sum_{a=0}^{1}\left(d_{a}^{1,2}+\sum_{m=1}^{2} \frac{k_{m, a}^{1,2}}{s-p_{m}}\right) e^{-s \tau_{a}} \\
\sum_{a=0}^{1}\left(d_{a}^{2,1}+\sum_{m=1}^{2} \frac{k_{m, a}^{2,1}}{s-p_{m}}\right) e^{-s \tau_{a}} & \sum_{a=0}^{1}\left(d_{a}^{2,2}+\sum_{m=1}^{2} \frac{k_{m, a}^{2,2}}{s-p_{m}}\right) e^{-s \tau_{a}}
\end{array}\right]\left[\begin{array}{c}
V_{1} \\
V_{2}
\end{array}\right]=\left[\begin{array}{c}
I_{1} \\
I_{2}
\end{array}\right] .
$$


Let $X_{1}, X_{2}, X_{3}$, and $X_{4}$ be the state variables in the frequency-domain, and let $\left\{x_{i}\right\}$, $\left\{v_{i}\right\}$ be the time-domain equivalent of $\left\{X_{i}\right\},\left\{V_{i}\right\}$, respectively, such that

$$
\begin{array}{lll}
\left(s-p_{1}\right) X_{1}=V_{1} & \Rightarrow & \dot{x}_{1}(t)=p_{1} x_{1}(t)+v_{1}(t), \\
\left(s-p_{2}\right) X_{2}=V_{1} & \Rightarrow & \dot{x}_{2}(t)=p_{2} x_{2}(t)+v_{1}(t), \\
\left(s-p_{1}\right) X_{3}=V_{2} & \Rightarrow & \dot{x}_{3}(t)=p_{1} x_{3}(t)+v_{2}(t), \\
\left(s-p_{2}\right) X_{4}=V_{2} & \Rightarrow & \dot{x}_{4}(t)=p_{2} x_{4}(t)+v_{2}(t) .
\end{array}
$$

Substituting (6.5) - (6.8) into (6.4) and taking the inverse Laplace transform yields

$$
\begin{aligned}
& {\left[\begin{array}{c}
i_{1}(t) \\
i_{2}(t)
\end{array}\right]=\left[\begin{array}{llll}
k_{1,0}^{1,1} & k_{2,0}^{1,1} & k_{1,0}^{1,2} & k_{2,0}^{1,2} \\
k_{1,0}^{2,1} & k_{2,0}^{2,1} & k_{1,0}^{2,2} & k_{2,0}^{2,2}
\end{array}\right]\left[\begin{array}{c}
x_{1}(t) \\
x_{2}(t) \\
x_{3}(t) \\
x_{4}(t)
\end{array}\right]+\left[\begin{array}{cc}
d_{0}^{1,1} & d_{0}^{1,2} \\
d_{0}^{2,1} & d_{0}^{2,2}
\end{array}\right]\left[\begin{array}{l}
v_{1}(t) \\
v_{2}(t)
\end{array}\right]} \\
& +\left[\begin{array}{llll}
k_{1,1}^{1,1} & k_{2,1}^{1,1} & k_{1,1}^{1,2} & k_{2,1}^{1,2} \\
k_{1,1}^{2,1} & k_{2,1}^{2,1} & k_{1,1}^{2,2} & k_{2,1}^{2,2}
\end{array}\right]\left[\begin{array}{l}
x_{1}(t-\tau) \\
x_{2}(t-\tau) \\
x_{3}(t-\tau) \\
x_{4}(t-\tau)
\end{array}\right] u(t-\tau) \\
& +\left[\begin{array}{cc}
d_{1}^{1,1} & d_{1}^{1,2} \\
d_{1}^{2,1} & d_{1}^{2,2}
\end{array}\right]\left[\begin{array}{c}
v_{1}(t-\tau) \\
v_{2}(t-\tau)
\end{array}\right] u(t-\tau)
\end{aligned}
$$


where $i_{1}$ and $i_{2}$ are the output currents in the time-domain. Next, note that (6.5) (6.8) can be written in matrix form as

$$
\left[\begin{array}{c}
\dot{x}_{1}(t) \\
\dot{x}_{2}(t) \\
\dot{x}_{3}(t) \\
\dot{x}_{4}(t)
\end{array}\right]=\left[\begin{array}{cccc}
p_{1} & 0 & 0 & 0 \\
0 & p_{2} & 0 & 0 \\
0 & 0 & p_{1} & 0 \\
0 & 0 & 0 & p_{2}
\end{array}\right]\left[\begin{array}{c}
x_{1}(t) \\
x_{2}(t) \\
x_{3}(t) \\
x_{4}(t)
\end{array}\right]+\left[\begin{array}{cc}
1 & 0 \\
1 & 0 \\
0 & 1 \\
0 & 1
\end{array}\right]\left[\begin{array}{l}
v_{1}(t) \\
v_{2}(t)
\end{array}\right] .
$$

Finally, (6.9) and (6.10) can be re-written as

$$
\begin{aligned}
& \dot{\boldsymbol{x}}(t)=\mathbf{A} \boldsymbol{x}(t)+\mathbf{B} \boldsymbol{u}(t) \\
& \boldsymbol{y}(t)=\mathbf{C}_{0} \boldsymbol{x}(t)+\mathbf{C}_{1} \boldsymbol{x}(t-\tau) u(t-\tau)+\mathbf{D}_{0} \phi(t)+\mathbf{D}_{1} \phi(t-\tau) u(t-\tau)
\end{aligned}
$$

which is a delayed state-space formulation.

\subsection{Inclusion of Complex Poles}

In most cases, the matrix-transfer function, $\mathbf{Y}(s)$, in (6.1) will contain complex poles. Then, the conversion needs to be treated differently since complex values do not have a direct meaning in the time-domain. All the residues and poles obtained from fitting the data are complex conjugates by construction, and therefore its roots are all complex conjugates as well [30], [36]. The delayed state-space realization for complex pole-pairs uses techniques similar to the Jordan-form state-space realization described 
in [3]. In this case, the formulation becomes

$$
\begin{aligned}
{\left[\begin{array}{c}
\dot{\boldsymbol{x}}_{\mathbf{1}}(t) \\
\dot{\boldsymbol{x}}_{\mathbf{2}}(t)
\end{array}\right] } & =\left[\begin{array}{cc}
\mathbf{A} & \mathbf{0} \\
\mathbf{0} & \mathbf{A}^{*}
\end{array}\right]\left[\begin{array}{l}
\boldsymbol{x}_{\mathbf{1}}(t) \\
\boldsymbol{x}_{\mathbf{2}}(t)
\end{array}\right]+\left[\begin{array}{l}
\boldsymbol{b} \\
\mathbf{0}
\end{array}\right] \boldsymbol{\phi}(t) \\
\boldsymbol{y}(t) & =\sum_{a=0}^{Q-1}\left[\begin{array}{ll}
\mathbf{C}_{\mathbf{a}} & \mathbf{C}_{\mathbf{a}}^{*}
\end{array}\right]\left[\begin{array}{l}
\boldsymbol{x}_{1}\left(t-\tau_{a}\right) \\
\boldsymbol{x}_{2}\left(t-\tau_{a}\right)
\end{array}\right] u\left(t-\tau_{a}\right),
\end{aligned}
$$

where $\mathbf{A}$ is a diagonal matrix of the poles of $\mathbf{Y}_{\pi}(s), \mathbf{A}^{*}$ is the complex conjugate of A. Next, an equivalence transformation is applied to the system such that

$$
\hat{\boldsymbol{x}}\left(t-\tau_{a}\right) u\left(t-\tau_{a}\right)=\mathbf{T} \boldsymbol{x}\left(t-\tau_{a}\right) u\left(t-\tau_{a}\right), \quad \mathbf{T}=\left[\begin{array}{cc}
\mathbf{I} & \mathbf{I} \\
j \mathbf{I} & -j \mathbf{I}
\end{array}\right]
$$

This transformation can be used with (6.13) to obtain

$$
\begin{aligned}
& {\left[\begin{array}{c}
\dot{\hat{\boldsymbol{x}}}_{1}(t) \\
\dot{\hat{\boldsymbol{x}}}_{2}(t)
\end{array}\right]=\left[\begin{array}{cc}
\mathfrak{R e}(\mathbf{A}) & \mathfrak{I m}(\mathbf{A}) \\
-\mathfrak{I m}(\mathbf{A}) & \mathfrak{R e}(\mathbf{A})
\end{array}\right]\left[\begin{array}{c}
\hat{\boldsymbol{x}}_{1}(t) \\
\hat{\boldsymbol{x}}_{2}(t)
\end{array}\right]+\left[\begin{array}{c}
2 \mathfrak{R e}(\boldsymbol{b}) \\
\mathbf{0}
\end{array}\right] \boldsymbol{\phi}(t)}
\end{aligned}
$$

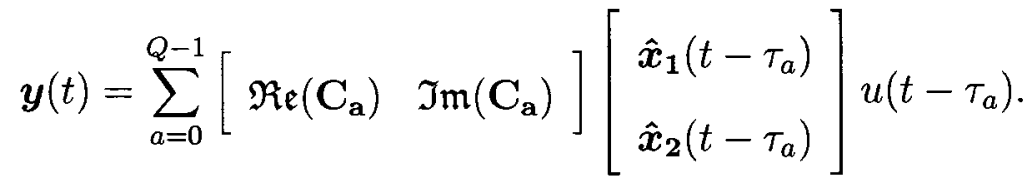

\subsubsection{Example: Complex Pole Modifications}

In order to illustrate the transformations to the delayed state-space system for complex poles, consider a two-port network containing a single complex pole-pair and delays $\tau_{1}=0$ and $\tau_{2}=\tau$. Let $p_{1,2}=w \pm j z$ and $k_{i, j, a}=(r \pm j v)_{a}^{i, j}$ be the corre-

sponding residues at different ports and corresponding to the $a^{\text {th }}$ delay. Then, the 
realization of $(6.13 \mathrm{a})$ for this example is

$$
\left[\begin{array}{c}
\dot{x}_{1}(t) \\
\dot{x}_{2}(t) \\
\dot{x}_{3}(t) \\
\dot{x}_{4}(t)
\end{array}\right]=\left[\begin{array}{cccc}
w+i z & 0 & 0 & 0 \\
0 & w+i z & 0 & 0 \\
0 & 0 & w-i z & 0 \\
0 & 0 & 0 & w-i z
\end{array}\right]\left[\begin{array}{c}
x_{1}(t) \\
x_{2}(t) \\
x_{3}(t) \\
x_{4}(t)
\end{array}\right]+\left[\begin{array}{ll}
1 & 0 \\
0 & 1 \\
1 & 0 \\
0 & 1
\end{array}\right]\left[\begin{array}{l}
v_{1}(t) \\
v_{2}(t)
\end{array}\right],
$$

while the realization of $(6.13 \mathrm{~b})$ is

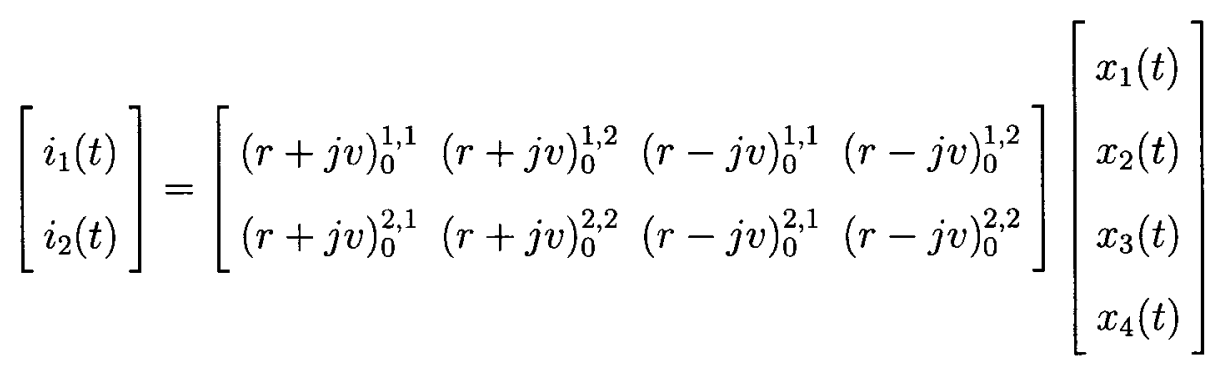

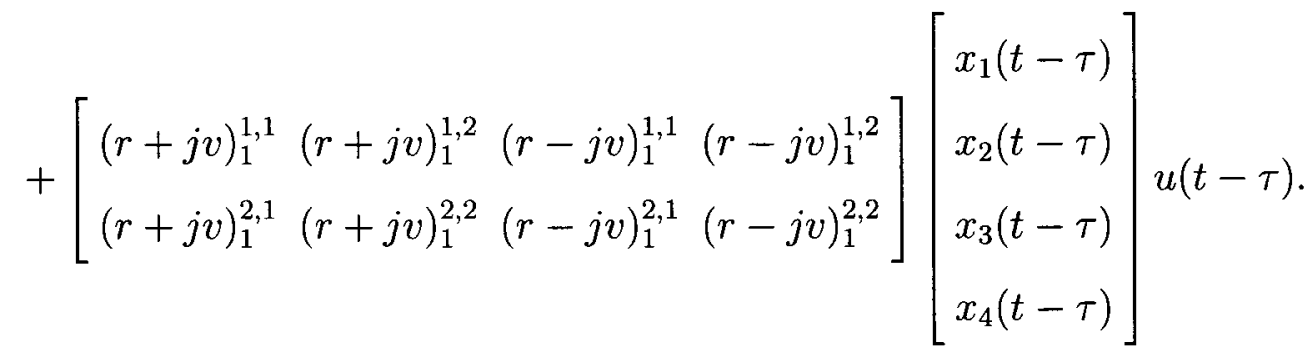

Define the transformation matrix

$$
\mathbf{T}=\left[\begin{array}{cccc}
1 & 0 & 1 & 0 \\
0 & 1 & 0 & 1 \\
j & 0 & -j & 0 \\
0 & j & 0 & -j
\end{array}\right]
$$


This transformation matrix can be used to obtain the realization for this two-port system in the form of $(6.15)$

$$
\begin{aligned}
& {\left[\begin{array}{c}
\dot{\hat{x}}_{1}(t) \\
\dot{\hat{x}}_{2}(t) \\
\dot{\hat{x}}_{3}(t) \\
\dot{\hat{x}}_{4}(t)
\end{array}\right]=\left[\begin{array}{cccc}
w & 0 & z & 0 \\
0 & w & 0 & z \\
-z & 0 & w & 0 \\
0 & -z & 0 & w
\end{array}\right]\left[\begin{array}{c}
\hat{x}_{1}(t) \\
\hat{x}_{2}(t) \\
\hat{x}_{3}(t) \\
\hat{x}_{4}(t)
\end{array}\right]+\left[\begin{array}{ll}
2 & 0 \\
0 & 2 \\
0 & 0 \\
0 & 0
\end{array}\right]\left[\begin{array}{c}
v_{1}(t) \\
v_{2}(t)
\end{array}\right]} \\
& {\left[\begin{array}{c}
i_{1}(t) \\
i_{2}(t)
\end{array}\right]=\left[\begin{array}{cccc}
r_{0}^{1,1} & r_{0}^{1,2} & v_{0}^{1,1} & v_{0}^{1,2} \\
r_{0}^{2,1} & r_{0}^{2,2} & v_{0}^{2,1} & v_{0}^{2,2}
\end{array}\right]\left[\begin{array}{c}
\hat{x}_{1}(t) \\
\hat{x}_{2}(t) \\
\hat{x}_{3}(t) \\
\hat{x}_{4}(t)
\end{array}\right]} \\
& +\left[\begin{array}{cccc}
r_{1}^{1,1} & r_{1}^{1,2} & v_{1}^{1,1} & v_{1}^{1,2} \\
r_{1}^{2,1} & r_{1}^{2,2} & v_{1}^{2,1} & v_{1}^{2,2}
\end{array}\right]\left[\begin{array}{c}
\hat{x}_{1}(t-\tau) \\
\hat{x}_{2}(t-\tau) \\
\hat{x}_{3}(t-\tau) \\
\hat{x}_{4}(t-\tau)
\end{array}\right] u(t-\tau)
\end{aligned}
$$

\subsection{Construction of Equivalent Subcircuits}

Once the delayed rational functions are transformed into the delayed state-space form of (6.3), they can be converted into a set of equivalent subcircuits. The conversion of DDEs to equivalent subcircuits can be done in several ways, one of these methods is presented in this section. Consider a two-port network in the form of (6.3) with two 
states and two delays at $\tau_{0}=0$ and $\tau_{1}=\tau$. This system can be written

$$
\begin{aligned}
{\left[\begin{array}{c}
\dot{x}_{1}(t) \\
\dot{x}_{2}(t)
\end{array}\right]=} & {\left[\begin{array}{cc}
a_{11} & a_{12} \\
a_{21} & a_{22}
\end{array}\right]\left[\begin{array}{l}
x_{1}(t) \\
x_{2}(t)
\end{array}\right]+\left[\begin{array}{ll}
b_{11} & b_{12} \\
b_{21} & b_{22}
\end{array}\right]\left[\begin{array}{l}
v_{1}(t) \\
v_{2}(t)
\end{array}\right] } \\
{\left[\begin{array}{c}
i_{1}(t) \\
i_{2}(t)
\end{array}\right]=} & {\left[\begin{array}{ll}
c_{11,0} & c_{12,0} \\
c_{21,0} & c_{22,0}
\end{array}\right]\left[\begin{array}{l}
x_{1}(t) \\
x_{2}(t)
\end{array}\right]+\left[\begin{array}{ll}
c_{11,1} & c_{12,1} \\
c_{21,1} & c_{22,1}
\end{array}\right]\left[\begin{array}{l}
x_{1}(t-\tau) \\
x_{2}(t-\tau)
\end{array}\right] u(t-\tau) } \\
& +\left[\begin{array}{cc}
d_{11,0} & d_{12,0} \\
d_{21,0} & d_{22,0}
\end{array}\right]\left[\begin{array}{l}
v_{1}(t) \\
v_{2}(t)
\end{array}\right]+\left[\begin{array}{ll}
d_{11,1} & d_{12,1} \\
d_{21,1} & d_{22,1}
\end{array}\right]\left[\begin{array}{l}
v_{1}(t-\tau) \\
v_{2}(t-\tau)
\end{array}\right] u(t-\tau) .
\end{aligned}
$$

Next, (6.20) can be written in the form

$$
\begin{aligned}
\dot{x}_{1}(t)= & a_{11} x_{1}(t)+a_{12} x_{2}(t)+b_{11} v_{1}(t)+b_{12} v_{2}(t) \\
\dot{x}_{2}(t)= & a_{21} x_{1}(t)+a_{22} x_{2}(t)+b_{21} v_{1}(t)+b_{22} v_{2}(t) \\
i_{1}(t)= & c_{11,0} x_{1}(t)+c_{12,0} x_{2}(t)+d_{11,0} v_{1}(t)+d_{12,0} v_{2}(t) \\
& +\left[c_{11,1} x_{1}(t-\tau)+c_{12,1} x_{2}(t-\tau)\right] u(t-\tau) \\
& +\left[d_{11,1} v_{1}(t-\tau)+d_{12,1} v_{2}(t-\tau)\right] u(t-\tau) \\
i_{2}(t)= & c_{21,0} x_{1}(t)+c_{22,0} x_{2}(t)+d_{21,0} v_{1}(t)+d_{22,0} v_{2}(t) \\
& +\left[c_{21,1} x_{1}(t-\tau)+c_{22,1} x_{2}(t-\tau)\right] u(t-\tau) \\
& +\left[d_{21,1} v_{1}(t-\tau)+d_{22,1} v_{2}(t-\tau)\right] u(t-\tau) .
\end{aligned}
$$

In the above equations, the port voltages and currents are represented by $v_{1}, v_{2}$ and $i_{1}, i_{2}$ respectively. An equivalent network representing (6.21) - (6.24) can be constructed as shown in Fig. 6.2. Each state in the macromodel requires a separate 
node in the circuit, in this case these are nodes $n_{1}$ and $n_{2}$. The state variables, $x_{1}$ and $x_{2}$ are represented by capacitor voltages. The capacitors, $C_{n 1}$ and $C_{n 2}$ have corresponding voltages $v_{n 1}$ and $v_{n 2}$ respectively. The terms $a_{12} x_{2}, b_{11} v_{1}, b_{12} v_{2}, a_{21} x_{1}$, $b_{21} v_{1}, b_{22} v_{2}, c_{11,0} x_{1}, c_{12,0} x_{2}, d_{12,0} v_{2}, c_{21,0} x_{1}, c_{22,0} x_{2}$, and $d_{21,0} v_{1}$ can be represented by voltage controlled current sources, while the terms $a_{11} x_{1}, a_{22} x_{2}, d_{11,0} v_{1}$, and $d_{22,0} v_{2}$ can be represented by resistors. The remaining terms can be represented by delayed voltage controlled current sources. The output formulations (6.23) and (6.24) are realized as equivalent circuits in Fig. 6.2(a) and Fig. 6.2(b), respectively. Fig. 6.2(c) and Fig. 6.2(d) represent the conversion of (6.21) and (6.22), respectively, to equivalent circuits. It is straightforward to generalize the previous discussion to networks with more states and ports. In addition, the above discussion can also be modified for networks described by scattering parameters [42].

\subsection{Circuit Interface for Scattering Parameter based Macromodels}

Tabulated data is often given in the form of scattering parameters, and it was found that direct construction of an equivalent set of subcircuits from scattering parameters can lead to a decrease in computational cost while maintaining the accuracy of the model [42]. The scattering parameters of a circuit relate the incident waves to the reflected waves. Let $\left\{\tilde{a}_{i}\right\}$ and $\left\{\tilde{b}_{i}\right\}$ be the time-domain representations for the incident and reflected waves, respectively, and let $\left\{\mathfrak{L}\left(\tilde{a}_{i}\right)\right\}$ and $\left\{\mathfrak{L}\left(\tilde{b}_{i}\right)\right\}$ be the Laplace-domain representations of $\left\{\tilde{a}_{i}\right\}$ and $\left\{\tilde{b}_{i}\right\}$, respectively. Then, the scattering parameters for 


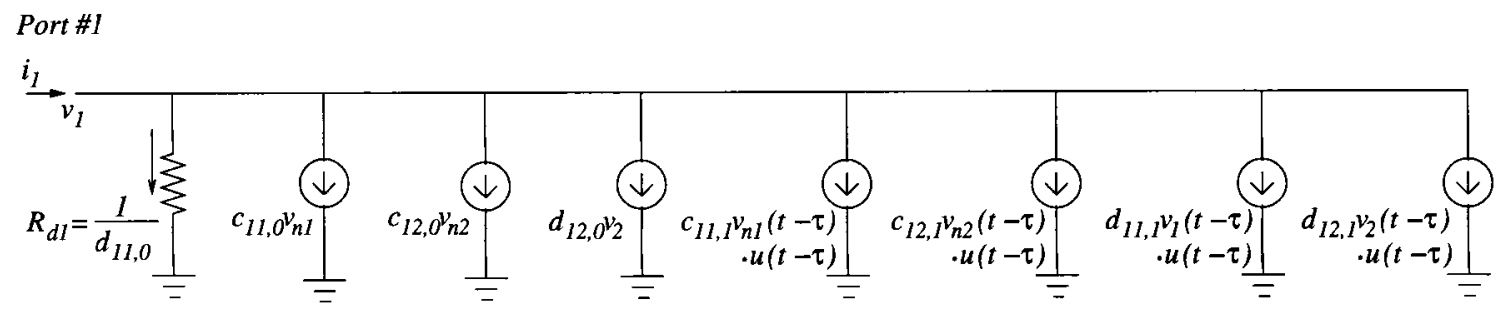

(a) Realization of (6.23)

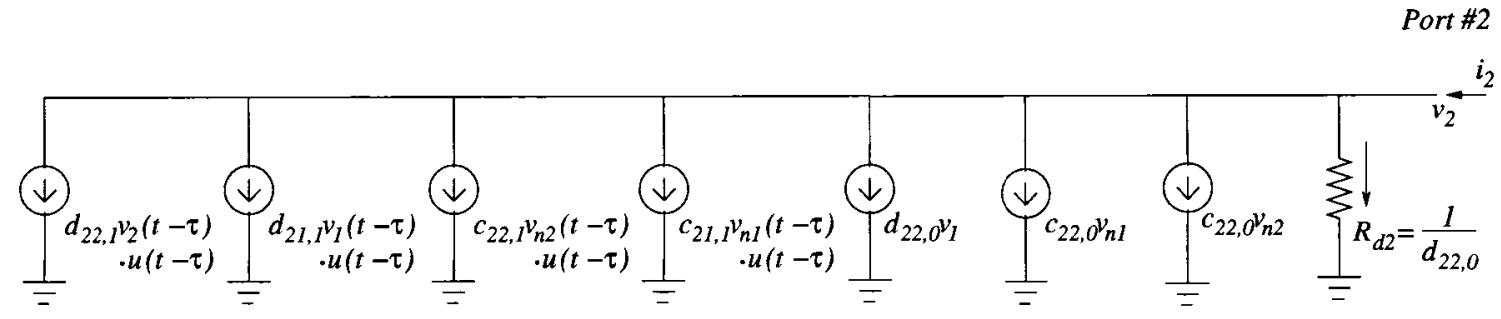

(b) Realization of (6.24)

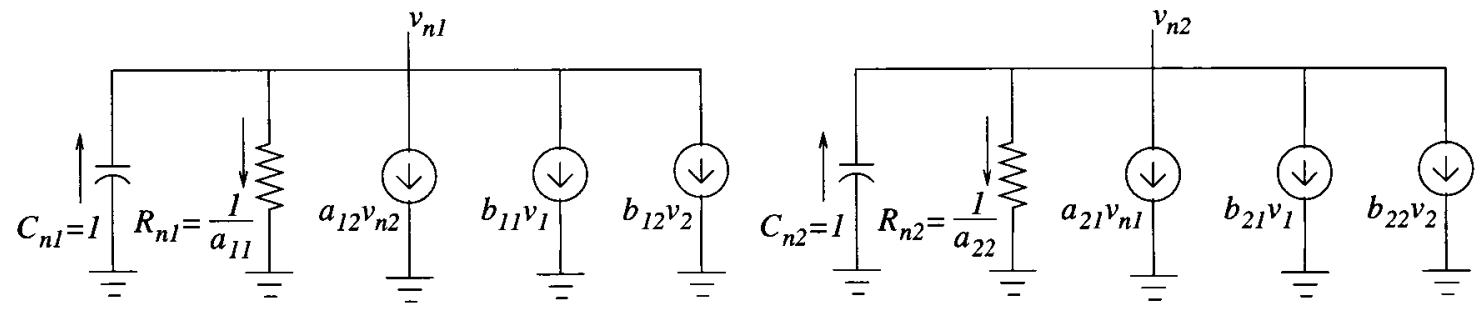

(c) Realization of (6.21)

(d) Realization of (6.22)

Fig. 6.2: Equivalent subcircuits for admittance parameters represented by DDEs (6.21)-(6.24).

the $\pi$ network in Fig. 6.1 are of the form

$$
\left[\begin{array}{cccc}
S_{11} & S_{12} & \ldots & S_{1 n_{\pi}} \\
\vdots & \vdots & \ddots & \vdots \\
S_{n_{\pi} 1} & S_{n_{\pi} 2} & \ldots & S_{n_{\pi} n_{\pi}}
\end{array}\right]\left[\begin{array}{c}
\mathfrak{L}\left(\tilde{a}_{1}\right) \\
\vdots \\
\mathfrak{L}\left(\tilde{a}_{n_{\pi}}\right)
\end{array}\right]=\left[\begin{array}{c}
\mathfrak{L}\left(\tilde{b}_{1}\right) \\
\vdots \\
\mathfrak{L}\left(\tilde{b}_{n_{\pi}}\right)
\end{array}\right]
$$

If each of the entries $S_{j k}$ in (6.25) is formulated as a delayed rational function in the form of (6.2), then macromodel synthesis can be performed on this network to obtain a system of the form of (6.3). However, in this case the inputs, $\phi(t)$, are the incident 
waves instead of the port voltages, and outputs, $\boldsymbol{y}(t)$, are the reflected waves instead of the port currents. Then, an equivalent subcircuit can be constructed using the methods of section 6.2 using the modified inputs and outputs.

In order to build a proper macromodel of the $\pi$ network, the voltages and currents must be related to the incident and reflected waves. Let $Z_{i}$ be the characteristic impedance of the $i^{\text {th }}$ port and let $\mathbf{Z}$ be a diagonal matrix such that $Z(i, i)=Z_{i}$. Then, the currents and voltages are related to the incident and reflected waves by [19]

$$
\left[\begin{array}{c}
\tilde{a} \\
\tilde{b}
\end{array}\right]=\frac{1}{2}\left[\begin{array}{cc}
\mathbf{Z}^{-1 / 2} & \mathbf{Z}^{1 / 2} \\
\mathbf{Z}^{-1 / 2} & -\mathbf{Z}^{1 / 2}
\end{array}\right]\left[\begin{array}{c}
v \\
i
\end{array}\right],
$$

or

$$
\left[\begin{array}{c}
\tilde{b} \\
i
\end{array}\right]=\left[\begin{array}{cc}
-\mathrm{I} & \mathrm{Z}^{-1 / 2} \\
2 \mathrm{Z}^{-1 / 2} & -\mathrm{Z}^{-1}
\end{array}\right]\left[\begin{array}{c}
\tilde{a} \\
v
\end{array}\right] .
$$

An equivalent subcircuit is built for (6.27), which adds two small subcircuits for each port.

\subsubsection{Example: Interface for Scattering Parameter based Macromodels}

In order to illustrate the construction of a scattering parameter subcircuit, consider a two-port example with two states and two delays at $\tau=0$ and $\tau_{1}=\tau$. The incident and reflective waves are related by (6.21) - (6.24) with $\left\{v_{j}\right\}$ and $\left\{i_{j}\right\}$ replaced by $\left\{a_{j}\right\}$ and $\left\{b_{j}\right\}$, respectively. Therefore, they are macromodeled as in Fig. 6.2. Next, these 
circuits are related to the voltage and currents by

$$
\begin{aligned}
& \tilde{b}_{1}=-\tilde{a}_{1}+Z_{1}^{-1 / 2} v_{1}, \\
& \tilde{b}_{2}=-\tilde{a}_{2}+Z_{2}^{-1 / 2} v_{2}, \\
& i_{1}=2 Z_{1}^{-1 / 2} \tilde{a}_{1}-Z_{1}^{-1} v_{1}, \\
& i_{2}=2 Z_{2}^{-1 / 2} \tilde{a}_{2}-Z_{2}^{-1} v_{2} .
\end{aligned}
$$

Equations (6.28) - (6.31) are converted into the subcircuits in Figs. 6.3(a)- 6.3(d), respectively, using the methods outlined in [3], [42].

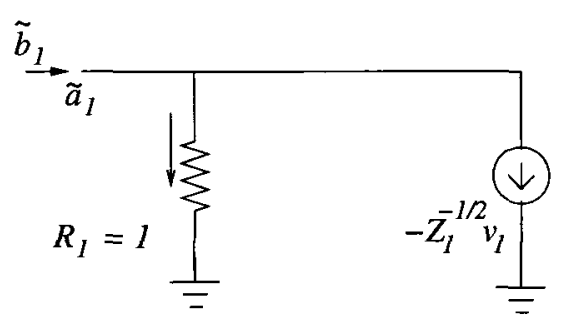

(a) Realization of (6.28)

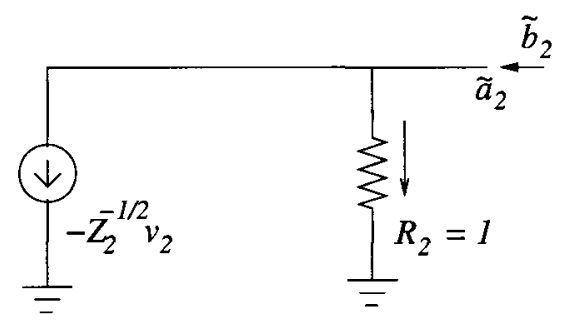

(b) Realization of (6.29)

Port \#1

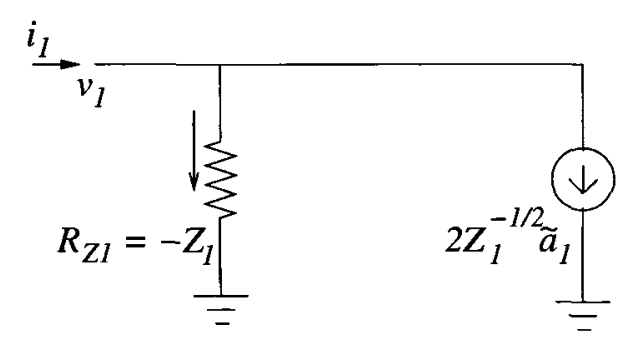

(c) Realization of (6.30)
Port \#2

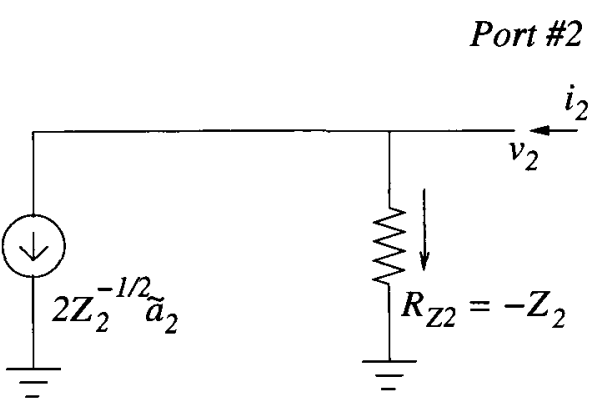

(d) Realization of (6.31)

Fig. 6.3: Equivalent subcircuits for scattering parameters. 


\subsection{Summary}

In this chapter, macromodeling techniques were developed for systems represented by delayed rational functions. The matrix transfer function of delayed rational functions is first converted into a delayed state-space formulation, equivalent to a set of DDEs. Next, a set of equivalent subcircuits is constructed to represent the DDEs in SPICElike simulators. These techniques are applied to the examples in chapters 4 and 5 . 


\section{Chapter 7}

\section{Conclusions and Future Work}

This chapter contains a summary of the work presented in this thesis. In addition, the direction of future work is also discussed.

\subsection{Summary}

In this thesis, several new algorithms were presented to address the important issue of macromodeling via delay extraction for networks with long-delays characterized by tabulated data. The first algorithm extends the DEPACT macromodeling algorithm for transmission lines characterized by $\mathrm{Y}, \mathrm{Z}, \mathrm{S}$, or $\mathrm{H}$ parameters. The second algorithm partitions the time-domain impulse response using wavelet decomposition. Each partition is fit with a sum of delayed exponentials using Prony's algorithm, resulting in a delayed rational function approximation. The third algorithm also constructs a delayed rational function approximation. However, this algorithm partitions the time-domain response data, $\{y(t)\}$ from an input excitation, $\{x(t)\}$, (not the im- 
pulse response) using wavelet decomposition. Next, each of the partitions is fit using TD-VF. The major advantages of the new methods are as follows.

1. The extension of the DEPACT macromodeling algorithm to tabulated $\mathrm{S}, \mathrm{Y}, \mathrm{Z}$, or $\mathrm{H}$ parameters tackles the shortcomings of previous techniques since it employs an advanced delay extraction mechanism prior to performing rational function approximations. This leads to significantly smaller and faster macromodels for transient simulation compared to macromodels based on conventional rational function approximations.

2. The DEPACT macromodel is passive by construction, and in turn, its extension, developed in this work, is also passive by construction.

3. The delayed rational function approximation algorithms based on Prony's algorithm and the conventional TD-VF algorithm can be applied to tabulated data characterizing general networks, not just transmission lines.

4. The delayed rational function approximation algorithm based on the TD-VF algorithm can be applied directly to measured response data representing complex long delay networks.

5. Macromodels based on delayed rational function formulations are significantly smaller and simulate more efficiently in SPICE-like simulators than macromodels based on rational approximations. 


\subsection{Future Work}

The research presented in this thesis has created several possibilities for future research projects. These possibilities are described below.

1. Passivity Verification Algorithms: Although a significant reduction in size is obtained using delayed rational functions over rational functions to approximate the matrix-transfer function, there is no efficient method to verify if passivity violations exist in the delayed rational system.

2. Passivity Enforcement and Compensation Algorithms: In many cases, non-passive data, or non-passive matrix-transfer function formulations are obtained, and it is necessary to develop methods to compensate for these violations in the macromodels defined by delayed rational function approximations.

3. Determination of Common Pole Sets: The algorithms developed in this thesis did not find common pole sets for all the matrix elements. It was found that when common pole sets were used in the case of rational approximations, a significant reduction in computational cost was obtained [38]. It is expected that a similar result will hold for the delayed rational function formulations.

4. Reduction Algorithms: Although the techniques presented in this thesis reduce the size of the macromodels for devices characterized by tabulated data, model order reduction techniques may be developed to further improve the computational efficiency of these macromodels. 


\section{Appendix A}

\section{Wavelets}

The Fourier transform is an extremely useful tool for analyzing and approximating signals in both the time-domain and frequency-domain. However, one of its significant shortcomings is its inability to perform time-frequency localization. In order to overcome this difficultly, windowed Fourier transform methods were developed. These methods require a very narrow window to capture high resolution in the time-domain, which can result in closely dependent basis functions and an unstable transform. This led to the development of wavelet theory and the wavelet transform, which has evolved significantly over the past few decades into accepted time-frequency analysis tools with much more depth than Fourier analysis [48], [59], [63]. In this thesis, delay estimation via wavelet based time-frequency analysis is proposed. For this purpose, a basic review of wavelets as relevant to this thesis follows. 


\section{A.1 Orthogonal Refinement}

In order to illustrate the concept of orthogonal refinement, consider the pulse function in Fig. A.1 [48]. Fig. A.1(a) contains a coarse approximation to the function, while Fig. A.1(b) uses a refined basis to reduce the approximation error. Unfortunately, in the case of the pulse basis, all of the expansion coefficients must be recalculated to obtain the refined approximation.

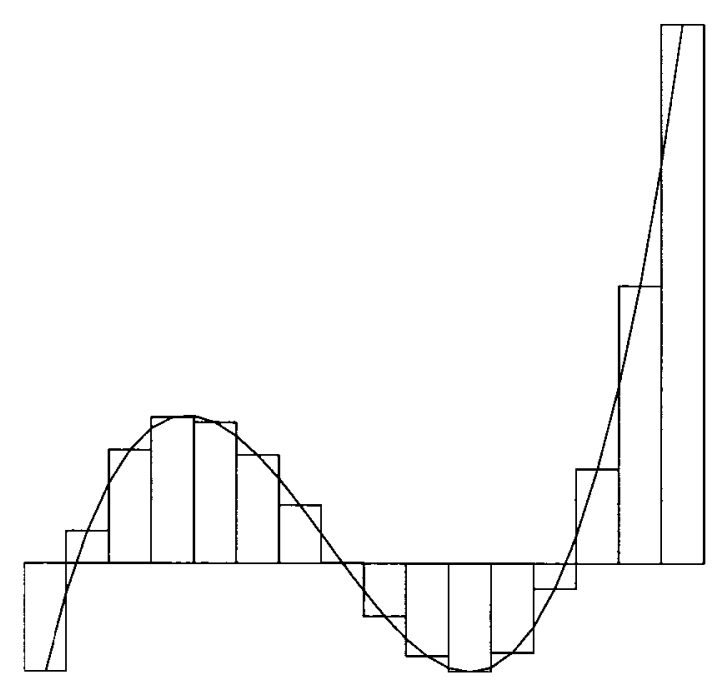

(a) Original basis.

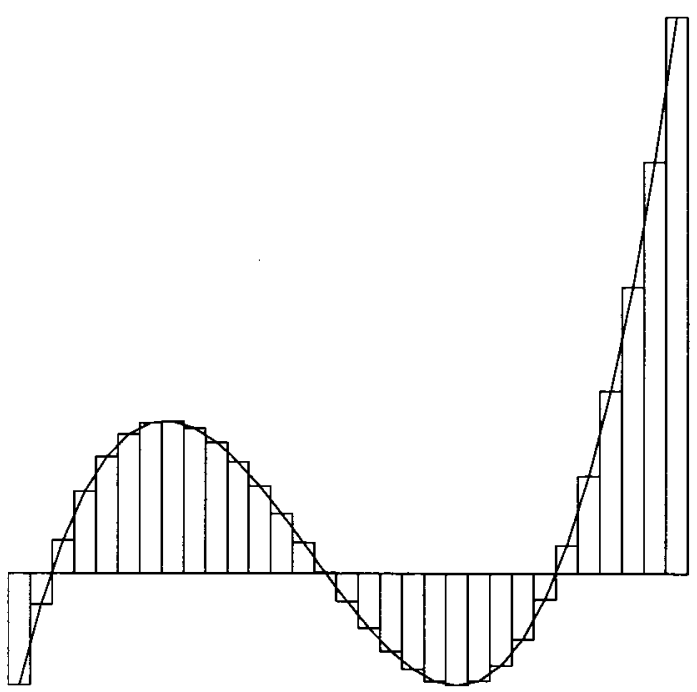

(b) Refined basis.

Fig. A.1: Refinement in the basis of pulse functions.

Define instead basis functions $\left\{\varphi_{j, k}\right\}$ for the original approximation, and let $\left\{\varphi_{j+1, k}\right\}$ be the basis set for the refined approximation. Then, a basis set that is complementary to $\left\{\varphi_{j, k}\right\}$ in the space spanned by $\left\{\varphi_{j+1, k}\right\}$ for the pulse function is easily seen to be $\left\{\psi_{j, k}\right\}$ shown in Fig. A.2 [48]. The expansion in the new refined basis set, $\left\{\varphi_{j+1, k}\right\}$, will retain half of the coefficients from the coarser basis. This set of basis functions is known as a Haar basis [59]. Basis functions $\left\{\varphi_{j, k}\right\}$ that capture average value of the approximated function are known as scaling functions, while the complementary basis 
functions $\left\{\psi_{j, k}\right\}$ that represent variation are known as wavelet functions or wavelets.

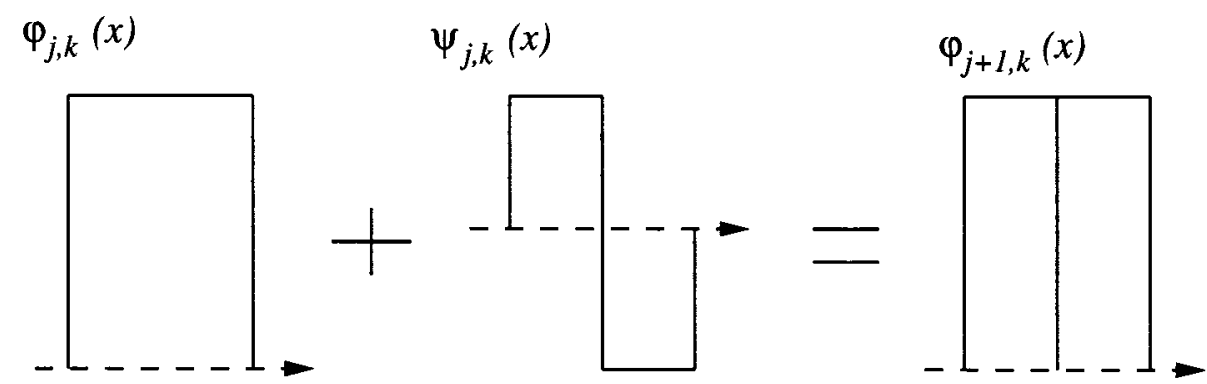

Fig. A.2: Refinement of the Haar basis using the orthogonal complement.

\section{A.2 Refinement Relations}

An important concept in wavelet analysis is the set of refinement relations that allow for a set of basis functions at level $k$ to be described in terms of scaling functions at level $k+1$. To illustrate this concept, consider the Haar basis shown in Fig. A.3 [48] for which these relations can be found by inspection.

For the general case of orthogonal wavelet basis, the refinement relations are written as

$$
\begin{aligned}
\varphi(x) & =\sqrt{2} \sum_{j=-M}^{M} h_{j} \varphi(2 x-j) \\
\psi(x) & =\sqrt{2} \sum_{j=-M}^{M} g_{j} \varphi(2 x-j) .
\end{aligned}
$$

The refinement coefficients $h_{j}$ and $g_{j}$ are not independent for orthogonal wavelets, but rather they must satisfy the symmetry relation

$$
g_{i+1}=(-1)^{i+1} h_{-i}
$$




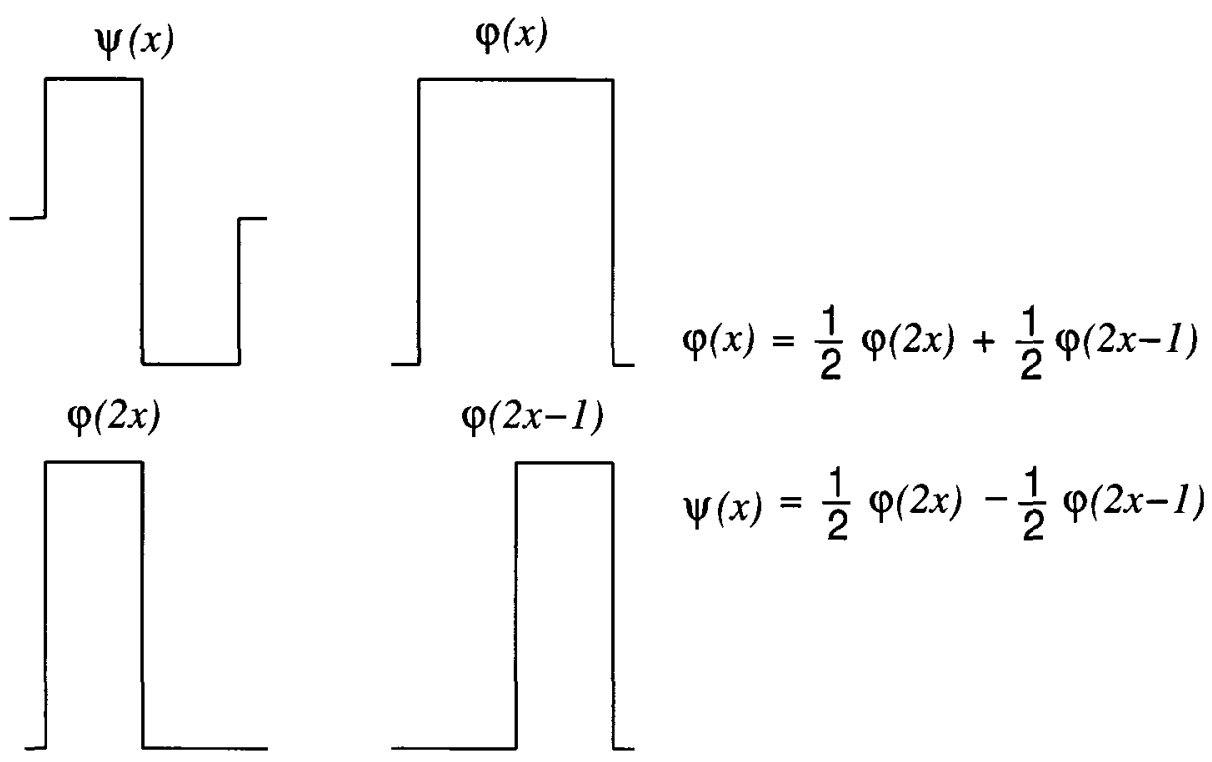

Fig. A.3: Refinement relations for the Haar basis.

and the orthogonality relations

$$
\begin{aligned}
& \sum_{l} h_{l-2 i} h_{l-2 j}=\delta_{i, j} \\
& \sum_{l} g_{l-2 i} g_{l-2 j}=\delta_{i, j} \\
& \sum_{l} h_{l-2 i} g_{l-2 j}=0 .
\end{aligned}
$$

Equations (A.1) - (A.3) together form a system of equations for the set of coefficients $\left\{h_{i}\right\}$. Each solution uniquely determines a family of orthogonal wavelets $\varphi_{j, k}(x)$ and $\psi_{j, k}(x)$, where the number of solutions is dependent on $M$, the number of nonzero coefficients $\left\{h_{i}\right\}$. If $M=4$, then the solution to this system is

$$
h=\left[\frac{1+\sqrt{3}}{4}, \frac{3+\sqrt{3}}{4}, \frac{3-\sqrt{3}}{4}, \frac{1-\sqrt{3}}{4}\right],
$$


which is the orthogonal Daubechies wavelet with four filter coefficients. This scaling function is shown in Fig. A.4 [48].

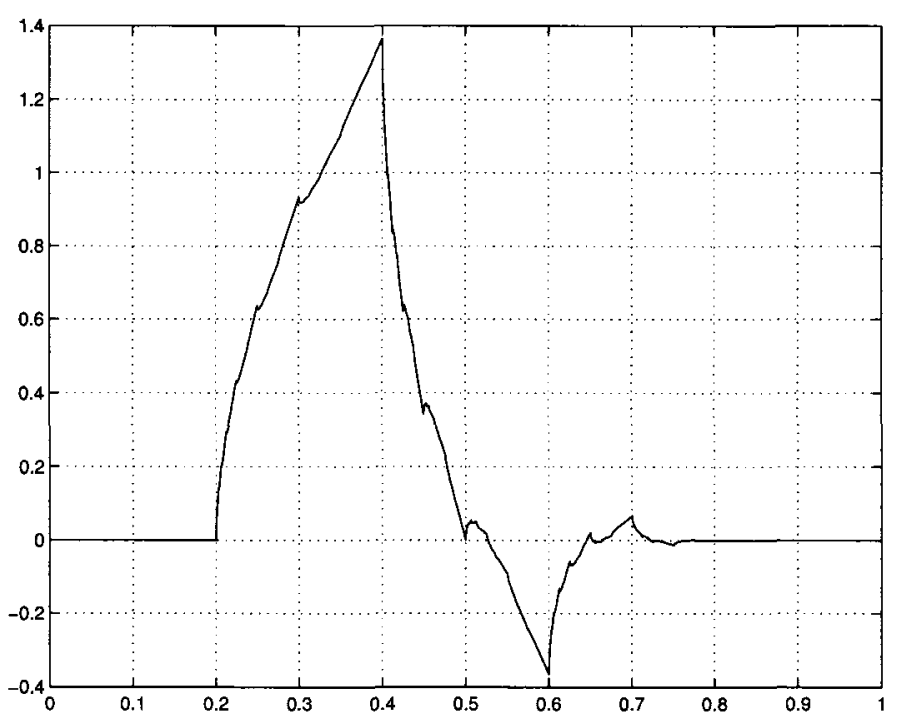

Fig. A.4: Scaling function for orthogonal Daubechies wavelet with four filter coefficients.

\section{A.3 Fast Wavelet Transform}

Analogous to the FFT, a fast wavelet transform (FWT) has been developed for the calculation of orthogonal wavelet basis expansion coefficients. In general, the transformation of a vector, $\boldsymbol{x}$, of length $N$ to $\boldsymbol{\alpha}$ of the same dimension can be accomplished using an $N \times N$ transformation matrix $\mathrm{T}$ such that

$$
\alpha=\mathbf{T} x .
$$

In order to illustrate the FWT, consider the Haar basis with one level of each of the scaling function and wavelet. Computing approximation coefficients corresponding to 
the scaling functions, $\varphi_{i}(\zeta)=\varphi(\zeta-i)$, requires computing a moving average of the vector $\boldsymbol{x}$ over two samples. This is equivalent to convolving $\boldsymbol{x}$ with a finite impulse response (FIR) digital filter with filter coefficients equal to $h=\left[\frac{1}{2}, \frac{1}{2}\right]$ [63]. Similarly, wavelet coefficients can be computed by convolving $\boldsymbol{x}$ with the FIR described by the coefficients $g=\left[-\frac{1}{2}, \frac{1}{2}\right]$.

If $\boldsymbol{x}$ contains $k$ samples, then the low-pass filter $H(z)$ in Fig. A.5 [48] will produce a vector of $k$ approximation coefficients, $\boldsymbol{a}$. The high-pass filter, $G(z)$, will produce a vector of $k$ detail coefficients, $\boldsymbol{d}$. This is twice the amount of information that was originally input to the system, therefore, the process of downsampling is performed whereby the sampling frequency of $\boldsymbol{a}$ and $\boldsymbol{d}$ is halved. The result is the two coefficient vectors, $\boldsymbol{a}$ and $\boldsymbol{d}$, combine to form the vector $\boldsymbol{\alpha}$ in (A.8).

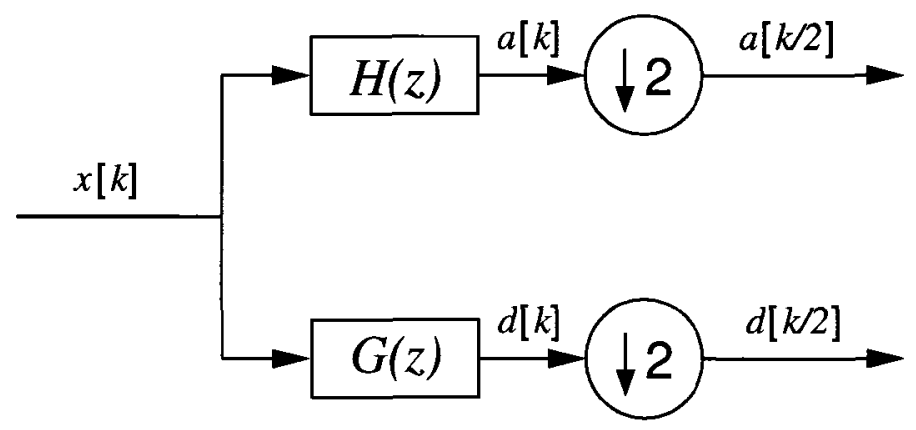

Fig. A.5: Computing one level of FWT with FIR filter bank ( $x[k]$ represents a vector of length $k$ ).

The filter coefficients for the FWT are actually the refinement coefficients for any wavelet basis (not just Haar) that satisfies the orthogonality conditions. Let $h=\left[h_{0}, h_{1}, \ldots, h_{M-1}\right]$ and $g=\left[g_{0}, g_{1}, \ldots, g_{M-1}\right]$ be filter coefficients for an arbitrary 
orthogonal wavelet family, then (A.8) can be written [66]

$$
\alpha=\left[\begin{array}{c}
a_{1} \\
d_{1} \\
a_{2} \\
d_{2} \\
a_{3} \\
d_{3} \\
\vdots
\end{array}\right]=\left[\begin{array}{ccccccccc}
h_{0} & h_{1} & \ldots & h_{M-1} & 0 & 0 & 0 & 0 & \ldots \\
g_{0} & g_{1} & \ldots & g_{M-1} & 0 & 0 & 0 & 0 & \ldots \\
0 & 0 & h_{0} & h_{1} & \ldots & h_{M-1} & 0 & 0 & \ldots \\
0 & 0 & g_{0} & g_{1} & \ldots & g_{M-1} & 0 & 0 & \ldots \\
0 & 0 & 0 & 0 & h_{0} & h_{1} & \ldots & h_{M-1} & \ldots \\
0 & 0 & 0 & 0 & g_{0} & g_{1} & \ldots & g_{M-1} & \ldots \\
\vdots & \vdots & \vdots & \vdots & \vdots & \vdots & \vdots & \vdots & \ddots
\end{array}\right]\left[\begin{array}{c}
x_{1} \\
x_{2} \\
x_{3} \\
x_{4} \\
x_{5} \\
x_{6} \\
\vdots
\end{array}\right]
$$

where $M$ is the number of refinement coefficients and $\mathbf{T}$ is a sparse matrix with a block diagonal structure. The wavelet transform is can be performed several times in sequence, each time the FWT is applied to the approximation coefficients from the previous step. This is known as a wavelet decomposition tree, and the process is shown in Fig. A.6. It is used to obtain a multilevel expansion of the original function in terms of the wavelet basis. All the approximation coefficients contain the pertain to the low-frequency content, while the high-frequency content is contained in the detail coefficients.

\section{A.4 Boundary Wavelets}

In the former section, a general description of the FWT was given. In most cases, the general transform has the effect of producing large amplitude wavelet coefficients at the boundaries, as if the function were discontinuous. In order to overcome this, the usual filters are replaced by boundary filters at the signal boundaries. The FWT 


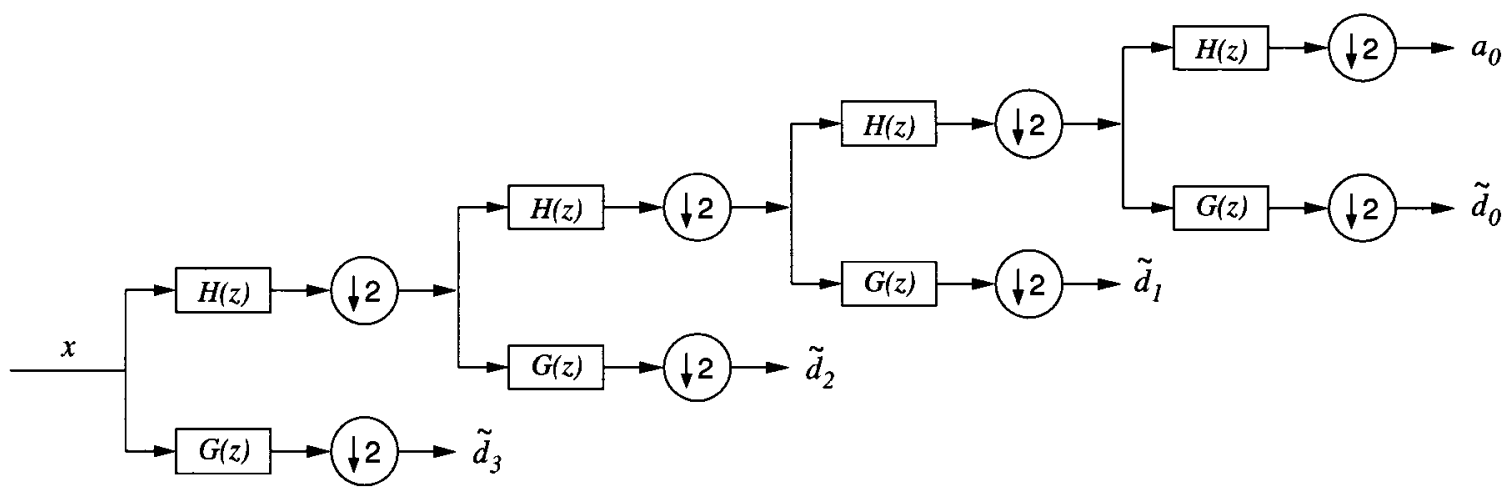

Fig. A.6: Wavelet decomposition tree $\left(\tilde{d}_{i}\right.$ represents the vector of detail coefficients from each transform).

transform relations are modified to obtain the following relations [63]. If $0 \leq k<$ $M / 2$, then

$$
\begin{aligned}
a_{k} & =\sum_{l=0}^{M / 2-1} H_{k, l}^{l e f t} x_{l}+\sum_{m=M / 2}^{M / 2-2 k} h_{k, m}^{l e f t} x_{m} \\
d_{k} & =\sum_{l=0}^{M / 2-1} G_{k, l}^{l e f t} x_{l}+\sum_{m=M / 2}^{M / 2-2 k} g_{k, m}^{l e f t} x_{m}
\end{aligned}
$$

If $M / 2<k \leq n-M / 2$, then

$$
\begin{aligned}
a_{k} & =\sum_{l=0}^{M-1} h_{l} x_{l}, \\
d_{k} & =\sum_{l=0}^{M-1} g_{l} x_{l} .
\end{aligned}
$$


Finally, if $-M / 2 \leq k<0$

$$
\begin{aligned}
a_{n+k} & =\sum_{l=-M / 2}^{-1} H_{k, l}^{r i g h t} x_{n / 2+l}+\sum_{m=-M / 2+2 k+1}^{-M / 2-1} h_{k, m}^{r i g h t} x_{n / 2+m}, \\
d_{n+k} & =\sum_{l=-M / 2}^{-1} G_{k, l}^{r i g h t} x_{n / 2+l}+\sum_{m=-M / 2+2 k+1}^{-M / 2-1} g_{k, m}^{r i g h t} x_{n / 2+m} .
\end{aligned}
$$

The variables $H_{k, l}^{r i g h t}, h_{k, m}^{\text {right }}, H_{k, l}^{\text {left }}, h_{k, m}^{\text {left }}, G_{k, l}^{\text {right }}, g_{k, m}^{r i g h t}, G_{k, l}^{\text {left }}$, and $g_{k, m}^{\text {left }}$ are the left and right border coefficients [63]. $h_{l}$ and $g_{l}$ are defined in (A.9) and $n$ is the number of data points. 


\section{Bibliography}

[1] C. Paul, Analysis of multiconductor transmission lines. New York, NY: John Wiley and Sons Inc., 1994.

[2] M. Nakhla and R. Achar, Multimedia Book Series on Signal Integrity. ON: OMNIZ Global Knowledge Corporation (www.omniz.com), 2002.

[3] R. Achar and M. S. Nakhla, "Simulation of high-speed interconnects," Proc. IEEE, vol. 89, no. 5, pp. 693-728, May 2001.

[4] R. Achar, "Model-reduction techniques for high-speed interconnect analysis," Ph.D. dissertation, Carleton University, 1998.

[5] A. Deutsch, "Electrical characteristics of interconnections for high-performance systems," Proc. IEEE, vol. 86, no. 2, pp. 315-357, February 1998.

[6] R. Achar, P. K. Gunupudi, M. Nakhla, and E. Chiprout, "Passive interconnect reduction algorithm for distributed/measured networks," IEEE Trans. Circuits Syst. II, vol. 47, no. 4, pp. 287-301, April 2000. 
[7] N. M. Nakhla, A. Dounavis, R. Achar, and M. S. Nakhla, "DEPACT: Delay extraction-based passive compact transmission-line macromodeling algorithm," IEEE Trans. Adv. Packag., vol. 28, no. 1, pp. 13-23, February 2005.

[8] N. Nakhla, "Analytical algorithms for macromodeling and sensitivity analysis of high-speed interconnects," Master's thesis, Carleton University, September 2005.

[9] A. Dounavis, N. Nakhla, R. Achar, and M. Nakhla, "Delay extraction and passive macromodeling of lossy coupled transmission lines," Proceedings of the IEEE Workshop on Electrical Performance of Electronic Packaging, pp. 251-254, October 2003 .

[10] A. Dounavis, R. Achar, and M. Nakhla, "A general class of passive macromodels for lossy multiconductor transmission lines," IEEE Trans. Microw. Theory Tech., vol. 49, no. 10, pp. 1686-1696, October 2001.

[11] A. Dounavis, R. Achar, and M. S. Nakhla, "Addressing transient errors in passive macromodels of distributed transmission-line networks," IEEE Trans. Microw. Theory Tech., vol. 50, no. 12, pp. 2759-2768, December 2002.

[12] A. Dounavis, X. Li, R. Achar, and M. S. Nakhla, "Passive closed-form transmission-line model for general-purpose circuit simulators," IEEE Trans. Microw. Theory Tech., vol. 47, no. 12, pp. 2450-2459, December 1999.

[13] A. Dounavis, R. Achar, and M. S. Nakhla, "Efficient passive circuit models for distributed networks with frequency-dependent parameters," IEEE Trans. Adv. Packag., vol. 23, no. 3, pp. 382-392, August 2000. 
[14] S. Grivet-Talocia, H.-M. Huang, A. Ruehli, F. Canavero, and I. Elfadel, "Transient analysis of lossy transmission lines: an efficient approach based on the method of characteristics," IEEE Trans. Adv. Packag., vol. 27, no. 1, pp. 45-46, February 2004.

[15] F.-Y.Chang, "The generalized method of characteristics for waveform relaxation analysis of lossy coupled transmission lines," IEEE Trans. Microw. Theory Tech., vol. 37, no. 12, pp. 2028-2038, December 1989.

[16] F. Branin, "Transient analysis of lossless transmission lines," Proc. IEEE, vol. 55, no. 11, pp. 2012-2013, November 1967.

[17] D. Kuznetsov and J. Schutt-Aine, "Optimal transient simulation of transmission lines," IEEE Trans. Circuits Syst. I, vol. 43, no. 2, pp. 110-121, February 1996.

[18] C. P. Coelho, J. R. Phillips, and L. M. Silveira, "Robust rational function approximation algorithm for model generation," Proceedings of the IEEE Design Automation Conference, pp. 207-212, June 1999.

[19] — - "Passive constrained rational approximation algorithm using nevanlinnapick interpolation," Proceedings of the IEEE Design, Automation and Test in Europe, pp. 923 - 930, March 2002.

[20] W. Beyene and J. Schutt-Aine, "Efficient transient simulation of high-speed interconnects characterized by sampled data," IEEE Trans. Compon., Packag., Manuf. Technol. B, vol. 21, pp. 105-114, February 1998. 
[21] W. Beyene and J. Schutt-Aini, "Interconnect simulation using order reduction and scattering parameters," IEEE Electronic Components and Technology Conference, pp. 627-631, May 1998.

[22] W. Hendrickx and T. Dhaene, "A discussion of rational approximation of frequency domain responses by vector fitting," IEEE Trans. Power Del., vol. 21, no. 1, pp. 441-443, February 2006.

[23] R. Mandrekar and M. Swaminathan, "Delay extraction from frequency domain data for causal macro-modeling of passive networks," Proceedings of the IEEE International Symposium on Circuits and Systems, vol. 6, pp. 5758-5761, May 2005.

[24] R. Mandrekar, K. Srinivasan, E. Engin, and M. Swaminathan, "Co-simulation of signal and power delivery networks with causality," Proceedings of the IEEE Workshop on Electrical Performance of Electronic Packaging, pp. 337-340, October 2005 .

[25] R. Mandrekar and M. Swaminathan, "Causality enforcement in transient simulation of passive networks through delay extraction," Proceedings of the IEEE Workshop on Signal Propagation on Interconnects, pp. 25-28, May 2005.

[26] R. Mandrekar, K. Srinivasan, E. Engin, and M. Swaminathan, "Causality enforcement in transient co-simulation of signal and power delivery networks," IEEE Trans. Adv. Packag., vol. 30, no. 2, pp. 270-278, May 2007. 
[27] L. D. Tommasi and B. Gustavsen, "Low order transmission line modeling by modal decomposition and minimum phase shift fitting," Proceedings of the IEEE Workshop on Signal Propagation on Interconnects, pp. 89-92, May 2006.

[28] B. Gustavsen, "Time delay identification for transmission line modeling," Proceedings of the IEEE Workshop on Signal Propagation on Interconnects, pp. 103-106, May 2004.

[29] S. Grivet-Talocia, "Delay-based macromodels for long interconnects via timefrequency decompositions," Proceedings of the IEEE Workshop on Electrical Performance of Electronic Packaging, pp. 199-202, October 2006.

[30] B. Gustavsen and A. Semlyen, "Rational approximation of frequency domain responses by vector fitting," IEEE Trans. Power Del., vol. 14, no. 3, pp. 10521061, July 1999.

[31] B. Gustavsen, "Improving the pole relocating properties of vector fitting," IEEE Trans. Power Del., vol. 21, no. 3, pp. 1587 - 1592, July 2006.

[32] ——, "Relaxed vector fitting algorithm for rational approximation of frequency domain responses," Proceedings of the IEEE Workshop on Signal Propagation on Interconnects, pp. 97 - 100, May 2006.

[33] D. Deschrijver and T. Dhaene, "Rational modeling of spectral data using orthonormal vector fitting," Proceedings of the IEEE Workshop on Signal Propagation on Interconnects, pp. 111-114, May 2005.

[34] G. Antonini, D. Deschrijver, and T. Dhaene, "A comparative study of vector fitting and orthonormal vector fitting techniques for emc applications," Proceedings 
of the IEEE International Symposium on Electromagnetic Compatibility, vol. 1, pp. 6-11, August 2006.

[35] S. Grivet-Talocia and M. Bandinu, "Improving the convergence of vector fitting for equivalent circuit extraction from noisy frequency responses," IEEE Trans. Electromagn. Compat., vol. 48, no. 1, pp. 104-120, February 2006.

[36] S. Grivet-Talocia, "Package macromodeling via time-domain vector fitting," IEEE Microw. Wireless Compon. Lett., vol. 13, no. 11, pp. 472-474, November 2004 .

[37] S. Grivet-Talocia, F. G. Canavero, I. Stievano, and I. Maio, "Circuit extraction via time-domain vector fitting," Proceedings of the IEEE International Symposium on Electromagnetic Compatibility, vol. 3, pp. 1005-1010, August 2004.

[38] D. Saraswat, R. Achar, and M. S. Nakhla, "A fast algorithm and practical considerations for passive macromodeling of measured/simulated data," IEEE Trans. Adv. Packag., vol. 27, no. 1, pp. 57-70, February 2004.

[39] — - "Global passivity enforcement algorithm for macromodels of interconnect subnetworks characterized by tabulated data," IEEE Trans. VLSI Syst., vol. 13, no. 7 , pp. 819-832, July 2005.

[40] — - "Fast passivity verification and enforcement via reciprocal systems for interconnects with large order macromodels," IEEE Trans. VLSI Syst., vol. 15, no. 1, pp. 48-59, January 2007.

[41] D. Saraswat, "Global compact passive macromodeling algorithms for high-speed circuits," Ph.D. dissertation, Carleton University, 2006. 
[42] R. Neumayer, F. Haslinger, A. Stelzer, and R. Weigel, "Synthesis of spicecompatible broadband electrical models from n-port scattering parameter data," Proceedings of the IEEE International Symposium on Electromagnetic Compatibility, vol. 1, pp. 469-474, August 2002.

[43] E. Chiprout and M. S. Nakhla, "Analysis of interconnect networks using complex frequency hopping," IEEE Trans. Comput.-Aided Design Integr. Circuits Syst., vol. 14, no. 2, pp. 186-200, February 1995.

[44] A. Odabasioglu, M. Celik, and L. Pileggi, "PRIMA : Passive reduced-order interconnect macromodeling algorithm," IEEE Trans. Comput.-Aided Design Integr. Circuits Syst., vol. 17, no. 8, pp. 645-654, August 1998.

[45] A. Charest, M. Nakhla, and R. Achar, "Passive macromodeling of transmission line type interconnects characterized by tabulated data," Proceedings of the IEEE North-East Workshop on Circuits and Systems, pp. 1421-1424, August 2007.

[46] A. Charest, D. Saraswat, M. Nakhla, R. Achar, and N. Soveiko, "Compact macromodeling of high-speed circuits via delayed rational functions," accepted for publication by Microwave and Wireless Components Letters.

[47] _ _ "Compact macromodeling of high-speed circuits via delayed rational functions using time-domain vector fit," submitted to IEEE Transactions on Microwave Theory and Techniques.

[48] N. Soveiko, "Wavelets for efficient algorithms in electronic design automation," Ph.D. dissertation, Carleton University, 2003.

[49] HSPICE Signal Integrity Guide. V-2004.03, Synopsys, March 2004. 
[50] Virtuoso(c) SpectreRF Simulation Option User Guide. Product Version 5.1.41, Cadence Design Systems, November 2004.

[51] Analog Library Reference Guide. Product Version 5.1.41, Cadence Design Systems, December 2004.

[52] S-Parameter Simulation. Agilent Technologies, September 2004.

[53] P. Triverio, S. Grivet-Talocia, M. S. Nakhla, F. Canavero, and R. Achar, "Stability, causality, and passivity in electrical interconnect models," submitted to be published IEEE Transactions on Advanced Packaging 2007.

[54] D. Youla, "Physical realizability criteria," IRE Trans. Circuit Theory, vol. 7, pp. 50-68, August 1960.

[55] E. Kuh and R. Rohrer, Theory of Active Linear Networks. San Francisco, CA:Holden-Day, 1967.

[56] L. Weiss and R.N.McDonough, "Prony's method, z-transform, and padé approximation," SIAM Review, vol. 5, no. 2, pp. 145-149, April 1963.

[57] L. N. Trefethen and D. Bau, Numerical Linear Algebra. SIAM, 1997.

[58] M. E. Valkenburg, Introduction to Modern Network Synthesis. NY: John Wiley \& Sons Inc., 1960.

[59] I. Daubechies, Ten Lectures on Wavelets. San Diego:Academic Press, 1998.

[60] S. H. Cheng, N. J. Higham, C. S. Kenneys, and A. J. Laub, "Approximating the logarithm of a matrix to specified accuracy," SIAM Journal on Matrix Analysis and Applications, vol. 22, no. 4, pp. 1112-1125, 2001. 
[61] M. Grotschel, L. Lovasz, and A. Schrijver, Geometric Algorithms and Combinatorial Optimization. Springer, 1988.

[62] T. W. Parks and C. S. Burrus, Digital filter design. New York: John Wiley and Sons, 1987.

[63] S. Mallat, A Wavelet Tour of Signal Processing, 2nd ed. Academic Press, 1999.

[64] S. Goedecker, Wavelets and their application for the solution of partial differential equations in physics. Presses polytechniques et universitaires romandes, 1998.

[65] T. M. Apostol, Calculus, 2nd ed. NY: John Wiley \& Sons Inc., 1967, vol. 1.

[66] Y. Nievergelt, Wavelets Made Easy. Boston: Birkhauser, 1999. 